Supporting Information

Aqueous Compatible Protocol to Both Alkyl and Aryl Thioamide Synthesis Jianpeng Wei, ${ }^{\dagger}$ Yiming $\mathrm{Li}^{\dagger}{ }^{\dagger}$ and Xuefeng Jiang ${ }^{*}, \dagger$

'Shanghai Key Laboratory of Green Chemistry and Chemical Process, Department of Chemistry, East China Normal University, Shanghai 200062, P. R. China

xfjiang@chem.ecnu.edu.cn

\title{
Index
}

I. General Information...................................................... 2

II. Conditions Optimization for Aryl Aldehyde.......................... 3

III. Hydrolysis of the Formamides by Sodium Sulfide....................... 4

IV. The Synthetic Procedure and Data for Table $2 \ldots \ldots \ldots \ldots \ldots \ldots \ldots \ldots \ldots \ldots . . . .5$

V. The Synthetic Procedure and Data for Table 3...................... 13

VI. $\quad$ References........................................................... 29

VII. NMR Spectra........................................................30 


\section{General Information}

\section{NMR spectrum:}

${ }^{1} \mathrm{H}$ and ${ }^{13} \mathrm{C}$ NMR spectra were collected on $400 \mathrm{MHz}$ NMR spectrometers (Bruker AVANCE) using $\mathrm{CDCl}_{3}$. Chemical shifts are reported in parts per million (ppm). Chemical shifts for protons are reported in parts per million downfield and are referenced to residual protium in the NMR solvent $\left(\mathrm{CHCl}_{3}=\delta 7.26\right)$. Chemical shifts for carbon are reported in parts per million downfield and are referenced to the carbon resonances of the solvent $\left(\mathrm{CDCl}_{3}=\delta 77.0\right)$. Data are represented as follows: chemical shift, multiplicity $(\mathrm{br}=$ broad, $\mathrm{s}=$ singlet, $\mathrm{d}=$ doublet, $\mathrm{t}=$ triplet, $\mathrm{q}=$ quartet, $\mathrm{m}=$ multiplet), coupling constants in Hertz (Hz), integration.

Mass spectroscopy: Mass spectra were in general recorded on a Shimadzu GCMS-QP2010 Ultra and a HP 5989A mass selective detector.

Chromatography: Column chromatography was performed with silica gel (300-400 mesh ASTM).

IR: TENSOR (27) Series FT-IR Spectrometers 
II. Conditions Optimization for Aryl Aldehyde.

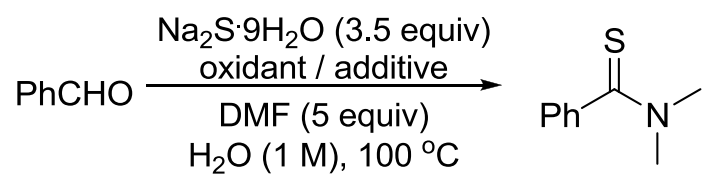

\begin{tabular}{cccc|}
\hline entry & $\begin{array}{c}\text { oxidant } \\
\text { (equiv) }\end{array}$ & $\begin{array}{c}\text { additive } \\
\text { (equiv) }\end{array}$ & $\begin{array}{c}\text { yield (\%) } \\
\text { (equ }\end{array}$ \\
1 & $\mathrm{~K}_{2} \mathrm{~S}_{2} \mathrm{O}_{8}(1.8)$ & $\mathrm{Py}(2)$ & 80 \\
2 & $\mathrm{~K}_{2} \mathrm{~S}_{2} \mathrm{O}_{8}(1.8)$ & $/$ & 80 \\
3 & $\mathrm{~K}_{2} \mathrm{~S}_{2} \mathrm{O}_{8}(2.5)$ & $/$ & 74 \\
4 & $\mathrm{TBHP}(2.5)$ & $/$ & 29 \\
5 & $\mathrm{BPO}(2.5)$ & $/$ & 86 \\
6 & $\mathrm{BPO}(2.5)$ & $/$ & trace $^{b}$ \\
7 & $/$ & $/$ & 20 \\
8 & $/$ & $/$ & $6^{c}$ \\
9 & $/$ & $/$ & $\mathrm{NR}^{d}$ \\
10 & $\mathrm{BPO}(2.5)$ & $/$ & $\mathrm{NR}^{d}$
\end{tabular}

${ }^{a}$ Isolated yields. ${ }^{b} \mathrm{Me}_{2} \mathrm{NH}$ (aq.) was used instead of DMF. ${ }^{c}$ Reaction was conducted in $\mathrm{N}_{2} \cdot{ }^{d} \mathrm{~S}_{8}$ was used instead of $\mathrm{Na}_{2} \mathrm{~S} 9 \mathrm{H}_{2} \mathrm{O}$. 
III. Hydrolysis of the Formamides by Sodium Sulfide.

\begin{tabular}{|c|c|c|}
\hline $\begin{array}{c}\mathrm{R}^{2} \\
\stackrel{1}{\mathrm{~N}} \\
\mathrm{~N}\end{array}$ & $\mathrm{Na}_{2} \mathrm{~S} \cdot 9 \mathrm{H}_{2} \mathrm{O}$ (0.7 equiv) & $R^{2} \cdot \mathrm{H}_{\mathrm{R}^{1}}^{\mathrm{H}}$ \\
\hline $\mathrm{R}^{1^{-1}}-\mathrm{CHO}$ & $\mathrm{H}_{2} \mathrm{O}, 100^{\circ} \mathrm{C}, 12 \mathrm{~h}$ & \\
\hline entry & $\mathrm{R}^{1}, \mathrm{R}^{2}$ & yield $(\%)^{a}$ \\
\hline 1 & & 91 \\
\hline 2 & $\mathrm{R}^{1}=\mathrm{H}, \mathrm{R}^{2}=\mathrm{Bn}$ & 86 \\
\hline 3 & $\mathrm{R}^{1}=\mathrm{H}, \mathrm{R}^{2}=\mathrm{PhCH}_{2} \mathrm{CH}_{2}$ & 78 \\
\hline
\end{tabular}

${ }^{a}$ Isolated yields. 
IV. The Synthetic Procedure and Data for Table 2.

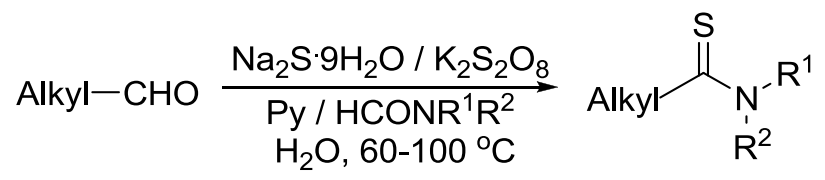

To a Schlenk tube were added alkyl aldehyde (0.3 mmol), $\mathrm{Na}_{2} \mathrm{~S} 9 \mathrm{H}_{2} \mathrm{O}(1.05$ mmol), $\mathrm{K}_{2} \mathrm{~S}_{2} \mathrm{O}_{8}(0.54 \mathrm{mmol})$, pyridine $(1.5 \mathrm{mmol}), N$-substituted formamide (1.5 mmol) and $\mathrm{H}_{2} \mathrm{O}(0.6 \mathrm{~mL})$ under air. The mixture was stirred at a certain temperature, and monitored by TLC. When the reaction was finished, the mixture was cooled to rt, quenched by $\mathrm{NH}_{4} \mathrm{Cl}$ (aq.) and extracted with ethyl acetate $(3 * 10 \mathrm{~mL})$. The combined extracts are dried over anhydrous sodium sulfate and concentrated. Purification by column chromatography on silica gel affords product thioamide.

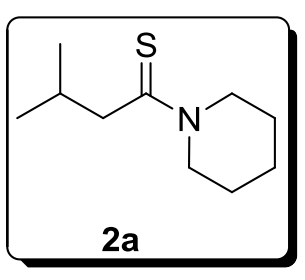

To a Schlenk tube were added 3-methylbutanal (0.3 mmol, 25.8 $\mathrm{mg}$ ), $\mathrm{Na}_{2} \mathrm{~S} 9 \mathrm{H}_{2} \mathrm{O}$ (1.05 mmol, $\left.252 \mathrm{mg}\right), \mathrm{K}_{2} \mathrm{~S}_{2} \mathrm{O}_{8}$ (0.54 mmol, $146.0 \mathrm{mg}), \quad$ pyridine $(1.5 \mathrm{mmol}, 118.5 \mathrm{mg})$, piperidine-1-carbaldehyde (1.5 mmol, $169.5 \mathrm{mg})$ and $\mathrm{H}_{2} \mathrm{O}(0.6$ $\mathrm{mL}$ ), The mixture was stirred at $100 \quad{ }^{\circ} \mathrm{C}$ for $8 \mathrm{~h}$. 3-methyl-1-(piperidin-1-yl)butane-1-thione (33.8 $\mathrm{mg}, 61 \%$ ) was obtained through column chromatography $(\mathrm{PE}: \mathrm{EA}=20: 1)$ as yellow oil, $\mathrm{R}_{\mathrm{f}}=0.55(\mathrm{PE}: \mathrm{EA}=10: 1)$. When piperidine (1.5 mmol, $127.5 \mathrm{mg}$ ) was used instead of piperidine-1-carbaldehyde in same condition, the product was not obtained through column chromatography; ${ }^{1} \mathbf{H}$ NMR (400 MHz, CDCl $\left.\mathbf{l}_{3}\right) \delta$ 4.38-4.25 (m, 2H), 3.76-3.68 (m, 2H), $2.80(\mathrm{~d}, J=7.3 \mathrm{~Hz}$ 2H), 2.24-2.07 (m, 1H), 1.78-1.60 (m, 6H), $1.02(\mathrm{~s}, 3 \mathrm{H}), 1.01(\mathrm{~s}, 3 \mathrm{H}) ;{ }^{13} \mathrm{C}$ NMR (100 MHz, $\left.\mathbf{C D C l}_{3}\right) \delta$ 201.4, 52.0, 51.6, 51.1, 28.9, 26.9, 25.4, 24.1, 22.4; IR (film) 2937, 2361, 1487, 1440, 1265, 1149, 1079, 1002, $852 \mathrm{~cm}^{-1}$; HRMS (EI) Calcd for $\mathrm{C}_{10} \mathrm{H}_{19} \mathrm{NS}$ 185.1238, Found 185.1235.

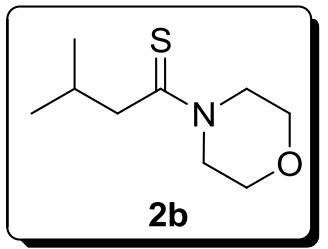

To a Schlenk tube were added 3-methylbutanal (0.3 mmol, $25.8 \mathrm{mg}), \mathrm{Na}_{2} \mathrm{~S} 9 \mathrm{H}_{2} \mathrm{O}(1.05 \mathrm{mmol}, 252 \mathrm{mg}), \mathrm{K}_{2} \mathrm{~S}_{2} \mathrm{O}_{8}(0.54$ mmol, $146.0 \mathrm{mg})$, pyridine (1.5 mmol, $118.5 \mathrm{mg})$, 
$N$-formylmorpholine $(1.5 \mathrm{mmol}, 172.5 \mathrm{mg})$ and $\mathrm{H}_{2} \mathrm{O}(0.6 \mathrm{~mL})$, The mixture was stirred at $100{ }^{\circ} \mathrm{C}$ for 9 h. 3-methyl-1-morpholinobutane-1-thione ${ }^{[4]}(32.0 \mathrm{mg}, 57 \%)$ was obtained through column chromatography $(\mathrm{PE}: \mathrm{EA}=15: 1)$ as light yellow oil, $\mathrm{R}_{\mathrm{f}}$ $=0.6(\mathrm{PE}: \mathrm{EA}=5: 1)$; ${ }^{1} \mathbf{H}$ NMR (400 MHz, $\left.\mathbf{C D C l}_{3}\right) \delta$ 4.44-4.25 (m, 2H), 3.86-3.62 $(\mathrm{m}, 6 \mathrm{H}), 2.76(\mathrm{~d}, J=7.3 \mathrm{~Hz}, 2 \mathrm{H}), 2.17-2.06(\mathrm{~m}, 1 \mathrm{H}), 0.99(\mathrm{~d}, J=6.7 \mathrm{~Hz}, 6 \mathrm{H}) ;{ }^{13} \mathbf{C}$ NMR (100 MHz, $\left.\mathbf{C D C l}_{3}\right) \delta$ 203.2, 66.6, 66.5, 51.6, 50.4, 50.1, 29.1, 22.3; IR (film) 2961, 2925, 2866, 1478, 1431, 1259, 1115, 1021, $797 \mathrm{~cm}^{-1}$; MS (EI) $\mathrm{m} / z(\%) 187$ (100), 144 (80), 86 (85).

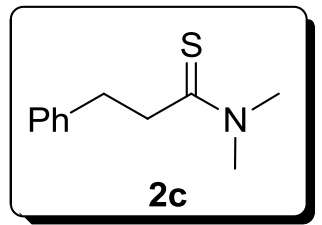

To a Schlenk tube were added 3-phenylpropanal (0.3 mmol, $40.2 \mathrm{mg}), \mathrm{Na}_{2} \mathrm{~S} \cdot 9 \mathrm{H}_{2} \mathrm{O}$ (1.05 mmol, $\left.252 \mathrm{mg}\right), \mathrm{K}_{2} \mathrm{~S}_{2} \mathrm{O}_{8}(0.54 \mathrm{mmol}$, $146.0 \mathrm{mg})$, pyridine $(1.5 \mathrm{mmol}, 118.5 \mathrm{mg})$, DMF (1.5 mmol, $109.5 \mathrm{mg})$ and $\mathrm{H}_{2} \mathrm{O}(0.6 \mathrm{~mL})$, The mixture was stirred at $100{ }^{\circ} \mathrm{C}$ for 8 h. $\mathrm{N}, \mathrm{N}$-dimethyl-3-phenylpropanethioamide ${ }^{[2]}(48.0 \mathrm{mg}, 83 \%)$ was obtained through column chromatography $(\mathrm{PE}: \mathrm{EA}=15: 1)$ as orange oil, $\mathrm{R}_{\mathrm{f}}=0.4(\mathrm{PE}: \mathrm{EA}=5$ : 1); ${ }^{1} \mathbf{H}$ NMR (400 MHz, $\left.\mathbf{C D C l}_{3}\right) \delta$ 7.32-7.27 (m, 2H), 7.24-7.20 (m, 3H), $3.48(\mathrm{~s}, 3 \mathrm{H})$, 3.14 (s, 3H), 3.13-3.09 (m, 2H), 3.09-3.03 (m, 2H); ${ }^{13} \mathbf{C}$ NMR (100 MHz, CDCl $) \delta$ 203.1, 140.6, 128.5, 128.4, 126.4, 44.9, 44.6, 41.5, 35.7; IR (film) 3025, 2963, 1514, 1496, 1393, 1260, 1093, 1017, 798, $700 \mathrm{~cm}^{-1}$; MS (EI) $\mathrm{m} / \mathrm{z}$ (\%) 193 (100), 88 (90).

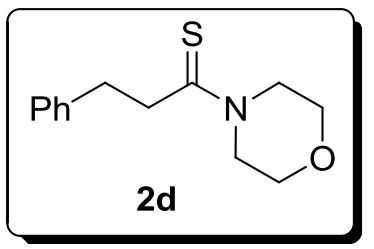

To a Schlenk tube were added 3-phenylpropanal $(0.3 \mathrm{mmol}$, $40.2 \mathrm{mg}), \mathrm{Na}_{2} \mathrm{~S} 9 \mathrm{H}_{2} \mathrm{O}(1.05 \mathrm{mmol}, 252 \mathrm{mg}), \mathrm{K}_{2} \mathrm{~S}_{2} \mathrm{O}_{8}(0.54$ mmol, $146.0 \mathrm{mg})$, pyridine (1.5 mmol, $118.5 \mathrm{mg})$, $N$-formylmorpholine (1.5 mmol, $172.5 \mathrm{mg})$ and $\mathrm{H}_{2} \mathrm{O}(0.6$ $\mathrm{mL}$ ) , The mixture was stirred at $100 \quad{ }^{\circ} \mathrm{C}$ for $3 \mathrm{~h}$. 1-morpholino-3-phenylpropane-1-thione ${ }^{[1]}(58.5 \mathrm{mg}, 83 \%)$ was obtained through column chromatography $(\mathrm{PE}: \mathrm{EA}=15: 1)$ as orange oil, $\mathrm{R}_{\mathrm{f}}=0.4(\mathrm{PE}: \mathrm{EA}=5: 1) ;{ }^{1} \mathbf{H}$ NMR (400 MHz, CDCl $)_{3} \delta$ 7.30-7.27 (m, 2H), 7.23-7.20 (m, 3H), 4.32-4.30 (m, 2H), 3.70-3.68 (m, 2H), 3.54-3.51 (m, 2H), 3.39-3.36 (m, 2H), 3.12-3.10 (m, 4H); ${ }^{13} \mathrm{C}$ NMR (100 MHz, $\left.\mathbf{C D C l}_{3}\right) \delta 202.6,140.1,128.5,128.5,126.5,66.3,66.0,50.0,49.8$, 
44.1, 35.7; IR (film) 3302, 3060, 3028, 2962, 2862, 1531, 1488, 1453, 1384, 1261, 1093, 1027, 943, 802, 767, $695 \mathrm{~cm}^{-1}$; MS (EI) m/z (\%) 235 (45), 144 (100), 91 (60).

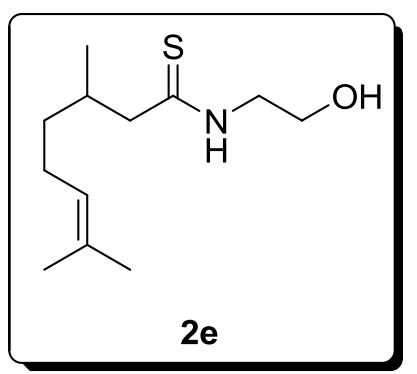

To a Schlenk tube were added citronellal $(0.3 \mathrm{mmol}, 46.2$ $\mathrm{mg}), \mathrm{Na}_{2} \mathrm{~S} 9 \mathrm{H}_{2} \mathrm{O}(1.05 \mathrm{mmol}, 252 \mathrm{mg}), \mathrm{K}_{2} \mathrm{~S}_{2} \mathrm{O}_{8}(0.54 \mathrm{mmol}$, $146.0 \mathrm{mg})$, pyridine (1.5 mmol, $118.5 \mathrm{mg})$, $N$-(2-hydroxyethyl)formamide $(1.5 \mathrm{mmol}, 133.5 \mathrm{mg})$ and $\mathrm{H}_{2} \mathrm{O}(0.6 \mathrm{~mL})$, The mixture was stirred at $100{ }^{\circ} \mathrm{C}$ for $5 \mathrm{~h}$. $N$-(2-hydroxyethyl)-3,7-dimethyloct-6-enethioamide (34.4 mg, 50\%) was obtained through column chromatography $(\mathrm{PE}: \mathrm{EA}=3: 1)$ as yellow solid, $\mathrm{R}_{\mathrm{f}}=0.50(\mathrm{PE}: \mathrm{EA}=$ 1: 1). When 2-aminoethanol (1.5 mmol, $91.5 \mathrm{mg})$ was used instead of $N$-(2-hydroxyethyl)formamide in same condition, the product was not obtained through column chromatography; ${ }^{1} \mathbf{H}$ NMR (400 $\left.\mathbf{M H z}, \mathbf{C D C l}_{3}\right) \delta 7.72$ (br, 1H), 5.10-5.07 (m, 1H), 3.94-3.83 (m, 4H), $2.70(\mathrm{dd}, J=13.2,6.1 \mathrm{~Hz}, 1 \mathrm{H}), 2.41(\mathrm{dd}, J=$ 13.1, 8.5 Hz, 1H), 2.20-2.12 (m, 1H), 2.08-1.94 (m, 2H), 1.67 (s, 3H), 1.59 (s, 3H), 1.27-1.14 (m, 2H), $0.92(\mathrm{~d}, J=6.6 \mathrm{~Hz}, 3 \mathrm{H}) ;{ }^{13} \mathbf{C}$ NMR (100 MHz, $\left.\mathbf{C D C l}_{3}\right) \delta 205.7$, 131.6, 124.2, 60.6, 54.9, 47.8, 36.5, 33.5, 25.7, 25.4, 18.9, 17.7; IR (film) 3266, 2957, 2926, 1534, 1457, 1404, 1318, 1052, $718 \mathrm{~cm}^{-1}$; HRMS (EI) Calcd for $\mathrm{C}_{12} \mathrm{H}_{23} \mathrm{NOS}$ 229.1500, Found 229.1501.

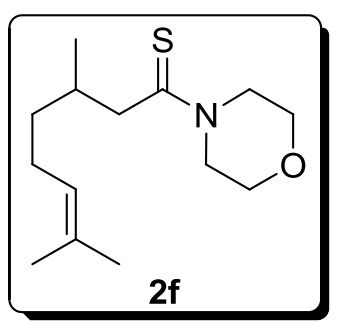

To a Schlenk tube were added citronellal (0.3 mmol, $46.2 \mathrm{mg})$, $\mathrm{Na}_{2} \mathrm{~S} 9 \mathrm{H}_{2} \mathrm{O}(1.05 \mathrm{mmol}, 252 \mathrm{mg}), \mathrm{K}_{2} \mathrm{~S}_{2} \mathrm{O}_{8}(0.54 \mathrm{mmol}, 146.0$ $\mathrm{mg})$, pyridine (1.5 mmol, $118.5 \mathrm{mg}), \mathrm{N}$-formylmorpholine (1.5 mmol, $172.5 \mathrm{mg})$ and $\mathrm{H}_{2} \mathrm{O}(0.6 \mathrm{~mL})$, The mixture was stirred $\begin{array}{llllll}\text { at } & 100 & { }^{\circ} \mathrm{C} & \text { for } & 10 & \mathrm{~h} \text {. }\end{array}$ 3,7-dimethyl-1-morpholinooct-6-ene-1-thione (46.7 mg, $61 \%$ ) was obtained through column chromatography $(\mathrm{PE}: \mathrm{EA}=15: 1)$ as light yellow oil, $\mathrm{R}_{\mathrm{f}}=0.4(\mathrm{PE}: \mathrm{EA}=5: 1)$; ${ }^{1}$ H NMR (400 MHz, CDCl $) \delta 5.06(\mathrm{t}, J=7.0 \mathrm{~Hz}, 1 \mathrm{H}), 4.36-4.34(\mathrm{~m}, 2 \mathrm{H}), 3.86-3.65$ (m, 6H), $2.86(\mathrm{dd}, J=13.4,6.2 \mathrm{~Hz}, 1 \mathrm{H}), 2.73(\mathrm{dd}, J=13.4,8.5 \mathrm{~Hz}, 1 \mathrm{H}), 2.09-1.92(\mathrm{~m}$, $3 \mathrm{H}), 1.66(\mathrm{~s}, 3 \mathrm{H}), 1.59(\mathrm{~s}, 3 \mathrm{H}), 1.51-1.39(\mathrm{~m}, 1 \mathrm{H}), 1.34-1.25(\mathrm{~m}, 1 \mathrm{H}), 0.98(\mathrm{~d}, J=6.6$ 
$\mathrm{Hz}, 3 \mathrm{H}) ;{ }^{13} \mathbf{C}$ NMR (100 MHz, $\left.\mathbf{C D C l}_{3}\right) \delta$ 203.1, 131.7, 124.2, 66.6, 66.5, 50.4, 50.2, 50.1, 36.7, 33.1, 25.7, 25.3, 19.2, 17.7; IR (film) 2963, 2906, 1477, 1432, 1259, 1084 , 1012, $792.702 \mathrm{~cm}^{-1}$; HRMS (EI) Calcd for $\mathrm{C}_{14} \mathrm{H}_{25} \mathrm{NOS} 255.1657$, Found 255.1655.

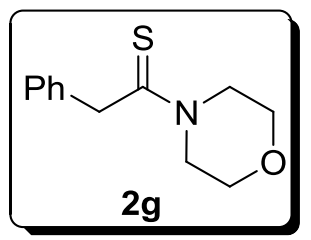

To a Schlenk tube were added 2-phenylacetaldehyde (0.3 mmol, $36.0 \mathrm{mg}$ ), $\mathrm{Na}_{2} \mathrm{~S} 9 \mathrm{H}_{2} \mathrm{O}$ (1.05 mmol, $\left.252 \mathrm{mg}\right), \mathrm{K}_{2} \mathrm{~S}_{2} \mathrm{O}_{8}(0.54 \mathrm{mmol}$, $146.0 \mathrm{mg}$ ), pyridine (1.5 mmol, $118.5 \mathrm{mg}), \mathrm{N}$-formylmorpholine (1.5 mmol, $172.5 \mathrm{mg})$ and $\mathrm{H}_{2} \mathrm{O}(0.6 \mathrm{~mL})$, The mixture was stirred at $100{ }^{\circ} \mathrm{C}$ for 9 h. 1-morpholino-2-phenylethanethione ${ }^{[3]}(30.6 \mathrm{mg}, 46 \%)$ was obtained through column chromatography $(\mathrm{PE}: \mathrm{EA}=15: 1)$ as yellow solid, $\mathrm{R}_{\mathrm{f}}=0.35$ (PE: EA = $5:$ 1); ${ }^{1} \mathbf{H}$ NMR (400 MHz, $\left.\mathbf{C D C l}_{3}\right) \delta$ 7.31-7.17 (m, 5H), 4.33-4.27 (m, 4H), 3.71-3.66 (m, 2H), 3.60-3.54 (m, 2H), 3.36-3.30 (m, 2H); ${ }^{13}$ C NMR (100 MHz, $\left.\mathbf{C D C l}_{3}\right) \delta 200.0,135.7,128.9,127.7,127.1,66.3,66.1,50.7,50.6,50.1$; IR (film) 3407, 3059, 3026, 2964, 2855, 1602, 1487, 1433, 1296, 1254, 1109, 1030, 959, 751, $705 \mathrm{~cm}^{-1}$; MS (EI) $\mathrm{m} / z$ (\%) 221 (70), 130 (75), 91 (100).

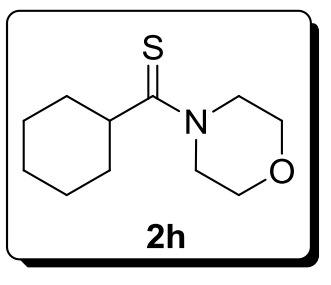

To a Schlenk tube were added cyclohexanecarbaldehyde (0.3 mmol, $33.7 \mathrm{mg}), \mathrm{Na}_{2} \mathrm{~S} 9 \mathrm{H}_{2} \mathrm{O}(1.05 \mathrm{mmol}, 252 \mathrm{mg})$, $\mathrm{K}_{2} \mathrm{~S}_{2} \mathrm{O}_{8}(0.54 \mathrm{mmol}, 146.0 \mathrm{mg})$, pyridine (1.5 mmol, $\left.118.5 \mathrm{mg}\right)$, $N$-formylmorpholine (1.5 mmol, $172.5 \mathrm{mg})$ and $\mathrm{H}_{2} \mathrm{O}(0.6 \mathrm{~mL})$,

The mixture was stirred at $100{ }^{\circ} \mathrm{C}$ for $4 \mathrm{~h}$. cyclohexyl(morpholino)methanethione ${ }^{[5]}$ (32.6 mg, $51 \%$ ) was obtained through column chromatography (PE: EA = 20: 1) as light yellow solid, $\mathrm{R}_{\mathrm{f}}=0.6(\mathrm{PE}: \mathrm{EA}=10: 1) ;{ }^{1} \mathbf{H} \mathbf{N M R}\left(400 \mathbf{M H z}, \mathbf{C D C l}_{3}\right) \delta$ 4.45-4.30 (m, 2H), 3.92-3.64 (m, 6H), $2.69(\mathrm{t}, J=10.9 \mathrm{~Hz}, 1 \mathrm{H}), 1.83-1.74(\mathrm{~m}$, 4H),1.70-1.67 (m, 3H), 1.32-1.24 (m, 3H); ${ }^{13}$ C NMR (100 MHz, CDCl $\mathbf{~ M ~}_{3} \delta$ 209.2, 66.7, 66.6, 50.0, 49.5, 47.3, 33.2, 26.1, 25.6; IR (film) 2956, 2925, 2852, 1462, 1429, 1261, 1119, 1030, $797 \mathrm{~cm}^{-1}$; MS (EI) $\mathrm{m} / z$ (\%) 213 (80), 126 (25), 86 (100).

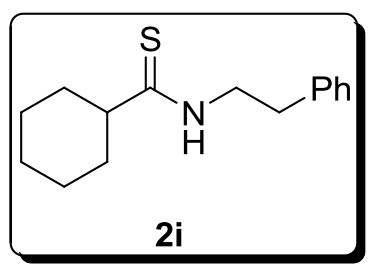

To a Schlenk tube were added cyclohexanecarbaldehyde (0.3 mmol, $33.7 \mathrm{mg}), \mathrm{Na}_{2} \mathrm{~S} 9 \mathrm{H}_{2} \mathrm{O}$ (1.05 mmol, $\left.252 \mathrm{mg}\right)$, 
$\mathrm{K}_{2} \mathrm{~S}_{2} \mathrm{O}_{8} \quad(0.54 \mathrm{mmol}, \quad 146.0 \mathrm{mg})$, pyridine $\left(\begin{array}{llll}1.5 & \mathrm{mmol}, & 118.5 \mathrm{mg}\end{array}\right)$, $N$-phenethylformamide (1.5 mmol, $223.5 \mathrm{mg})$ and $\mathrm{H}_{2} \mathrm{O}(0.6 \mathrm{~mL})$, The mixture was stirred at $100{ }^{\circ} \mathrm{C}$ for $8 \mathrm{~h}$. N-phenethylcyclohexanecarbothioamide (40.7 mg, 55\%) was obtained through column chromatography $(\mathrm{PE}: \mathrm{EA}=20: 1)$ as yellow oil, $\mathrm{R}_{\mathrm{f}}=0.50$ $(\mathrm{PE}: \mathrm{EA}=10: 1)$. When 2-phenylethanamine $(1.5 \mathrm{mmol}, 181.5 \mathrm{mg})$ was used instead of $\mathrm{N}$-phenethylformamide in same condition, the product $(9.6 \mathrm{mg}, 13 \%)$ was obtained through column chromatography; ${ }^{1} \mathbf{H}$ NMR (400 $\left.\mathbf{~ M H z , ~} \mathbf{C D C l}_{3}\right) \delta$ 7.37-7.17 (m, 5H), $7.12(\mathrm{br}, 1 \mathrm{H}), 3.95(\mathrm{dd}, J=12.5,6.8 \mathrm{~Hz}, 2 \mathrm{H}), 2.98(\mathrm{t}, J=6.9 \mathrm{~Hz}, 2 \mathrm{H}), 2.46-2.38(\mathrm{~m}$, $1 \mathrm{H}), 1.88-1.74(\mathrm{~m}, 4 \mathrm{H}), 1.69-1.66(\mathrm{~m}, 1 \mathrm{H}), 1.55-1.47(\mathrm{~m}, 2 \mathrm{H}), 1.27-1.18(\mathrm{~m}, 3 \mathrm{H}) ;{ }^{13} \mathrm{C}$ NMR (100 MHz, CDCl $) \delta$ 210.4, 138.3, 128.8, 128.7, 126.8, 55.0, 46.3, 33.7, 32.8, 25.9, 25.6; IR (film) 3243, 2928, 2853, 1524, 1450, 1280, 1131, 979, $698 \mathrm{~cm}^{-1}$; HRMS (EI) Calcd for $\mathrm{C}_{15} \mathrm{H}_{21} \mathrm{NS} 247.1395$, Found 247.1392.

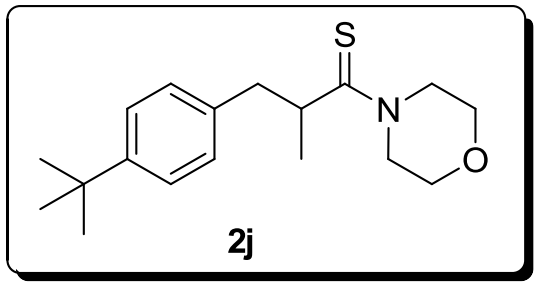

To a Schlenk tube were added lilyaldehyde $(0.3$ mmol, $61.3 \mathrm{mg}), \mathrm{Na}_{2} \mathrm{~S} 9 \mathrm{H}_{2} \mathrm{O}$ (1.05 mmol, $252 \mathrm{mg}$ ), $\mathrm{K}_{2} \mathrm{~S}_{2} \mathrm{O}_{8}$ (0.54 mmol, $\left.146.0 \mathrm{mg}\right)$, pyridine (1.5 mmol, $118.5 \mathrm{mg}), N$-formylmorpholine $(1.5 \mathrm{mmol}$, $172.5 \mathrm{mg})$ and $\mathrm{H}_{2} \mathrm{O}(0.6 \mathrm{~mL})$, The mixture was stirred at $100{ }^{\circ} \mathrm{C}$ for $20 \mathrm{~h}$. 3-(4-tert-butylphenyl)-2-methyl-1-morpholinopropane-1-thione $(59.5 \mathrm{mg}, 65 \%)$ was obtained through column chromatography $(\mathrm{PE}: \mathrm{EA}=15: 1)$ as light yellow solid, $\mathrm{R}_{\mathrm{f}}=$ 0.3 (PE: EA = $5: 1$ ); ${ }^{\mathbf{1}} \mathbf{H}$ NMR (400 MHz, $\left.\mathbf{C D C l}_{3}\right) \delta 7.28(\mathrm{~d}, J=8.3 \mathrm{~Hz}, 2 \mathrm{H}), 7.14(\mathrm{~d}$, $J=8.3 \mathrm{~Hz}, 2 \mathrm{H}), 4.82-4.74(\mathrm{~m}, 1 \mathrm{H}), 3.88-3.82(\mathrm{~m}, 1 \mathrm{H}), 3.73-3.69(\mathrm{~m}, 1 \mathrm{H}), 3.57-3.53$ (m, 1H), 3.48-3.39 (m, 3H), 3.35-3.28 (m, 1H), 3.09 (dd, $J=13.1,8.9 \mathrm{~Hz}, 1 \mathrm{H}), 2.83$ (dd, $J=13.1,5.6 \mathrm{~Hz}, 1 \mathrm{H}), 2.66-2.60(\mathrm{~m}, 1 \mathrm{H}), 1.34$ (d, $J=6.4 \mathrm{~Hz}, 3 \mathrm{H}), 1.29$ (s, 9H); ${ }^{13}$ C NMR (100 MHz, $\left.\mathbf{C D C l}_{3}\right) \delta 208.8,149.5,136.6,128.8,125.2,66.3,65.9,50.3$, 49.8, 43.8, 43.6, 34.3, 31.3, 22.2; IR (film) 3024, 2862, 1510, 1471, 1433, 1274, 1233, 1113. 1024, 983, 872, 837, $805 \mathrm{~cm}^{-1}$; HRMS (EI) Calcd for $\mathrm{C}_{18} \mathrm{H}_{27} \mathrm{NOS} 305.1813$, Found 305.1814 . 


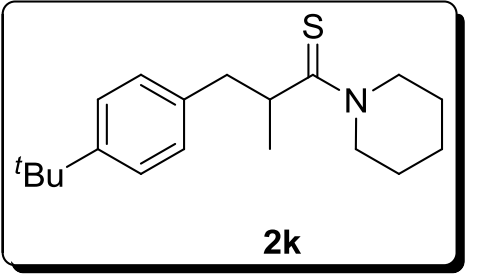

To a Schlenk tube were added lilyaldehyde $(0.3 \mathrm{mmol}$, $61.3 \mathrm{mg}), \mathrm{Na}_{2} \mathrm{~S} 9 \mathrm{H}_{2} \mathrm{O}(1.05 \mathrm{mmol}, 252 \mathrm{mg}), \mathrm{K}_{2} \mathrm{~S}_{2} \mathrm{O}_{8}$ (0.54 mmol, $146.0 \mathrm{mg})$, pyridine (1.5 mmol, $118.5 \mathrm{mg})$, piperidine-1-carbaldehyde (1.5 mmol, $169.5 \mathrm{mg})$ and $\mathrm{H}_{2} \mathrm{O} \quad(0.6 \mathrm{~mL})$, The mixture was stirred at $100{ }^{\circ} \mathrm{C}$ for $24 \mathrm{~h}$. 3-(4-tert-butylphenyl)-2-methyl-1-(piperidin-1-yl)propane-1-thione (47.3 mg, 52\%) was obtained through column chromatography $(\mathrm{PE}: \mathrm{EA}=20: 1)$ as yellow solid, $\mathrm{R}_{\mathrm{f}}=$ 0.50 (PE: EA = 10: 1). When piperidine $(1.5 \mathrm{mmol}, 127.5 \mathrm{mg})$ was used instead of piperidine-1-carbaldehyde in same condition, the product was not obtained through column chromatography; ${ }^{1} \mathbf{H}$ NMR (400 MHz, $\left.\mathbf{C D C l}_{3}\right) \delta 7.27(\mathrm{~d}, J=8.6 \mathrm{~Hz}, 2 \mathrm{H})$, $7.15(\mathrm{~d}, J=8.3 \mathrm{~Hz}, 2 \mathrm{H}), 4.70-4.59(\mathrm{~m}, 1 \mathrm{H}), 3.89-3.80(\mathrm{~m}, 1 \mathrm{H}), 3.68-3.62(\mathrm{~m}, 1 \mathrm{H})$, 3.47-3.38 (m, 1H), 3.38-3.28 (m, 1H), $3.13(\mathrm{dd}, J=13.1,7.9 \mathrm{~Hz}, 1 \mathrm{H}), 2.80(\mathrm{dd}, J=$ $13.2,6.3 \mathrm{~Hz}, 1 \mathrm{H}), 1.65-1.60(\mathrm{~m}, 1 \mathrm{H}), 1.59-1.51(\mathrm{~m}, 2 \mathrm{H}), 1.50-1.42(\mathrm{~m}, 1 \mathrm{H}), 1.45-1.35$ $(\mathrm{m}, 1 \mathrm{H}), 1.30-1.27(\mathrm{~m}, 12 \mathrm{H}), 0.92-0.75(\mathrm{~m}, 1 \mathrm{H}) ;{ }^{13} \mathbf{C}$ NMR (100 MHz, $\left.\mathbf{C D C l}_{3}\right) \delta$ 207.3, 149.1, 137.0, 128.9, 125.1, 51.8, 50.3, 43.8, 43.6, 34.3, 31.3, 26.4, 25.2, 24.2, 21.7; IR (film) 2962, 2933, 2858, 1487, 1444, 1282, 1254, 874, 833, $802 \mathrm{~cm}^{-1}$; HRMS (EI) Calcd for $\mathrm{C}_{19} \mathrm{H}_{29} \mathrm{NS} 303.2021$, Found 303.2023.

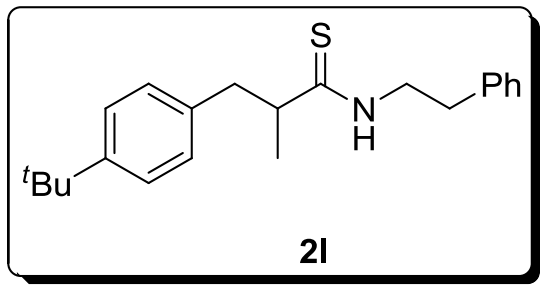

To a Schlenk tube were added lilyaldehyde $(0.3$ mmol, $61.3 \mathrm{mg}), \mathrm{Na}_{2} \mathrm{~S} 9 \mathrm{H}_{2} \mathrm{O}$ (1.05 mmol, $\left.252 \mathrm{mg}\right)$, $\mathrm{K}_{2} \mathrm{~S}_{2} \mathrm{O}_{8}$ (0.54 mmol, $\left.146.0 \mathrm{mg}\right)$, pyridine (1.5 mmol, $118.5 \mathrm{mg}), \quad N$-phenethylformamide (1.5 mmol, $223.5 \mathrm{mg})$ and $\mathrm{H}_{2} \mathrm{O}(0.6 \mathrm{~mL})$, The mixture was stirred at $100{ }^{\circ} \mathrm{C}$ for $24 \mathrm{~h}$. 3-(4-tert-butylphenyl)-2-methyl- $N$-phenethylpropanethioamide $(63.0 \mathrm{mg}, 62 \%)$ was obtained through column chromatography $(\mathrm{PE}: \mathrm{EA}=20: 1)$ as yellow oil, $\mathrm{R}_{\mathrm{f}}=0.50$ (PE: EA = 10: 1). When 2-phenylethanamine (1.5 mmol, $181.5 \mathrm{mg})$ was used instead of $\mathrm{N}$-phenethylformamide in same condition, the product $(7.2 \mathrm{mg}, 7 \%)$ was obtained through column chromatography; ${ }^{1} \mathbf{H}$ NMR (400 $\left.\mathbf{M H z}, \mathbf{C D C l}_{3}\right) \delta$ 7.33-7.27 (m, 3H), 7.26-7.19 (m, 2H), $7.11(\mathrm{~d}, J=8.2 \mathrm{~Hz}, 2 \mathrm{H}), 7.01(\mathrm{~d}, J=6.9 \mathrm{~Hz}, 2 \mathrm{H}), 6.82(\mathrm{br}, 1 \mathrm{H})$, 4.01-3.87 (m, 1H), 3.70-3.62 (m, 1H), $3.00(\mathrm{dd}, J=13.2,8.4 \mathrm{~Hz}, 1 \mathrm{H}), 2.82-2.75(\mathrm{~m}$, 
2H), 2.74-2.64 (m, 1H), 2.62-2.52 (m, 1H), $1.30(\mathrm{~s}, 9 \mathrm{H}), 1.27(\mathrm{~d}, J=6.6 \mathrm{~Hz}, 3 \mathrm{H}) ;{ }^{13} \mathrm{C}$ NMR (100 MHz, $\left.\mathbf{C D C l}_{3}\right) \delta$ 209.2, 149.3, 138.2, 136. 6, 128.7, 128.7, 128.6, 126.7, 125.2, 52.8, 46.2, 42.9, 34.4, 33.7, 31.4, 20.7; IR (film) 3283, 2963, 2868, 1519, 1454, 1268, 1108, 981, $700 \mathrm{~cm}^{-1}$; HRMS (EI) Calcd for $\mathrm{C}_{22} \mathrm{H}_{29} \mathrm{NS}$ 339.2021, Found 339.2024 .

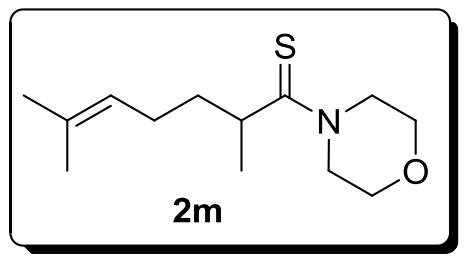

To a Schlenk tube were added 2,6-dimethyl-5-heptenal (0.3 mmol, $42.1 \mathrm{mg}), \mathrm{Na}_{2} \mathrm{~S} 9 \mathrm{H}_{2} \mathrm{O}$ (1.05 mmol, $\left.252 \mathrm{mg}\right)$, $\mathrm{K}_{2} \mathrm{~S}_{2} \mathrm{O}_{8}(0.54 \mathrm{mmol}, 146.0 \mathrm{mg})$, pyridine $(1.5 \mathrm{mmol}$, $118.5 \mathrm{mg}), N$-formylmorpholine (1.5 mmol, $172.5 \mathrm{mg}$ ) and $\mathrm{H}_{2} \mathrm{O} \quad(0.6 \mathrm{~mL})$, The mixture was stirred at $60{ }^{\circ} \mathrm{C}$ for $20 \mathrm{~h}$. 2,6-dimethyl-1-morpholinohept-5-ene-1-thione (48.2 mg, 67\%) was obtained through column chromatography $(\mathrm{PE}: \mathrm{EA}=15: 1)$ as colorless oil, $\mathrm{R}_{\mathrm{f}}=0.5(\mathrm{PE}: \mathrm{EA}=5: 1)$; ${ }^{1}$ H NMR (400 MHz, $\left.\mathbf{C D C l}_{3}\right) \delta$ 5.15-4.96 (m, 1H), 4.54-4.29 (m, 2H), 3.84-3.78 (m, 2H), 3.77-3.75 (m, 2H), 3.73-3.67 (m, 2H), $2.98(\mathrm{dd}, J=13.2,6.6 \mathrm{~Hz}, 1 \mathrm{H}), 2.03-1.86$ $(\mathrm{m}, 3 \mathrm{H}), 1.66(\mathrm{~s}, 3 \mathrm{H}), 1.57(\mathrm{~s}, 3 \mathrm{H}), 1.56-1.49(\mathrm{~m}, 1 \mathrm{H}), 1.21(\mathrm{~d}, J=6.6 \mathrm{~Hz}, 3 \mathrm{H}) ;{ }^{13} \mathrm{C}$ NMR (100 MHz, $\left.\mathbf{C D C l}_{3}\right) \delta 209.9,132.2,123.8,66.6,66.6,50.2,49.7,40.8,37.4$, 25.7, 25.6, 21.2, 17.7; IR (film) 2963, 2926, 2856, 1468, 1432, 1260, 1085, 1017, 795 $\mathrm{cm}^{-1}$; HRMS (EI) Calcd for $\mathrm{C}_{13} \mathrm{H}_{23} \mathrm{NOS} 241.1500$, Found 241.1501.

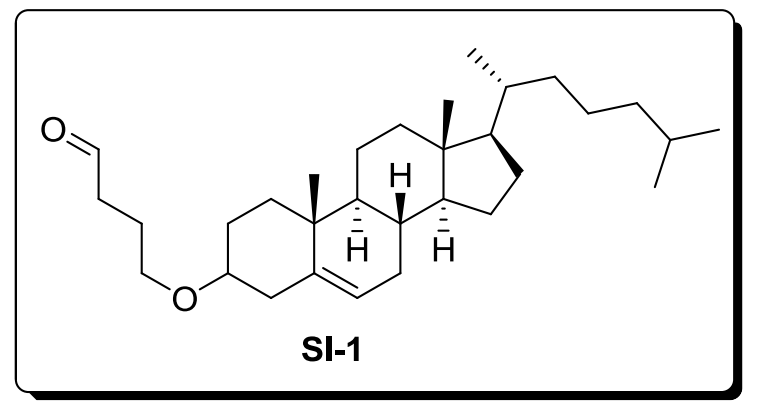

${ }^{1}$ H NMR (400 MHz, $\left.\mathbf{C D C l}_{3}\right) \delta 9.70$ (s, 1H), 5.30-5.22 (m, 1H), 3.50-2.35 $(\mathrm{m}, \quad 2 \mathrm{H}), \quad 3.10-2.98 \quad(\mathrm{~m}, \quad 1 \mathrm{H})$, 2.50-2.38 (m, 2H), 2.33-2.17 (m, $1 \mathrm{H}), 2.15-2.06(\mathrm{~m}, 1 \mathrm{H}), 2.02-1.87$ $(\mathrm{m}, \quad 2 \mathrm{H}), \quad 1.86-1.72(\mathrm{~m}, \quad 5 \mathrm{H})$, 1.57-1.22 (m, 12H), 1.15-0.99 (m, 6H), 0.99-0.87 (m, 6H), 0.85 (d, J = 5.1 Hz, 3H), $0.80(\mathrm{~d}, J=5.6 \mathrm{~Hz}, 6 \mathrm{H}), 0.61(\mathrm{~s}, 3 \mathrm{H}),{ }^{13} \mathbf{C} \mathbf{N M R}\left(\mathbf{1 0 0} \mathbf{M H z}, \mathbf{C D C l}_{3}\right) \delta 201.8,140.6$, 121.4, 79.0, 66.5, 56.6, 56.1, 50.0, 42.2, 40.9, 39.7, 39.4, 38.9, 37.1, 36.7, 36.1, 35.7, $31.8,31.7,28.2,28.1,27.8,24.1,23.8,22.9,22.7,22.4,20.9,19.2,18.6,11.7$; IR 
(KBr) 3460, 2960, 1623, 1418, 1384, 1261, 1107, 804; HRMS (ESI) Calcd for $\mathrm{C}_{31} \mathrm{H}_{52} \mathrm{O}_{2}[\mathrm{M}+\mathrm{H}]^{+}$457.4046, Found 457.4047.

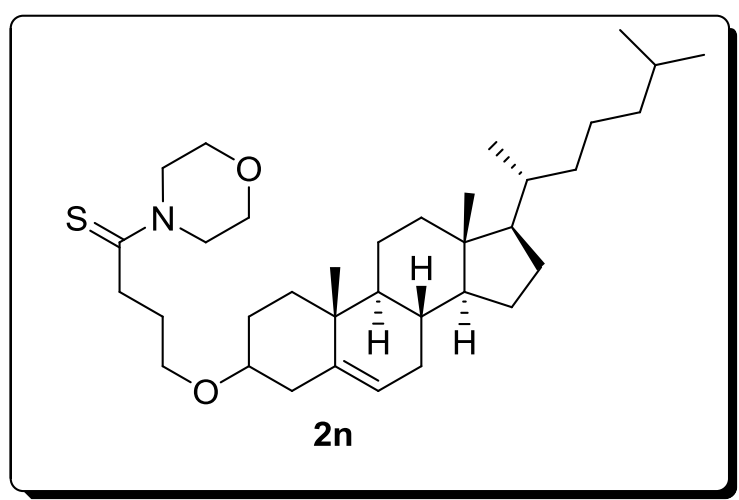

To a Schlenk tube were added SI-1 (0.2 mmol, $91.2 \mathrm{mg}), \mathrm{Na}_{2} \mathrm{~S} 9 \mathrm{H}_{2} \mathrm{O}(0.7$ mmol, $168 \mathrm{mg}), \mathrm{K}_{2} \mathrm{~S}_{2} \mathrm{O}_{8}(0.36 \mathrm{mmol}$, $97.3 \mathrm{mg}$ ), pyridine (1.0 mmol, $79 \mathrm{mg})$, $N$-formylmorpholine $(1.0 \mathrm{mmol}, 115$ $\mathrm{mg})$ and $\mathrm{H}_{2} \mathrm{O} / \mathrm{glycol}(0.3 / 0.3 \mathrm{~mL})$, The mixture was stirred at $100{ }^{\circ} \mathrm{C}$ for $12 \mathrm{~h}$.

2n (60 mg, 54\%) was obtained through column chromatography (PE: EA = 5: 1) as light yellow oil, $\mathrm{R}_{\mathrm{f}}=0.4(\mathrm{PE}: \mathrm{EA}=5: 1) ;{ }^{1} \mathbf{H}$ NMR (400 $\left.\mathbf{M H z}, \mathbf{C D C l}_{3}\right) \delta 5.37-5.29$ (m, 1H), 4.39-4.29 (m, 2H), 3.84-3.69 (m, 6H), $3.52(\mathrm{t}, J=5.9 \mathrm{~Hz}, 2 \mathrm{H}), 3.17-3.08(\mathrm{~m}$, 1H), 2.94 (dd, $J=8.7,6.8 \mathrm{~Hz}, 2 \mathrm{H}), 2.34-2.28$ (m, 1H), 2.20-2.10 (m, 1H), 2.03-1.91 (m, 4H), 1.88-1.79 (m, 3H), 1.60-1.29 (m, 12H), 1.16-1.00 (m, 9H), 0.97 (s, 3H), 0.90 $(\mathrm{d}, J=6.5 \mathrm{~Hz}, 3 \mathrm{H}), 0.86(\mathrm{~d}, J=1.7 \mathrm{~Hz}, 3 \mathrm{H}), 0.84(\mathrm{~d}, J=1.7 \mathrm{~Hz}, 3 \mathrm{H}), 0.66(\mathrm{~s}, 3 \mathrm{H})$; ${ }^{13}$ C NMR (100 MHz, $\left.\mathbf{C D C l}_{3}\right) \delta 203.7,140.7,121.6,79.2,66.6,66.5,66.5,56.7,56.1$, $50.2,50.1,49.9,42.3,40.1,39.8,39.5,39.2,37.2,36.8,36.2,35.7,31.9,31.9,29.8$, 28.5, 28.2, 28.0, 24.3, 23.8, 22.8, 22.5, 21.0, 19.3, 18.7, 11.8; IR (KBr) 3455, 2958, 1633, 1434, 1383, 1261, 1109, 1021, 804; HRMS (ESI) Calcd for $\mathrm{C}_{35} \mathrm{H}_{59} \mathrm{NO}_{2} \mathrm{~S}$ $[\mathrm{M}+\mathrm{H}]^{+}$558.4345, Found 558.4339. 


\section{The Synthetic Procedure and Data for Table 3.}

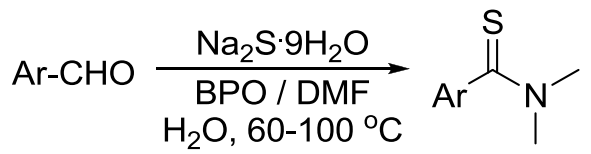

To a Schlenk tube were added aryl aldehyde (0.5 mmol), $\mathrm{Na}_{2} \mathrm{~S} 9 \mathrm{H}_{2} \mathrm{O}(1.75 \mathrm{mmol})$, BPO (1.25 mmol), DMF (2.5 mmol) and $\mathrm{H}_{2} \mathrm{O}(0.5 \mathrm{~mL})$ under air. The mixture was stirred at a certain temperature, and monitored by TLC. When the reaction was finished, the mixture was cooled to rt, quenched by $\mathrm{NH}_{4} \mathrm{Cl}$ (aq.) and extracted with ethyl acetate $(3 * 10 \mathrm{~mL})$. The combined extracts are dried over anhydrous sodium sulfate and concentrated. Purification by column chromatography on silica gel affords product thioamide.

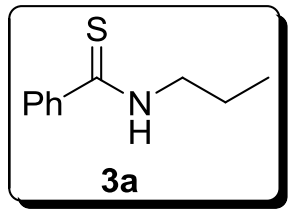

To a Schlenk tube were added benzaldehyde $(0.3 \mathrm{mmol}, 31.8$ $\mathrm{mg}), \mathrm{Na}_{2} \mathrm{~S} \cdot 9 \mathrm{H}_{2} \mathrm{O}(1.05 \mathrm{mmol}, 252 \mathrm{mg}), \mathrm{K}_{2} \mathrm{~S}_{2} \mathrm{O}_{8}(0.54 \mathrm{mmol}, 146.0$ $\mathrm{mg}$ ), pyridine (1.5 mmol, $118.5 \mathrm{mg}), \mathrm{N}$-propylformamide (1.5 mmol, $130.5 \mathrm{mg})$ and $\mathrm{H}_{2} \mathrm{O}(0.6 \mathrm{~mL})$, The mixture was stirred at $100{ }^{\circ} \mathrm{C}$ for $10 \mathrm{~h}$. $N$-propylbenzothioamide ${ }^{[18]}(34.5 \mathrm{mg}, 64 \%)$ was obtained through column chromatography $(\mathrm{PE}: \mathrm{EA}=15: 1)$ as yellow oil. When propan-1-amine $(1.5 \mathrm{mmol}$, $88.5 \mathrm{mg}$ ) was used instead of $\mathrm{N}$-propylformamide in same condition, $N$-propylbenzothioamide $(8.5 \mathrm{mg}, 16 \%)$ was obtained, $\mathrm{R}_{\mathrm{f}}=0.50(\mathrm{PE}: \mathrm{EA}=5: 1) ;{ }^{1} \mathbf{H}$ NMR (400 MHz, CDCl $\left.\mathbf{l}_{3}\right) \delta .72(\mathrm{~d}, J=7.3 \mathrm{~Hz}, 2 \mathrm{H}), 7.60(\mathrm{br}, 1 \mathrm{H}), 7.45$ (t, $J=7.3 \mathrm{~Hz}$ 1H), $7.37(\mathrm{t}, J=7.5 \mathrm{~Hz}, 2 \mathrm{H}), 3.78(\mathrm{dd}, J=13.6,6.4 \mathrm{~Hz}, 2 \mathrm{H}), 1.96-1.68(\mathrm{~m}, 2 \mathrm{H}), 1.04$ $(\mathrm{t}, J=7.4 \mathrm{~Hz}, 3 \mathrm{H}) ;{ }^{13} \mathbf{C}$ NMR (100 MHz, $\left.\mathbf{C D C l}_{3}\right) \delta 199.2,142.0,130.9,128.4,126.5$, 48.4, 21.4, 11.5; IR (film) 3259, 2963, 2931, 2873, 1523, 1486, 1449, 1390, 1347, 1261, 1223, 1064, 1013, 946, 767, $693 \mathrm{~cm}^{-1}$; MS (EI) m/z (\%) 179 (60), 121 (100), 77 $(65)$

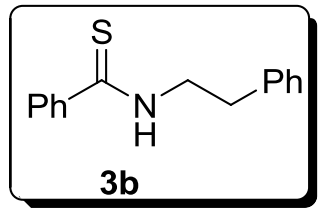

To a Schlenk tube were added benzaldehyde $(0.5 \mathrm{mmol}, 53.0$ $\mathrm{mg}), \mathrm{Na}_{2} \mathrm{~S} \cdot 9 \mathrm{H}_{2} \mathrm{O}$ (3.5 mmol, $\left.840 \mathrm{mg}\right)$, BPO (2.5 mmol, $\left.605 \mathrm{mg}\right)$, $\mathrm{N}$-phenethylformamide (2.5 mmol, $372.5 \mathrm{mg})$ and $\mathrm{H}_{2} \mathrm{O}(1 \mathrm{~mL})$,

The mixture was stirred at $100{ }^{\circ} \mathrm{C}$ for 12 h. $\mathrm{N}$-phenethylbenzothioamide ${ }^{[16]}$ (98.9 mg, 
$82 \%)$ was obtained through column chromatography $(\mathrm{PE}: \mathrm{EA}=15: 1)$ as yellow solid. When 2-phenylethanamine (2.5 mmol, $302.5 \mathrm{mg})$ was used instead of $N$-phenethylformamide in same condition, $N$-phenethylbenzothioamide (9.6 mg, 8\%) was obtained, $\mathrm{R}_{\mathrm{f}}=0.40(\mathrm{PE}: \mathrm{EA}=5: 1) ;{ }^{1} \mathbf{H}$ NMR (400 $\left.\mathbf{M H z}, \mathbf{C D C l}_{3}\right) \delta 7.56-7.54(\mathrm{~m}$, 2H), 7.47 (br, 1H), 7.37-7.33 (m, 1H), 7.28-7.25 (m, 4H), 7.20-7.18 (m, 3H), 4.02 (q, $J=6.3 \mathrm{~Hz}, 2 \mathrm{H}), 3.01(\mathrm{t}, J=6.9 \mathrm{~Hz}, 2 \mathrm{H}) ;{ }^{13} \mathbf{C}$ NMR (100 MHz, $\left.\mathbf{C D C l}_{3}\right) \delta 199.2$, 141.9, 138.2, 131.0, 128.9, 128.7, 128.5, 126.9, 126.5, 47.4, 33.8; IR (film) 3032, 2963, 1531, 1488, 1452, 1261, 1095, 1027, 802, $695 \mathrm{~cm}^{-1}$; MS (EI) $\mathrm{m} / \mathrm{z}(\%) 241(25)$, 121 (40), 104 (100).

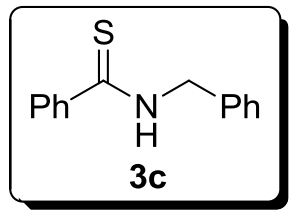

To a Schlenk tube were added benzaldehyde $(0.3 \mathrm{mmol}, 31.8$ mg), $\mathrm{Na}_{2} \mathrm{~S} \cdot 9 \mathrm{H}_{2} \mathrm{O}$ (1.05 mmol, $\left.252 \mathrm{mg}\right), \mathrm{K}_{2} \mathrm{~S}_{2} \mathrm{O}_{8}$ (0.54 mmol, 146.0 $\mathrm{mg})$, pyridine (1.5 mmol, $118.5 \mathrm{mg}), \mathrm{N}$-benzylformamide $(1.5$ mmol, $202.5 \mathrm{mg})$ and $\mathrm{H}_{2} \mathrm{O}(0.6 \mathrm{~mL})$, The mixture was stirred at $100{ }^{\circ} \mathrm{C}$ for $21 \mathrm{~h}$. $N$-benzylbenzothioamide ${ }^{[17]}(38.9 \mathrm{mg}, 57 \%)$ was obtained through column chromatography $(\mathrm{PE}: \mathrm{EA}=10: 1)$ as yellow solid, $\mathrm{R}_{\mathrm{f}}=0.30(\mathrm{PE}: \mathrm{EA}=5: 1)$. When phenylmethanamine (1.5 mmol, $160.5 \mathrm{mg}$ ) was used instead of $N$-benzylformamide in same condition, $N$-benzylbenzothioamide was not obtained through column chromatography; ${ }^{1} \mathbf{H}$ NMR (400 MHz, $\left.\mathbf{C D C l}_{3}\right) \delta$ 7.80-7.67 (m, 3H), 7.51-7.43 (m, 1H), 7.43-7.34 (m, 7H), $5.00(\mathrm{~d}, J=5.1 \mathrm{~Hz}, 2 \mathrm{H}) ;{ }^{13} \mathbf{C}$ NMR (100 MHz, $\left.\mathbf{C D C l}_{3}\right) \delta$ 199.1, 141.6, 136.2, 131.1, 129.0, 128.5, 128.4, 128.2, 126.7, 51.1; IR (film) 2963, 2925, 1521, 1451, 1380, 1262, 1097, 1026, 802, $696 \mathrm{~cm}^{-1}$; MS (EI) $\mathrm{m} / \mathrm{z}(\%) 227$ (60), $121(50), 91(90)$.

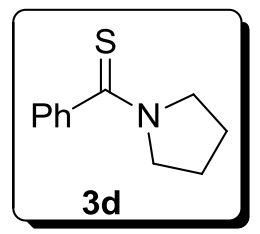

To a Schlenk tube were added benzaldehyde $(0.3 \mathrm{mmol}, 31.8 \mathrm{mg})$, $\mathrm{Na}_{2} \mathrm{~S} 9 \mathrm{H}_{2} \mathrm{O}$ (1.05 mmol, $\left.252 \mathrm{mg}\right), \mathrm{K}_{2} \mathrm{~S}_{2} \mathrm{O}_{8}(0.54 \mathrm{mmol}, 146.0 \mathrm{mg})$, pyridine (1.5 mmol, $118.5 \mathrm{mg})$, pyrrolidine-1-carbaldehyde (1.5 mmol, $148.5 \mathrm{mg})$ and $\mathrm{H}_{2} \mathrm{O}(0.6 \mathrm{~mL})$, The mixture was stirred at 100 ${ }^{\circ} \mathrm{C}$ for 8 h. phenyl(pyrrolidin-1-yl)methanethione ${ }^{[21]}(49.3 \mathrm{mg}, 86 \%)$ was obtained through column chromatography $(\mathrm{PE}: \mathrm{EA}=15: 1)$ as orange oil. When pyrrolidine 
(1.5 mmol, $106.5 \mathrm{mg}$ ) was used instead of pyrrolidine-1-carbaldehyde in same condition, phenyl(pyrrolidin-1-yl)methanethione $(16.5 \mathrm{mg}, 29 \%)$ was obtained, $\mathrm{R}_{\mathrm{f}}=$ 0.50 (PE: EA = 5: 1); ${ }^{1} \mathbf{H}$ NMR (400 MHz, $\left.\mathbf{C D C l}_{3}\right) \delta$ 7.47-7.28 (m, 5H), $3.96(\mathrm{t}, J=$ $7.1 \mathrm{~Hz}, 2 \mathrm{H}), 3.45(\mathrm{t}, J=6.8 \mathrm{~Hz}, 2 \mathrm{H}), 2.14-2.00(\mathrm{~m}, 2 \mathrm{H}), 1.99-1.92(\mathrm{~m}, 2 \mathrm{H}) ;{ }^{13} \mathrm{C}$ NMR (100 MHz, $\left.\mathbf{C D C l}_{3}\right) \delta 197.2,143.9,128.6,128.2,125.5,53.7,53.3,26.4,24.6$; IR (film) 2963, 2875, 1495, 1449, 1327, 1263, 1078, 1034, 800, 762, $698 \mathrm{~cm}^{-1}$; MS (EI) $m / z(\%) 191(80), 121(80), 77(65)$.

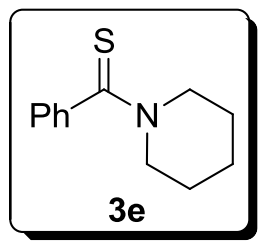

To a Schlenk tube were added benzaldehyde $(0.3 \mathrm{mmol}, 31.8 \mathrm{mg})$, $\mathrm{Na}_{2} \mathrm{~S} 9 \mathrm{H}_{2} \mathrm{O}$ (1.05 mmol, $\left.252 \mathrm{mg}\right), \mathrm{K}_{2} \mathrm{~S}_{2} \mathrm{O}_{8}(0.54 \mathrm{mmol}, 146.0 \mathrm{mg})$, pyridine (1.5 mmol, $118.5 \mathrm{mg})$, piperidine-1-carbaldehyde (1.5 mmol, $169.5 \mathrm{mg})$ and $\mathrm{H}_{2} \mathrm{O}(0.6 \mathrm{~mL})$, The mixture was stirred at 100 ${ }^{\circ} \mathrm{C}$ for 4 h. phenyl(piperidin-1-yl)methanethione ${ }^{[3]}(40.1 \mathrm{mg}, 65 \%)$ was obtained through column chromatography $(\mathrm{PE}: \mathrm{EA}=15: 1)$ as yellow oil. When piperidine (1.5 mmol, $127.5 \mathrm{mg}$ ) was used instead of piperidine-1-carbaldehyde in same condition, phenyl(piperidin-1-yl)methanethione $(10.4 \mathrm{mg}, 17 \%)$ was obtained, $\mathrm{R}_{\mathrm{f}}=0.60(\mathrm{PE}$ : $\mathrm{EA}=5: 1) ;{ }^{1} \mathbf{H}$ NMR (400 MHz, $\left.\mathbf{C D C l}_{3}\right) \delta$ 7.33-7.19 (m, 5H), $4.30(\mathrm{t}, J=5.2 \mathrm{~Hz}$, 2H), $3.46(\mathrm{t}, J=5.6 \mathrm{~Hz} 2 \mathrm{H}), 1.77(\mathrm{dt}, J=11.1,5.5 \mathrm{~Hz}, 2 \mathrm{H}), 1.70(\mathrm{dt}, J=11.3,5.4 \mathrm{~Hz}$, 2H), $1.52(\mathrm{dt}, J=11.5,5.7 \mathrm{~Hz}, 2 \mathrm{H}) ;{ }^{13} \mathbf{C}$ NMR (100 MHz, CDCl 3 ) $\delta$ 199.5, 143.3, 128.3, 128.3, 125.3, 53.1, 50.5, 26.8, 25.4, 24.1; IR (film) 2939, 2857, 1494, 1478, 1444, 1294, 1261, 1244, 1136, 1013, 801, 759, $698 \mathrm{~cm}^{-1}$; MS (EI) $\mathrm{m} / \mathrm{z}$ (\%) 205 (80), $204(100), 121(95)$.

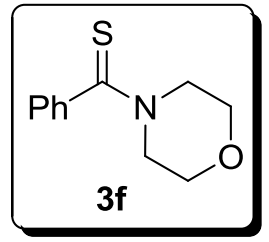

To a Schlenk tube were added benzaldehyde $(0.5 \mathrm{mmol}, 53.0 \mathrm{mg})$, $\mathrm{Na}_{2} \mathrm{~S} 9 \mathrm{H}_{2} \mathrm{O}$ (3.5 mmol, $\left.840 \mathrm{mg}\right)$, BPO (2.5 mmol, $\left.605 \mathrm{mg}\right)$, $N$-formylmorpholine (2.5 mmol, $287.5 \mathrm{mg})$ and $\mathrm{H}_{2} \mathrm{O}(1 \mathrm{~mL})$, The mixture was stirred at $100 \quad{ }^{\circ} \mathrm{C}$ for $12 \mathrm{~h}$. morpholino(phenyl)methanethione ${ }^{[16]}(77.4 \mathrm{mg}, 75 \%)$ was obtained through column chromatography $(\mathrm{PE}: \mathrm{EA}=15: 1)$ as yellow liquid. When morpholine $(2.5 \mathrm{mmol}$, $217.5 \mathrm{mg}$ ) was used instead of $N$-formylmorpholine in same condition, 
morpholino(phenyl)methanethione $(14.4 \mathrm{mg}, 14 \%)$ was obtained, $\mathrm{R}_{\mathrm{f}}=0.50(\mathrm{PE}: \mathrm{EA}=$ 5: 1); ${ }^{1}$ H NMR (400 MHz, $\mathbf{C D C l}_{3}$ ) $\delta$ 7.29-7.20 (m, 5H), 4.53-4.24 (m, 2H), 3.95-3.72 (m, 2H), 3.56-3.54 (m, 4H); ${ }^{13} \mathbf{C}$ NMR (100 MHz, $\left.\mathbf{C D C l}_{3}\right) \delta$ 201.0, 142.5, 128.8, 128.5, 125.8, 66.7, 66.5, 52.5, 49.5; IR (film) 2962, 2923, 2853, 1495, 1479, 1434 , 1291, 1260, 1228, 1112, 1026, 873, 803, 760, $696 \mathrm{~cm}^{-1}$; MS (EI) $\mathrm{m} / \mathrm{z}(\%) 207$ (65), $121(100)$.

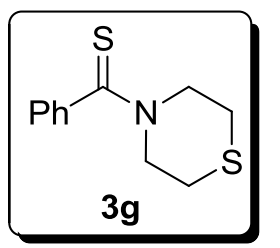

To a Schlenk tube were added benzaldehyde $(0.3 \mathrm{mmol}, 31.8 \mathrm{mg})$, $\mathrm{Na}_{2} \mathrm{~S} 9 \mathrm{H}_{2} \mathrm{O}$ (1.05 mmol, $\left.252 \mathrm{mg}\right), \mathrm{K}_{2} \mathrm{~S}_{2} \mathrm{O}_{8}(0.54 \mathrm{mmol}, 146.0 \mathrm{mg})$, pyridine (1.5 mmol, $118.5 \mathrm{mg}$ ), thiomorpholine-4-carbaldehyde (1.5 mmol, $196.5 \mathrm{mg})$ and $\mathrm{H}_{2} \mathrm{O}(0.6 \mathrm{~mL})$, The mixture was stirred at 100 ${ }^{\circ} \mathrm{C}$ for 8 h. phenyl(thiomorpholino)methanethione $(44.8 \mathrm{mg}, 67 \%)$ was obtained through column chromatography $(\mathrm{PE}: \mathrm{EA}=15: 1)$ as yellow oil. When thiomorpholine $(1.5 \mathrm{mmol}, 154.5 \mathrm{mg})$ was used instead of thiomorpholine-4-carbaldehyde in same condition, phenyl(thiomorpholino)methanethione $(20.0 \mathrm{mg}, 30 \%)$ was obtained, $\mathrm{R}_{\mathrm{f}}=0.50$ (PE: $\mathrm{EA}=5:$ 1); ${ }^{1} \mathbf{H}$ NMR (400 MHz, $\left.\mathbf{C D C l}_{3}\right) \delta$ 7.34-7.22 (m, 3H), 7.20-7.12 (m, 2H), 4.78-4.48 (m, 2H), 3.78-3.75(m, 2H), 2.93-2.72 (m, 2H), 2.65-2.37 (m, 2H); ${ }^{13} \mathrm{C}$ NMR (100 MHz, $\left.\mathbf{C D C l}_{3}\right) \delta$ 201.3, 142.8, 128.6, 128.5, 125.3, 54.4, 51.8, 28.4, 27.2; IR (film) 3053, 2961, 2910, 1470, 1433, 1361, 1296, 1236, 1189, 1037, 950, 800, 758, $698 \mathrm{~cm}^{-1}$; HRMS (EI) Calcd for $\mathrm{C}_{11} \mathrm{H}_{13} \mathrm{NS}_{2}$ 223.0489, Found 223.0487.

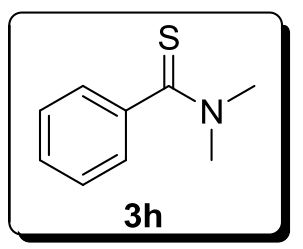

To a Schlenk tube were added benzaldehyde $(0.5 \mathrm{mmol}, 53.1 \mathrm{mg})$, $\mathrm{Na}_{2} \mathrm{~S} 9 \mathrm{H}_{2} \mathrm{O}$ (1.75 mmol, $420 \mathrm{mg}$ ), BPO (1.25 mmol, $\left.302.5 \mathrm{mg}\right)$, DMF (2.5 mmol, $182.5 \mathrm{mg})$ and $\mathrm{H}_{2} \mathrm{O}(0.5 \mathrm{~mL})$, The mixture was stirred at $100{ }^{\circ} \mathrm{C}$ for 12 h. $N, N$-dimethylbenzothioamide ${ }^{[6]}(71.3$ $\mathrm{mg}, 86 \%)$ was obtained through column chromatography $(\mathrm{PE}: \mathrm{EA}=15: 1)$ as yellow oil, $\mathrm{R}_{\mathrm{f}}=0.50$ (PE: EA = 5: 1); ${ }^{1} \mathbf{H}$ NMR (400 MHz, $\left.\mathbf{C D C l}_{3}\right) \delta$ 7.37-7.28 (m, 5H), 3.60 (s, 3H), 3.16 (s, 3H); ${ }^{\mathbf{1 3}} \mathbf{C}$ NMR (100 MHz, $\left.\mathbf{C D C l}_{3}\right) \delta$ 200.0, 142.6, 127.8, 127.5, 
125.0, 43.5, 42.5; IR (film) 3055, 2932, 1514, 1485, 1442, 1390, 1292, 1139, 760, $698 \mathrm{~cm}^{-1}$; MS (EI) $\mathrm{m} / \mathrm{z}(\%) 165$ (65), 164 (85), 121 (100), 77 (70).

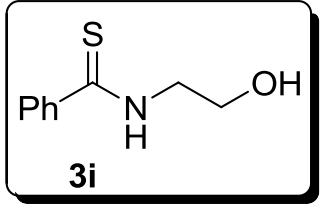

To a Schlenk tube were added benzaldehyde $(0.3 \mathrm{mmol}, 31.8$ $\mathrm{mg}), \mathrm{Na}_{2} \mathrm{~S} 9 \mathrm{H}_{2} \mathrm{O}(1.05 \mathrm{mmol}, 252 \mathrm{mg}), \mathrm{K}_{2} \mathrm{~S}_{2} \mathrm{O}_{8}(0.54 \mathrm{mmol}$, $146.0 \mathrm{mg}), \quad$ pyridine $(1.5 \mathrm{mmol}, \quad 118.5 \mathrm{mg})$, $N$-(2-hydroxyethyl)formamide (1.5 mmol, $133.5 \mathrm{mg}$ ) and $\mathrm{H}_{2} \mathrm{O}$ $\left.\begin{array}{lllllllll} & 0.6 \mathrm{~mL}\end{array}\right)$ The mixture was stirred at $100 \quad{ }^{\circ} \mathrm{C}$ for $5 \mathrm{~h}$. $N$-(2-hydroxyethyl)benzothioamide ${ }^{[19]}$ (40.2 mg, 74\%) was obtained through column chromatography $(\mathrm{PE}: \mathrm{EA}=5: 1)$ as light yellow solid, $\mathrm{R}_{\mathrm{f}}=0.30(\mathrm{PE}: \mathrm{EA}=2: 1)$. When 2-aminoethanol (1.5 mmol, $91.5 \mathrm{mg})$ was used instead of $N$-(2-hydroxyethyl)formamide in same condition, $N$-(2-hydroxyethyl)benzothioamide was not obtained through column chromatography; ${ }^{1} \mathbf{H}$ NMR (400 $\left.\mathbf{M H z}, \mathbf{C D C l}_{3}\right) \delta$ 8.08 (br, 1H), 7.78-7.75 (m, 2H), 7.48-7.45 (m, 1H), 7.40-7.37 (m, 2H), 4.02-4.00 (m, 2H), 3.97-3.96 (m, 2H), 2.12 (br, 1H); ${ }^{13}$ C NMR (100 MHz, CDCl $) \delta$ 199.9, 141.7, 131.2, 128.5, 126.7, 60.6, 48.5; IR (film) 3367, 3263, 2963, 1528, 1450, 1262, 1098, 1026, 802, $696 \mathrm{~cm}^{-1}$; MS (EI) $\mathrm{m} / z(\%) 181$ (70), 121 (100).

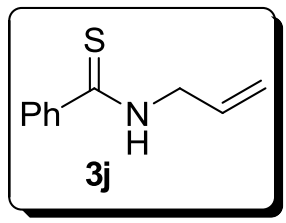

To a Schlenk tube were added benzaldehyde $(0.3 \mathrm{mmol}, 31.8 \mathrm{mg})$, $\mathrm{Na}_{2} \mathrm{~S} 9 \mathrm{H}_{2} \mathrm{O}$ (1.05 mmol, $\left.252 \mathrm{mg}\right), \mathrm{K}_{2} \mathrm{~S}_{2} \mathrm{O}_{8}(0.54 \mathrm{mmol}, 146.0 \mathrm{mg})$, pyridine (1.5 mmol, $118.5 \mathrm{mg}), \mathrm{N}$-allylformamide $(1.5 \mathrm{mmol}$, $127.5 \mathrm{mg})$ and $\mathrm{H}_{2} \mathrm{O}(0.6 \mathrm{~mL})$, The mixture was stirred at $100{ }^{\circ} \mathrm{C}$ for 2 h. $N$-allylbenzothioamide ${ }^{[20]}(39.9 \mathrm{mg}, 75 \%)$ was obtained through column chromatography $(\mathrm{PE}: \mathrm{EA}=15: 1)$ as light yellow oil, $\mathrm{R}_{\mathrm{f}}=0.50(\mathrm{PE}: \mathrm{EA}=5: 1)$. When prop-2-en-1-amine (1.5 mmol, $85.5 \mathrm{mg})$ was used instead of $\mathrm{N}$-allylformamide in same condition, $N$-allylbenzothioamide was not obtained through column chromatography; ${ }^{1} \mathbf{H}$ NMR (400 MHz, $\left.\mathbf{C D C l}_{3}\right) \delta 7.73(\mathrm{~d}, J=7.8 \mathrm{~Hz}, 2 \mathrm{H}), 7.65$ (br, 1H), $7.45(\mathrm{t}, J=7.3 \mathrm{~Hz}, 1 \mathrm{H}), 7.37(\mathrm{t}, J=7.7 \mathrm{~Hz}, 2 \mathrm{H}), 6.09-5.92(\mathrm{~m}, 1 \mathrm{H}), 5.31(\mathrm{dd}, J=$ 22.0, $13.7 \mathrm{~Hz}, 2 \mathrm{H}), 4.45(\mathrm{td}, J=5.9,1.1 \mathrm{~Hz}, 2 \mathrm{H}) ;{ }^{13} \mathbf{C}$ NMR (100 MHz, $\left.\mathbf{C D C l}_{3}\right) \delta$ 199.3, 141.7, 131.8, 131.1, 128.5, 126.6, 118.7, 49.0; IR (film) 3260, 3080, 3028, 
2963, 2911, 1517, 1486, 1448, 1374, 1261, 1218, 1066, 1029, 933, 800, 769, $692 \mathrm{~cm}^{-1}$; MS (EI) $m / z(\%) 177$ (20), 162 (100), 121 (60), 77 (60);

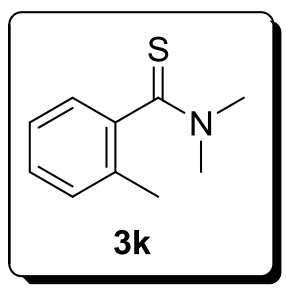

To a Schlenk tube were added 2-methylbenzaldehyde $(0.5 \mathrm{mmol}$, $60.1 \mathrm{mg}), \mathrm{Na}_{2} \mathrm{~S} \cdot 9 \mathrm{H}_{2} \mathrm{O}$ (1.75 mmol, $420 \mathrm{mg}$ ), BPO (1.25 mmol, $302.5 \mathrm{mg})$, DMF (2.5 mmol, $182.5 \mathrm{mg})$ and $\mathrm{H}_{2} \mathrm{O}(0.5 \mathrm{~mL})$, The mixture was stirred at $\quad 60 \quad{ }^{\circ} \mathrm{C} \quad$ for $12 \quad \mathrm{~h}$. $N, N, 2$-trimethylbenzothioamide ${ }^{[6]}(69.0 \mathrm{mg}, 77 \%)$ was obtained through column chromatography $(\mathrm{PE}: \mathrm{EA}=15: 1)$ as yellow oil, $\mathrm{R}_{\mathrm{f}}=0.50(\mathrm{PE}: \mathrm{EA}=5: 1) ;{ }^{1} \mathbf{H} \mathrm{NMR}$ $\left(400 \mathrm{MHz}, \mathbf{C D C l}_{3}\right) \delta 7.33-7.27(\mathrm{~m}, 1 \mathrm{H}), 7.18(\mathrm{~d}, J=5.9 \mathrm{~Hz}, 2 \mathrm{H}), 7.12(\mathrm{~d}, J=7.6 \mathrm{~Hz}$, 1H), 3.64 (s, 3H), 3.21 (s, 3H), 2.40 (s, 3H); ${ }^{13}$ C NMR (100 MHz, CDCl 3 ) $\delta$ 201.4, 143.3 138.0, 129.2, 128.1, 126.2, 122.5, 44.0, 43.0, 21.2; IR (film) 3033, 2929, 1513, 1453, 1390, 1296, 1139, 1024, 787, $702 \mathrm{~cm}^{-1}$; MS (EI) m/z (\%) 179 (70), 178 (85), 135 (100), 91 (65).

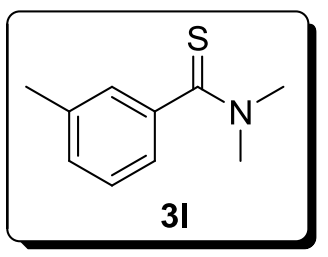

To a Schlenk tube were added 3-methylbenzaldehyde $(0.5$ mmol, $60.0 \mathrm{mg}), \mathrm{Na}_{2} \mathrm{~S} 9 \mathrm{H}_{2} \mathrm{O}(3.5 \mathrm{mmol}, 840 \mathrm{mg})$, BPO (2.5 mmol, $605 \mathrm{mg})$, DMF (5 mmol, $365 \mathrm{mg})$ and $\mathrm{H}_{2} \mathrm{O}(1 \mathrm{~mL})$, The mixture was stirred at $60{ }^{\circ} \mathrm{C}$ for 12 h. $N, N, 3$-trimethylbenzothioamide ${ }^{[6]}(71.6 \mathrm{mg}, 80 \%)$ was obtained through column chromatography (PE: EA $=15: 1)$ as yellow oil, $\mathrm{R}_{\mathrm{f}}=0.50(\mathrm{PE}: \mathrm{EA}=5: 1) ;{ }^{1} \mathbf{H} \mathbf{N M R}$ $\left(400 \mathrm{MHz}, \mathbf{C D C l}_{3}\right) \delta$ 7.35-7.25 (m, 1H), $7.18(\mathrm{~d}, J=6.0 \mathrm{~Hz}, 2 \mathrm{H}), 7.12(\mathrm{~d}, J=7.5 \mathrm{~Hz}$, 1H), 3.64 (s, 3H), 3.21 (s, 3H), 2.40 (s, 3H); ${ }^{13} \mathbf{C}$ NMR (100 MHz, CDCl $) \delta 201.2$, 143.2, 138.0, 129.1, 128.0, 126.1, 122.4, 44.0, 43.0, 21.2; IR (film) 3031, 2961, 2931, 1583, 1453, 1389, 1296, 1260, 1138, 1022, 786, $702 \mathrm{~cm}^{-1}$; MS (EI) $\mathrm{m} / \mathrm{z}(\%) 179$ (80), $178(90), 135$ (100), $91(55)$.

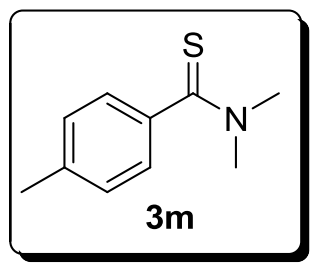

To a Schlenk tube were added 4-methylbenzaldehyde (0.5 mmol, $60.1 \mathrm{mg}), \mathrm{Na}_{2} \mathrm{~S} 9 \mathrm{H}_{2} \mathrm{O}(1.75 \mathrm{mmol}, 420 \mathrm{mg})$, BPO (1.25 
mmol, $302.5 \mathrm{mg})$, DMF (2.5 mmol, $182.5 \mathrm{mg})$ and $\mathrm{H}_{2} \mathrm{O}(0.5 \mathrm{~mL})$, The mixture was stirred at $60{ }^{\circ} \mathrm{C}$ for $12 \mathrm{~h}$. N,N,4-trimethylbenzothioamide ${ }^{[6]}(58.2 \mathrm{mg}, 65 \%)$ was obtained through column chromatography $(\mathrm{PE}: \mathrm{EA}=15: 1)$ as yellow oil, $\mathrm{R}_{\mathrm{f}}=0.50$ $(\mathrm{PE}: \mathrm{EA}=5: 1) ;{ }^{1} \mathbf{H}$ NMR (400 MHz, $\left.\mathbf{C D C l}_{3}\right) \delta 7.19(\mathrm{~d}, J=8.0 \mathrm{~Hz}, 2 \mathrm{H}), 7.13(\mathrm{~d}, J=$ $8.3 \mathrm{~Hz}, 2 \mathrm{H}), 3.57(\mathrm{~d}, J=0.9 \mathrm{~Hz}, 3 \mathrm{H}), 3.16(\mathrm{~d}, J=0.8 \mathrm{~Hz}, 3 \mathrm{H}), 2.33(\mathrm{~s}, 3 \mathrm{H}) ;{ }^{13} \mathrm{C}$ NMR (100 MHz, $\left.\mathbf{C D C l}_{3}\right) \delta 201.4,140.4,138.6,128.8,125.8,44.1,43.2,21.1$; IR (film) 3024, 2924, 2870, 1517, 1502, 1453, 1388, 1291, 1137, 996, 884, $814 \mathrm{~cm}^{-1}$; MS (EI) $m / z(\%) 179$ (75), 178 (100), 135 (98), 91 (65).

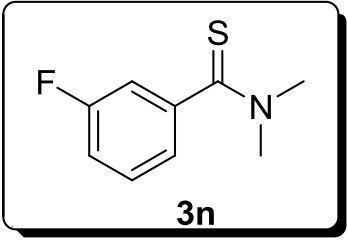

To a Schlenk tube were added 3-fluorobenzaldehyde $(0.5$ mmol, 62.1mg), $\mathrm{Na}_{2} \mathrm{~S} 9 \mathrm{H}_{2} \mathrm{O}$ (1.75 mmol, $\left.420 \mathrm{mg}\right)$, BPO (1.25 mmol, $302.5 \mathrm{mg})$, DMF (2.5 mmol, $182.5 \mathrm{mg})$ and $\mathrm{H}_{2} \mathrm{O}(0.5$ $\mathrm{mL}$ ), The mixture was stirred at $60{ }^{\circ} \mathrm{C}$ for $12 \mathrm{~h}$, 3-fluoro- $N, N$-dimethylbenzothioamide ${ }^{[8]}(59.5 \mathrm{mg}, 65 \%)$ was obtained through column chromatography $(\mathrm{PE}: \mathrm{EA}=15: 1)$ as yellow oil, $\mathrm{R}_{\mathrm{f}}=0.60(\mathrm{PE}: \mathrm{EA}=5: 1) ;{ }^{1} \mathbf{H}$ NMR (400 MHz, CDCl $\left.{ }_{3}\right) \delta$ 7.34-7.28 (m, 1H), 7.08-6.99 (m, 3H), 3.57 (s, 3H), 3.15 $(\mathrm{s}, 3 \mathrm{H}) ;{ }^{\mathbf{1 3}} \mathbf{C}$ NMR (100 MHz, $\left.\mathbf{C D C l}_{\mathbf{3}}\right) \delta 199.2(\mathrm{~s}), 162.2(\mathrm{~d}, J=247.8 \mathrm{~Hz}), 145.0$ (d, $J$ $=7.4 \mathrm{~Hz}), 130.1(\mathrm{~d}, J=8.5 \mathrm{~Hz}), 121.3(\mathrm{~d}, J=3.0 \mathrm{~Hz}), 115.4(\mathrm{~d}, J=21.0 \mathrm{~Hz}), 113.1(\mathrm{~d}$, $J=23.1 \mathrm{~Hz}), 44.0,43.1 ;{ }^{19} \mathbf{F}$ NMR (377 MHz, $\left.\mathbf{C D C l}_{3}\right) \delta-112.10$; IR (film) 3054, 2931, 1583, 1515, 1481, 1428, 1391, 1294, 1137, 826, 786, $695 \mathrm{~cm}^{-1}$; MS (EI) $\mathrm{m} / \mathrm{z}(\%)$ 183 (70), 182 (80), 139 (100), 95 (50).

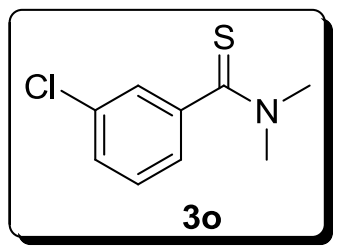

To a Schlenk tube were added 3-chlorobenzaldehyde $(0.5$ mmol, $70.3 \mathrm{mg}), \mathrm{Na}_{2} \mathrm{~S} 9 \mathrm{H}_{2} \mathrm{O}(1.75 \mathrm{mmol}, 420 \mathrm{mg})$, BPO (1.25 mmol, $302.5 \mathrm{mg})$, DMF (2.5 mmol, $182.5 \mathrm{mg})$ and $\mathrm{H}_{2} \mathrm{O}(0.5$ $\mathrm{mL}$ ), The mixture was stirred at $60{ }^{\circ} \mathrm{C}$ for $12 \mathrm{~h}$, 3-chloro- $N, N$-dimethylbenzothioamide (51.9 mg, 52\%) was obtained through column chromatography $(\mathrm{PE}: \mathrm{EA}=15: 1)$ as light yellow oil, $\mathrm{R}_{\mathrm{f}}=0.50(\mathrm{PE}: \mathrm{EA}=5: 1) ;{ }^{1} \mathbf{H}$ NMR (400 MHz, CDCl $\left.)_{3}\right) \delta$ 7.28-7.22 (m, 3H), 7.14-7.12 (m, 1H), 3.54 (s, 3H), 3.13 $(\mathrm{s}, 3 \mathrm{H}) ;{ }^{13} \mathbf{C}$ NMR (100 MHz, $\left.\mathbf{C D C l}_{3}\right) \delta 199.1,144.7,134.2,129.6,128.5,125.8$, 
123.7, 44.0, 43.1; IR (film) 3053, 2961, 2934, 1591, 1564, 1514, 1470, 1389, 1286, 1141, 1078, 1014, 786, 751, $694 \mathrm{~cm}^{-1}$; HRMS (EI) Calcd for $\mathrm{C}_{9} \mathrm{H}_{10} \mathrm{ClNS} 199.0222$, Found 199.0221.

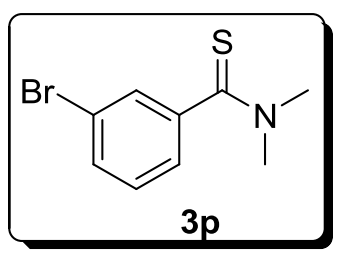

To a Schlenk tube were added 3-bromobenzaldehyde (0.5 mmol, $92.5 \mathrm{mg}), \mathrm{Na}_{2} \mathrm{~S} 9 \mathrm{H}_{2} \mathrm{O}$ (1.75 mmol, $\left.420 \mathrm{mg}\right)$, BPO (1.25 mmol, $302.5 \mathrm{mg})$, DMF (2.5 mmol, $182.5 \mathrm{mg})$ and $\mathrm{H}_{2} \mathrm{O}(0.5$ $\mathrm{mL}$ ), The mixture was stirred at $60{ }^{\circ} \mathrm{C}$ for $12 \mathrm{~h}$. 3-bromo- $N, N$-dimethylbenzothioamide $(69.2 \mathrm{mg}, 57 \%)$ was obtained through column chromatography $(\mathrm{PE}: \mathrm{EA}=15: 1)$ as yellow oil, $\mathrm{R}_{\mathrm{f}}=0.50(\mathrm{PE}: \mathrm{EA}=5: 1)$; ${ }^{\mathbf{1}} \mathbf{H} \mathbf{N M R}$ (400 MHz, $\left.\mathbf{C D C l}_{3}\right) \delta$ 7.46-7.43 (m, 2H), 7.22-7.21 (m, 2H), 3.57 (s, 3H), 3.16 (s, 3H); ${ }^{13}$ C NMR (100 MHz, $\left.\mathbf{C D C l}_{3}\right) \delta$ 199.0, 144.9, 131.5, 129.9, 128.6, 124.2, 122.3, 44.1, 43.1; IR (film) 3051, 2931, 1559, 1514, 1468, 1390, 1285, 1141, 1072, 1010, 894, 785, 728, $693 \mathrm{~cm}^{-1}$; HRMS (EI) Calcd for $\mathrm{C}_{9} \mathrm{H}_{10} \mathrm{BrNS} 242.9717$, Found 242.9715.

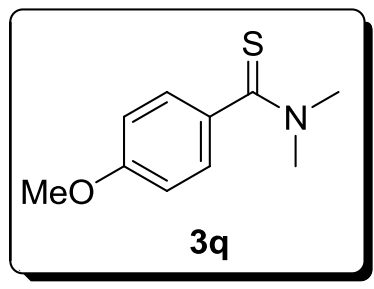

To a Schlenk tube were added 4-methoxybenzaldehyde $(0.5$ mmol, $68.1 \mathrm{mg}), \mathrm{Na}_{2} \mathrm{~S} 9 \mathrm{H}_{2} \mathrm{O}(1.75 \mathrm{mmol}, 420 \mathrm{mg}), \mathrm{BPO}$ (1.25 mmol, $302.5 \mathrm{mg})$, DMF (2.5 mmol, $182.5 \mathrm{mg}$ ) and $\mathrm{H}_{2} \mathrm{O}(0.5 \mathrm{~mL})$, The mixture was stirred at $100{ }^{\circ} \mathrm{C}$ for $12 \mathrm{~h}$. 4-methoxy- $N, N$-dimethylbenzothioamide ${ }^{[6]}(75.0 \mathrm{mg}, 77 \%)$ was obtained through column chromatography $(\mathrm{PE}: \mathrm{EA}=15: 1)$ as yellow oil, $\mathrm{R}_{\mathrm{f}}=0.40(\mathrm{PE}: \mathrm{EA}=5: 1) ;{ }^{1} \mathbf{H}$ NMR (400 MHz, CDCl $) \delta 7.29(\mathrm{~d}, J=8.7 \mathrm{~Hz}, 2 \mathrm{H}), 6.85(\mathrm{~d}, J=8.7 \mathrm{~Hz}, 2 \mathrm{H}), 3.81$ (s, 3H), 3.58 (s, 3H), 3.21 (s, 3H); ${ }^{13}$ C NMR (100 MHz, $\left.\mathbf{C D C l}_{3}\right) \delta 201.3,160.0,135.8$, 127.9, 113.5, 55.4, 44.3, 43.5; IR (film) 2962, 2928, 2857, 1505, 1457, 1391, 1261, 1097, 1031, $801 \mathrm{~cm}^{-1}$; MS (EI) $\mathrm{m} / \mathrm{z}$ (\%) 195 (85), 151 (100), 107 (65).

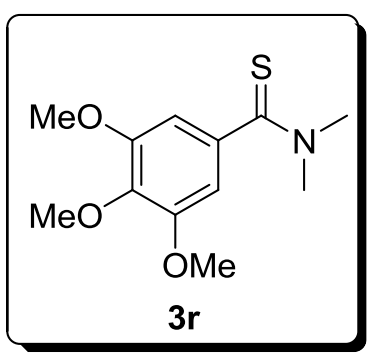

To a Schlenk tube were added 3,4,5-trimethoxybenzaldehyde $(0.5 \mathrm{mmol}, \quad 98.1 \mathrm{mg})$, $\mathrm{Na}_{2} \mathrm{~S} 9 \mathrm{H}_{2} \mathrm{O}$ (1.75 mmol, $420 \mathrm{mg}$ ), BPO (1.25 mmol, 302.5 
$\mathrm{mg})$, DMF (2.5 mmol, $182.5 \mathrm{mg})$ and $\mathrm{H}_{2} \mathrm{O}(0.5 \mathrm{~mL})$, The mixture was stirred at $60{ }^{\circ} \mathrm{C}$ for 12 h. 3,4,5-trimethoxy- $N, N$-dimethylbenzothioamide ${ }^{[7]}(117.0 \mathrm{mg}, 92 \%)$ was obtained through column chromatography (PE: $E A=10: 1)$ as yellow solid, $\mathrm{R}_{\mathrm{f}}=0.40$ $\left(\mathrm{PE}: \mathrm{EA}=3:\right.$ ) ${ }^{1}{ }^{1} \mathbf{H}$ NMR (400 MHz, $\left.\mathbf{C D C l}_{3}\right) \delta 6.51(\mathrm{~s}, 2 \mathrm{H}), 3.84(\mathrm{~s}, 6 \mathrm{H}), 3.83$ (s, 3H), 3.58 (s, 3H), 3.19 (s, 3H); ${ }^{13} \mathbf{C}$ NMR (100 MHz, $\left.\mathbf{C D C l}_{3}\right) \delta 201.0,153.1,138.8$, 138.1, 103.1, 60.8, 56.2, 44.2, 43.2; IR (film) 2962, 2937, 2835, 1581, 1502, 1453, 1409, 1334, 1260, 1235, 1123, 1006, 915, 799, $730 \mathrm{~cm}^{-1}$; MS (EI) $\mathrm{m} / \mathrm{z}(\%) 255(100)$, $211(25)$.

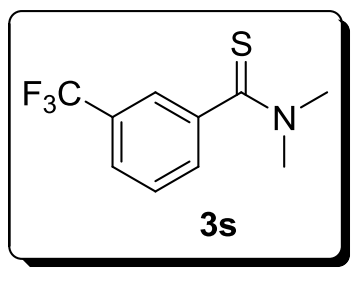

To a Schlenk tube were added 3-(trifluoromethyl)benzaldehyde $(0.5 \mathrm{mmol}, \quad 87.1 \mathrm{mg})$, $\mathrm{Na}_{2} \mathrm{~S} 9 \mathrm{H}_{2} \mathrm{O}(3.5 \mathrm{mmol}, 840 \mathrm{mg})$, BPO (2.5 mmol, $\left.605 \mathrm{mg}\right)$, DMF (5 mmol, $365 \mathrm{mg})$ and $\mathrm{H}_{2} \mathrm{O}(1 \mathrm{~mL})$, The mixture was stirred at $60{ }^{\circ} \mathrm{C}$ for $12 \mathrm{~h}$. $N, N$-dimethyl-3-(trifluoromethyl)benzothioamide ${ }^{[6]}(73.1 \mathrm{mg}$, $63 \%)$ was obtained through column chromatography (PE: EA = 15: 1) as yellow oil, $\mathrm{R}_{\mathrm{f}}=0.40$ (PE: EA = 5: 1); ${ }^{1} \mathbf{H}$ NMR (400 MHz, $\left.\mathbf{C D C l}_{3}\right) \delta$ 7.56-7.47 (m, 4H), 3.59 (s, 3H), 3.15 (s, 3H); ${ }^{13} \mathbf{C}$ NMR (100 MHz, $\left.\mathbf{C D C l}_{3}\right) \delta 199.0,143.8,130.70$ (q, $J=32.6$ $\mathrm{Hz}), 129.0,128.9,125.1$ (q, $J=3.7 \mathrm{~Hz}), 123.5$ (q, $J=271 \mathrm{~Hz}), 122.6$ (q, $J=3.9 \mathrm{~Hz})$, 44.1, 43.1; ${ }^{19} \mathbf{F}$ NMR (376 MHz, $\mathbf{C D C l}_{3}$ ) $\delta$-62.75; IR (film) 2937, 1518, 1393, 1334, 1263, 1125, 1072, 1015, 908, 803, $700 \mathrm{~cm}^{-1}$; MS (EI) m/z (\%) 233 (70), 232 (100), 189 (75), 145 (30).

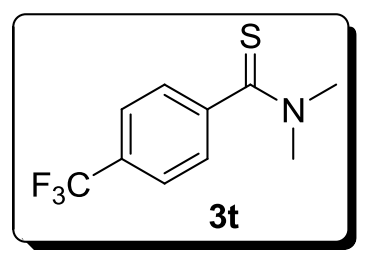

To a Schlenk tube were added 4-(trifluoromethyl)benzaldehyde $(0.5 \mathrm{mmol}, \quad 87.1 \mathrm{mg})$, $\mathrm{Na}_{2} \mathrm{~S} 9 \mathrm{H}_{2} \mathrm{O}$ (3.5 mmol, $\left.840 \mathrm{mg}\right)$, BPO (2.5 mmol, $\left.605 \mathrm{mg}\right)$, DMF (5 mmol, $365 \mathrm{mg}$ ) and $\mathrm{H}_{2} \mathrm{O}(1 \mathrm{~mL})$, The mixture was stirred at $60{ }^{\circ} \mathrm{C}$ for 12 h. $N, N$-dimethyl-4-(trifluoromethyl)benzothioamide ${ }^{[6]}(84.2 \mathrm{mg}$, $72 \%$ ) was obtained through column chromatography (PE: EA = 15: 1) as yellow oil, $\mathrm{R}_{\mathrm{f}}=0.40(\mathrm{PE}: \mathrm{EA}=5: 1) ;{ }^{1} \mathbf{H}$ NMR (400 MHz, $\left.\mathbf{C D C l}_{3}\right) \delta 7.61(\mathrm{~d}, J=8.2 \mathrm{~Hz}, 2 \mathrm{H})$, $7.40(\mathrm{~d}, J=8.1 \mathrm{~Hz}, 2 \mathrm{H}), 3.60(\mathrm{~s}, 3 \mathrm{H}), 3.15(\mathrm{~s}, 3 \mathrm{H}),{ }^{13} \mathbf{C} \mathbf{N M R}\left(\mathbf{1 0 0} \mathbf{M H z}, \mathbf{C D C l}_{3}\right) \delta$ 
199.2, 146.5, 130.4 (q, $J=32.8 \mathrm{~Hz}), 126.0,125.5(\mathrm{q}, J=3.7 \mathrm{~Hz}), 123.7(\mathrm{q}, J=270.5$ $\mathrm{Hz}), 44.0,43.0 ;{ }^{19} \mathbf{F}$ NMR (376 MHz, $\mathbf{C D C l}_{3}$ ) $\delta$-62.83; IR (film) 2962, 2934, 1525, 1504, 1395, 1324, 1294, 1260, 1106, 1066, 1020, 837, 796, $691 \mathrm{~cm}^{-1}$; MS (EI) $\mathrm{m} / \mathrm{z}(\%)$ 233 (70), 232 (100), 189 (70), 145 (40).

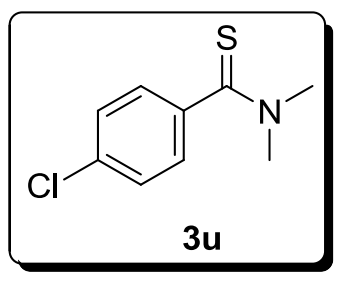

To a Schlenk tube were added 4-chlorobenzaldehyde $(0.5$ mmol, $70.2 \mathrm{mg}$ ), $\mathrm{Na}_{2} \mathrm{~S} 9 \mathrm{H}_{2} \mathrm{O}$ (3.5 mmol, $\left.840 \mathrm{mg}\right)$, BPO (2.5 mmol, $605 \mathrm{mg}$ ), DMF (5 mmol, $365 \mathrm{mg})$ and $\mathrm{H}_{2} \mathrm{O}(1 \mathrm{~mL})$, The mixture was stirred at $100{ }^{\circ} \mathrm{C}$ for $12 \mathrm{~h}$. 4-chloro- $N, N$-dimethylbenzothioamide ${ }^{[9]}(88.5 \mathrm{mg}, 89 \%)$ was obtained through column chromatography $(\mathrm{PE}: \mathrm{EA}=15: 1)$ as yellow oil, $\mathrm{R}_{\mathrm{f}}=0.50(\mathrm{PE}: \mathrm{EA}=5: 1) ;{ }^{1} \mathbf{H}$ NMR (400 MHz, CDCl $)_{3} \delta 7.31(\mathrm{~d}, J=8.6 \mathrm{~Hz}, 2 \mathrm{H}), 7.24(\mathrm{~d}, J=8.6 \mathrm{~Hz}, 2 \mathrm{H}), 3.57(\mathrm{~s}$, 3H), 3.15 (s, 3H); ${ }^{13}$ C NMR (100 MHz, $\mathbf{C D C l}_{3}$ ) $\delta$ 199.3, 141.4, 134.1, 128.2, 127.0, 43.9, 43.0; IR (film) 2962, 2931, 1591, 1513, 1486, 1392, 1288, 1261, 1140, 1090, 996, 823, $729 \mathrm{~cm}^{-1}$; MS (EI) $\mathrm{m} / z$ (\%) 199 (70), 198 (100), 155 (85).

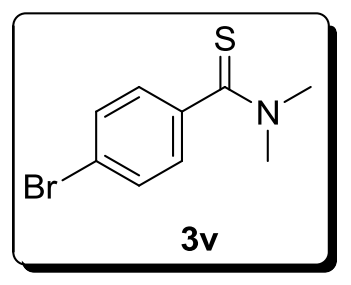

To a Schlenk tube were added 4-bromobenzaldehyde (0.5 mmol, $92.6 \mathrm{mg}), \mathrm{Na}_{2} \mathrm{~S} 9 \mathrm{H}_{2} \mathrm{O}(3.5 \mathrm{mmol}, 840 \mathrm{mg})$, BPO (2.5 mmol, $605 \mathrm{mg})$, DMF (5 mmol, $365 \mathrm{mg})$ and $\mathrm{H}_{2} \mathrm{O}(1 \mathrm{~mL})$, The mixture was stirred at $100 \quad{ }^{\circ} \mathrm{C}$ for $12 \mathrm{~h}$. 4-bromo- $N, N$-dimethylbenzothioamide ${ }^{[10]}(116.0 \mathrm{mg}$, 95\%) was obtained through column chromatography $(\mathrm{PE}: \mathrm{EA}=15: 1)$ as yellow solid, $\mathrm{R}_{\mathrm{f}}=0.50(\mathrm{PE}: \mathrm{EA}=5: 1)$; ${ }^{1} \mathbf{H}$ NMR (400 MHz, $\left.\mathbf{C D C l}_{3}\right) \delta 7.45(\mathrm{~d}, J=8.3 \mathrm{~Hz}, 2 \mathrm{H}), 7.15(\mathrm{~d}, J=8.3 \mathrm{~Hz}, 2 \mathrm{H})$, 3.54 (s, 3H), 3.13 (s, 3H); ${ }^{13} \mathbf{C}$ NMR (100 MHz, $\mathbf{C D C l}_{3}$ ) $\delta$ 199.5, 141.9, 131.3, 127.3, 122.5, 44.0, 43.1; IR (film) 3024, 2961, 2932, 1584, 1514, 1482, 1391, 1287, 1140, 1070, 993, 820, $711 \mathrm{~cm}^{-1}$; MS (EI) $\mathrm{m} / \mathrm{z}$ (\%) 245 (60), 244 (100), 243 (60), 242 (100), 201 (55), 199 (55), $120(80)$. 


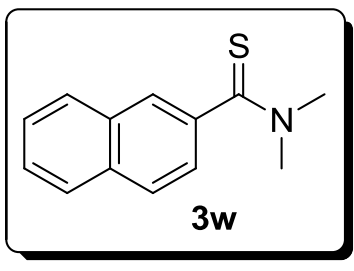

To a Schlenk tube were added 2-naphthaldehyde $(0.5 \mathrm{mmol}$, $78.1 \mathrm{mg}), \mathrm{Na}_{2} \mathrm{~S} \cdot 9 \mathrm{H}_{2} \mathrm{O}(1.75 \mathrm{mmol}, 420 \mathrm{mg})$, BPO (1.25 mmol, $302.5 \mathrm{mg})$, DMF (2.5 mmol, $182.5 \mathrm{mg})$ and $\mathrm{H}_{2} \mathrm{O}(0.5 \mathrm{~mL})$, The mixture was stirred at $60{ }^{\circ} \mathrm{C}$ for $12 \mathrm{~h}$. $N, N$-dimethylnaphthalene-2-carbothioamide ${ }^{[12]}(98.6 \mathrm{mg}, 92 \%)$ was obtained through column chromatography $(\mathrm{PE}: \mathrm{EA}=10: 1)$ as yellow oil, $\mathrm{R}_{\mathrm{f}}=0.40(\mathrm{PE}: \mathrm{EA}=5: 1) ;{ }^{1} \mathbf{H}$ NMR (400 MHz, CDCl $\mathbf{l}_{3} \delta$ 7.84-7.82 (m, 3H), $7.77(\mathrm{~d}, J=1.2 \mathrm{~Hz}, 1 \mathrm{H}), 7.54-7.47(\mathrm{~m}$ 2H), $7.43(\mathrm{dd}, J=8.5,1.7 \mathrm{~Hz}, 1 \mathrm{H}), 3.65(\mathrm{~s}, 3 \mathrm{H}), 3.21(\mathrm{~s}, 3 \mathrm{H}) ;{ }^{13} \mathbf{C}$ NMR (100 MHz, $\left.\mathbf{C D C l}_{3}\right) \delta 201.2,140.5,133.0,132.7,128.3,128.1,127.7,126.7,126.7,124.7,123.9$, 44.2, 43.2; IR (film) 2962, 1515, 1392, 1259, 1087, 1013, 793, $689 \mathrm{~cm}^{-1}$; MS (EI) $\mathrm{m} / \mathrm{z}(\%) 215$ (100), $171(85), 127$ (65).

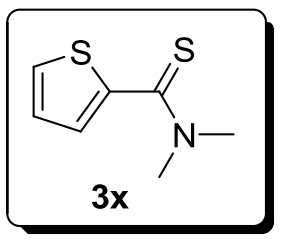

To a Schlenk tube were added thiophene-2-carbaldehyde $(0.5$ mmol, $56.1 \mathrm{mg}), \mathrm{Na}_{2} \mathrm{~S} 9 \mathrm{H}_{2} \mathrm{O}$ (1.75 mmol, $\left.420 \mathrm{mg}\right)$, BPO (1.25 mmol, $302.5 \mathrm{mg})$, DMF (2.5 mmol, $182.5 \mathrm{mg})$ and $\mathrm{H}_{2} \mathrm{O}(0.5 \mathrm{~mL})$, The mixture was stirred at $60{ }^{\circ} \mathrm{C}$ for $12 \mathrm{~h}$. $N, N$-dimethylthiophene-2-carbothioamide ${ }^{[13]}(81.0 \mathrm{mg}, 95 \%)$ was obtained through column chromatography $(\mathrm{PE}: \mathrm{EA}=15: 1)$ as yellow oil, $\mathrm{R}_{\mathrm{f}}=0.60(\mathrm{PE}: \mathrm{EA}=10: 1)$; ${ }^{1}$ H NMR (400 MHz, CDCl $) \delta 7.37(\mathrm{~d}, J=5.1 \mathrm{~Hz}, 1 \mathrm{H}), 7.09(\mathrm{~d}, J=3.6 \mathrm{~Hz}, 1 \mathrm{H})$, $6.95(\mathrm{t}, J=4.4 \mathrm{~Hz}, 1 \mathrm{H}), 3.55(\mathrm{~s}, 3 \mathrm{H}), 3.41(\mathrm{~s}, 3 \mathrm{H}) ;{ }^{13} \mathbf{C}$ NMR $\left(100 \mathbf{M H z}, \mathbf{C D C l}_{3}\right) \delta$ 191.4, 145.1, 129.2, 126.4, 126.3 , 44.5; IR (film) 3071, 2962, 2928, 2869, 1503, 1453, 1386, 1354, 1262, 1124, 1048, 794, $707 \mathrm{~cm}^{-1}$; MS (EI) $\mathrm{m} / \mathrm{z}(\%) 171(65), 127$ $(100)$.

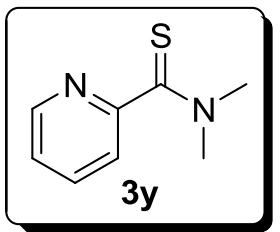

To a Schlenk tube were added picolinaldehyde (0.5 mmol, 53.5 $\mathrm{mg}), \mathrm{Na}_{2} \mathrm{~S} \cdot 9 \mathrm{H}_{2} \mathrm{O}(1.75 \mathrm{mmol}, 420 \mathrm{mg}$ ), BPO (1.25 mmol, 302.5 $\mathrm{mg})$, DMF (2.5 mmol, $182.5 \mathrm{mg})$ and $\mathrm{H}_{2} \mathrm{O}(0.5 \mathrm{~mL})$, The mixture was stirred at $60 \quad{ }^{\circ} \mathrm{C} \quad$ for 12 h. $\quad N, N$ dimethylpyridine-2-carbothioamide ${ }^{[14]}(43.9 \mathrm{mg}, 53 \%)$ was obtained through column chromatography (DCM: $\mathrm{MeOH}=30: 1)$ as light yellow oil, $\mathrm{R}_{\mathrm{f}}=0.40(\mathrm{PE}: \mathrm{EA}=1: 1)$; 
${ }^{1}$ H NMR (400 MHz, $\left.\mathbf{C D C l}_{3}\right) \delta 8.49(\mathrm{~d}, J=4.8 \mathrm{~Hz}, 1 \mathrm{H}), 7.72(\mathrm{t}, J=7.7 \mathrm{~Hz}, 1 \mathrm{H}), 7.56$ $(\mathrm{d}, J=7.9 \mathrm{~Hz}, 1 \mathrm{H}), 7.26-7.19(\mathrm{~m}, 1 \mathrm{H}), 3.59(\mathrm{~s}, 3 \mathrm{H}), 3.17(\mathrm{~s}, 3 \mathrm{H}) ;{ }^{13} \mathbf{C}$ NMR (100 MHz, $\left.\mathbf{C D C l}_{3}\right) \delta 198.0,159.4,148.0,136.9,123.2,123.1,43.7,43.2$; IR (film) 3048, 2961, 2929, 1585, 1524, 1464, 1427, 1393, 1300, 1145, 1014, 785, 745, $702 \mathrm{~cm}^{-1}$; MS (EI) $m / z(\%) 166(45), 123(100), 79(75)$;

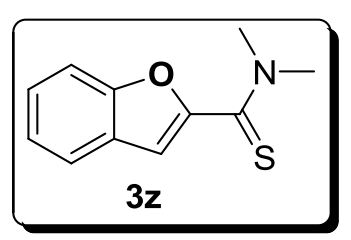

To a Schlenk tube were added benzofuran-2-carbaldehyde ${ }^{[15]}$ (0.5 mmol, $73.1 \mathrm{mg}), \mathrm{Na}_{2} \mathrm{~S} 9 \mathrm{H}_{2} \mathrm{O}$ (1.75 mmol, $\left.420 \mathrm{mg}\right), \mathrm{BPO}$ (1.25 mmol, $302.5 \mathrm{mg}$ ), DMF (2.5 mmol, $182.5 \mathrm{mg}$ ) and $\mathrm{H}_{2} \mathrm{O}$ $(0.5 \mathrm{~mL})$, The mixture was stirred at $60{ }^{\circ} \mathrm{C}$ for $12 \mathrm{~h}$. $\mathrm{N}, \mathrm{N}$-dimethylbenzofuran-2-carbothioamide $(71.7 \mathrm{mg}, 70 \%)$ was obtained through column chromatography $(\mathrm{PE}: \mathrm{EA}=15: 1)$ as yellow oil, $\mathrm{R}_{\mathrm{f}}=0.40(\mathrm{PE}: \mathrm{EA}=10: 1)$; ${ }^{1} \mathbf{H}$ NMR (400 MHz, CDCl 3$) \delta 7.58(\mathrm{~d}, J=7.8 \mathrm{~Hz}, 1 \mathrm{H}), 7.44(\mathrm{~d}, J=8.3 \mathrm{~Hz}, 1 \mathrm{H})$, $7.33(\mathrm{t}, J=7.7 \mathrm{~Hz}, 1 \mathrm{H}), 7.29(\mathrm{~s}, 1 \mathrm{H}), 7.23(\mathrm{t}, J=7.5 \mathrm{~Hz}, 1 \mathrm{H}), 3.55(\mathrm{~s}, 3 \mathrm{H}), 3.42(\mathrm{~s}$, 3H); ${ }^{13} \mathbf{C}$ NMR (100 MHz, $\left.\mathbf{C D C l}_{3}\right) \delta 186.7,154.2,153.3,127.4,126.3,123.5,122.0$, 112.1, 111.6, 44.3, 44.1; IR (film) 2962, 2931, 1567, 1507, 1447, 1390, 1295, 1258, 1127, 1005, 934, 818, $751 \mathrm{~cm}^{-1}$; HRMS (EI) Calcd for $\mathrm{C}_{11} \mathrm{H}_{11} \mathrm{NOS} 205.0561$, Found 205.0560 .

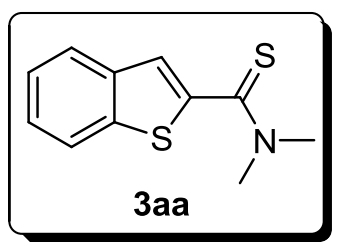

To a Schlenk tube were added benzo[b]thiophene-2-carbaldehyde $(0.3 \mathrm{mmol}, 48.6 \mathrm{mg})$, $\mathrm{Na}_{2} \mathrm{~S} 9 \mathrm{H}_{2} \mathrm{O}$ (1.05 mmol, $\left.252 \mathrm{mg}\right), \mathrm{K}_{2} \mathrm{~S}_{2} \mathrm{O}_{8}(0.54 \mathrm{mmol}, 146 \mathrm{mg})$, DMF (1.5 mmol, $109.5 \mathrm{mg})$, pyridine (1.5 mmol, $118.5 \mathrm{mg}$ ) and $\mathrm{H}_{2} \mathrm{O} \quad(0.6 \mathrm{~mL})$, The mixture was stirred at $100{ }^{\circ} \mathrm{C}$ for $4 \mathrm{~h}$. $N, N$-dimethylbenzo[b]thiophene-2-carbothioamide $(47.8 \mathrm{mg}, 72 \%)$ was obtained through column chromatography $(\mathrm{PE}: \mathrm{EA}=5: 1)$ as yellow solid, $\mathrm{R}_{\mathrm{f}}=0.40(\mathrm{PE}: \mathrm{EA}=$ 5: 1); ${ }^{1} \mathbf{H}$ NMR (400 MHz, $\left.\mathbf{C D C l}_{3}\right) \delta$ 7.82-7.72 (m, 2H), 7.39-7.32 (m, 2H), 7.28 (s, 1H), 3.60 (s, 3H), 3.44 (s, 3H); ${ }^{13}$ C NMR (100 MHz, CDCl 3 ) $\delta$ 191.9, 144.0, 140.4, 138.7, 125.4, 124.8, 124.5, 122.4, 121.9, 44.6, 44.1; IR (KBr) 3436, 2929, 1528, 1509, 1391, 1254, 1130, 843, $761 \mathrm{~cm}^{-1}$; HRMS (EI) Calcd for $\mathrm{C}_{11} \mathrm{H}_{11} \mathrm{NS}_{2} 221.0333$, 
Found 221.0331.

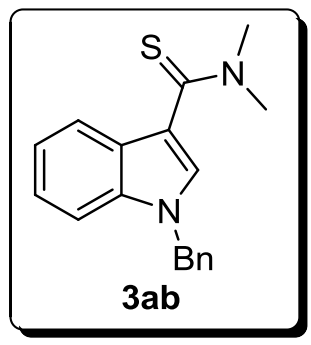

To a Schlenk tube were added $N$-benzylindole-3-carbaldehyde (0.3 mmol, $70.5 \mathrm{mg}), \mathrm{Na}_{2} \mathrm{~S} 9 \mathrm{H}_{2} \mathrm{O}$ (1.05 mmol, $\left.252 \mathrm{mg}\right), \mathrm{K}_{2} \mathrm{~S}_{2} \mathrm{O}_{8}$ (0.54 mmol, $146 \mathrm{mg})$, DMF (1.5 mmol, $109.5 \mathrm{mg})$, pyridine (1.5 mmol, $118.5 \mathrm{mg})$ and $\mathrm{H}_{2} \mathrm{O}(0.6 \mathrm{~mL})$, The mixture was stirred at 100 ${ }^{\circ} \mathrm{C}$ for 12

h. 1-benzyl- $N, N$-dimethyl-1H-indole-3-carbothioamide $(38.0 \mathrm{mg}, 43 \%)$ was obtained through column chromatography $(\mathrm{PE}: \mathrm{EA}=5: 1)$ as light yellow solid, $\mathrm{R}_{\mathrm{f}}=0.60(\mathrm{PE}$ : $\mathrm{EA}=3:$ 1). ${ }^{1} \mathbf{H}$ NMR (400 MHz, $\left.\mathbf{C D C l}_{3}\right) \delta$ 7.64-7.58 (m, 1H), $7.55(\mathrm{~s}, 1 \mathrm{H}), 7.35-7.27$ (m, 4H), 7.23-7.14 (m, 4H), 5.29 (s, 2H), 3.63 (s, 3H), 3.35 (s, 3H); ${ }^{13}$ C NMR (100 MHz, $\left.\mathbf{C D C l}_{3}\right) \delta 194.5,136.2,135.9,131.1,128.8,127.9,127.0,124.9,122.4,121.1$, 120.8, 119.4, 110.3, 50.4, 44.5, 43.6; IR (KBr) 3436, 2926, 1534, 1465, 1388, 1269, 1176, 1125, $747 \mathrm{~cm}^{-1}$; HRMS (EI) Calcd for $\mathrm{C}_{18} \mathrm{H}_{18} \mathrm{~N}_{2} \mathrm{~S} 294.1191$, Found 294.1193.

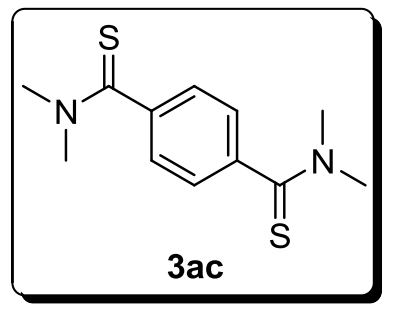

To a Schlenk tube were added 1,4-phthalaldehyde (0.3 mmol, $40.2 \mathrm{mg}$ ), $\mathrm{Na}_{2} \mathrm{~S} 9 \mathrm{H}_{2} \mathrm{O}$ (2.1 mmol, $504 \mathrm{mg}$ ), $\mathrm{K}_{2} \mathrm{~S}_{2} \mathrm{O}_{8}$ (1.08 mmol, $292 \mathrm{mg}$ ), DMF (3 mmol, $219 \mathrm{mg}$ ), pyridine (3 mmol, $237 \mathrm{mg})$ and $\mathrm{H}_{2} \mathrm{O}(1 \mathrm{~mL})$, The mixture was stirred at $100{ }^{\circ} \mathrm{C}$ for 15 h. $N_{l}, N_{1}, N_{4}, N_{4}$-tetramethylbenzene-1,4-bis(carbothioamide) (34.8 mg, 46\%) was obtained through column chromatography $(\mathrm{PE}: \mathrm{EA}=5: 1)$ as yellow oil, $\mathrm{R}_{\mathrm{f}}$ $=0.40\left(\mathrm{PE}: \mathrm{EA}=2:\right.$ 1). ${ }^{1} \mathbf{H}$ NMR (400 MHz, CDCl $) \delta 7.30(\mathrm{~s}, 4 \mathrm{H}), 3.59(\mathrm{~s}, 6 \mathrm{H})$, 3.19 (s, 6H); ${ }^{13} \mathbf{C}$ NMR (100 MHz, $\left.\mathbf{C D C l}_{3}\right) \delta$ 200.1, 143.3, 125.9, 44.3, 43.2; IR (KBr) 3436, 1635, 1385, 1132, $563 \mathrm{~cm}^{-1}$; HRMS (EI) Calcd for $\mathrm{C}_{12} \mathrm{H}_{16} \mathrm{~N}_{2} \mathrm{~S}_{2}$ 252.0755, Found 252.0759.

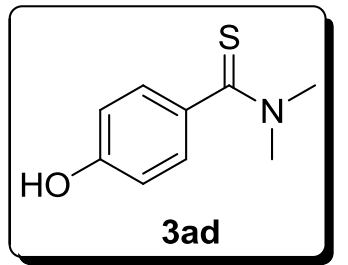

To a Schlenk tube were added 4-hydroxybenzaldehyde $(0.3$ mmol, $36.6 \mathrm{mg}$ ), $\mathrm{Na}_{2} \mathrm{~S} 9 \mathrm{H}_{2} \mathrm{O}$ (1.05 mmol, $\left.252 \mathrm{mg}\right), \mathrm{K}_{2} \mathrm{~S}_{2} \mathrm{O}_{8}$ (0.54 mmol, $146 \mathrm{mg}$ ), DMF (1.5 mmol, $109.5 \mathrm{mg}$ ), pyridine 
(1.5 mmol, $118.5 \mathrm{mg})$ and $\mathrm{H}_{2} \mathrm{O}(0.6 \mathrm{~mL})$, The mixture was stirred at $100{ }^{\circ} \mathrm{C}$ for $7 \mathrm{~h}$. 4-hydroxy- $N, N$-dimethylbenzothioamide ${ }^{[11]}(33.1 \mathrm{mg}, 61 \%)$ was obtained through column chromatography $(\mathrm{PE}: \mathrm{EA}=5: 1)$ as light yellow solid, $\mathrm{R}_{\mathrm{f}}=0.40(\mathrm{PE}: \mathrm{EA}=1$ : 1); ${ }^{1} \mathbf{H}$ NMR (400 MHz, $\left.\mathbf{C D C l}_{3}\right) \delta 7.19(\mathrm{~d}, J=8.5 \mathrm{~Hz}, 2 \mathrm{H}), 6.72(\mathrm{~d}, J=8.5 \mathrm{~Hz}, 2 \mathrm{H})$, 5.62 (br, 1H), 3.59 (s, 3H), 3.20 (s, 3H); ${ }^{\mathbf{1 3}} \mathbf{C}$ NMR (100 MHz, CDCl $) \delta$ 201.4, 156.4, 135.6, 127.9, 115.2, 44.4, 43.6; IR (film) 3123, 2925, 2856, 1607, 1506, 1457, 1390, 1270, 1231, 1138, $883 \mathrm{~cm}^{-1}$; MS (EI) $\mathrm{m} / \mathrm{z}(\%) 181$ (85), 180 (80), 137 (100).

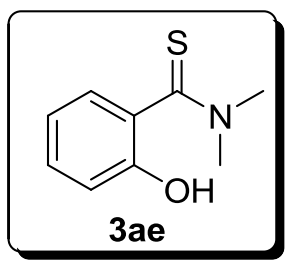

To a Schlenk tube were added salicylaldehyde $(0.3 \mathrm{mmol}, 36.6$ $\mathrm{mg}$ ), $\mathrm{Na}_{2} \mathrm{~S} \cdot 9 \mathrm{H}_{2} \mathrm{O}$ (1.05 mmol, $\left.252 \mathrm{mg}\right), \mathrm{K}_{2} \mathrm{~S}_{2} \mathrm{O}_{8}(0.54 \mathrm{mmol}, 146$ $\mathrm{mg}), \mathrm{DMF}$ (1.5 mmol, $109.5 \mathrm{mg})$, pyridine (1.5 mmol, $118.5 \mathrm{mg})$ and $\mathrm{H}_{2} \mathrm{O}(0.6 \mathrm{~mL})$, The mixture was stirred at $100{ }^{\circ} \mathrm{C}$ for $6 \mathrm{~h}$. 2-hydroxy- $N, N$-dimethylbenzothioamide $(46.2 \mathrm{mg}, 85 \%)$ was obtained through column chromatography $(\mathrm{PE}: \mathrm{EA}=5: 1)$ as light yellow oil, $\mathrm{R}_{\mathrm{f}}=0.35(\mathrm{PE}: \mathrm{EA}=2$ : 1); ${ }^{1}$ H NMR (400 MHz, CDCl 3 ) $\delta 7.95(\mathrm{~s}, 1 \mathrm{H}), 7.12$ (t, $\left.J=7.1 \mathrm{~Hz}, 1 \mathrm{H}\right), 6.99$ (d, $J=$ $7.3 \mathrm{~Hz}, 1 \mathrm{H}), 6.90-6.74$ (m, 2H), 3.48 (s, 3H), 3.13 (s, 4H); ${ }^{13}$ C NMR (100 MHz, $\left.\mathbf{C D C l}_{3}\right) \delta 196.1,152.5,152.5,130.4,127.5,127.5,126.3,119.4,117.4,44.1,42.7 ;$ IR (KBr) 3412, 2963, 1602, 1528, 1448, 1396, 1294, 1142, 1106, 821, $756 \mathrm{~cm}^{-1}$; HRMS (ESI) Calcd for $\mathrm{C}_{9} \mathrm{H}_{11} \mathrm{NOS}[\mathrm{M}+\mathrm{H}]^{+}$182.0640, Found 182.0648.

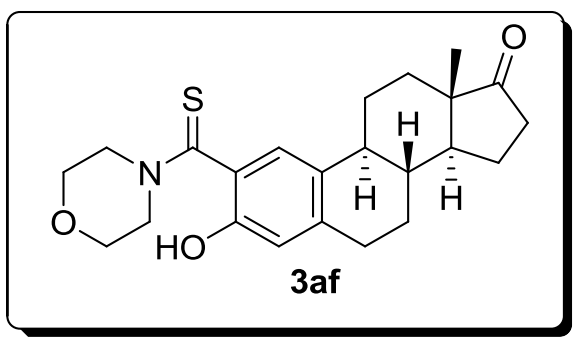

To a Schlenk tube were added 2-formylestrone ${ }^{[22]}(0.3 \mathrm{mmol}, 89.5 \mathrm{mg})$, $\mathrm{Na}_{2} \mathrm{~S} 9 \mathrm{H}_{2} \mathrm{O}(1.05 \mathrm{mmol}, 252 \mathrm{mg}), \mathrm{K}_{2} \mathrm{~S}_{2} \mathrm{O}_{8}(0.54$ mmol, $146 \mathrm{mg}), \mathrm{N}$-formylmorpholine (1.5 mmol, $172.5 \mathrm{mg})$, pyridine (1.5 mmol, $118.5 \mathrm{mg})$ and $\mathrm{H}_{2} \mathrm{O} /$ glycol $(0.3 / 0.3 \mathrm{~mL})$, The mixture was stirred at $100{ }^{\circ} \mathrm{C}$ for $10 \mathrm{~h}$. 3ac $(84.5 \mathrm{mg}$, $71 \%)$ was obtained through column chromatography (PE: EA = 5: 1) as light yellow solid, $\mathrm{R}_{\mathrm{f}}=0.4$ (PE: EA = 3: 1); ${ }^{1} \mathbf{H}$ NMR (400 MHz, $\left.\mathbf{C D C l}_{3}\right) \delta 7.78(\mathrm{br}, 1 \mathrm{H}), 6.88(\mathrm{~s}$, $1 \mathrm{H}), 6.69(\mathrm{~s}, 1 \mathrm{H}), 4.17-3.90(\mathrm{~m}, 2 \mathrm{H}), 3.85-3.65(\mathrm{~m}, 4 \mathrm{H}), 2.83(\mathrm{~d}, J=4.6 \mathrm{~Hz}, 2 \mathrm{H})$, $2.49(\mathrm{dd}, J=18.9,8.6 \mathrm{~Hz}, 1 \mathrm{H}), 2.36-1.85(\mathrm{~m}, 7 \mathrm{H}), 1.67-1.29(\mathrm{~m}, 7 \mathrm{H}), 0.89$ (s, 3H); 
${ }^{13}$ C NMR (100 MHz, $\left.\mathbf{C D C l}_{3}\right) \delta$ 220.8, 197.0, 151.5, 140.2, 131.4, 124.5, 123.1, 117.9, 66. 6, 50.3, 47.8, 43.6, 38.1, 35.7, 31.4, 29.2, 26.2, 25.8, 21.5, 13.8; IR (KBr) 3434, 2962, 2927, 2857, 1735, 1616, 1436, 1261, 1130, 1029, $803 \mathrm{~cm}^{-1}$; HRMS (ESI) Calcd for $\mathrm{C}_{23} \mathrm{H}_{29} \mathrm{NO}_{3} \mathrm{~S}[\mathrm{M}+\mathrm{H}]^{+} 400.1946$, Found 400.1931 .

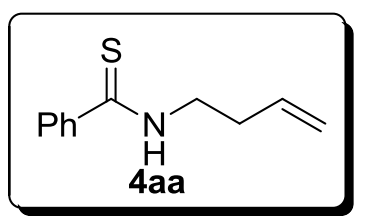

To a Schlenk tube were added benzaldehyde $(0.3 \mathrm{mmol}, 31.8$ $\mathrm{mg}), \mathrm{Na}_{2} \mathrm{~S} 9 \mathrm{H}_{2} \mathrm{O}(1.05 \mathrm{mmol}, 252 \mathrm{mg}), \mathrm{K}_{2} \mathrm{~S}_{2} \mathrm{O}_{8}(0.54 \mathrm{mmol}$, $146.0 \mathrm{mg}), \mathrm{N}$-(but-3-enyl)formamide (1.5 mmol, $148.5 \mathrm{mg}$ ), pyridine $(1.5 \mathrm{mmol}, 118.5 \mathrm{mg})$ and $\mathrm{H}_{2} \mathrm{O}(0.6 \mathrm{~mL})$, The mixture was stirred at $100{ }^{\circ} \mathrm{C}$ for $6 \mathrm{~h}$. $4 \mathbf{a a}(31.4 \mathrm{mg}, 55 \%)$ was obtained through column chromatography (PE: EA = 10: 1) as yellow oil, $\mathrm{R}_{\mathrm{f}}=0.5(\mathrm{PE}: \mathrm{EA}=5: 1) ;{ }^{1} \mathbf{H} \mathbf{N M R}\left(\mathbf{4 0 0} \mathbf{M H z}, \mathbf{C D C l}_{3}\right) \delta 7.71(\mathrm{~d}$, $J=7.7 \mathrm{~Hz}, 2 \mathrm{H}), 7.60(\mathrm{br}, 1 \mathrm{H}), 7.45(\mathrm{t}, J=7.2 \mathrm{~Hz}, 1 \mathrm{H}), 7.38$ (t, $J=7.5 \mathrm{~Hz}, 2 \mathrm{H}), 5.86$ (ddt, $J=17.0,10.1,6.8 \mathrm{~Hz}, 1 \mathrm{H}), 5.20$ (t, $J=13.0 \mathrm{~Hz}, 2 \mathrm{H}), 3.89(\mathrm{dd}, J=12.2,6.1 \mathrm{~Hz}$, 2H), 2.53 (q, $J=6.5 \mathrm{~Hz}, 2 \mathrm{H}) .{ }^{13} \mathbf{C}$ NMR (100 MHz, $\left.\mathbf{C D C l}_{3}\right) \delta 199.1,141.9,134.8$, 131.0, 128.5, 126.5, 118.1, 45.2, 32.2.; IR (KBr) 3443, 2924, 1639, 1525, 1449, 1386 , 1338, 1262, 1090, $695 \mathrm{~cm}^{-1}$ HRMS (EI) Calcd for $\mathrm{C}_{11} \mathrm{H}_{13} \mathrm{NS}$ 191.0769, Found 191.0771.

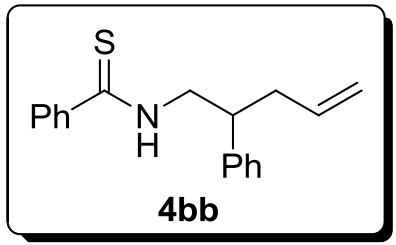

To a Schlenk tube were added benzaldehyde $(0.3 \mathrm{mmol}$, $31.8 \mathrm{mg}), \mathrm{Na}_{2} \mathrm{~S} 9 \mathrm{H}_{2} \mathrm{O}(1.05 \mathrm{mmol}, 252 \mathrm{mg}), \mathrm{K}_{2} \mathrm{~S}_{2} \mathrm{O}_{8}(0.54$ mmol, $146.0 \mathrm{mg}), \mathrm{N}$-(2-phenylpent-4-enyl)formamide $(1.5$ mmol, $283.5 \mathrm{mg}$ ), pyridine ( $1.5 \mathrm{mmol}, 118.5 \mathrm{mg}$ ) and $\mathrm{H}_{2} \mathrm{O}$ (0.6 mL), The mixture was stirred at $100{ }^{\circ} \mathrm{C}$ for $6 \mathrm{~h} .4 \mathbf{a a}(26 \mathrm{mg}, 31 \%)$ was obtained through column chromatography $(\mathrm{PE}: \mathrm{EA}=10: 1)$ as yellow oil, $\mathrm{R}_{\mathrm{f}}=0.5(\mathrm{PE}: \mathrm{EA}=5$ : 1); ${ }^{1} \mathbf{H}$ NMR (400 MHz, $\left.\mathbf{C D C l}_{3}\right) \delta 7.41(\mathrm{~d}, J=7.6 \mathrm{~Hz}, 2 \mathrm{H}), 7.36-7.24(\mathrm{~m}, 4 \mathrm{H}), 7.20$ $(\mathrm{dd}, J=14.6,7.1 \mathrm{~Hz}, 5 \mathrm{H}), 5.74-5.57(\mathrm{~m}, 1 \mathrm{H}), 4.97(\mathrm{dd}, J=24.6,13.6 \mathrm{~Hz}, 2 \mathrm{H})$, 4.35-4.20 (m, 1H), 3.74-3.60 (m, 1H), 3.26-3.10 (m, 1H), $2.43(\mathrm{t}, J=7.0 \mathrm{~Hz}, 2 \mathrm{H}) .{ }^{13} \mathrm{C}$ NMR (100 MHz, $\left.\mathbf{C D C l}_{3}\right) \delta 198.9,141.7,141.4,135.4,130.9,129.0,128.4,127.7$, 127.3, 126.4, 117.1, 51.3, 43.8, 38.6; IR (KBr) 3383, 2924, 1640, 1521, 1489, 1450, 1386, 1335, 1201, 918, 766, $698 \mathrm{~cm}^{-1}$. HRMS (EI) Calcd for $\mathrm{C}_{18} \mathrm{H}_{19} \mathrm{NS} 281.1238$, 
Found 281.1241.

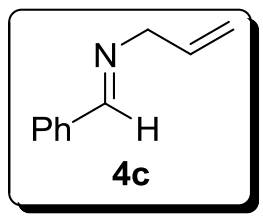

To a three-necked bottle were added freshly distilled benzaldehyde (1.06 g, $10 \mathrm{mmol})$, allyl amine (570 mg, $10 \mathrm{mmol})$ and DCM (20 $\mathrm{mL}$ ). The solution was stirred at room temperature for $20 \mathrm{~h}$. After the reaction, the solvent was removed under vacuum to give orange oil of $N$-benzylideneprop-2-en-1-amine. ${ }^{\mathbf{1}} \mathbf{H}$ NMR (400 $\left.\mathbf{M H z}, \mathbf{C D C l}_{3}\right) \delta 8.29(\mathrm{~s}, 1 \mathrm{H})$, 7.80-7.72 (m, 2H), 7.46-7.38 (m, 3H), 6.08 (ddt, $J=17.2,10.3,5.7 \mathrm{~Hz}, 1 \mathrm{H}), 5.20$ $(\mathrm{ddq}, J=20.2,10.3,1.6 \mathrm{~Hz}, 2 \mathrm{H}), 4.26(\mathrm{ddd}, J=5.6,3.0,1.5 \mathrm{~Hz}, 2 \mathrm{H}) .{ }^{13} \mathbf{C}$ NMR $(\mathbf{1 0 0}$ MHz, $\left.\mathbf{C D C l}_{3}\right) \delta 161.9,136.0,135.7,130.6,128.4,128.0,115.9,63.3$; IR (KBr) 3437, 1636, 1555, 1386, 1130, 708, $565 \mathrm{~cm}^{-1}$; HRMS (EI) Calcd for $\mathrm{C}_{10} \mathrm{H}_{11} \mathrm{~N} 145.0891$, Found 145.0890. 


\section{References}

[1] Nooshabadi, M.; Aghapoor, K.; Darabi, H. R.; Mojtahedi, M. M. Tetrahedron Lett. 1999, 40, 7549; Salim, S. D.; Pathare, S. P.; Akamanchi, K. G. Catal. Commun. 2011, 13,78 .

[2] Yu, H.; Liu, X.; Ding, L.; Yang, Q.; Rong, B.; Gao, A.; Zhao, B.; Yang, H. Tetrahedron Lett. 2013, 54, 3060.

[3] Guntreddi T., Vanjari, R. Singh. K. N. Org. Lett. 2014, 16, 3624.

[4] Reynaud, P. et al Bull. Soc. Chim. Fr. 1965, 3623.

[5] Otten, P. A.; Gen, A. Recl. Trav. Chim. Pays-Bas 1994, 113, 499.

[6] Qu, Y.; Li, Z.; Xiang, H.; Zhou, X. Adv. Synth. Catal. 2013, 355, 3141.

[7] Farina, C.; Pellegata, R.; Pinza, M.; Pifferi, G. Arch. Pharm. 1981, 314, 108.

[8] Creary, X.; Aldridge, T. J. Org. Chem. 1991, 56, 4280.

[9] Kaboudin, B.; Malekzadeh, L. Synlett 2011, 19, 2807.

[10] Amupitan, J. O. Synthesis 1983, 9, 730.

[11] Scheibye, S.; Pedersen B. S.; Lawesson S. O. Bull. Soc. Chim. Belg. 1978, 87, 229.

[12] Berg, U. Acta. Chem. Scand., Ser. B 1976, 30, 695.

[13] Gronowitz, S.; Hornfeldt, A. B.; Temciuc, M. Synthesis 1993, 5, 483.

[14] Wegler, R.; Kühle. E.; Schäfer W. Angew. Chem. 1958, 70, 351.

[15] Gigant, N.; Claveau, E.; Bouyssou, P.; Gillaizeau, I. Org. Lett. 2012, 14, 844.

[16] Nguyen, T. B.; Ermolenko, L.; Al-Mourabit, A. Org. Lett. 2012, 14, 4274.

[17] Shibahara, F.; Sugiura, R.; Murai, T. Org. Lett. 2009, 11, 3064.

[18] Xu, H.; Deng, H.; Li, Z.; Xiang, H.; Zhou, X. Eur. J. Org. Chem. 2013, 31, 7054.

[19] Doszczak, L.; Rachon, J. Chem. Commun. 2000, 21, 2093.

[20] Engman, L. J. Org. Chem. 1991, 56, 3425.

[21] Wang, X.; Ji, M.; Lim, S. Jang, H. Y. J. Org. Chem. 2014, 79, 7256.

[22] Akselsen, O.; Hansen, T. Tetrahedron 2011, 67, 7738. 
VII. NMR Spectra

Qa:

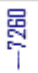

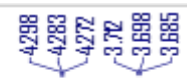
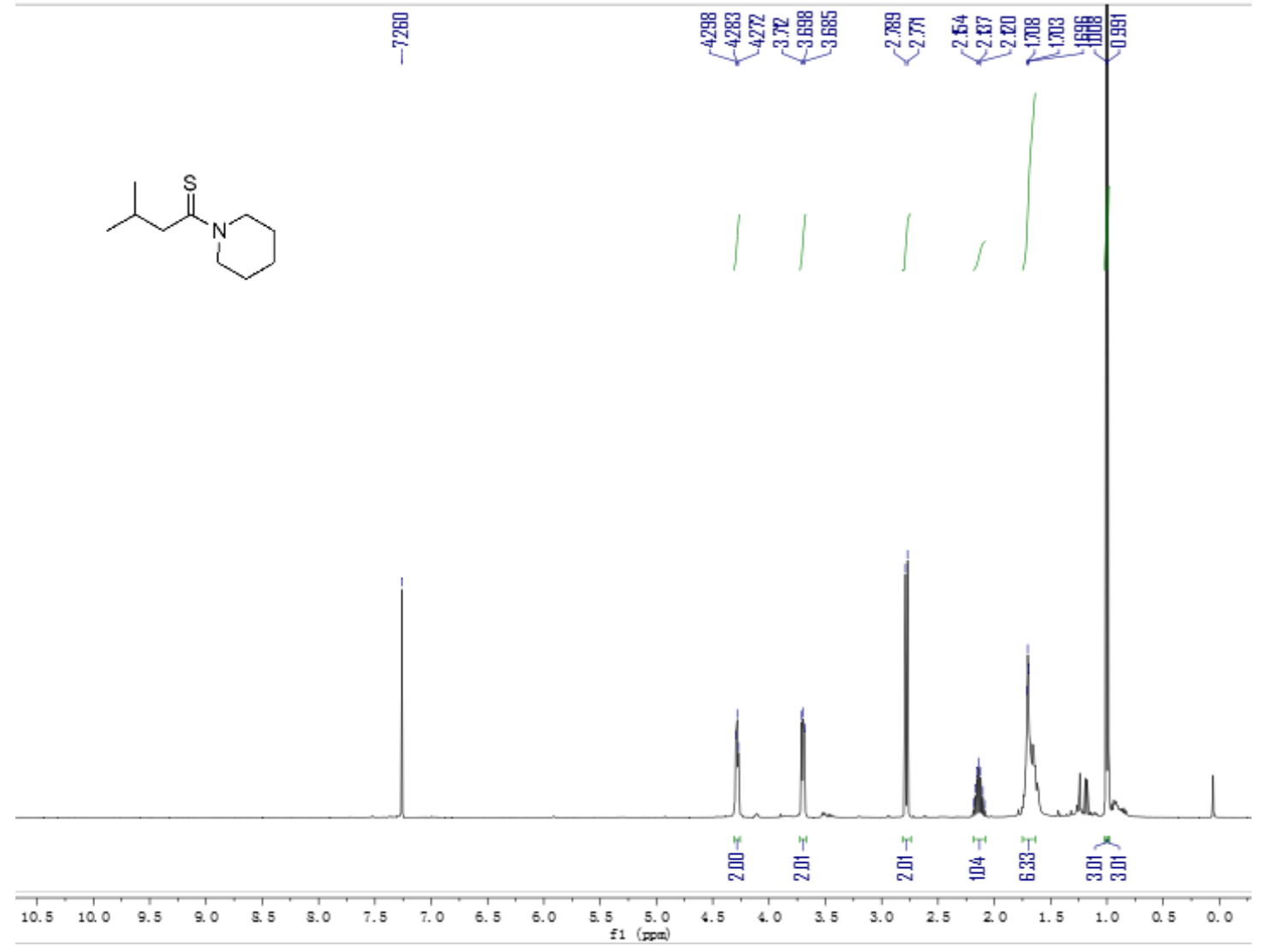

票

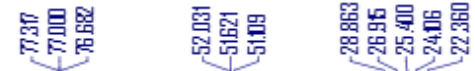
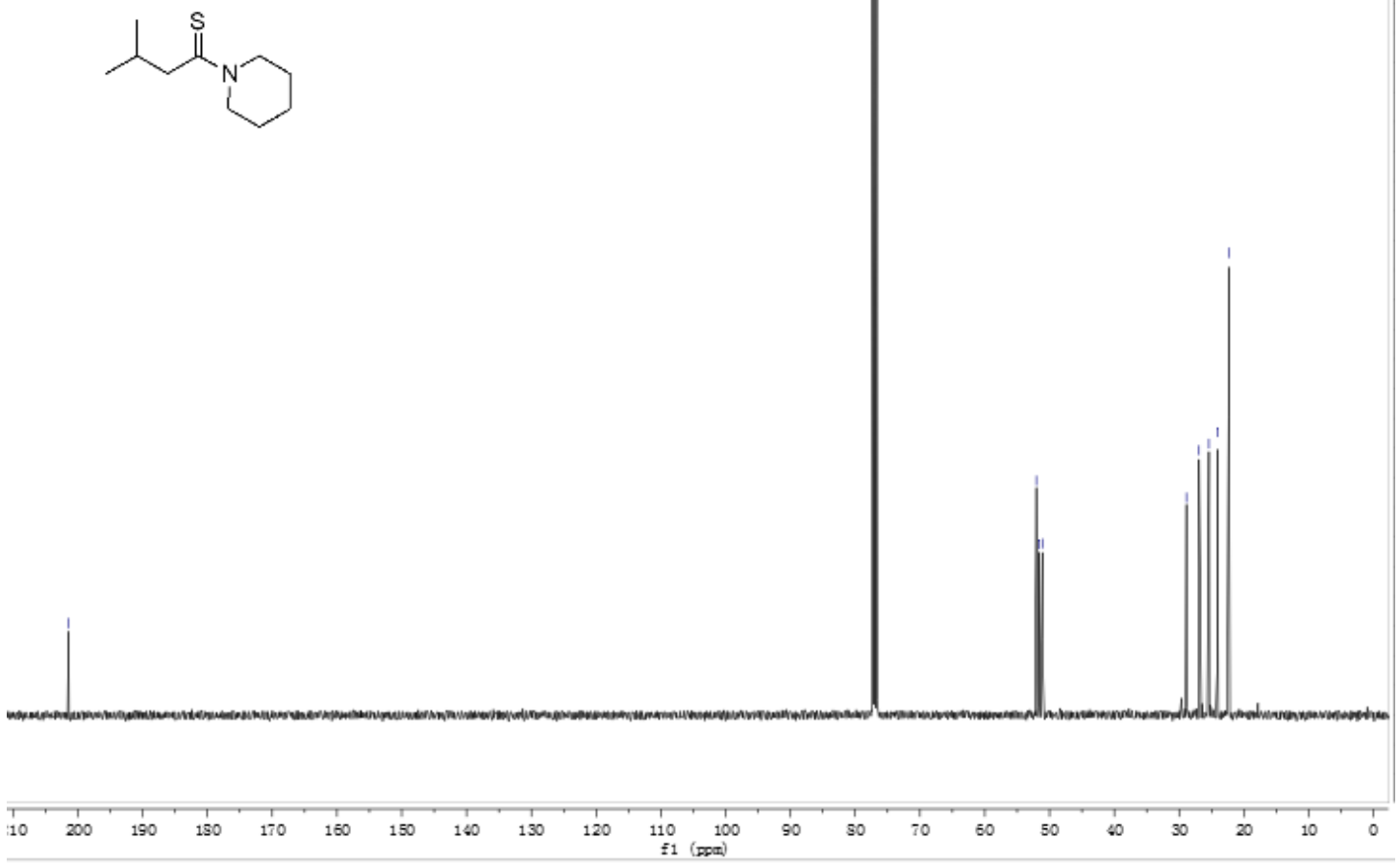

30 
2b:
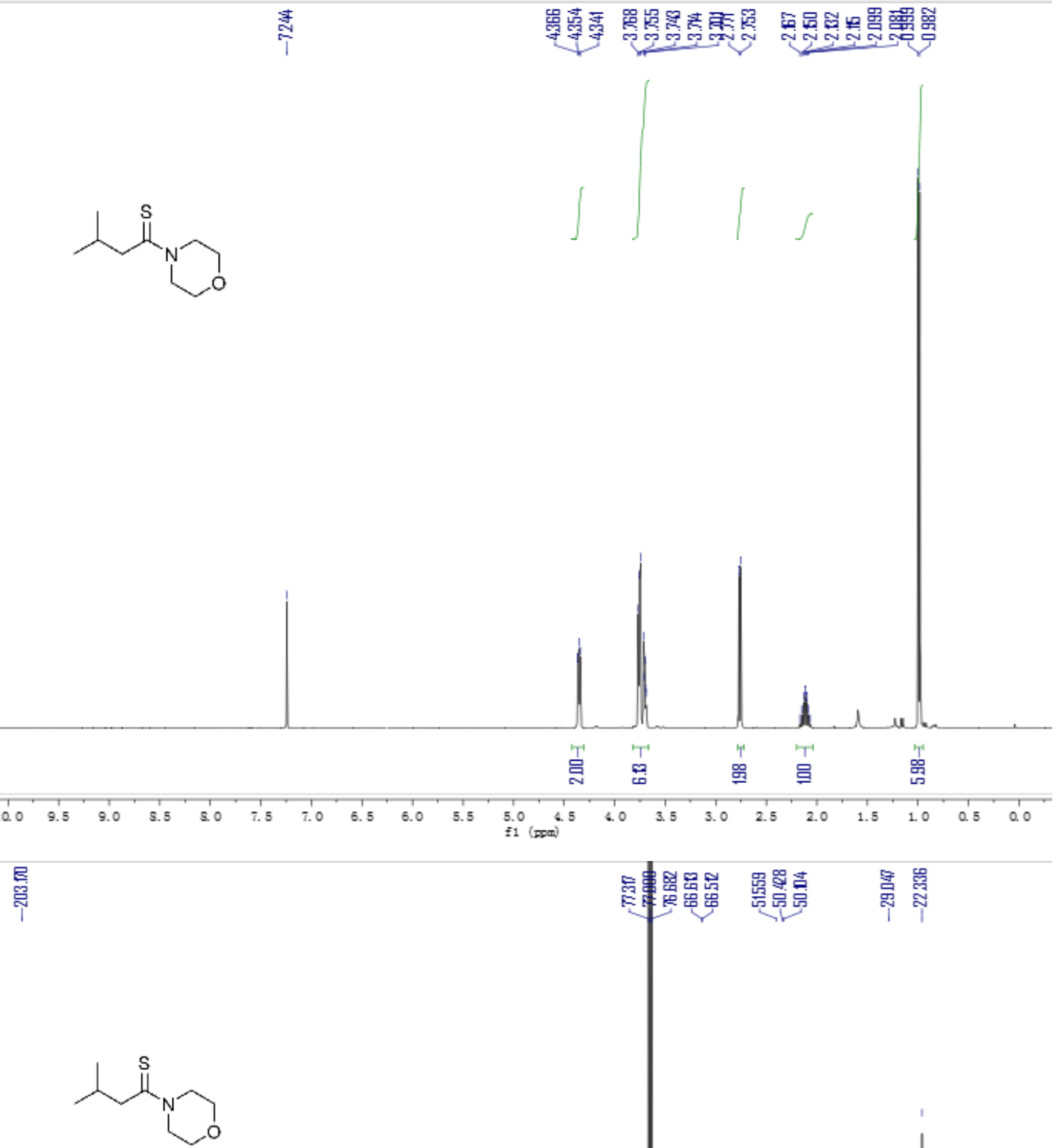

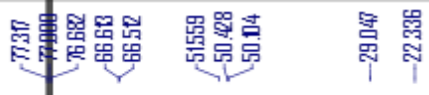

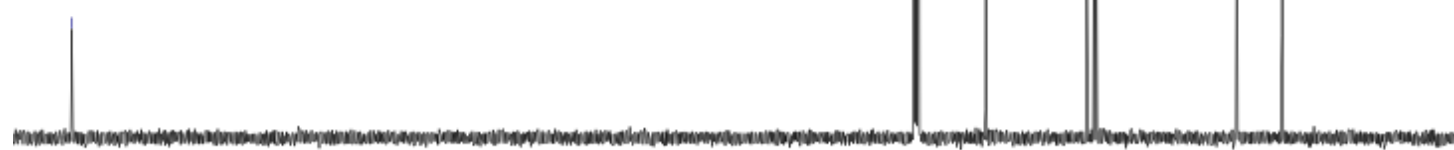

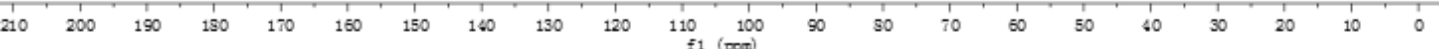


2c:
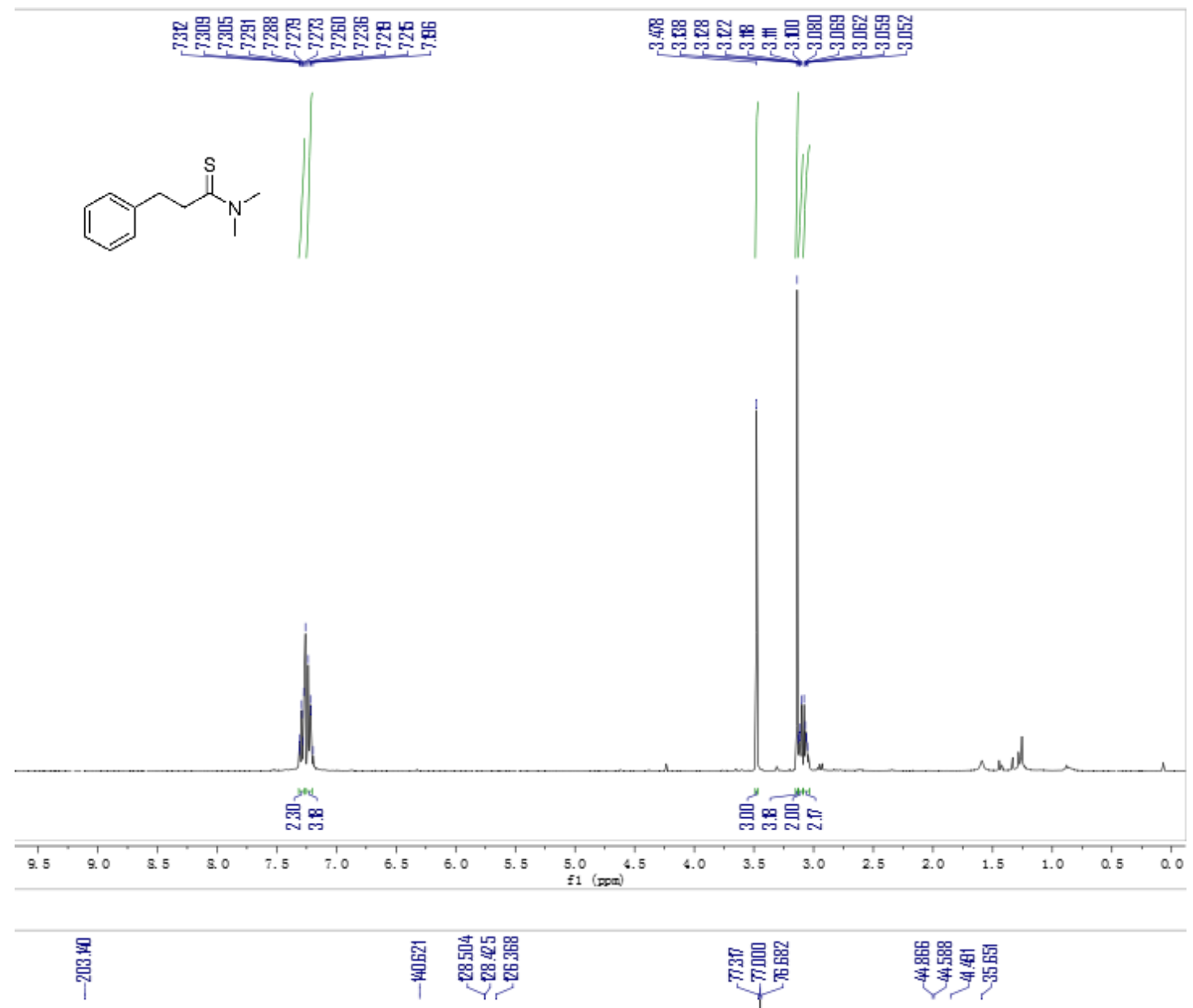

$\left(N_{N}^{\prime}\right.$

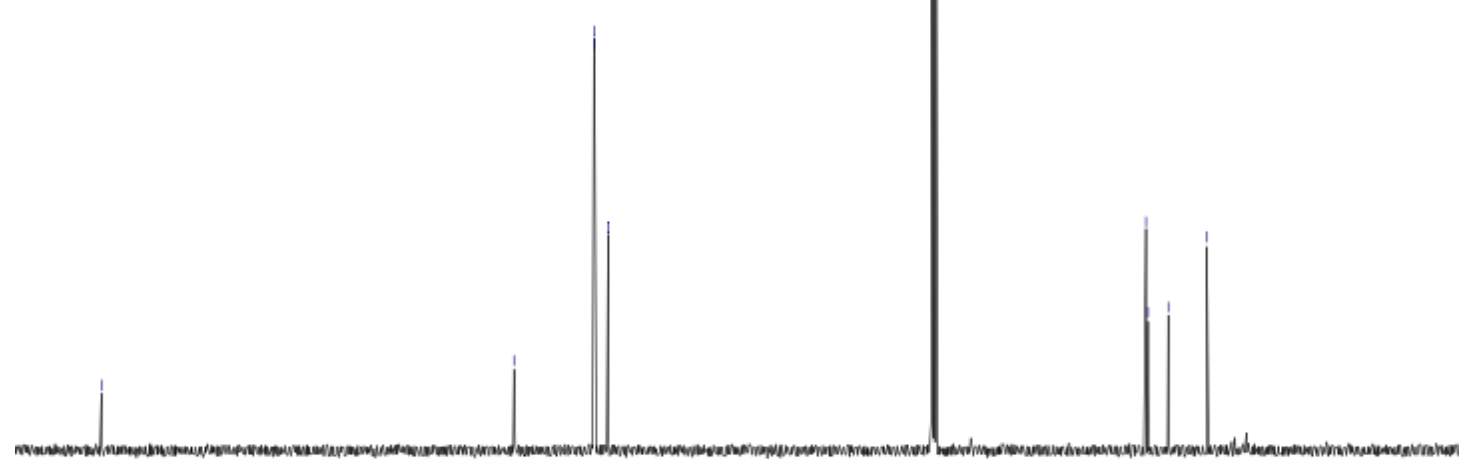

$\begin{array}{llllllllllllllllllllll}210 & 200 & 190 & 130 & 170 & 160 & 150 & 140 & 130 & 120 & \underset{f 1}{110} 100 & 90 & 50 & 70 & 60 & 50 & 40 & 30 & 20 & 10 & 0\end{array}$ 
2d:

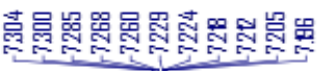

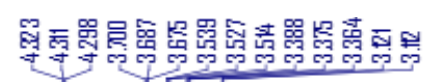
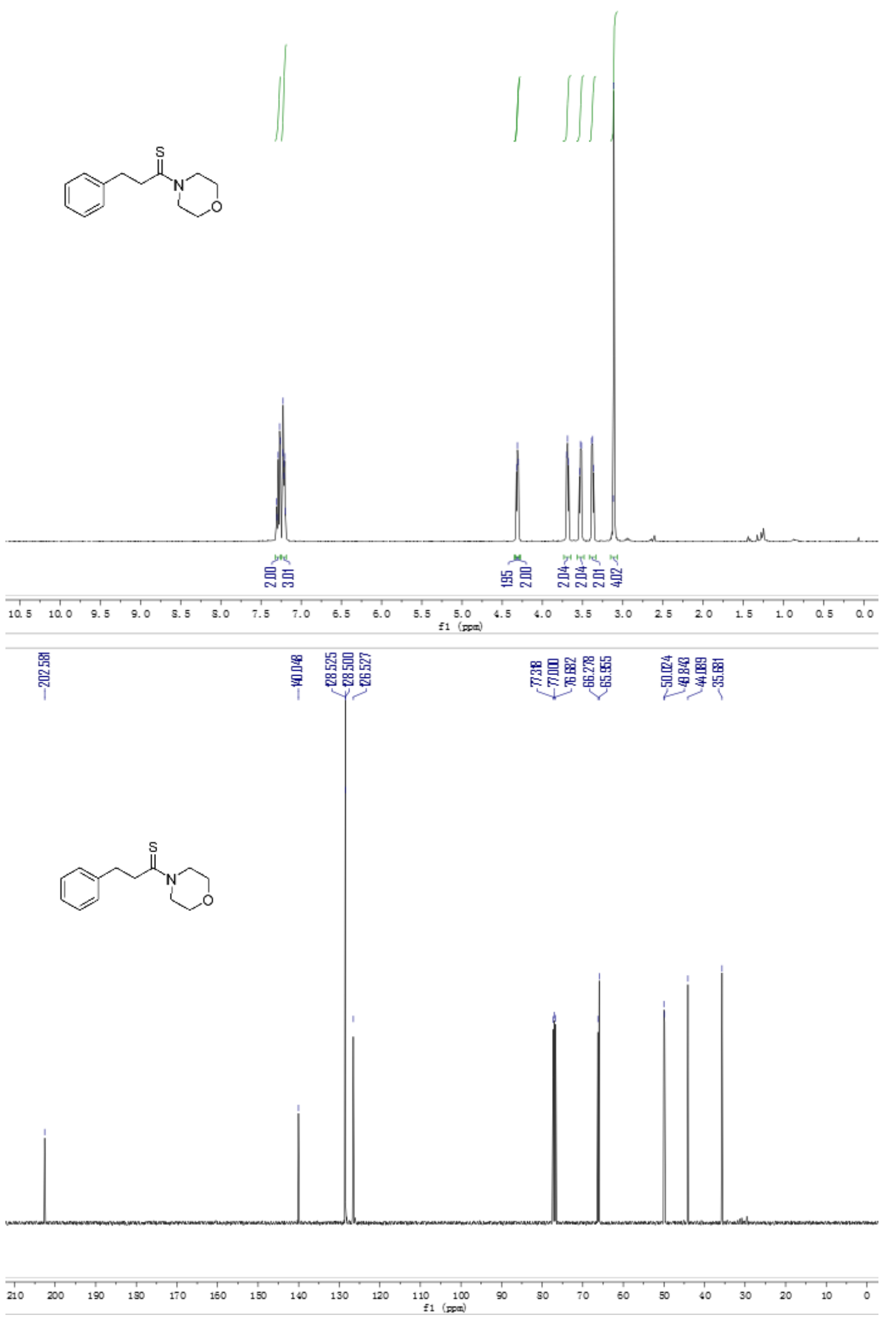

33 
2e:

足 品

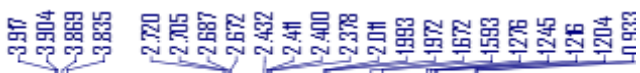
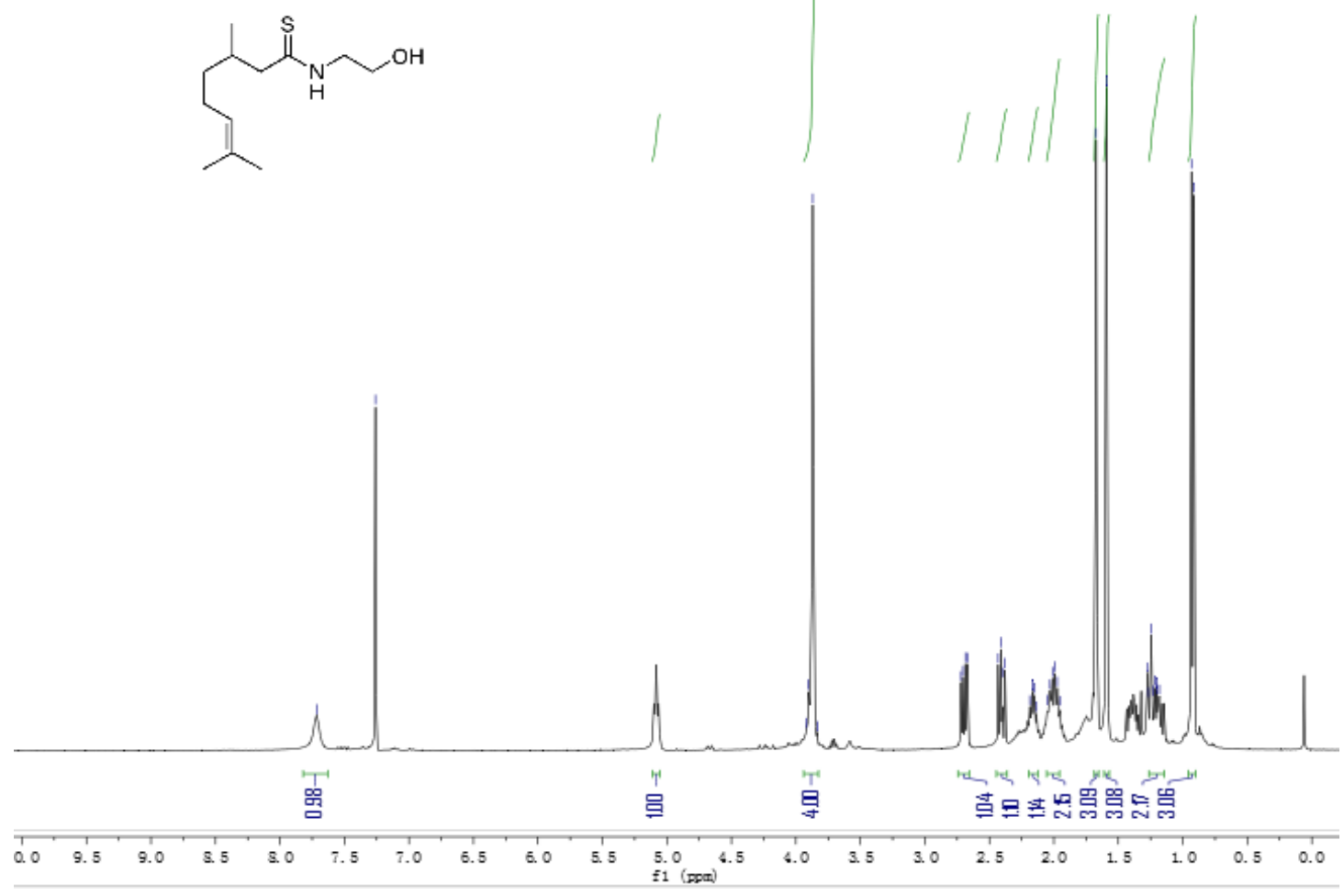

몸

兽昱

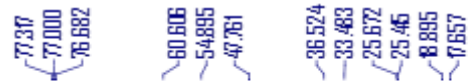<smiles>CC(C)=CCCC(C)CC(=S)NCCO</smiles>

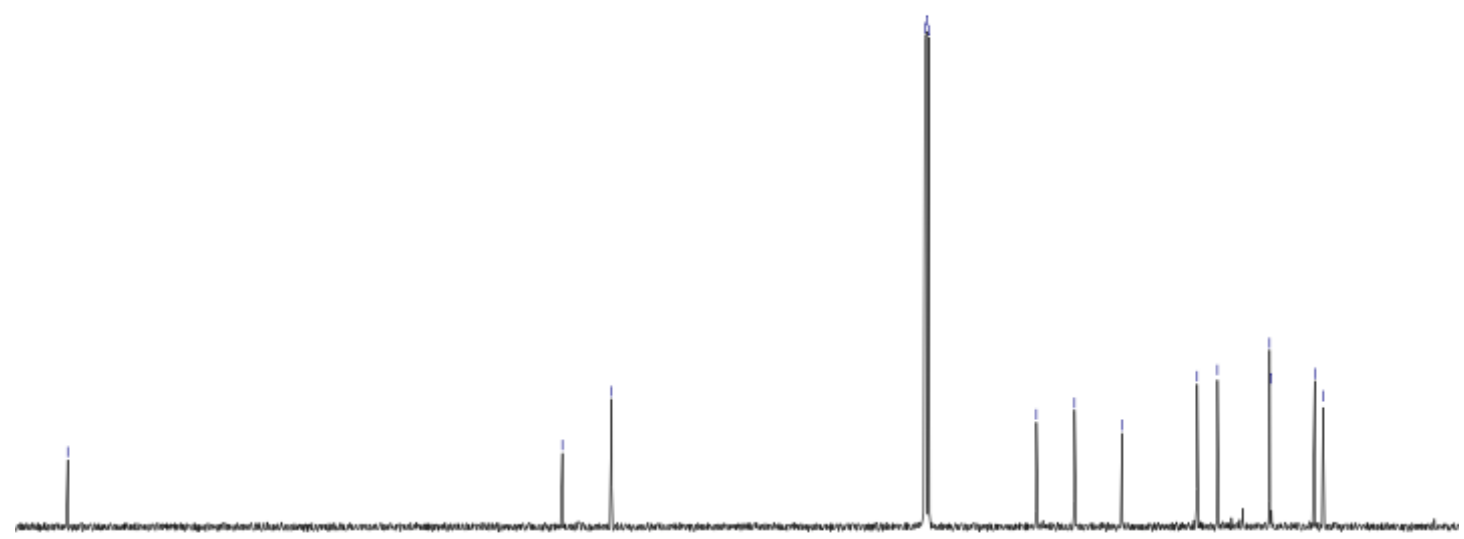

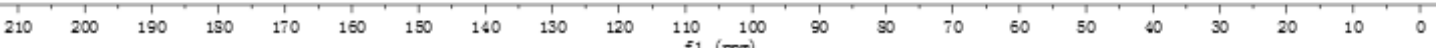


2f:

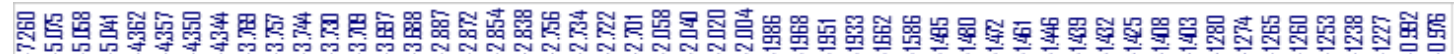<smiles>CC(C)=CCC(C)CC(=S)N1CCOCC1</smiles>

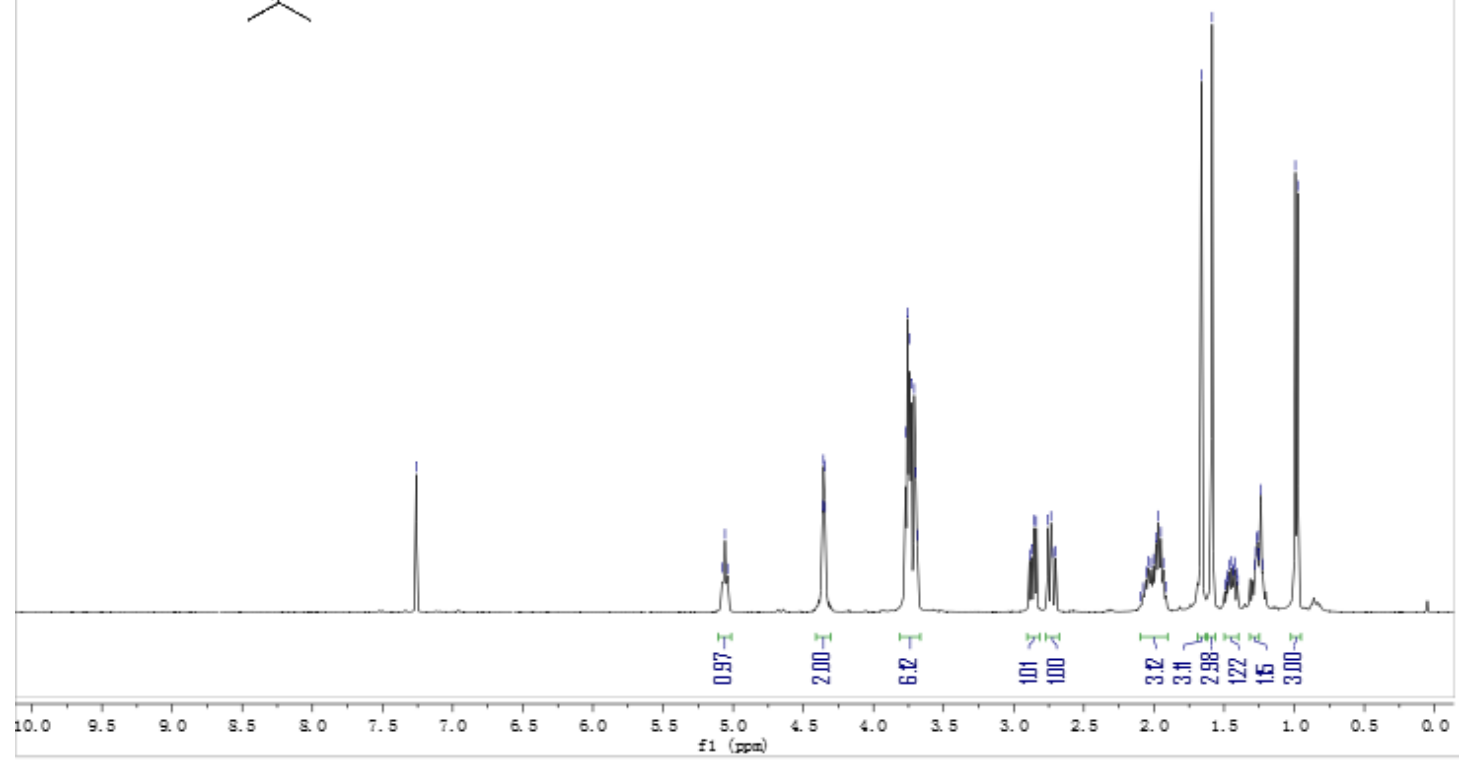

笣

罯要

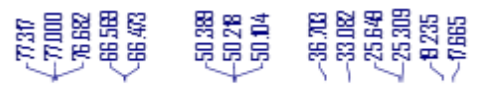<smiles>CC(C)=CCCC(C)CC(=S)N1CCOCC1</smiles>

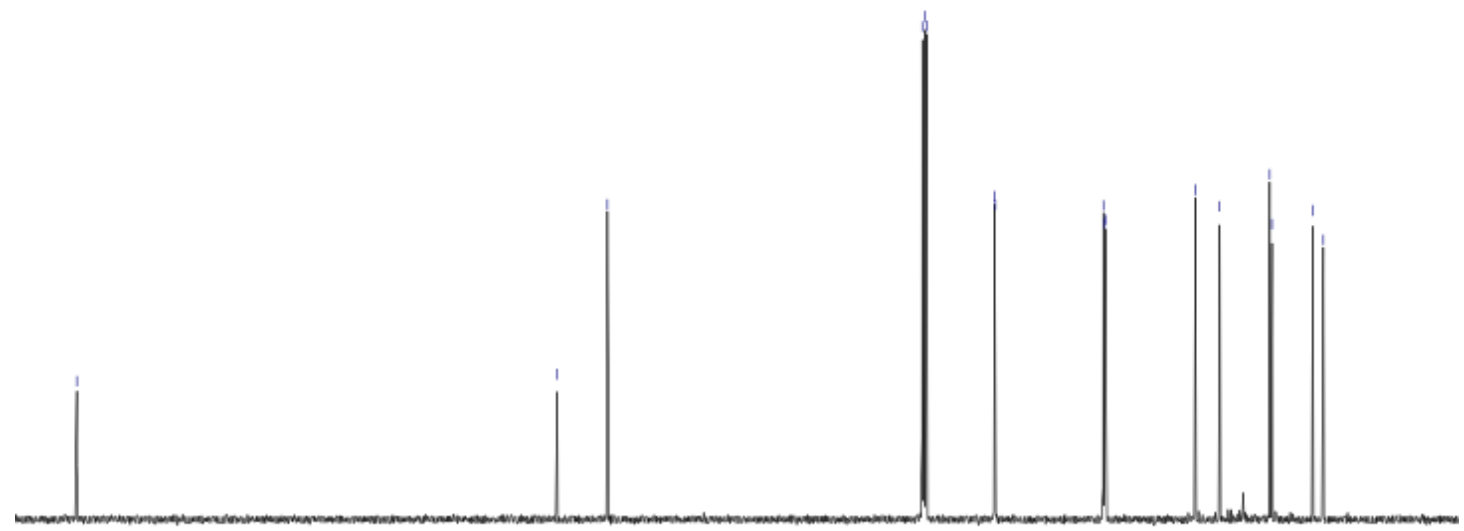

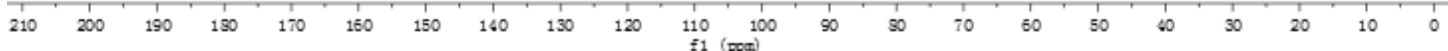


2g:

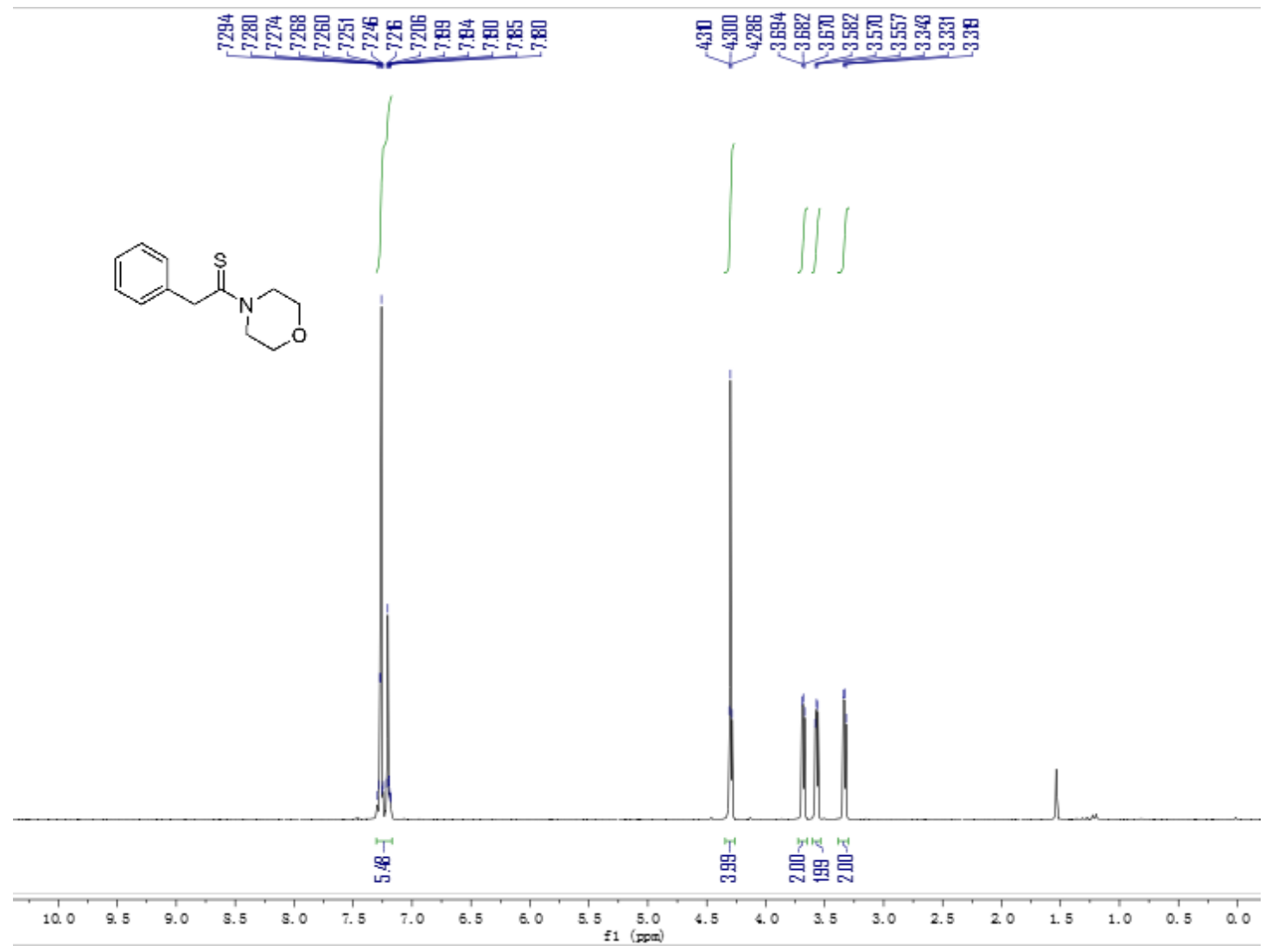

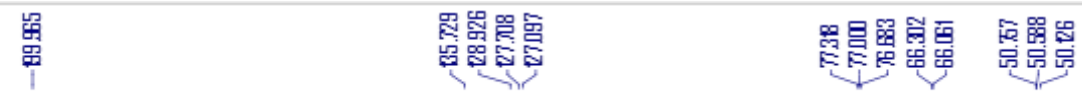

(1) 篮

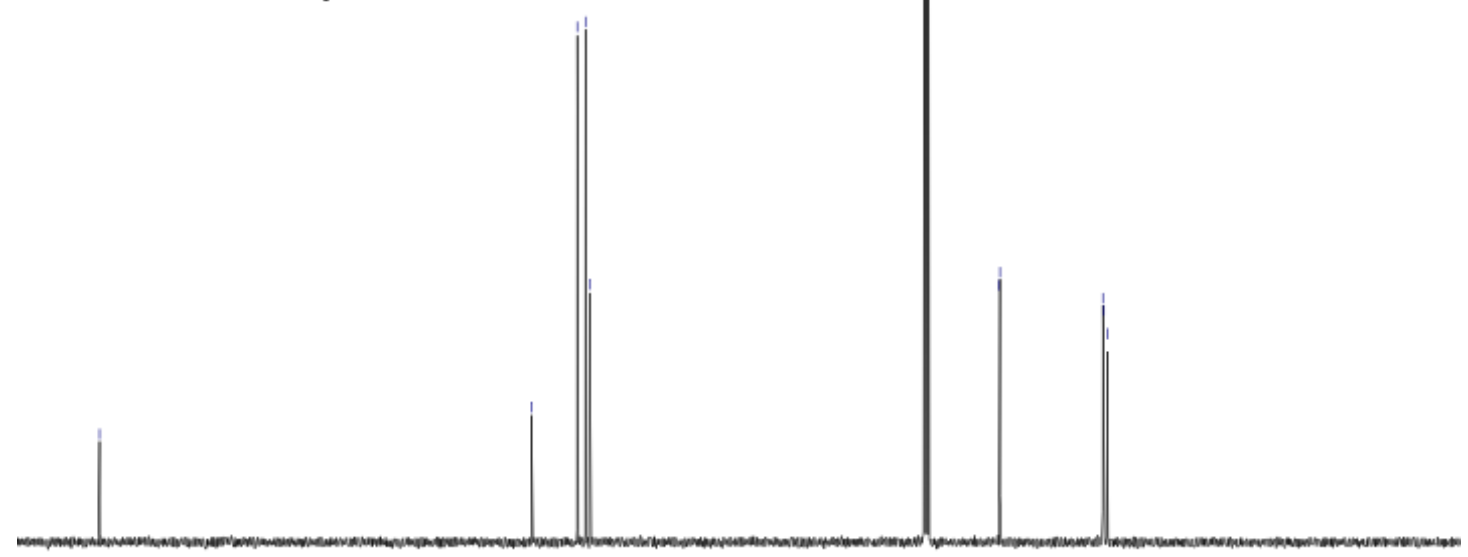

\begin{tabular}{llllllllllllllllllllllllll}
\hline 10 & 200 & 190 & 190 & 170 & 160 & 150 & 140 & 130 & 120 & 110 & 100 & 90 & 50 & 70 & 60 & 50 & 40 & 30 & 20 & 10 & 0
\end{tabular} 
2h:

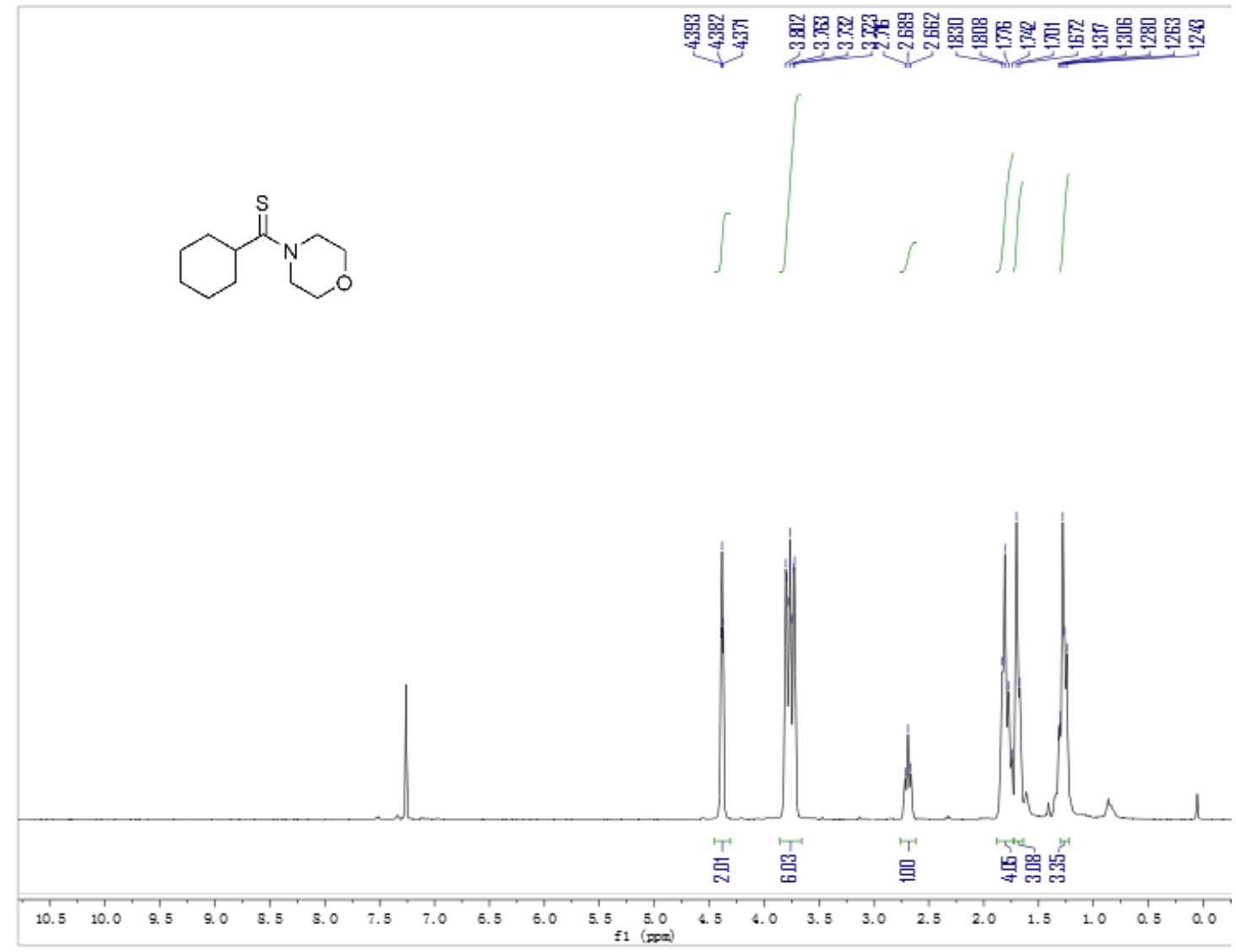

幡

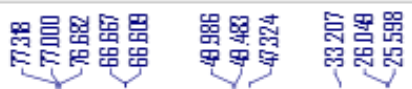

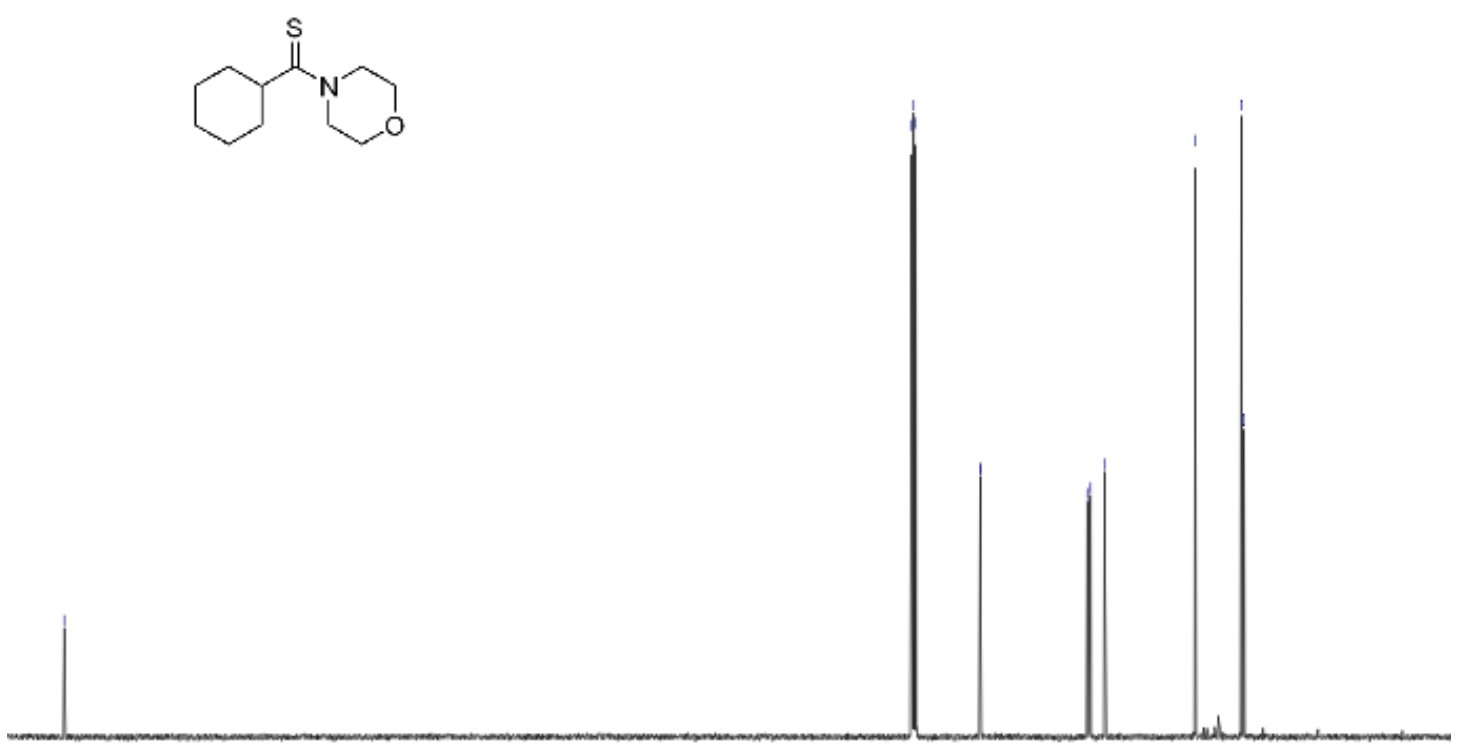

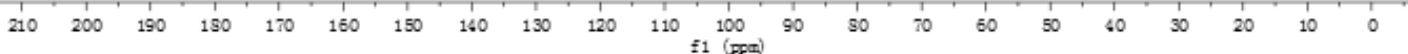


2i:

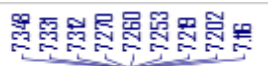

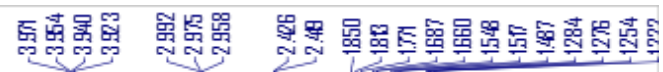

(C)
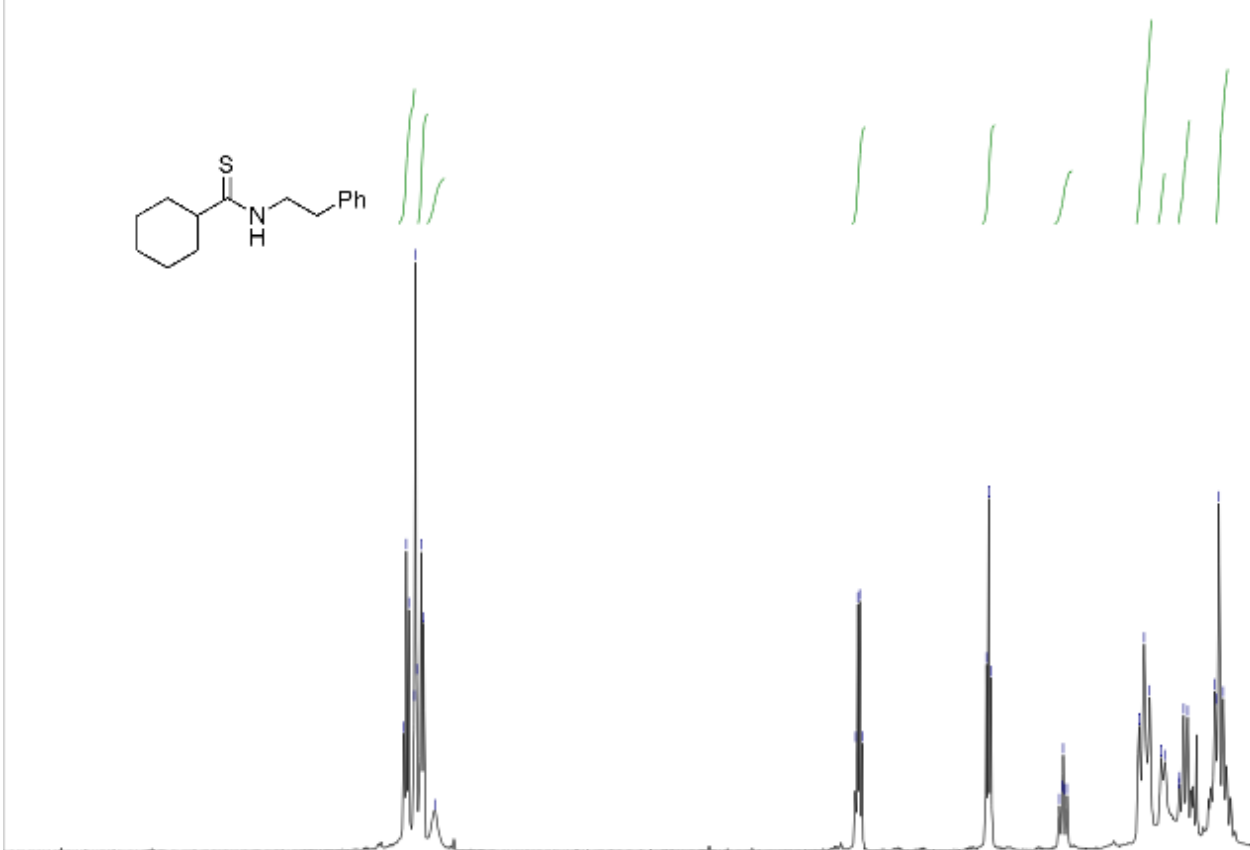

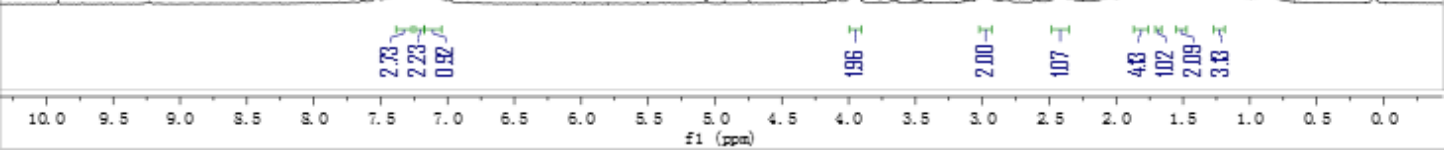

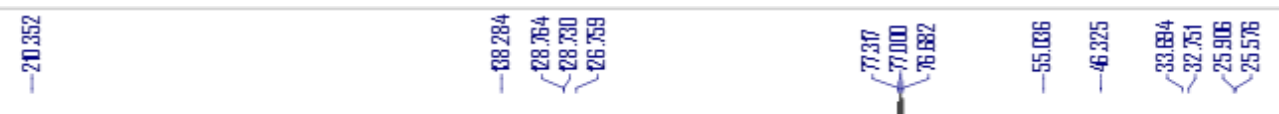

$\mathrm{C}_{\mathrm{H}}^{\mathrm{S}} \mathrm{\sim}^{\mathrm{Ph}}$

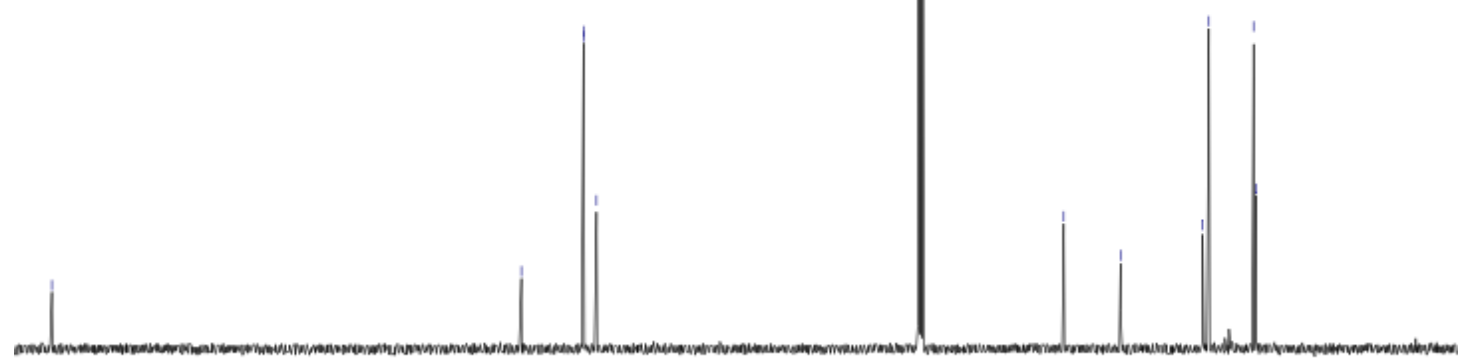

$210 \quad 200 \quad 190 \quad 190 \quad 170 \quad 160 \quad 150 \quad 140 \quad 130 \quad 120 \quad \underset{f 1}{110} 10$ 
2j:

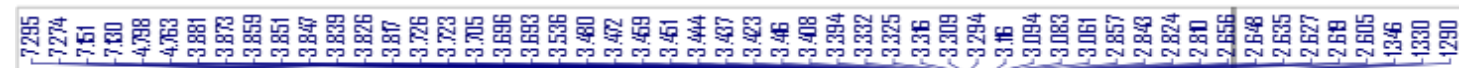

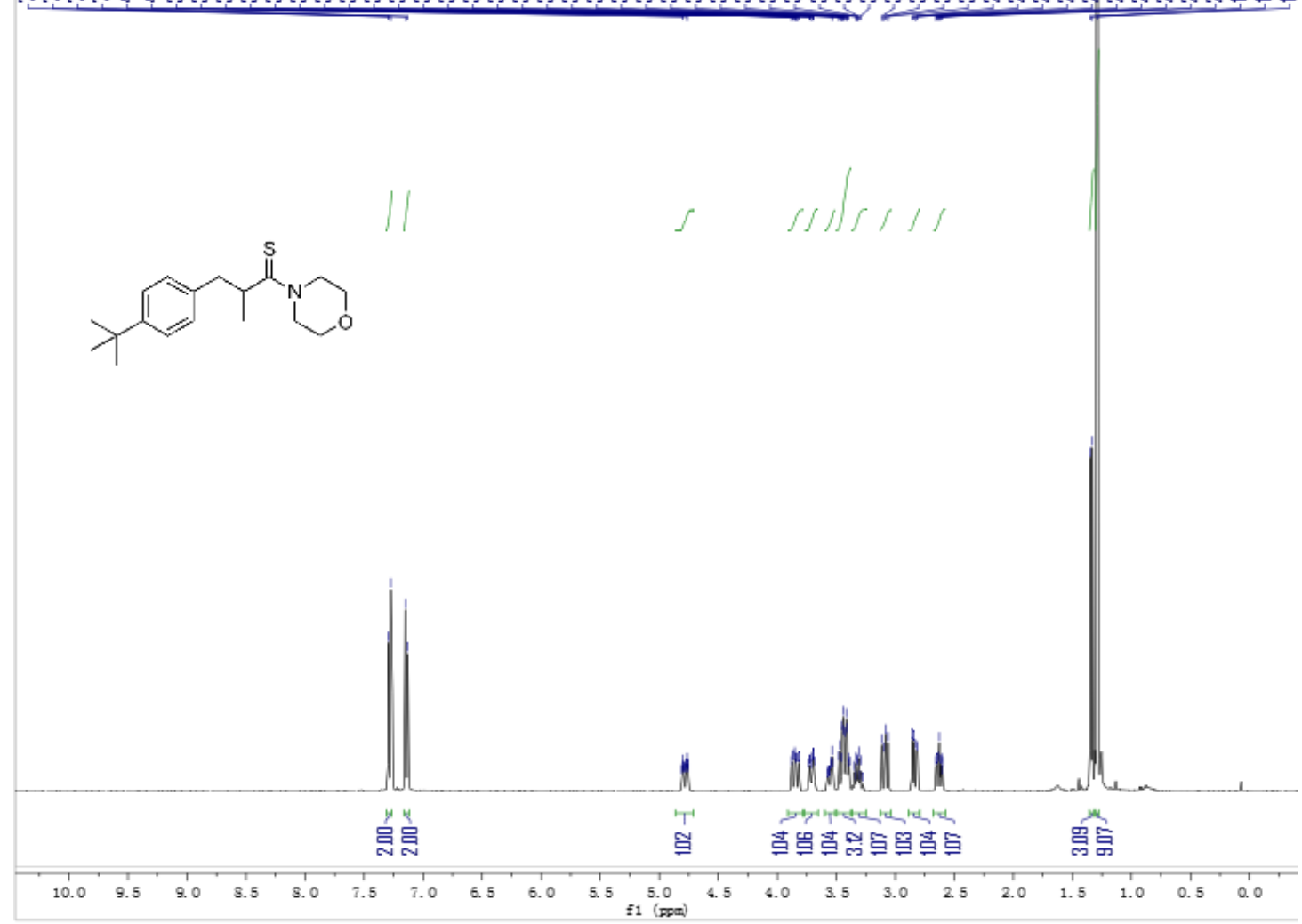

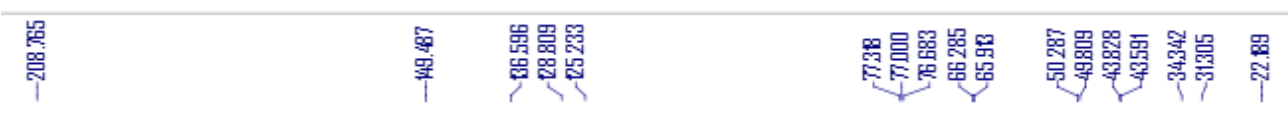
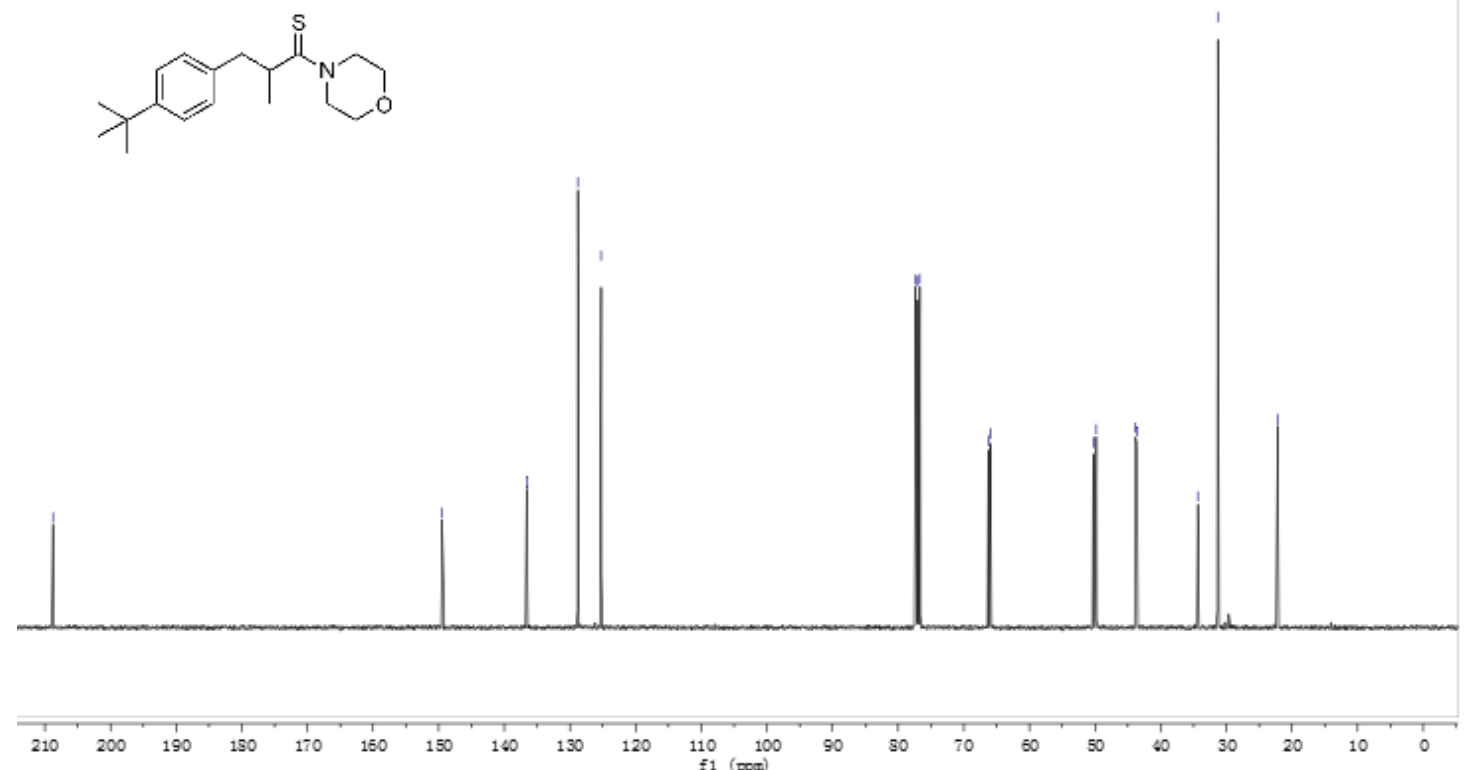
2k:

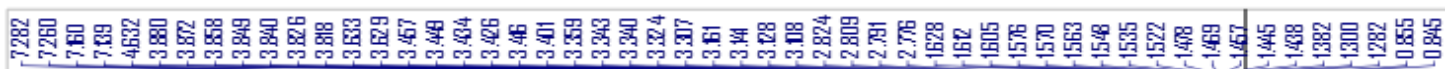
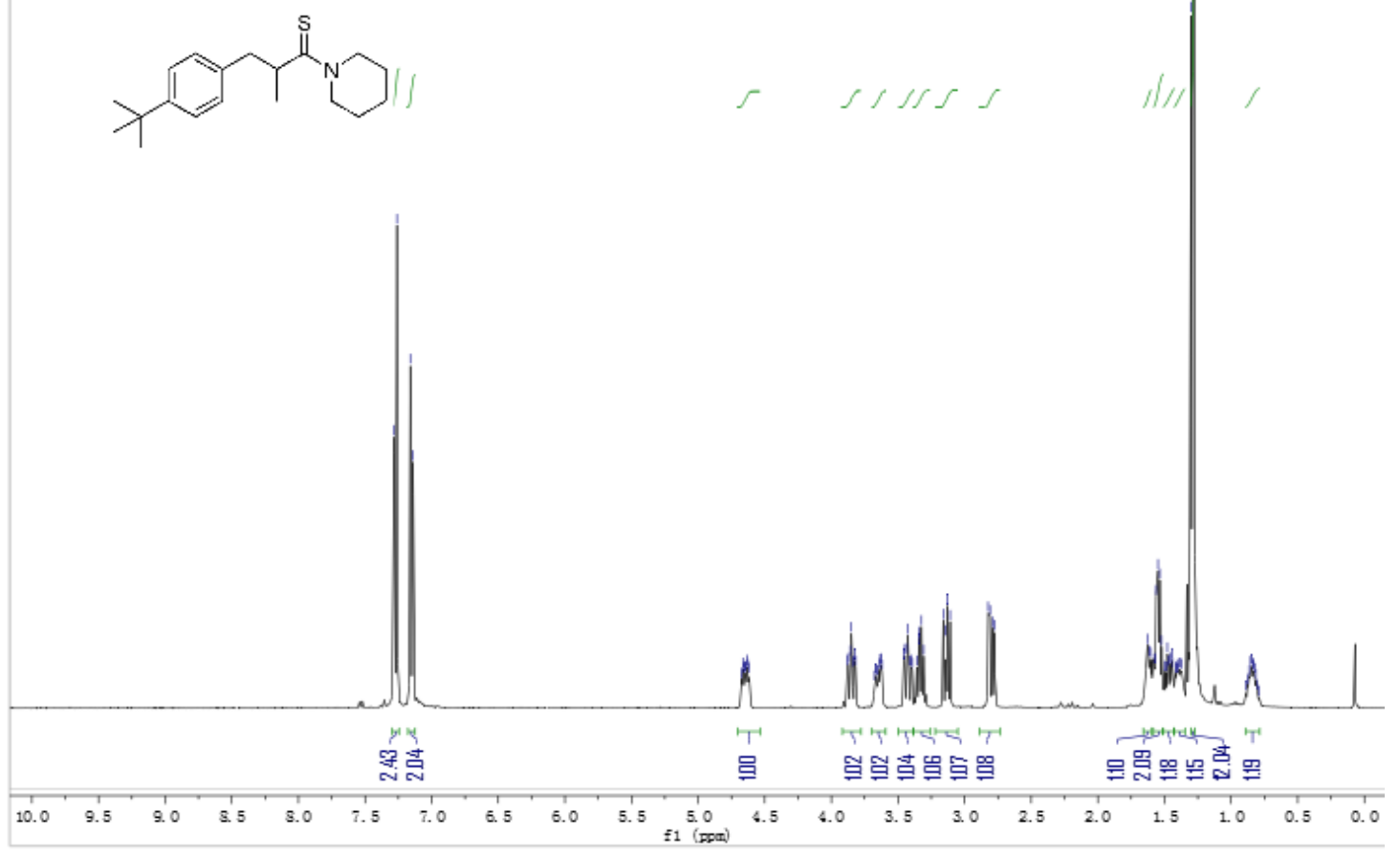

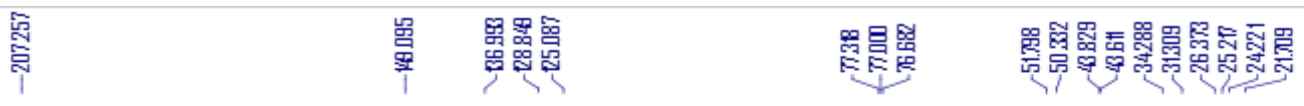
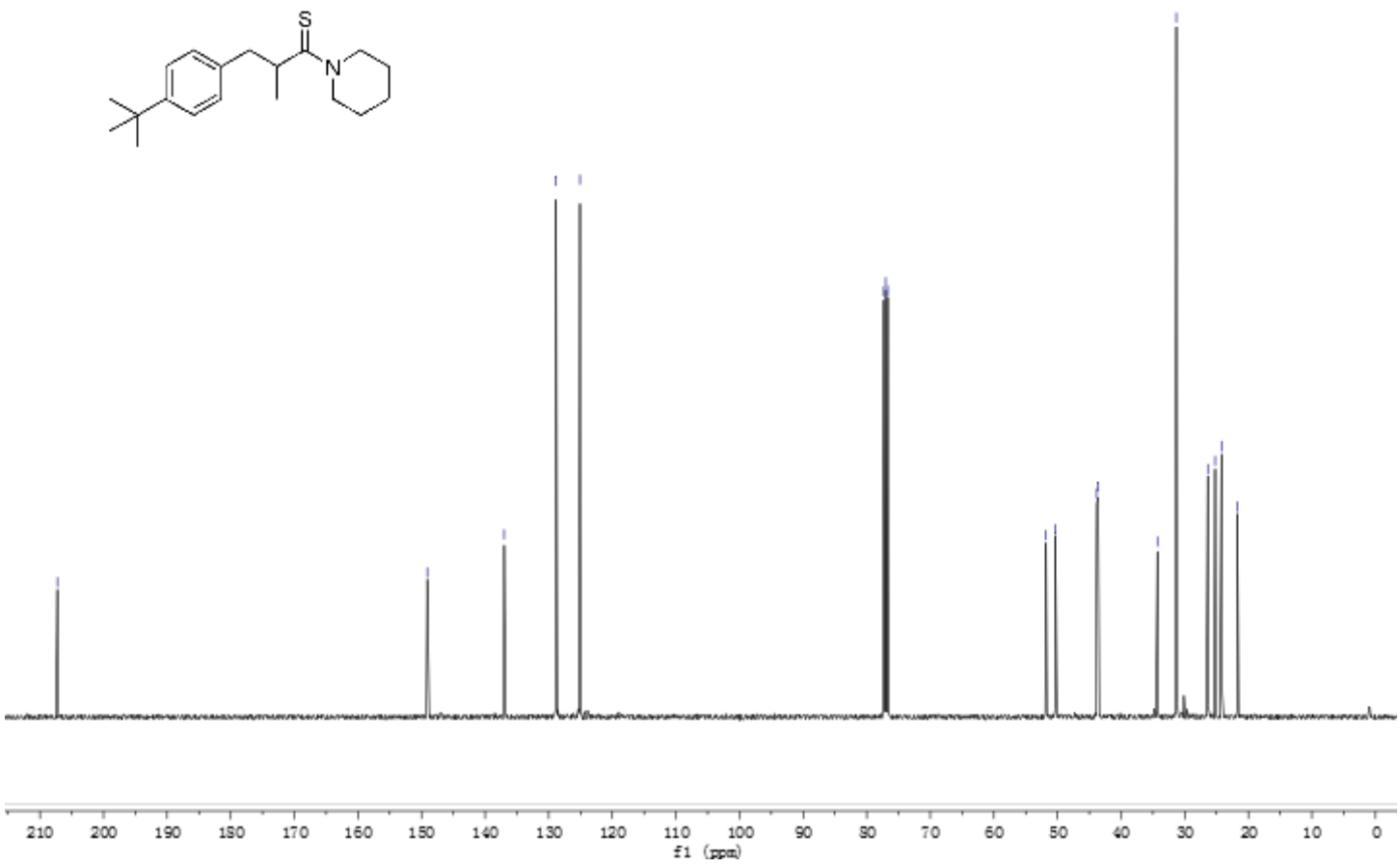

40 
21:

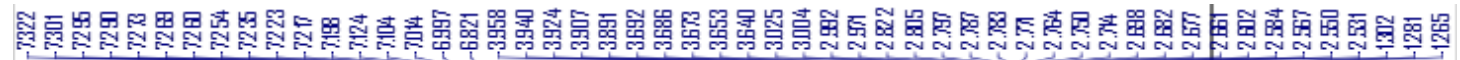
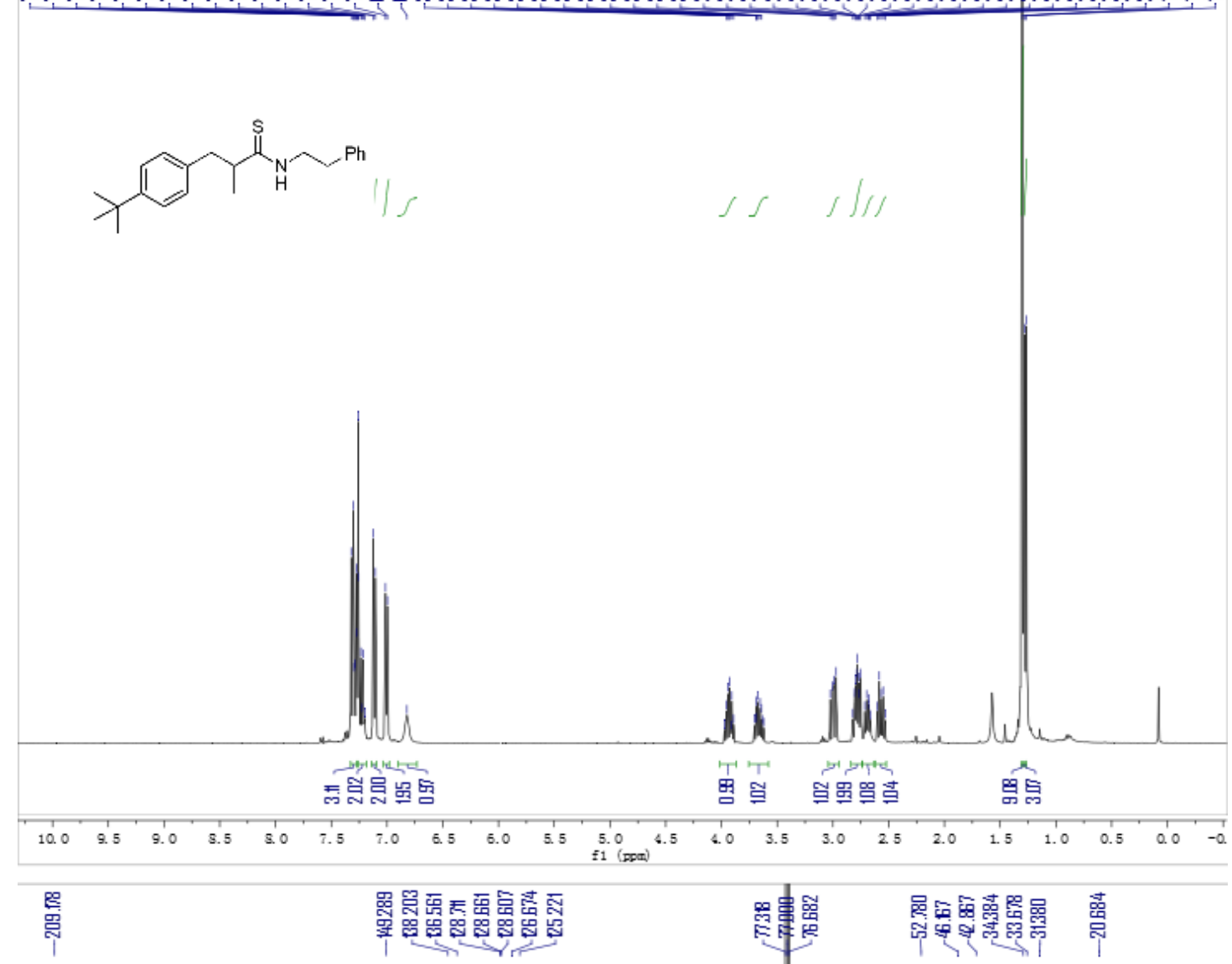

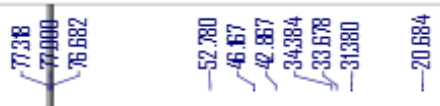<smiles>CC(Cc1ccc(C(C)(C)C)cc1)C(=O)NCCc1ccccc1</smiles>

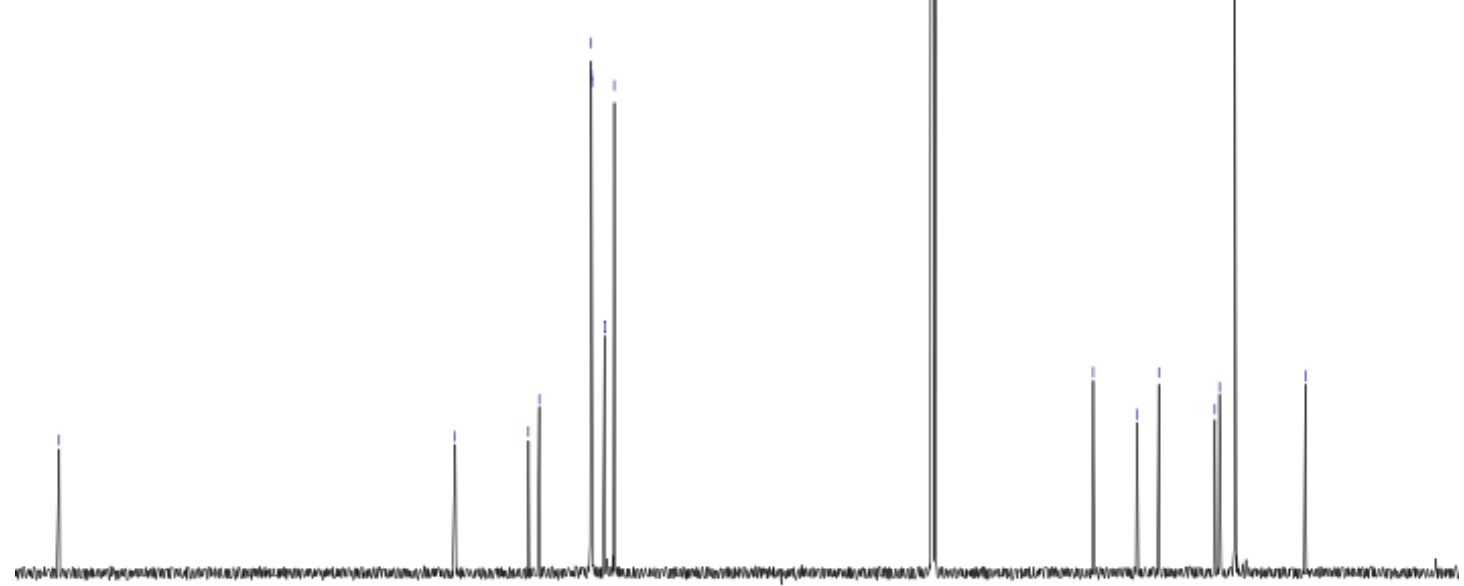

$\begin{array}{llllllllllll}210 & 200 & 190 & 190 & 170 & 160 & 150 & 140 & 130 & 120 & 110 & 100\end{array}$ 
2m:

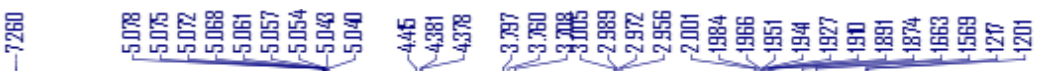
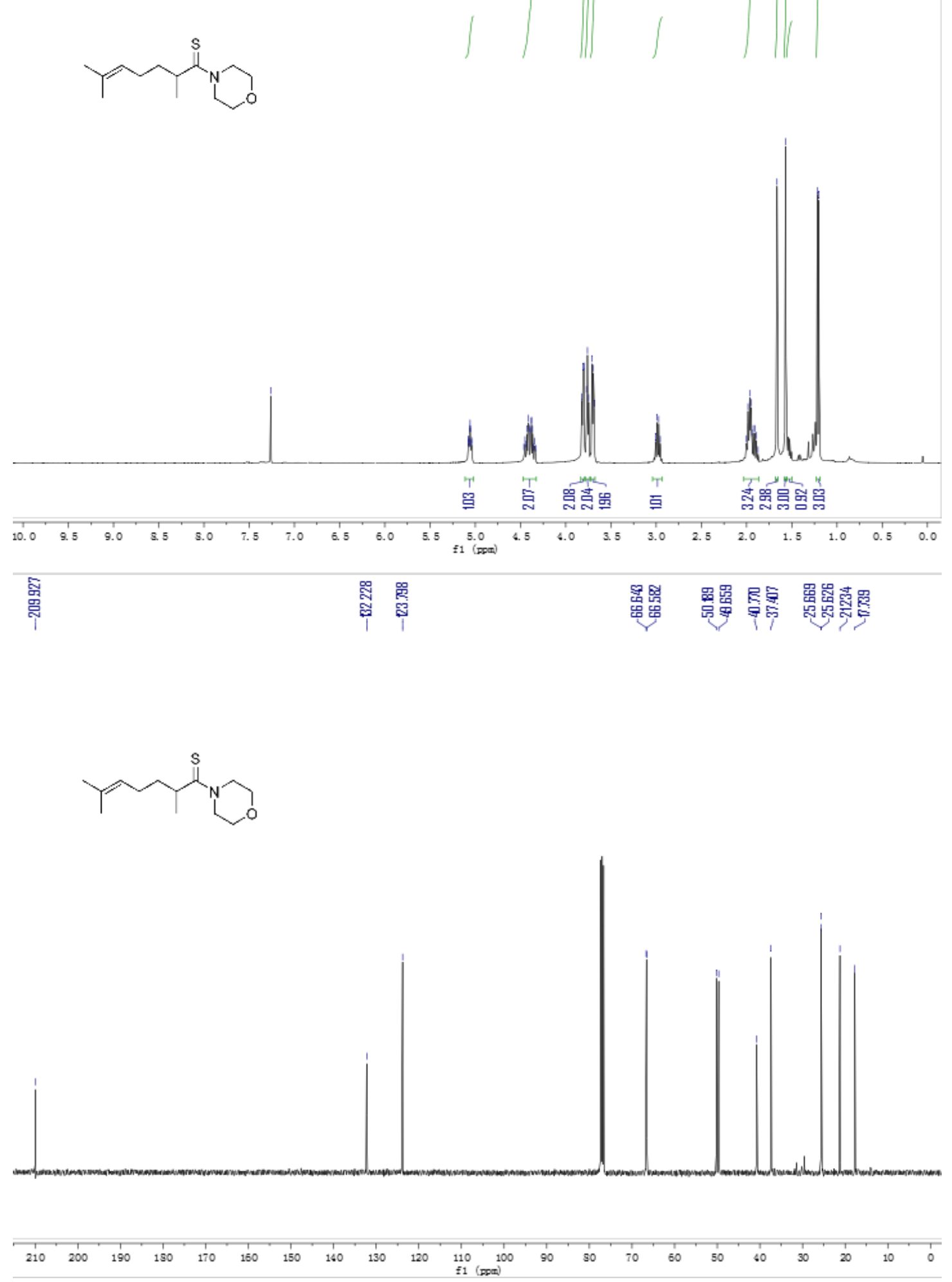

42 
SI-1 :

蜴

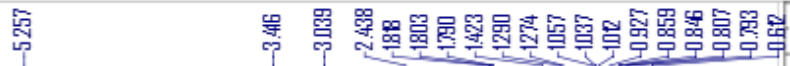
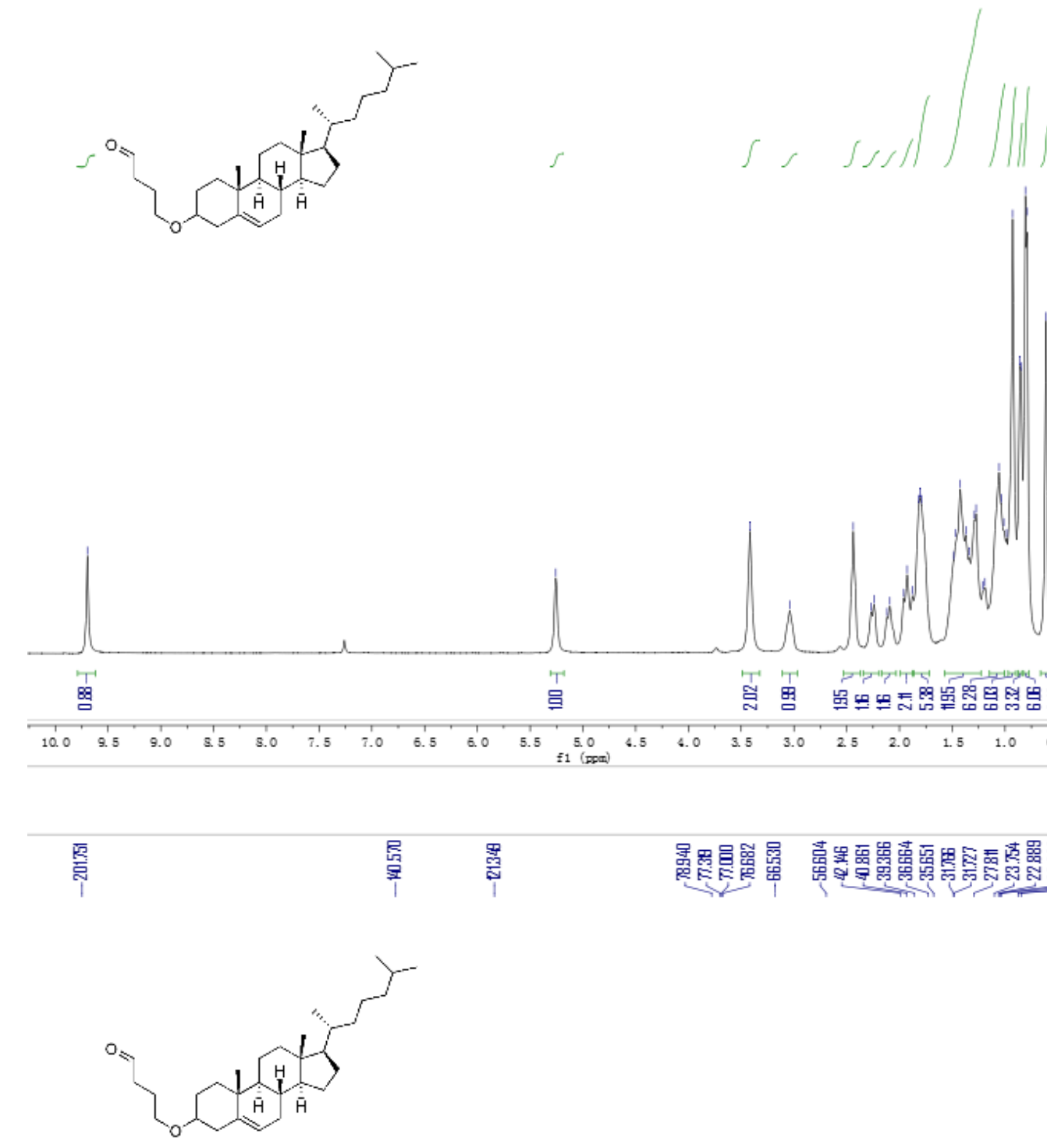
2n:

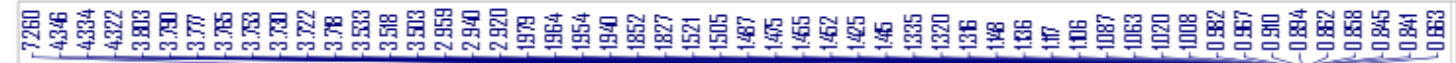
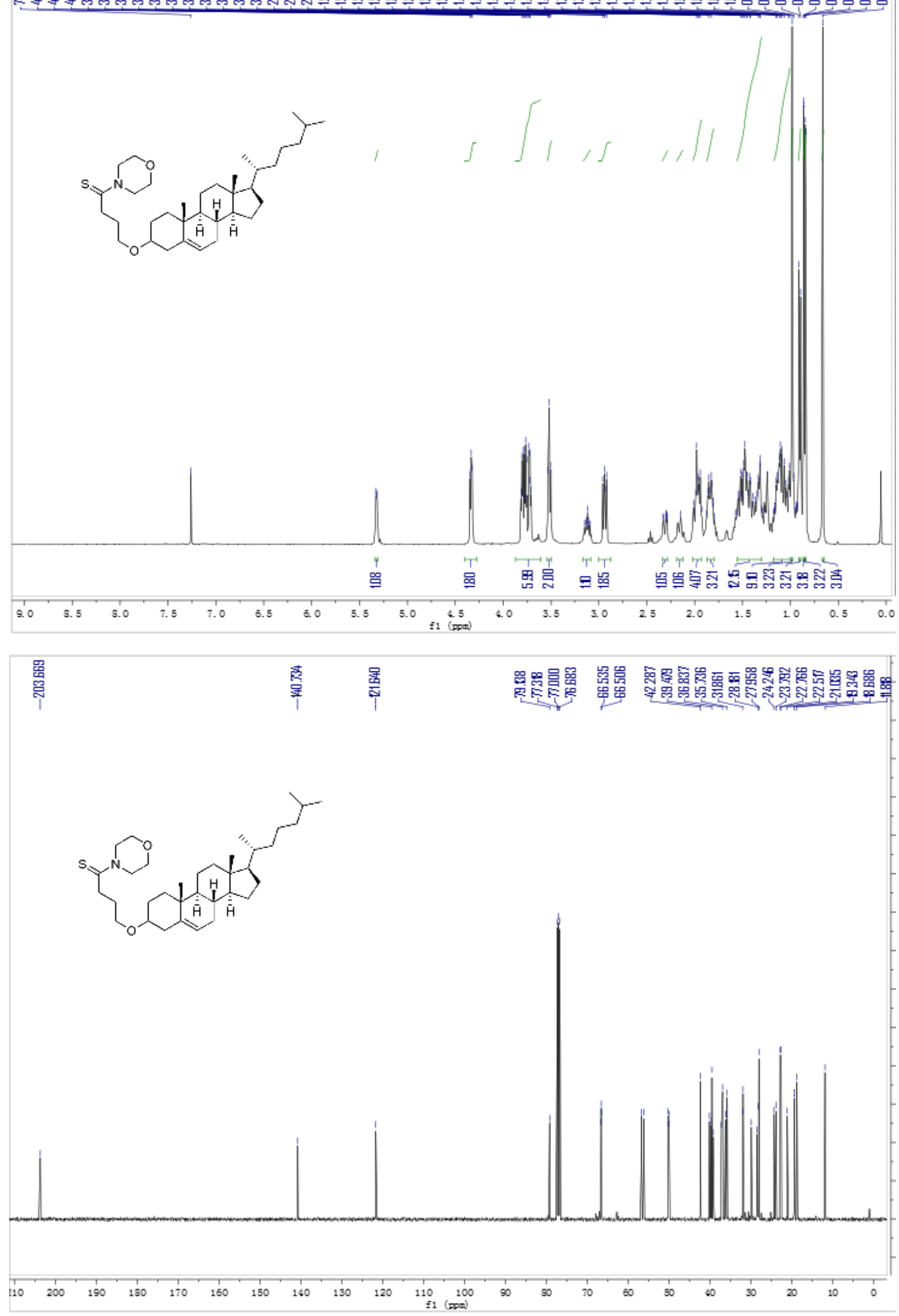

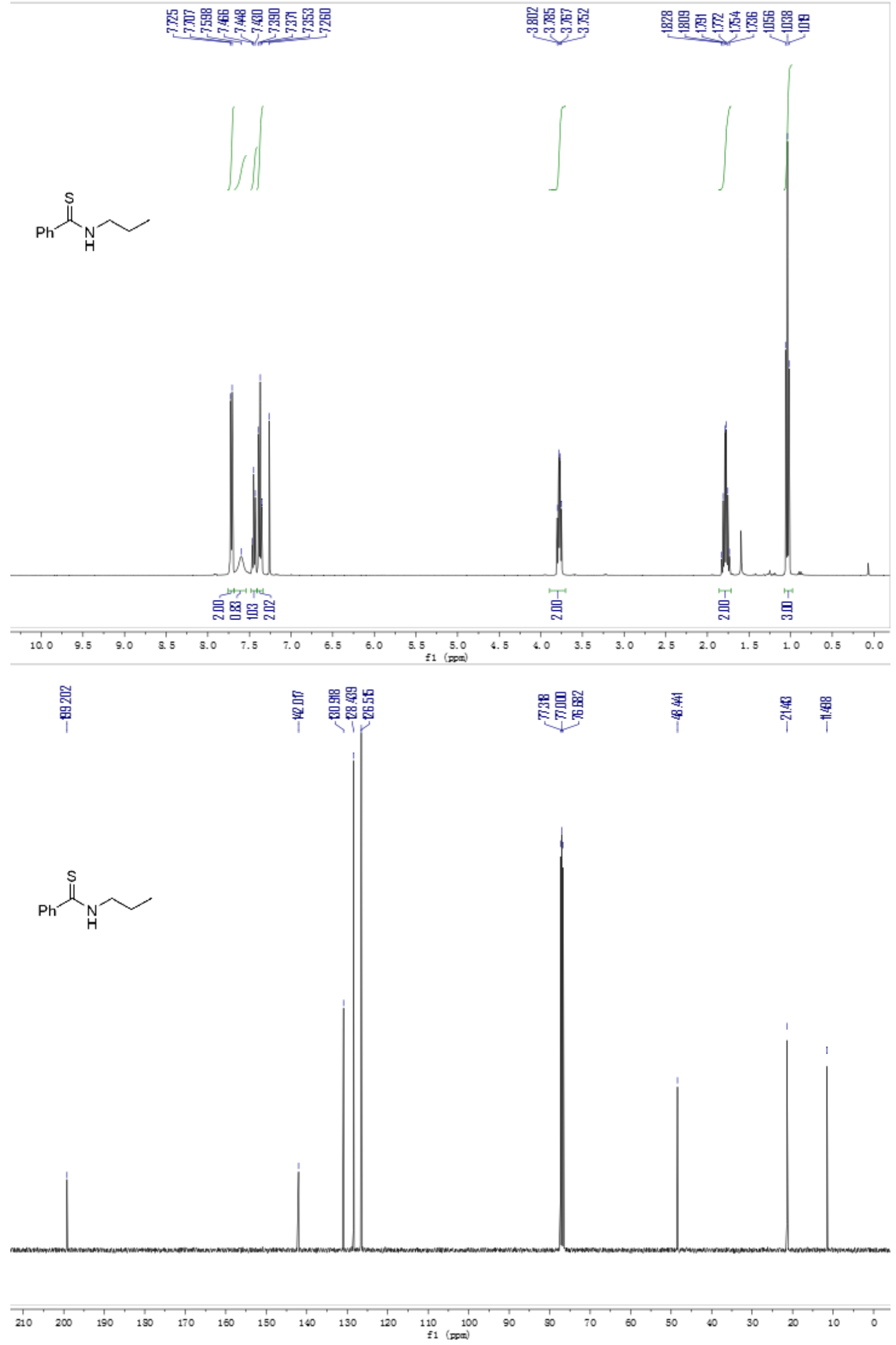
3b:

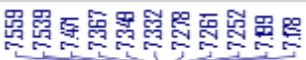

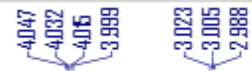
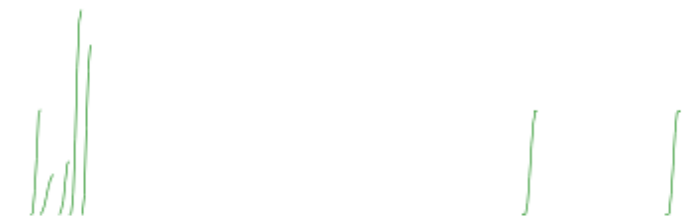

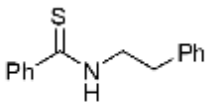
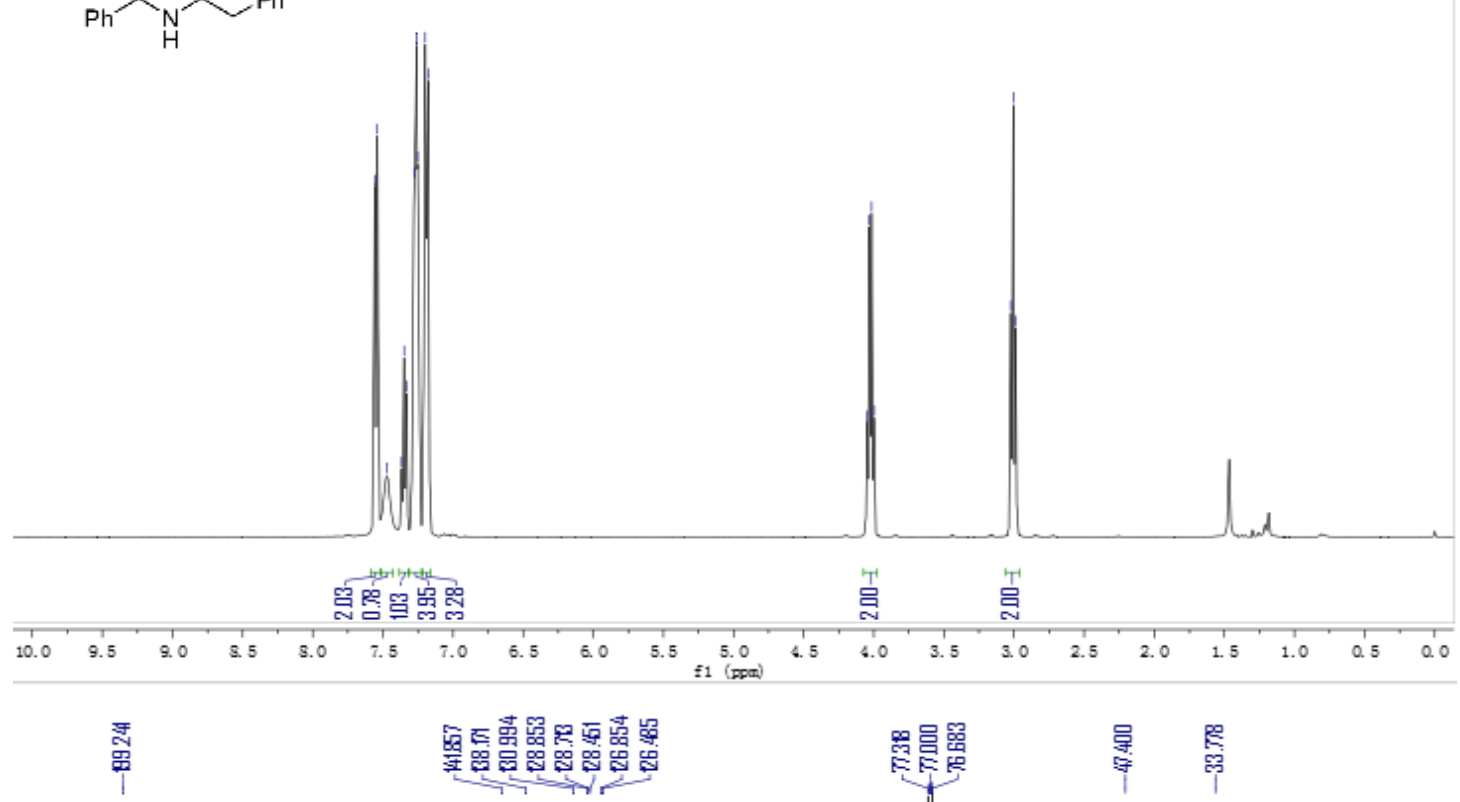

$\mathrm{Ph}_{\mathrm{H}}^{\mathrm{L}} \overbrace{\mathrm{Ph}}^{\mathrm{L}}$

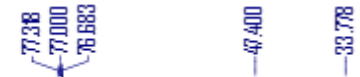

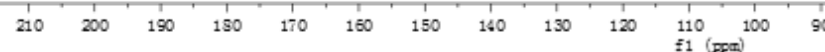


3c:

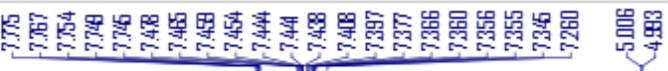

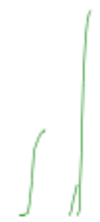

$\mathrm{Ph}_{\mathrm{H}}^{\mathrm{S}} \mathrm{Ph}$

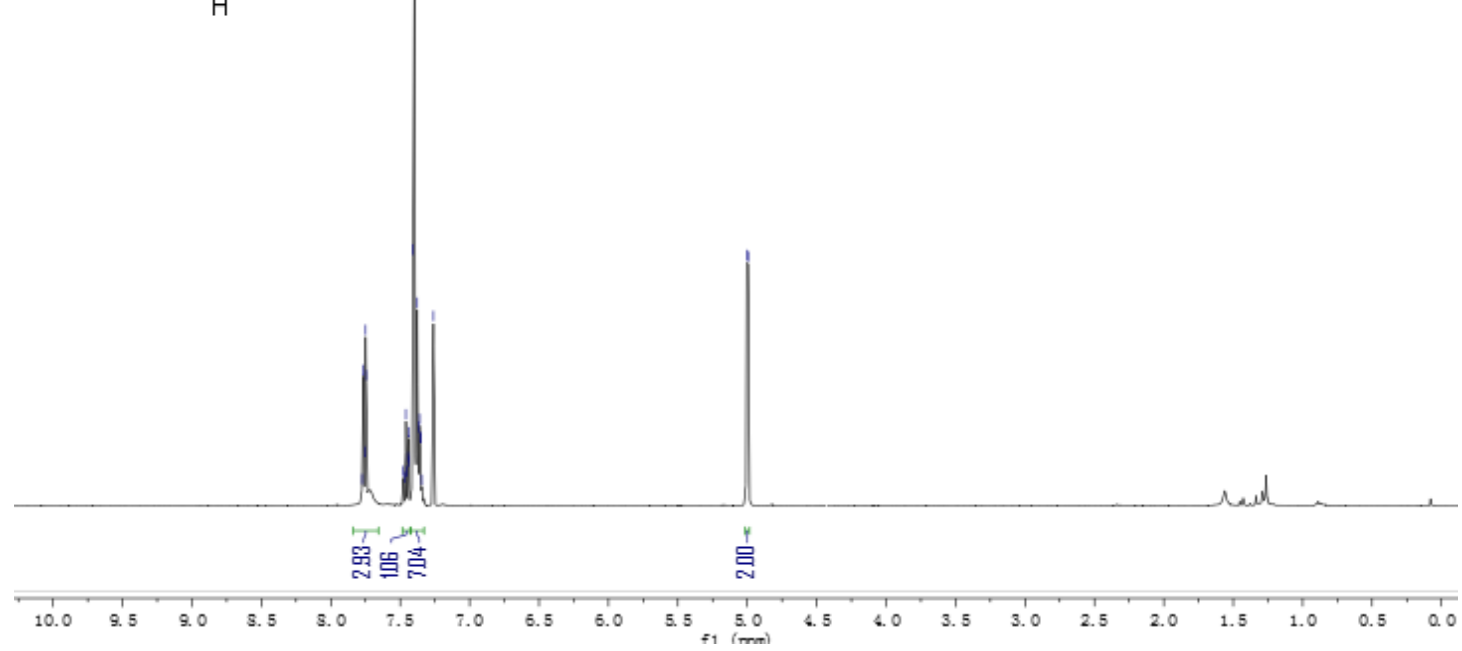

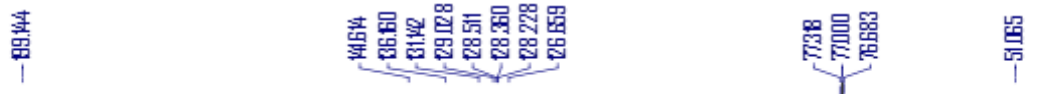

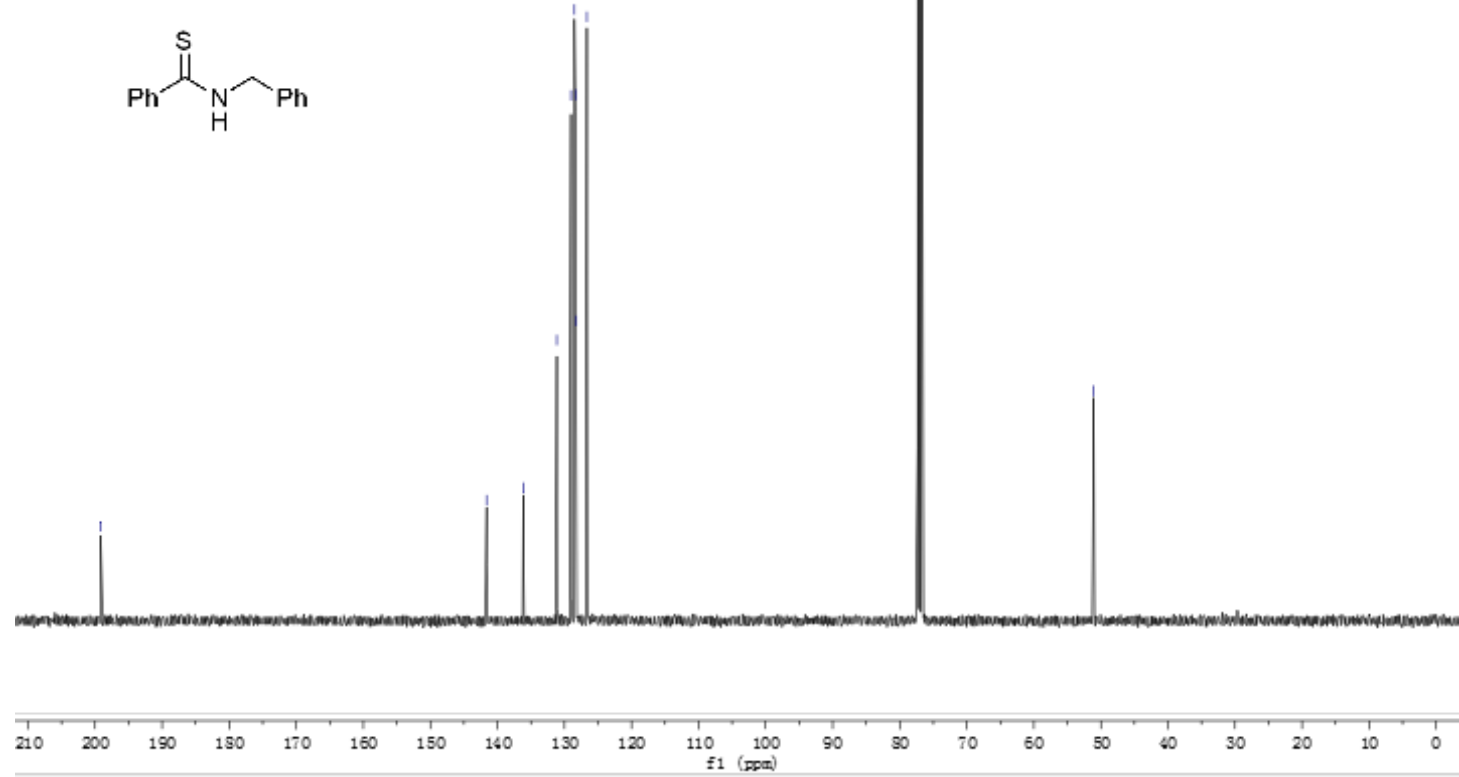


3d:

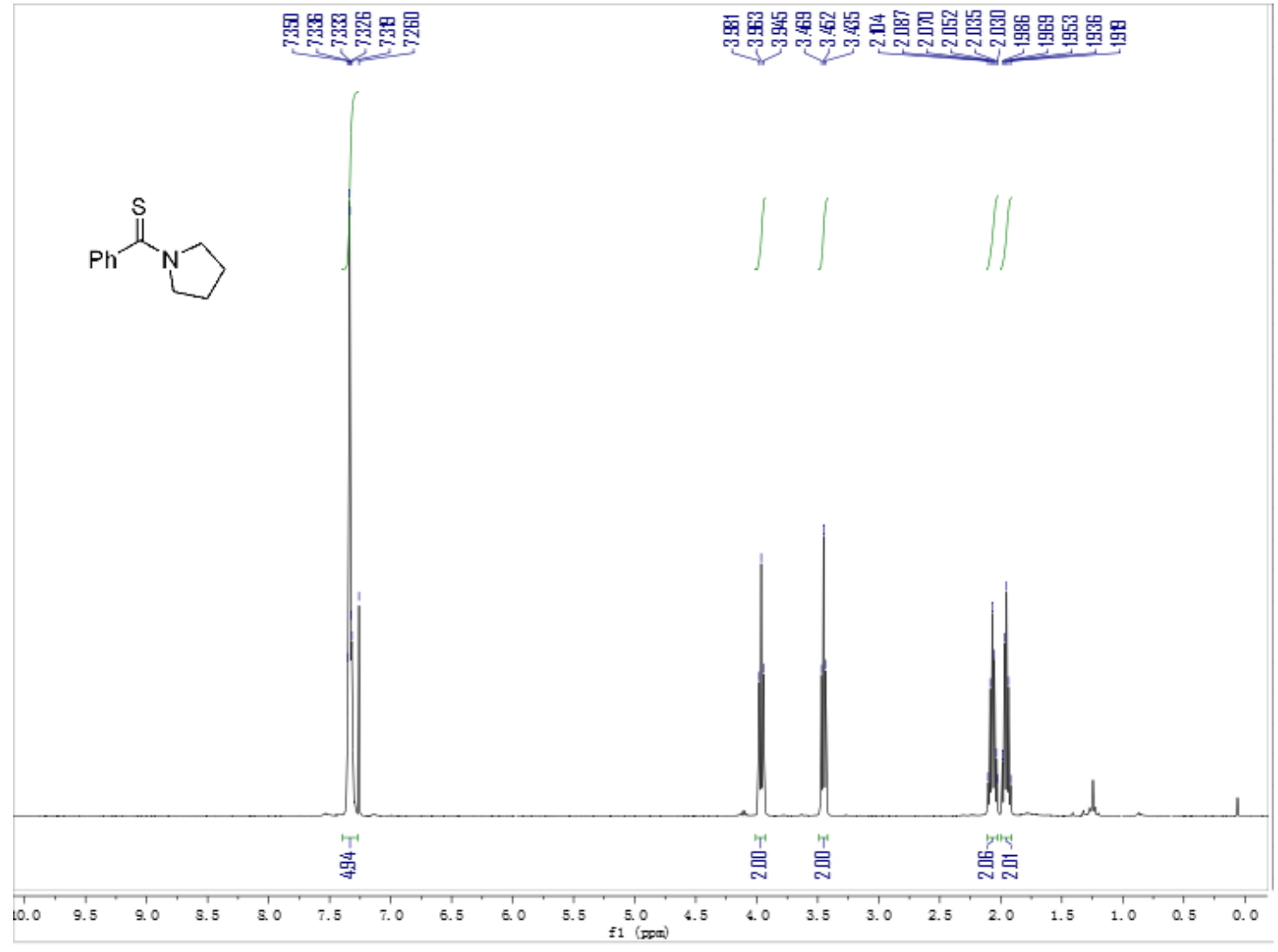

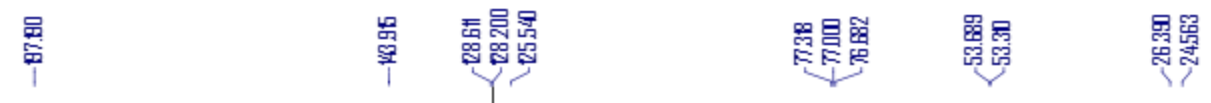

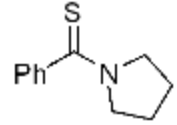

$210 \quad 200 \quad 190 \quad 130 \quad 170 \quad 160 \quad 150 \quad 140 \quad 130 \quad 120 \quad 110 \quad 100$ 
3e:

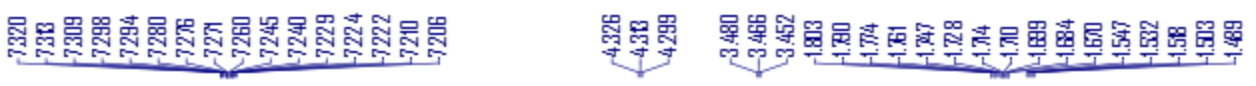
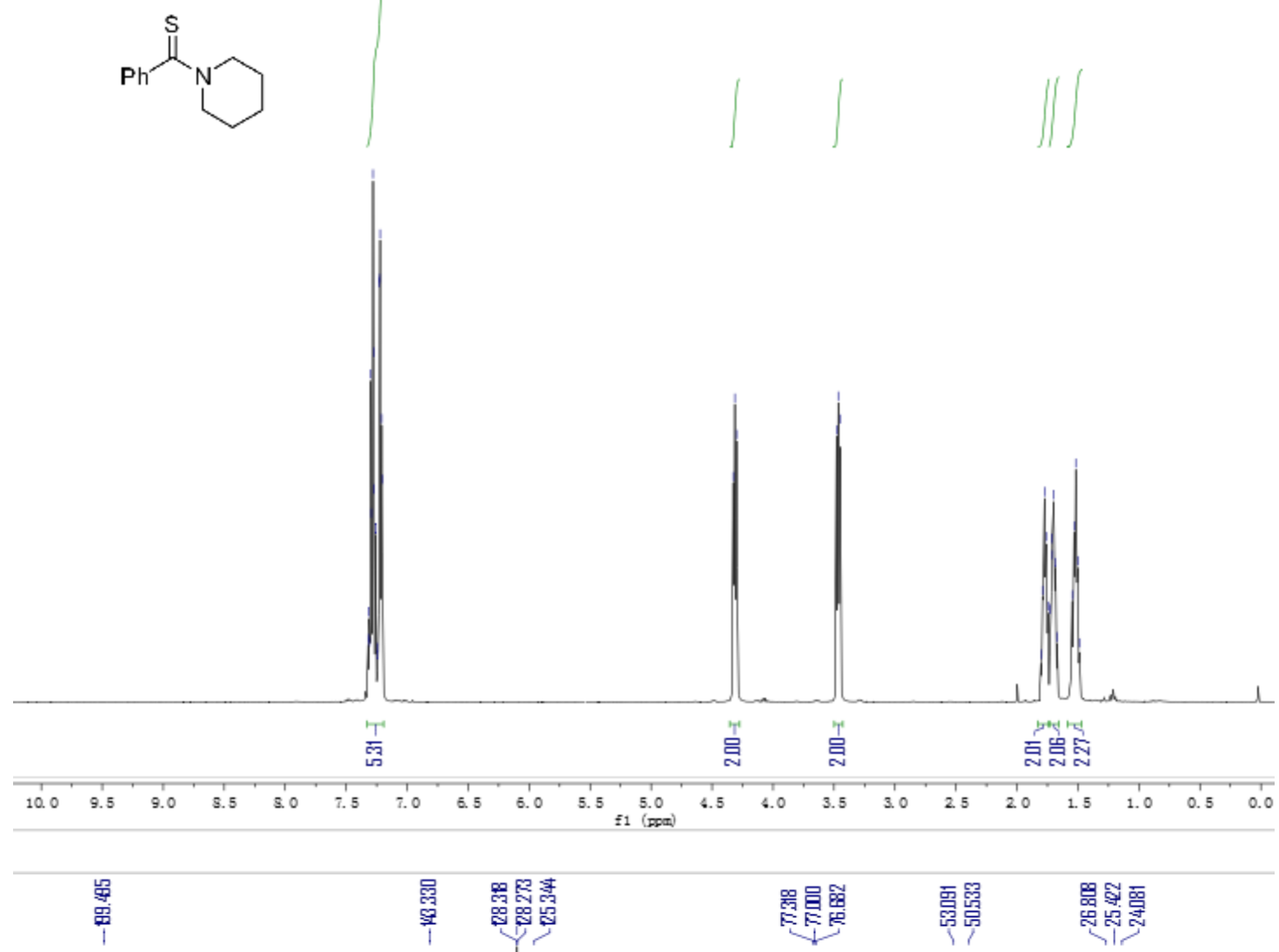

至

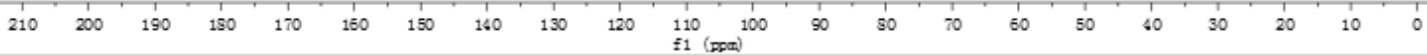


3f:
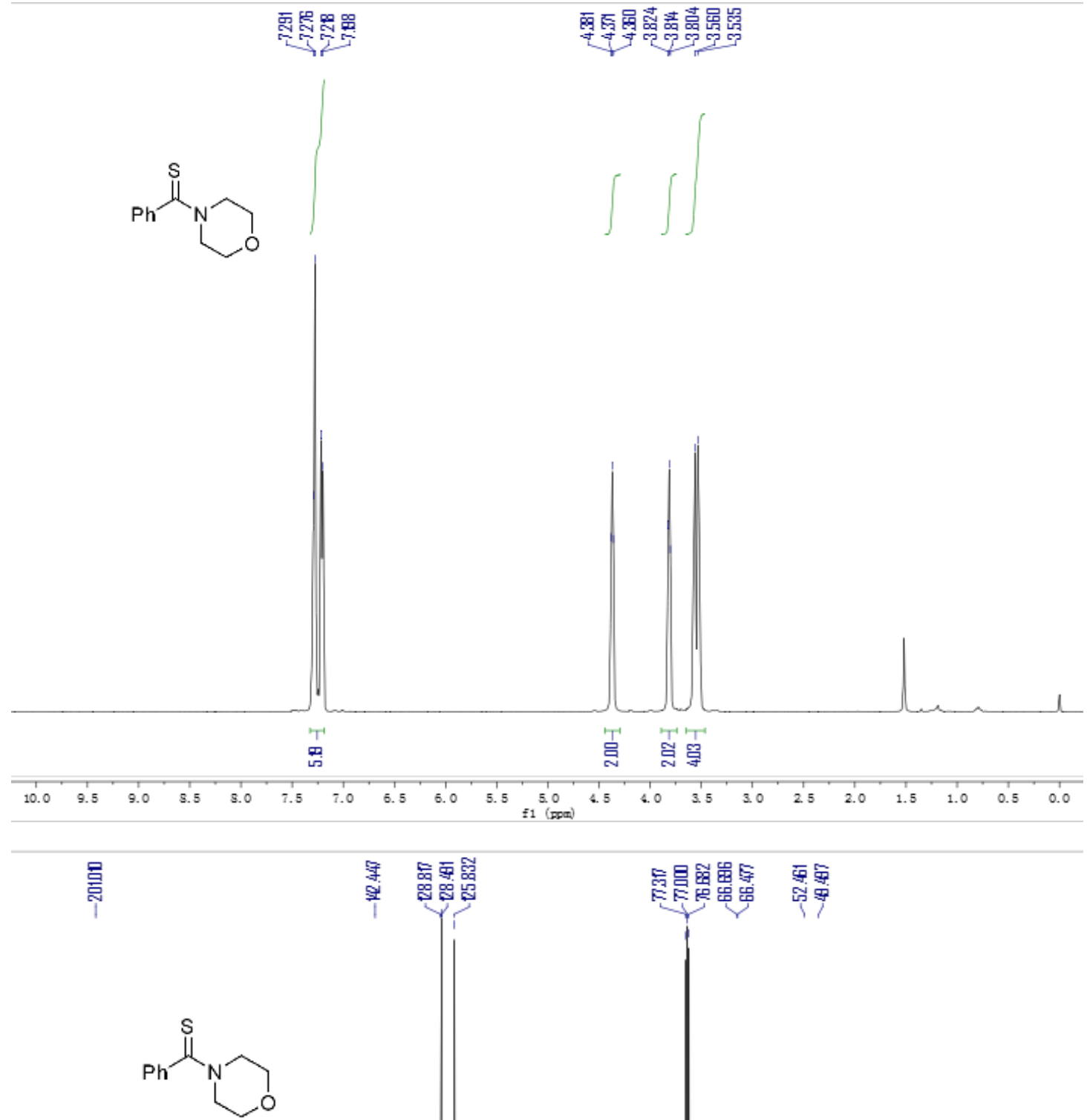

発舜嵒

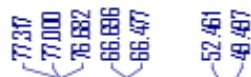

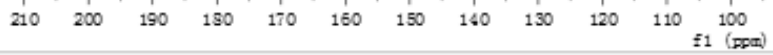

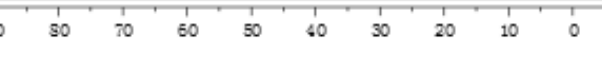


3g:
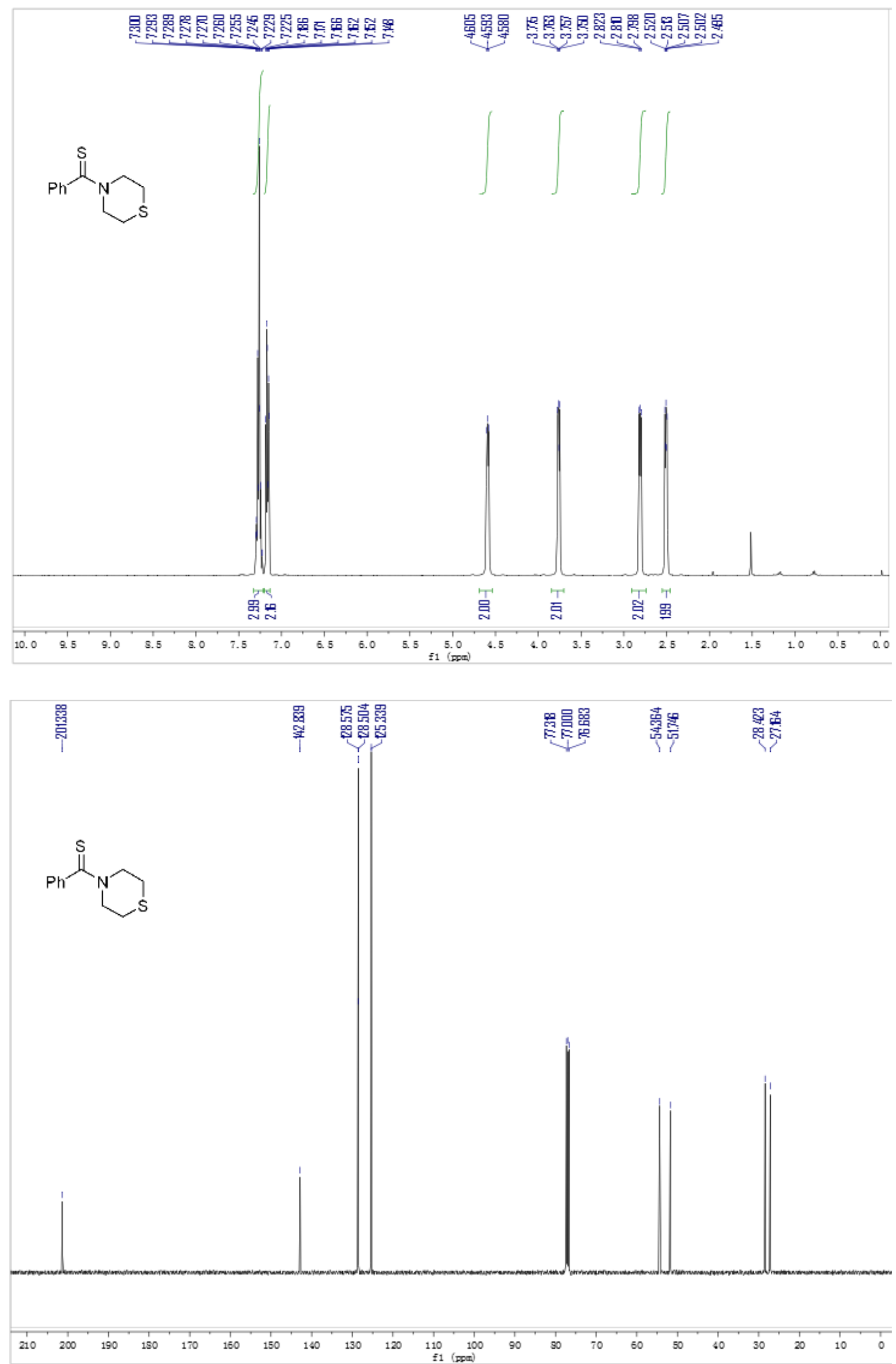
3h:

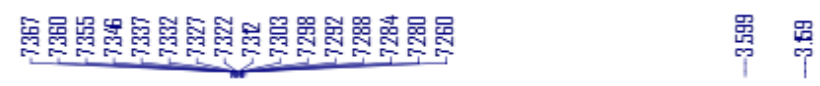<smiles>CN(C)C(=O)c1ccccc1</smiles>

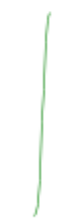

总言

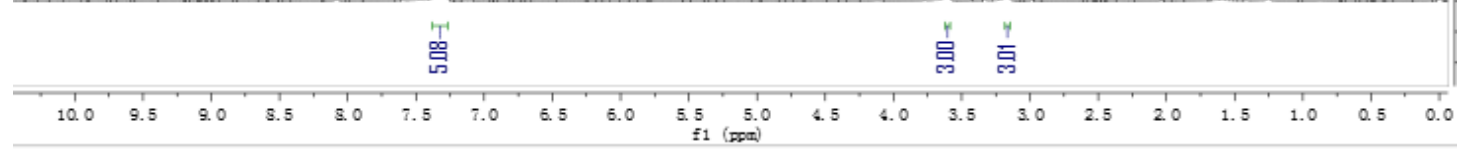

曽

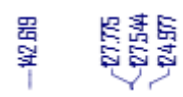

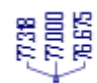

爵器

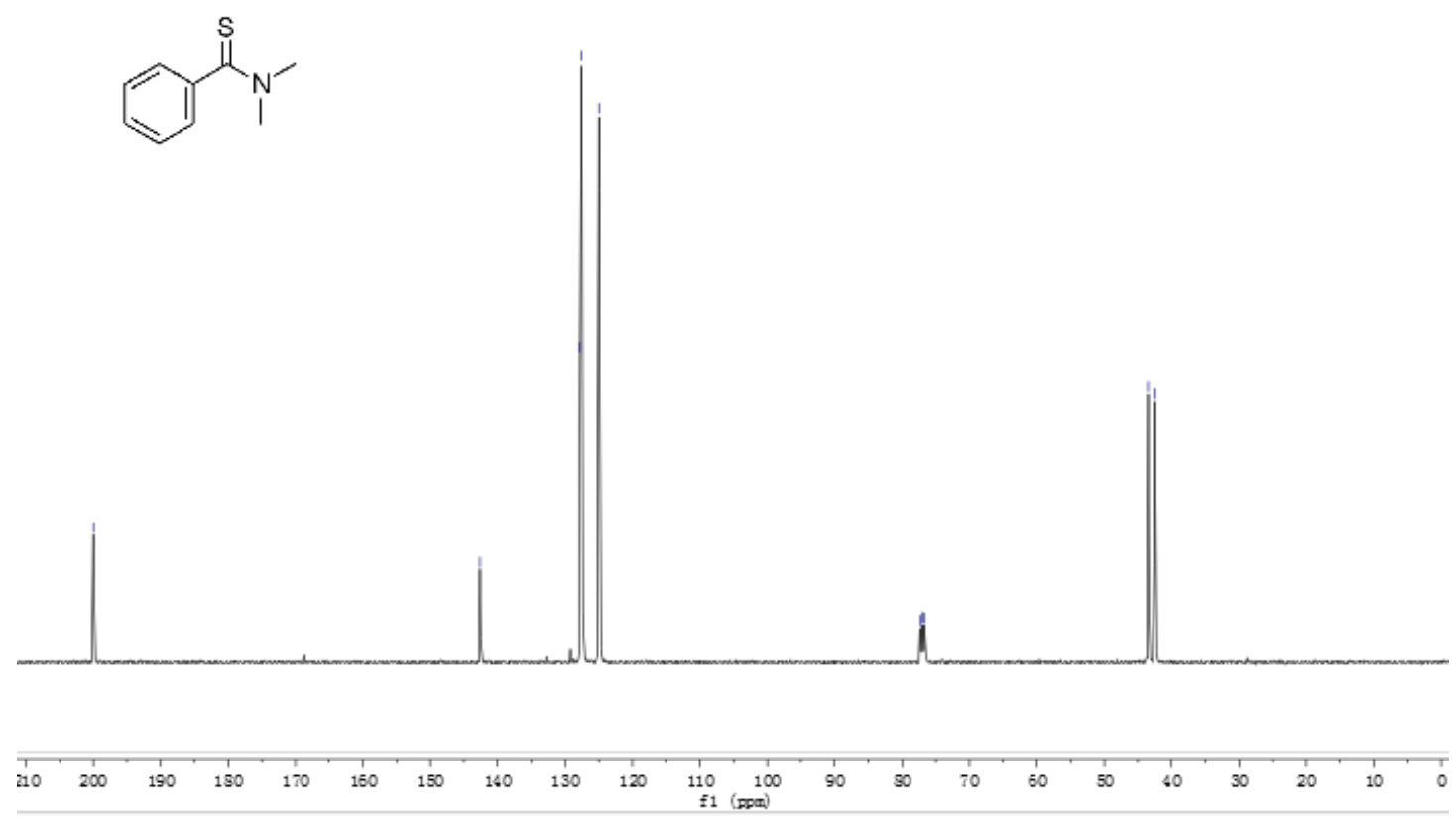


3i:

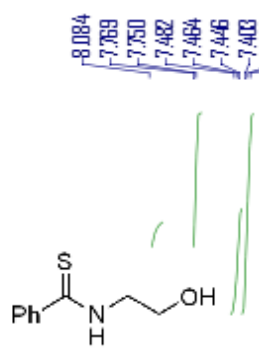

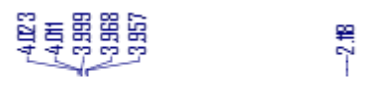

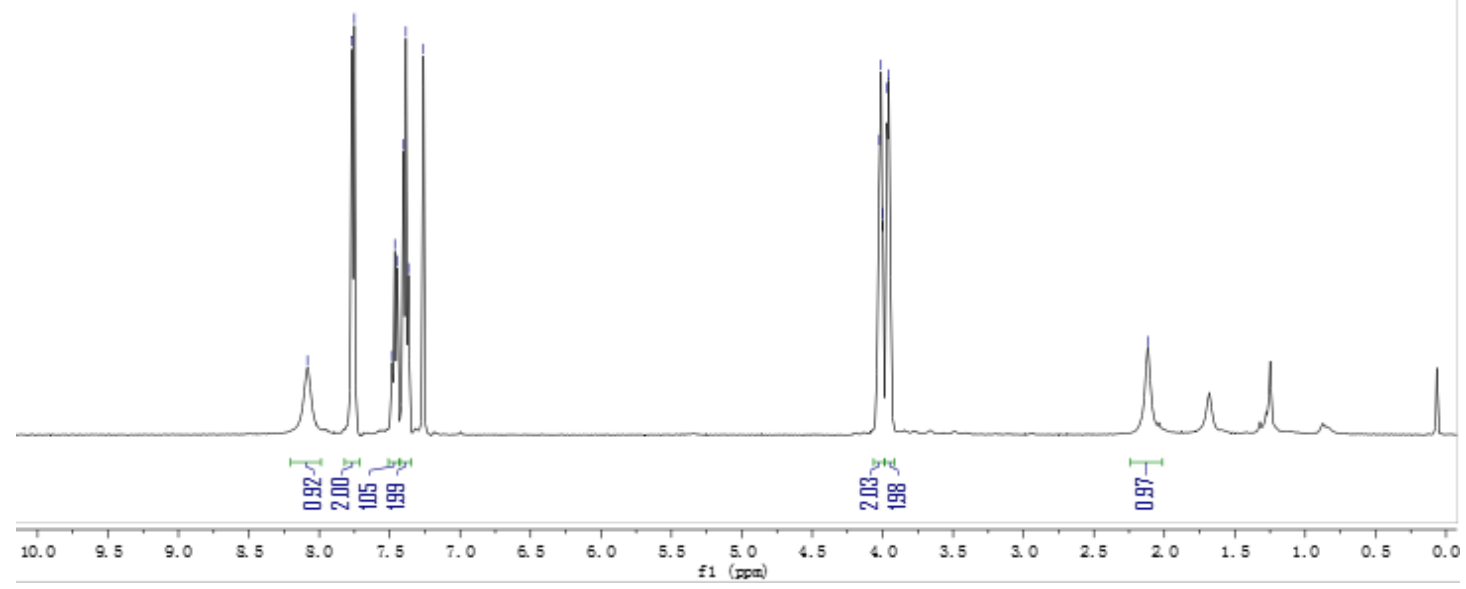

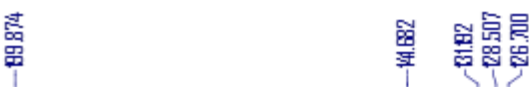

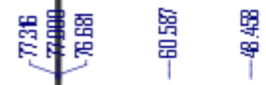

$\mathrm{Ph}_{\mathrm{H}}^{\mathrm{S}} \overbrace{\mathrm{OH}}^{\mathrm{O}}$

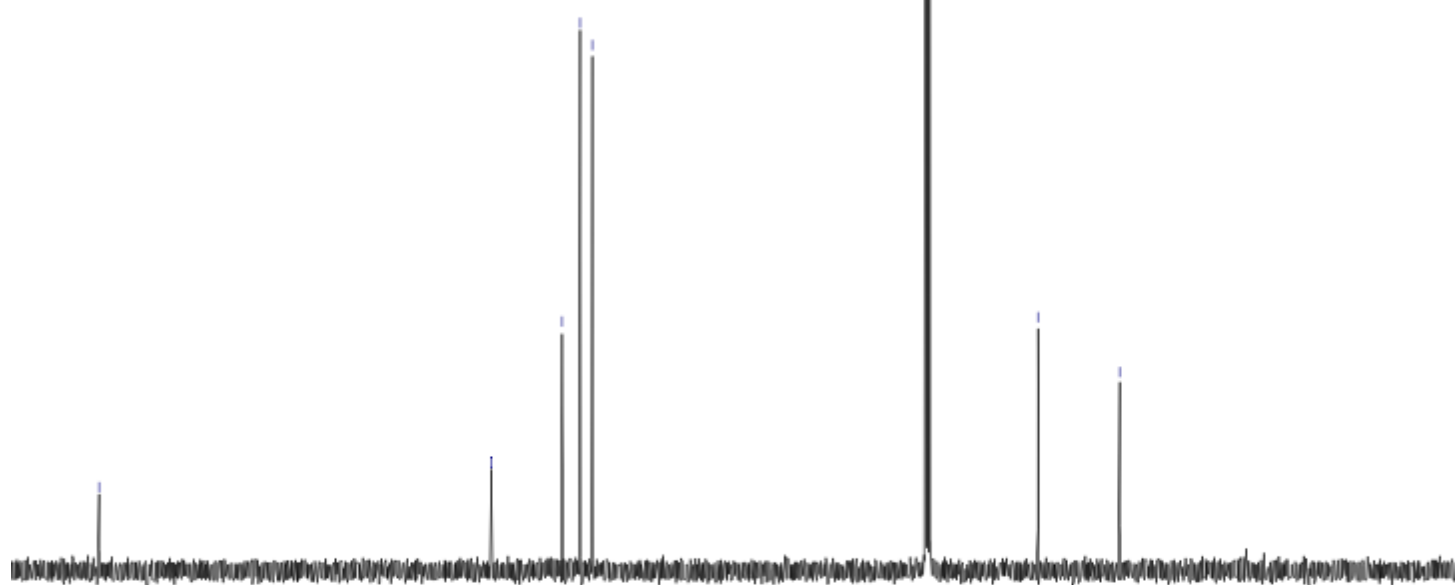

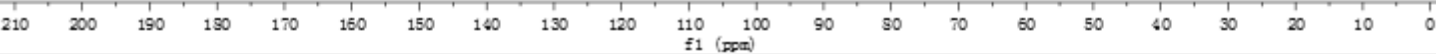


3j:

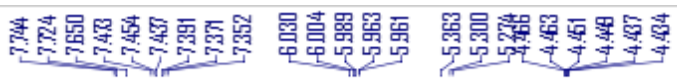
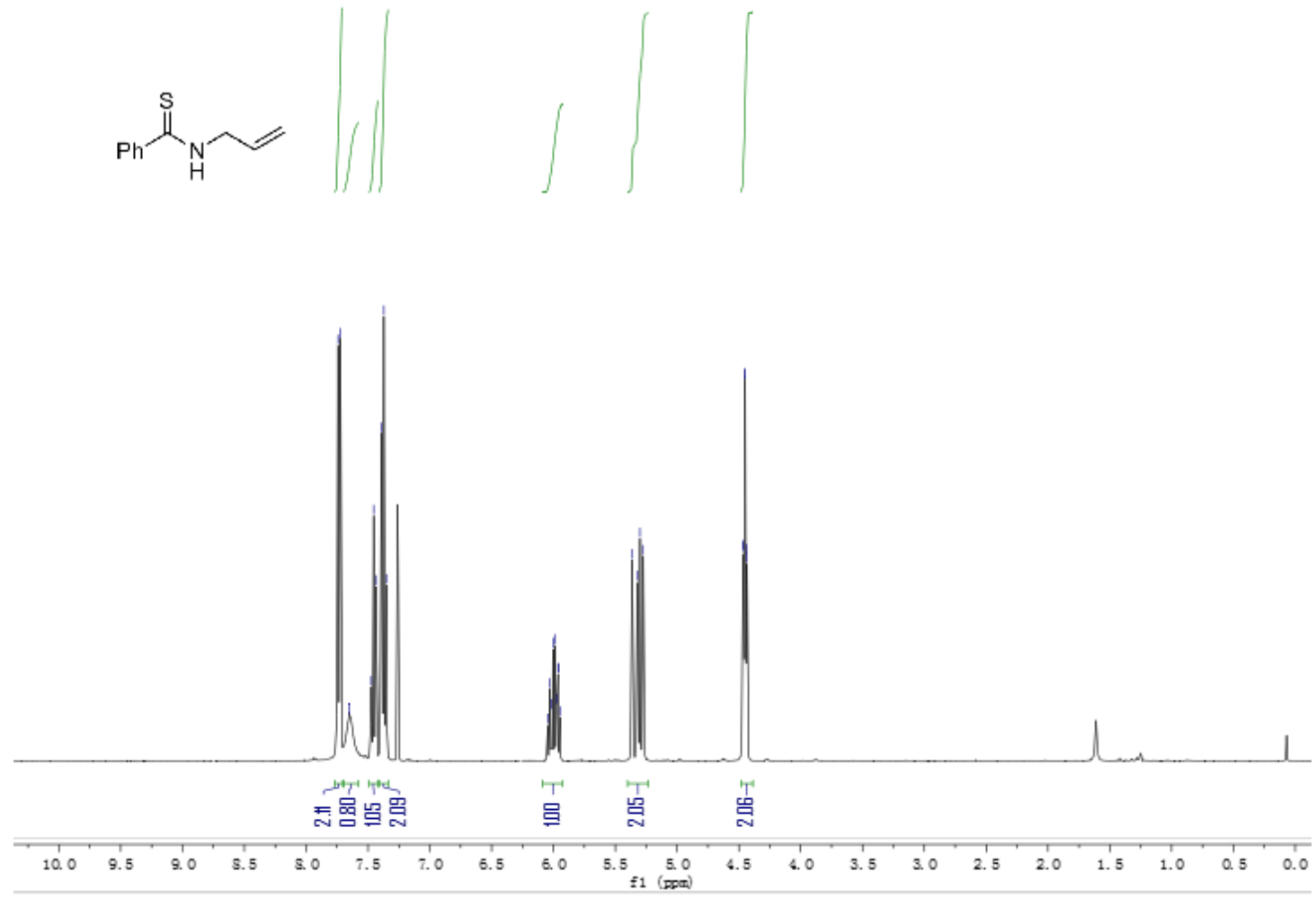

忿

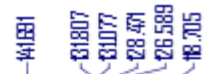

空员嵒

踹

$\mathrm{Ph}^{\mathrm{S}} \widehat{\mathrm{H}}$
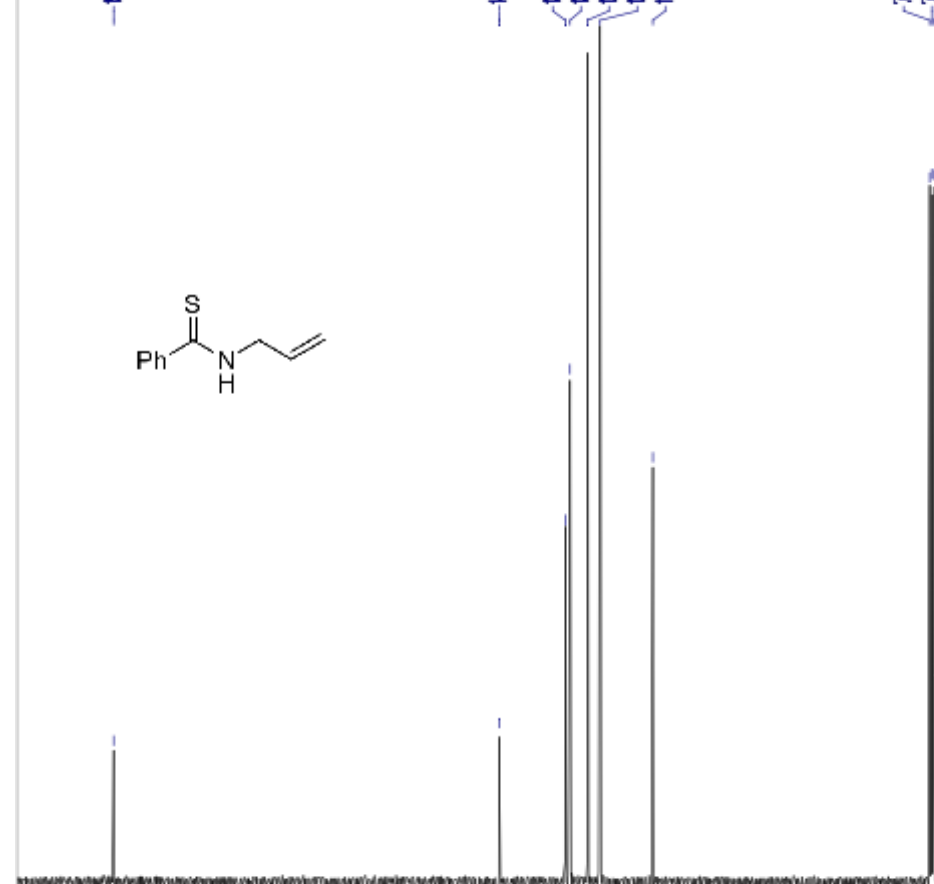

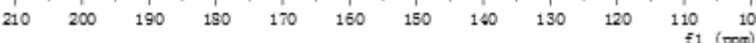


3k:

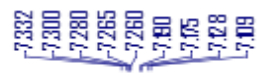
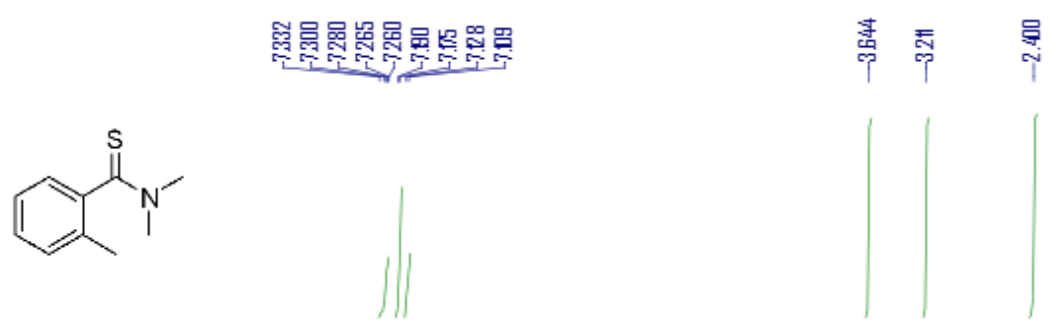

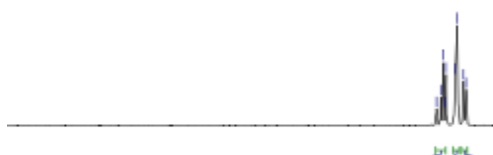

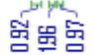

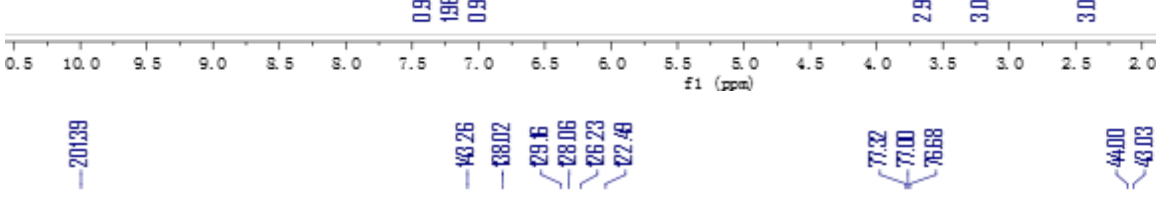

盖

管
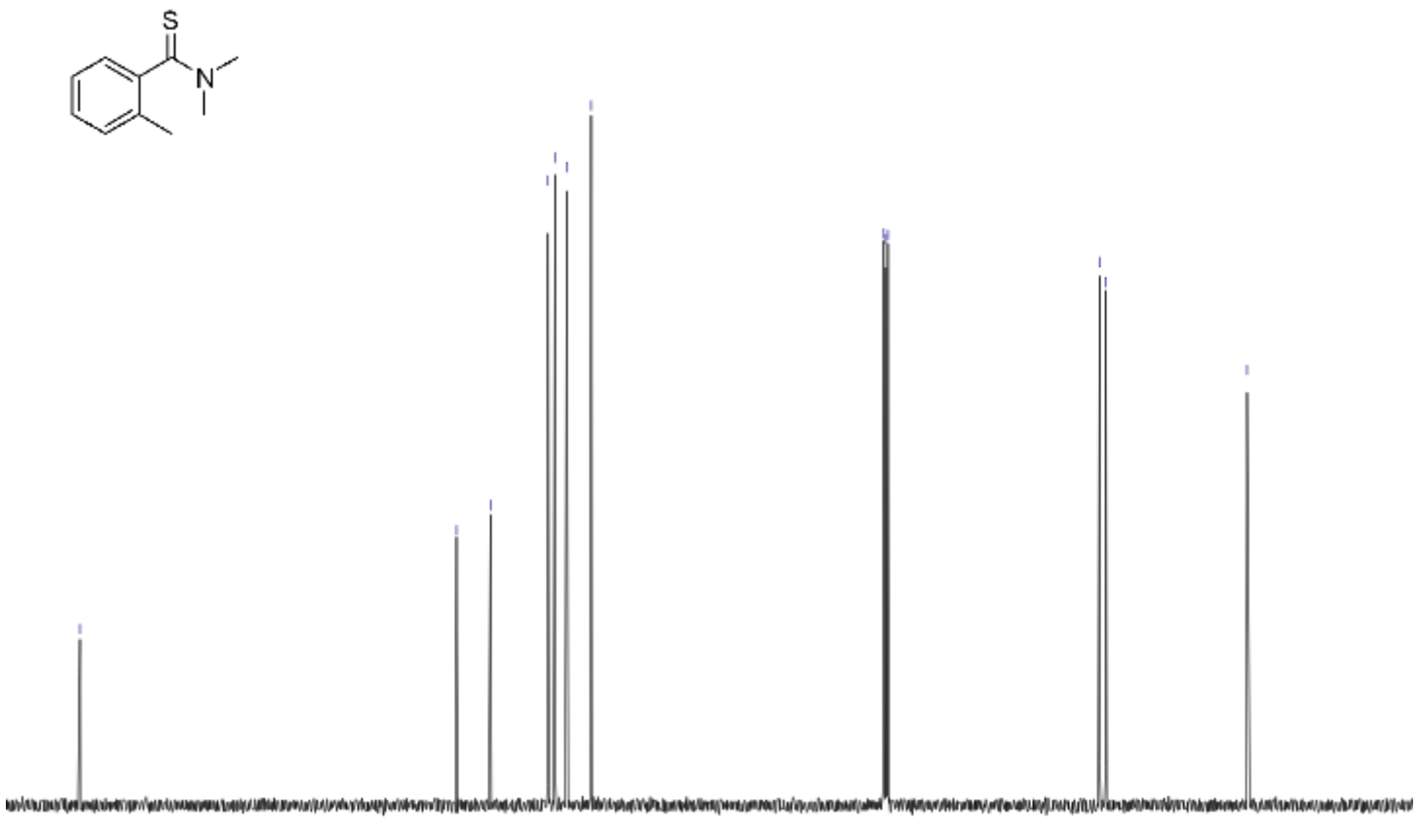

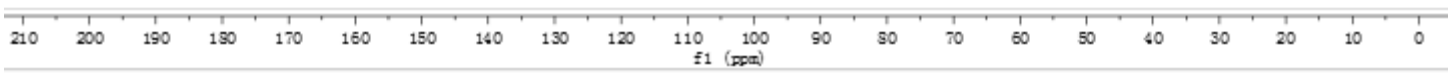

55 
31:

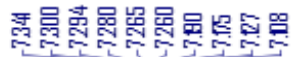
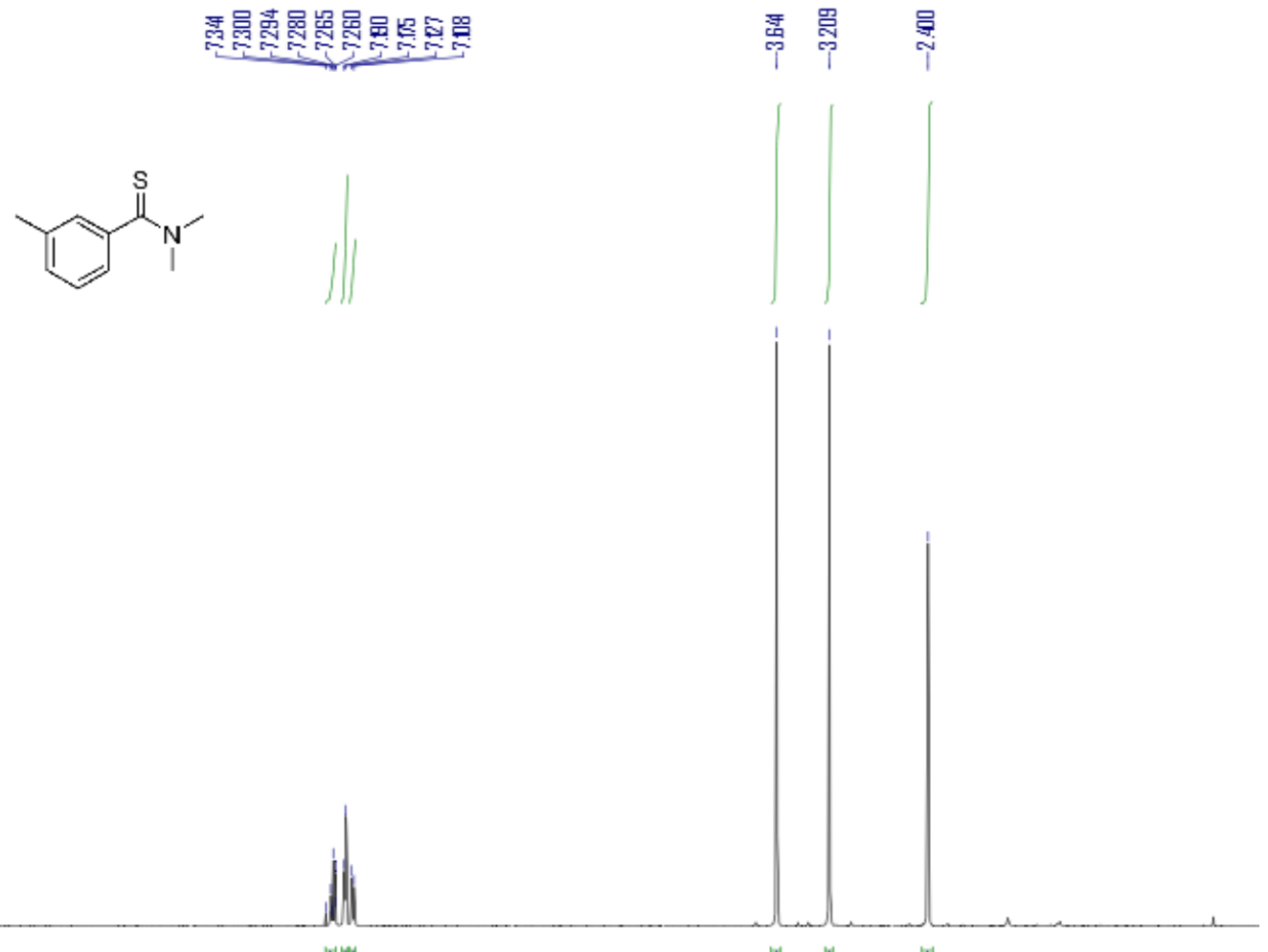

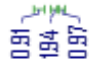

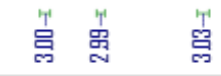

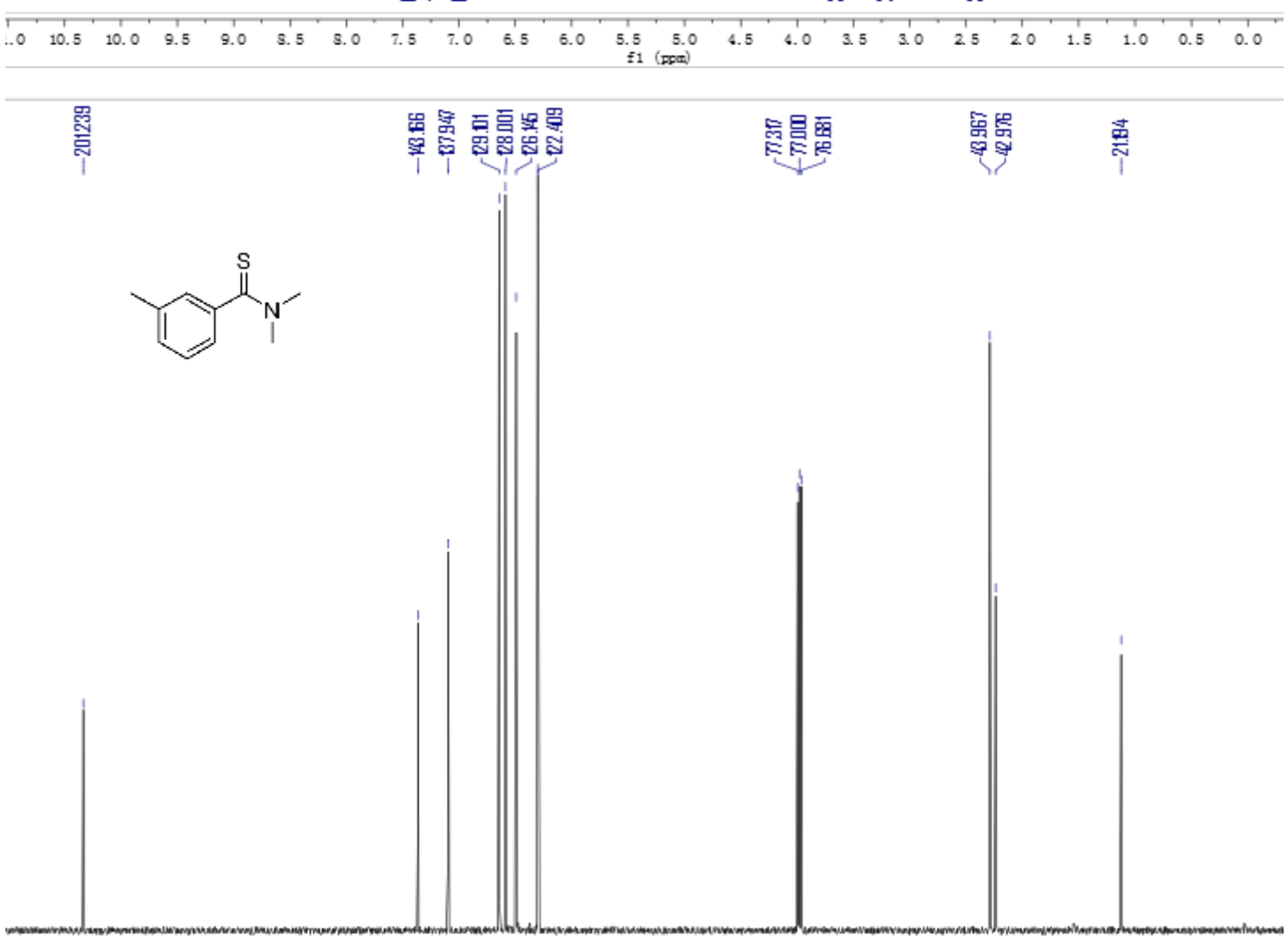

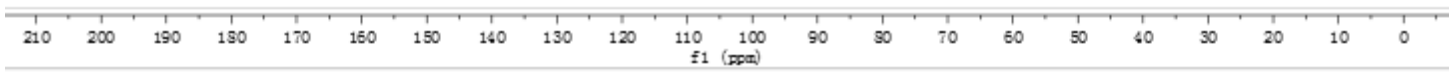

56 
3m:

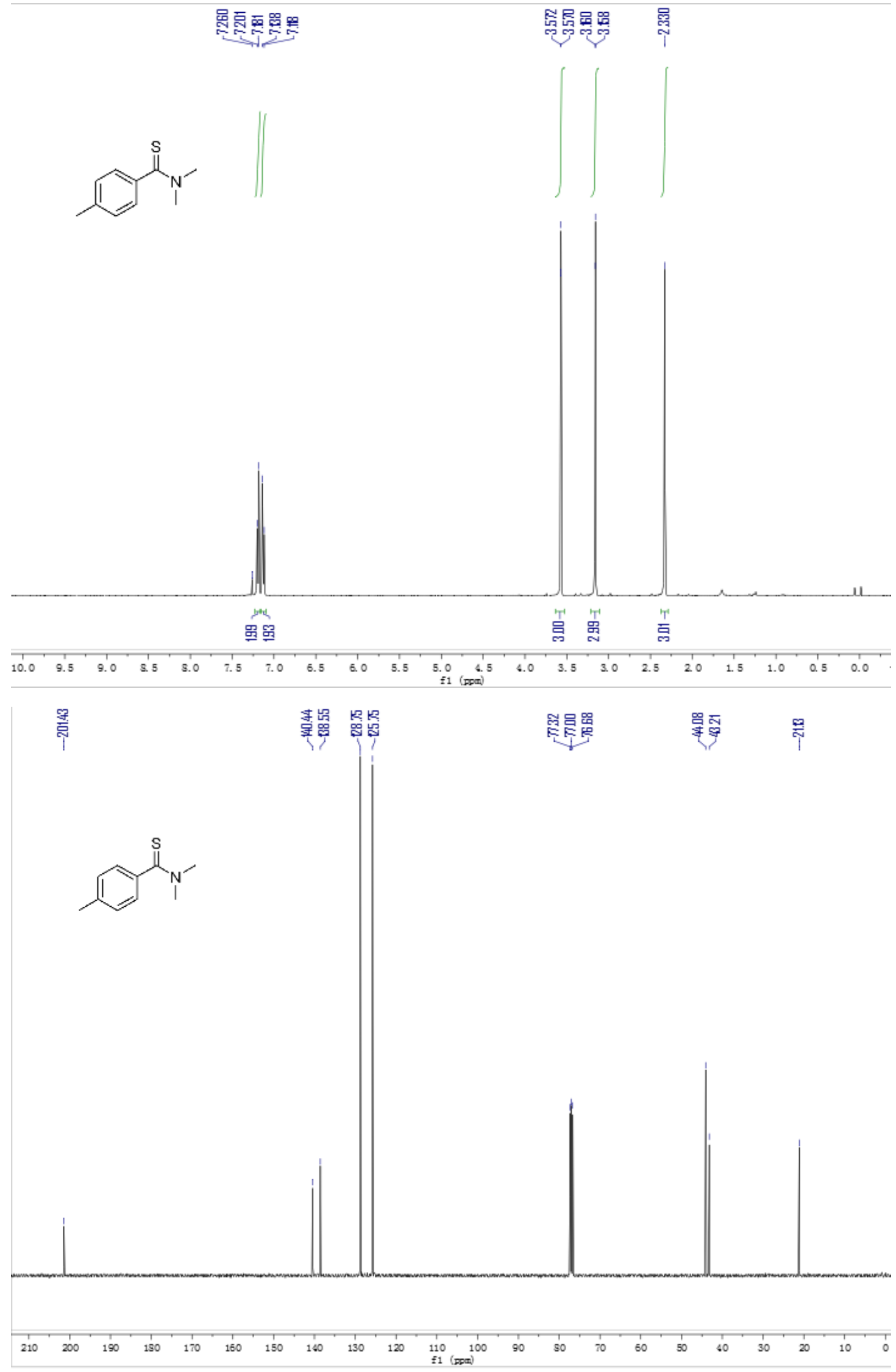


3n:

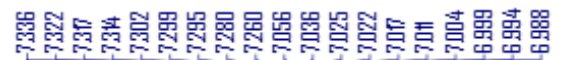
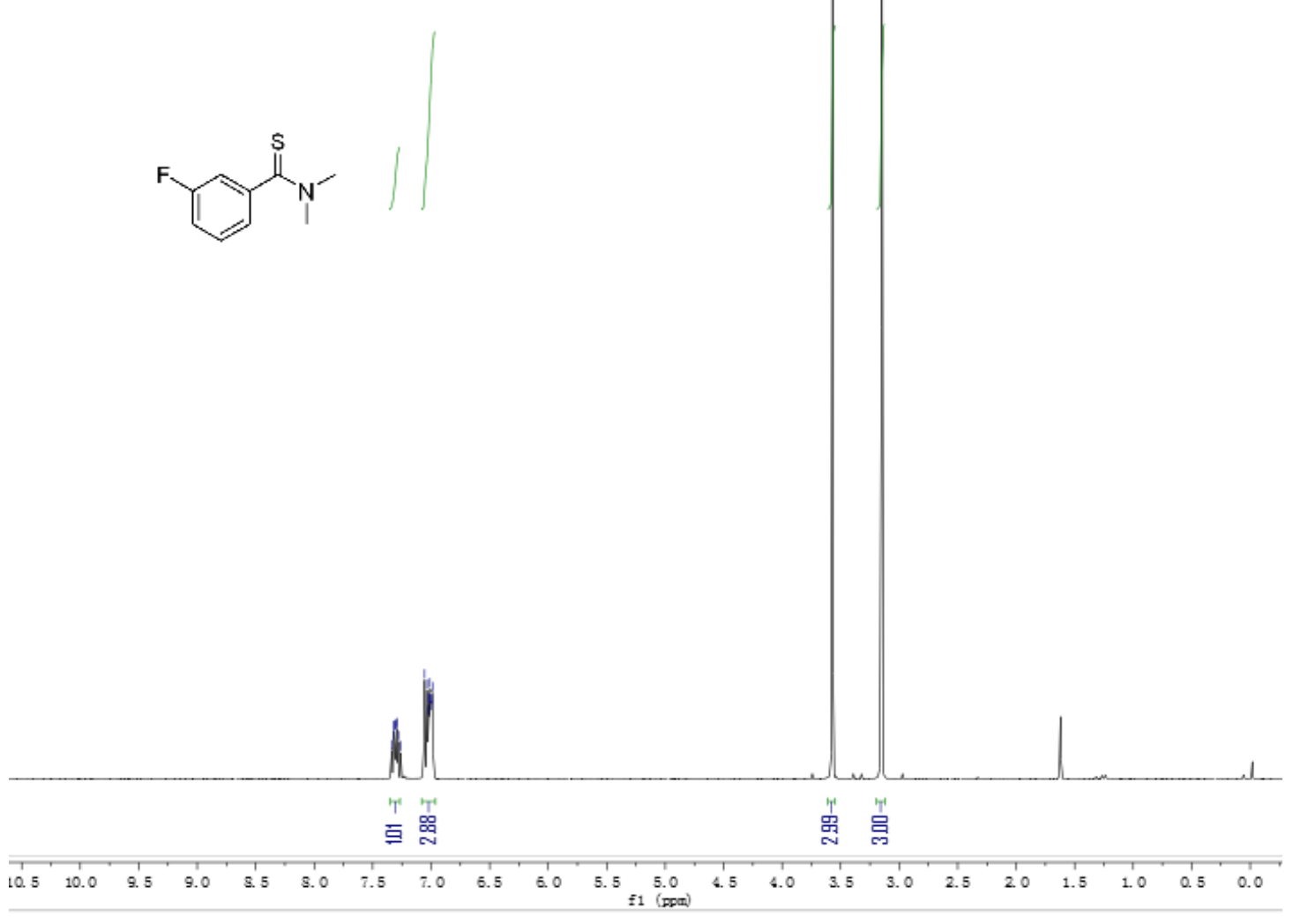

营

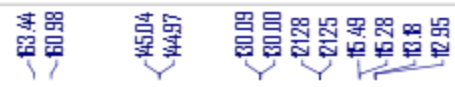

最罡
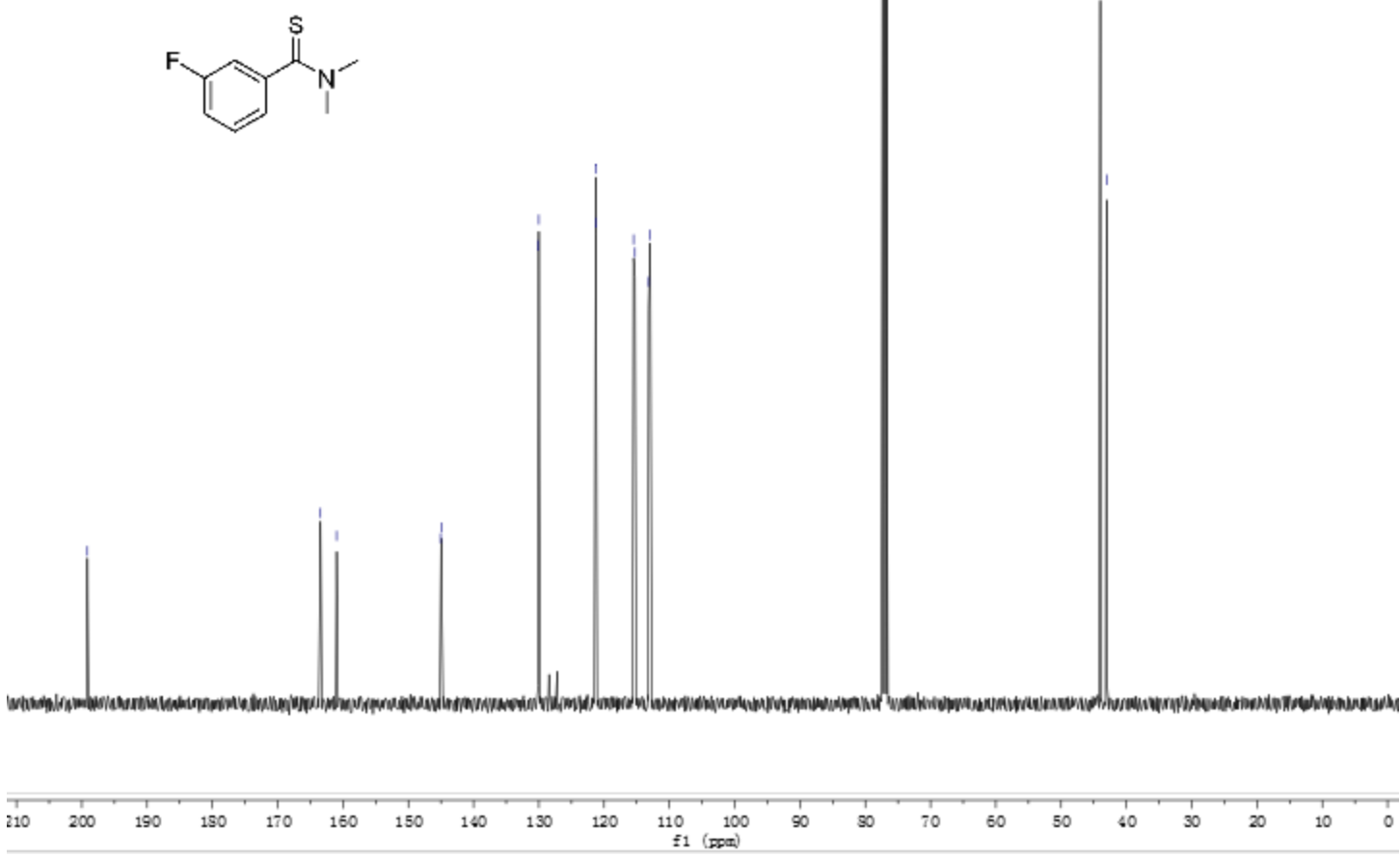

58 
뭄
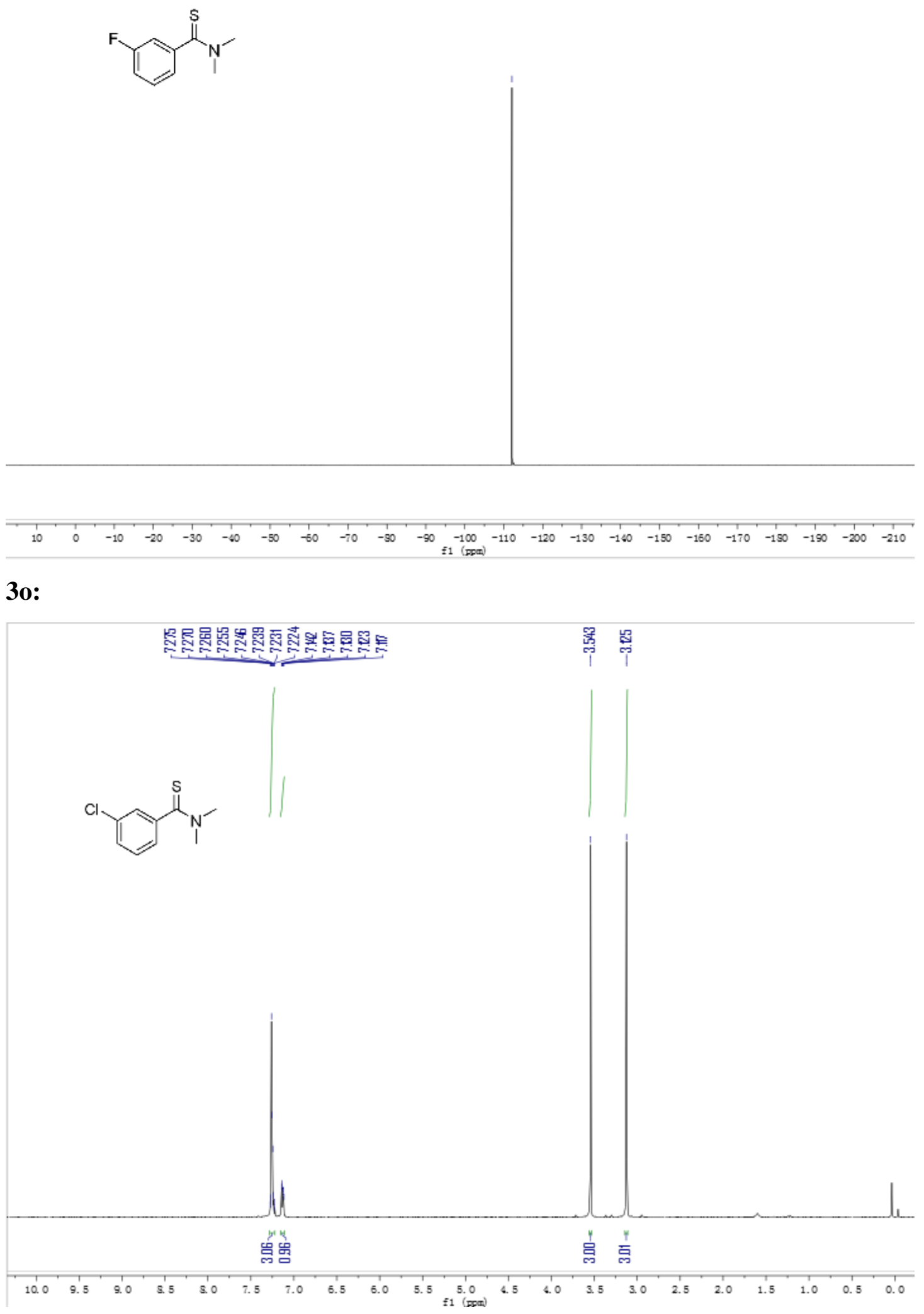

59 

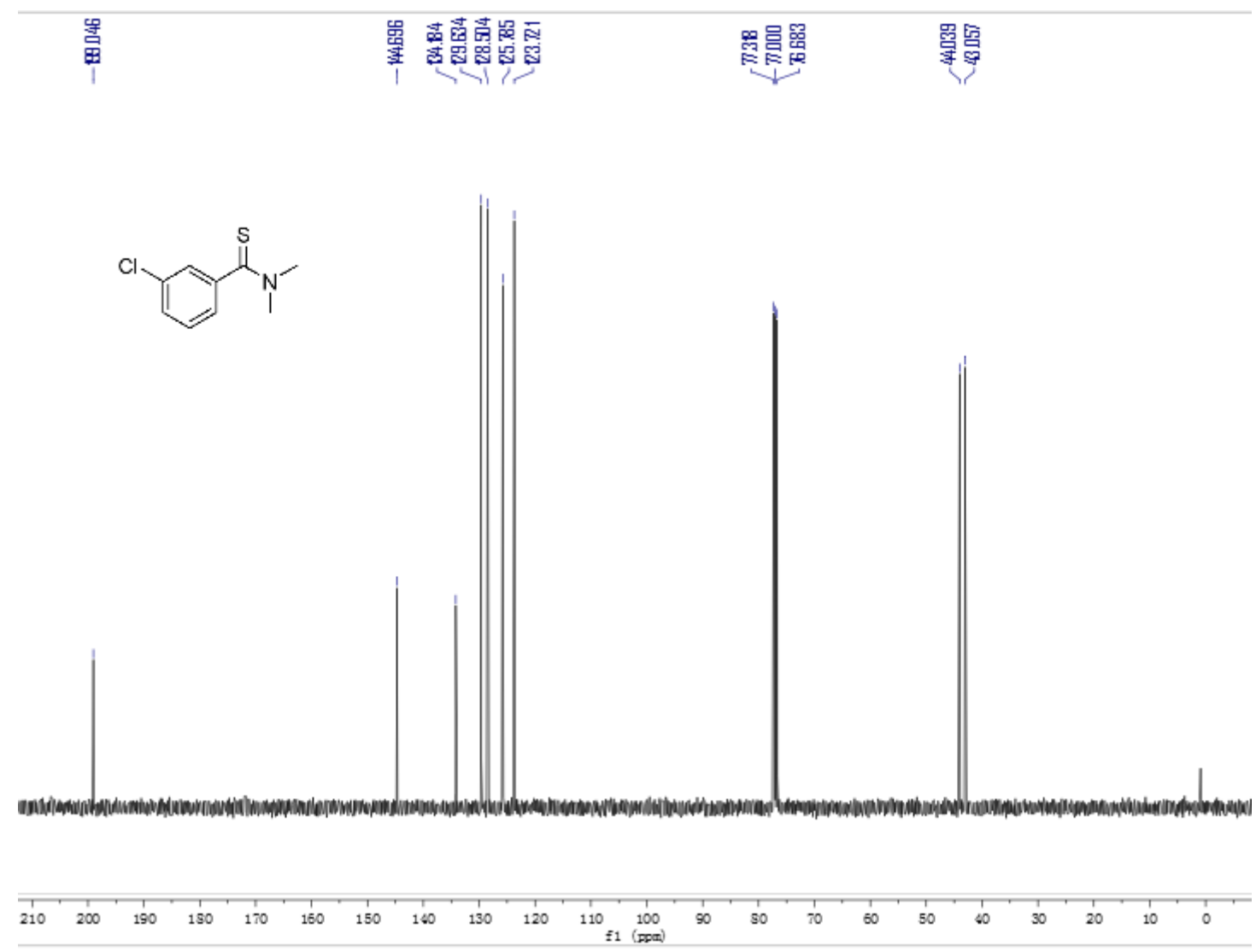

3p:

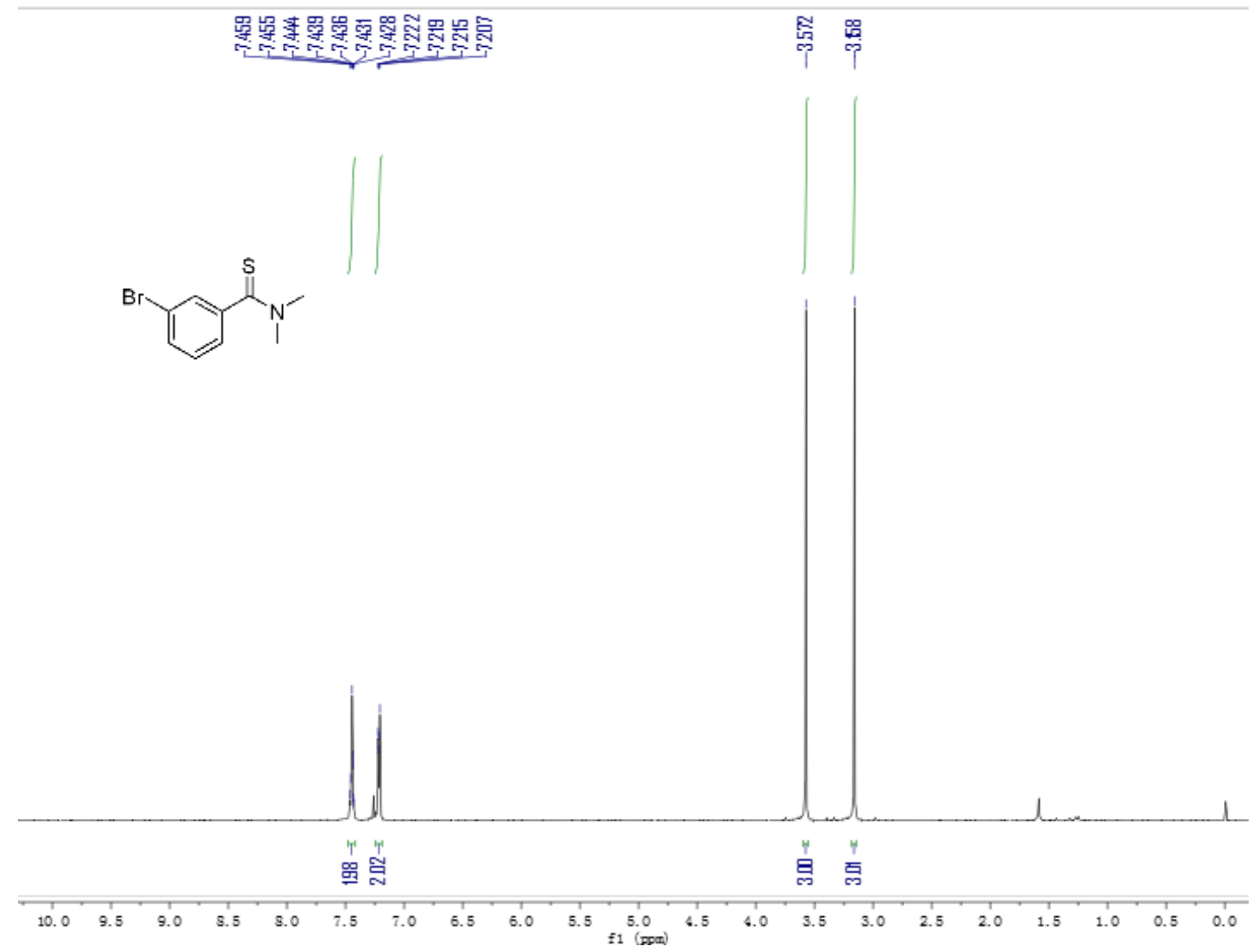



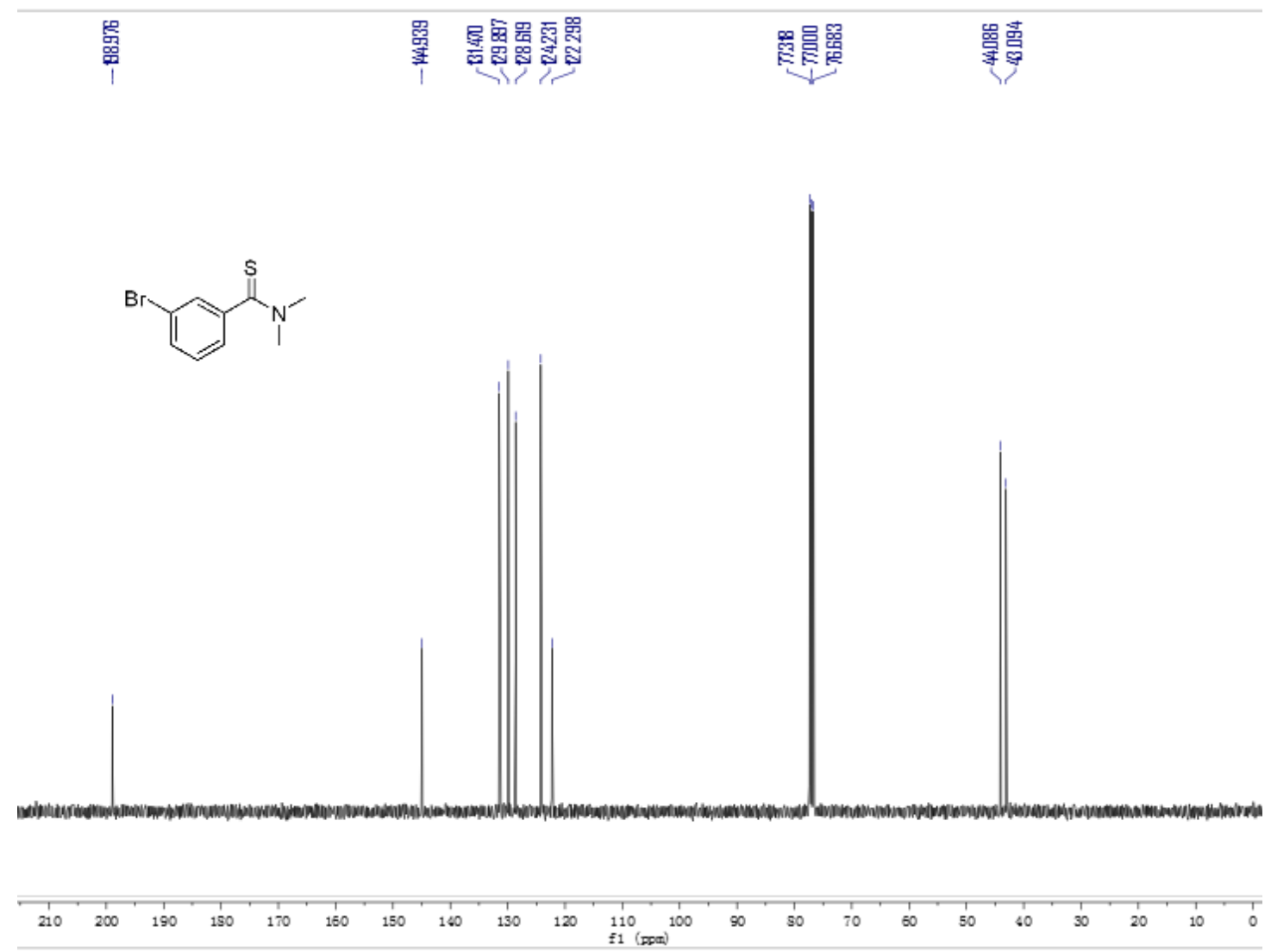

3q:<smiles>COc1ccc(C(=S)N(C)C)cc1</smiles>

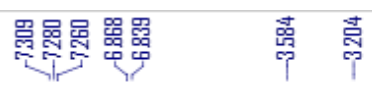

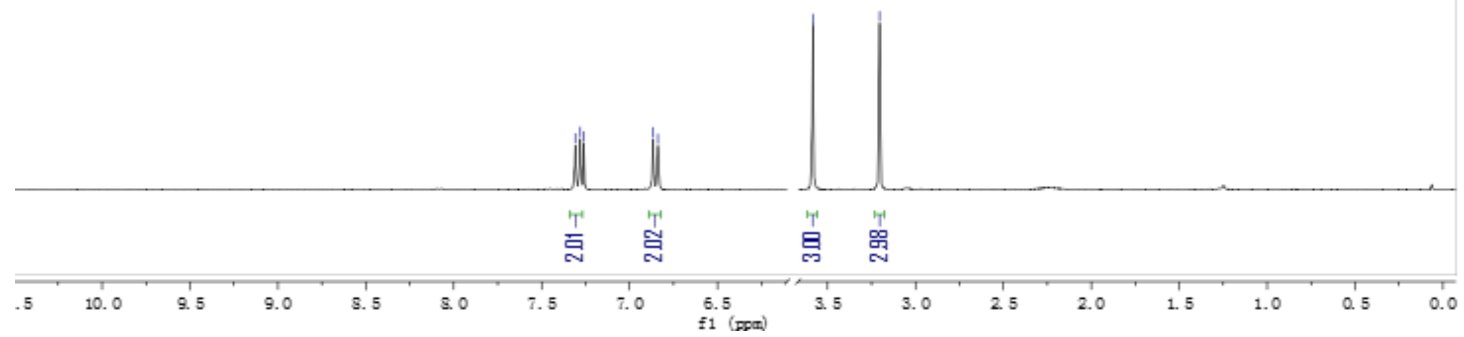




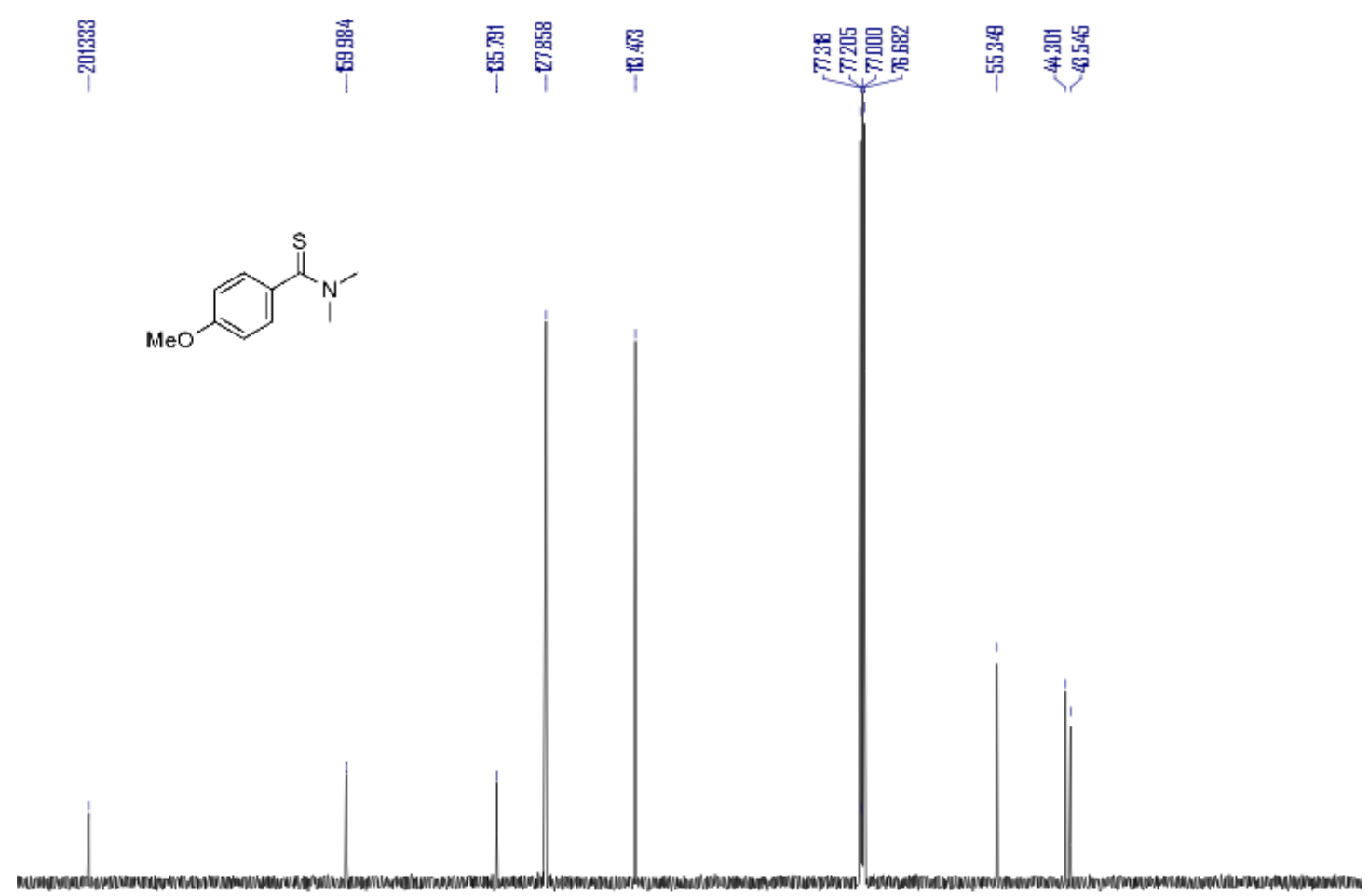

\begin{tabular}{llllllllllllllllllllll}
\hline 210 & 200 & 190 & 130 & 170 & 160 & 150 & 140 & 130 & 120 & 110 & 100 & 90 & 50 & 70 & 60 & 50 & 40 & 30 & 20 & 10 & 1 \\
\hline
\end{tabular}

3r:

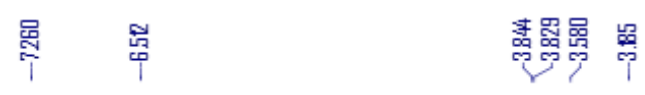<smiles>COc1cc(C(=S)N(C)C)cc(OC)c1OC</smiles>

穴

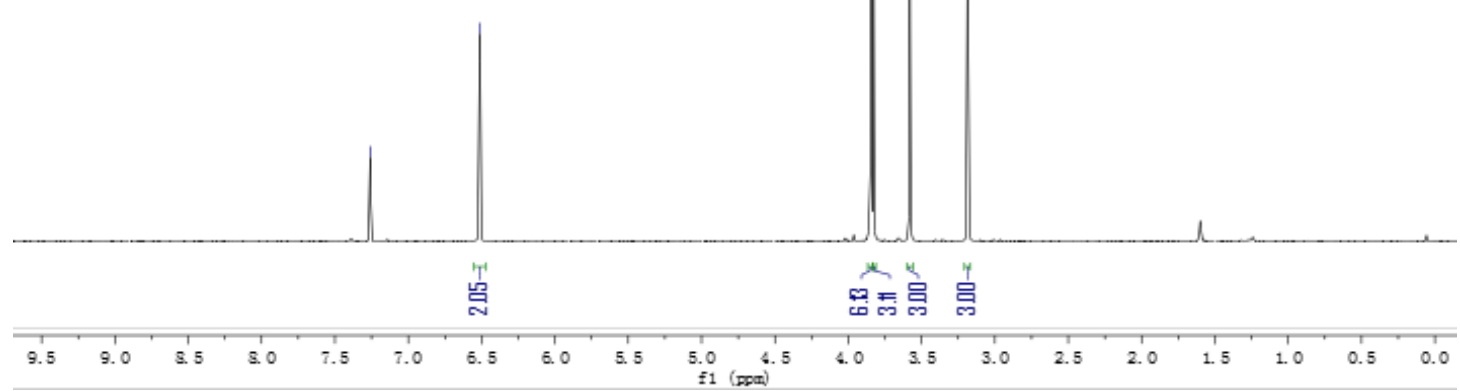




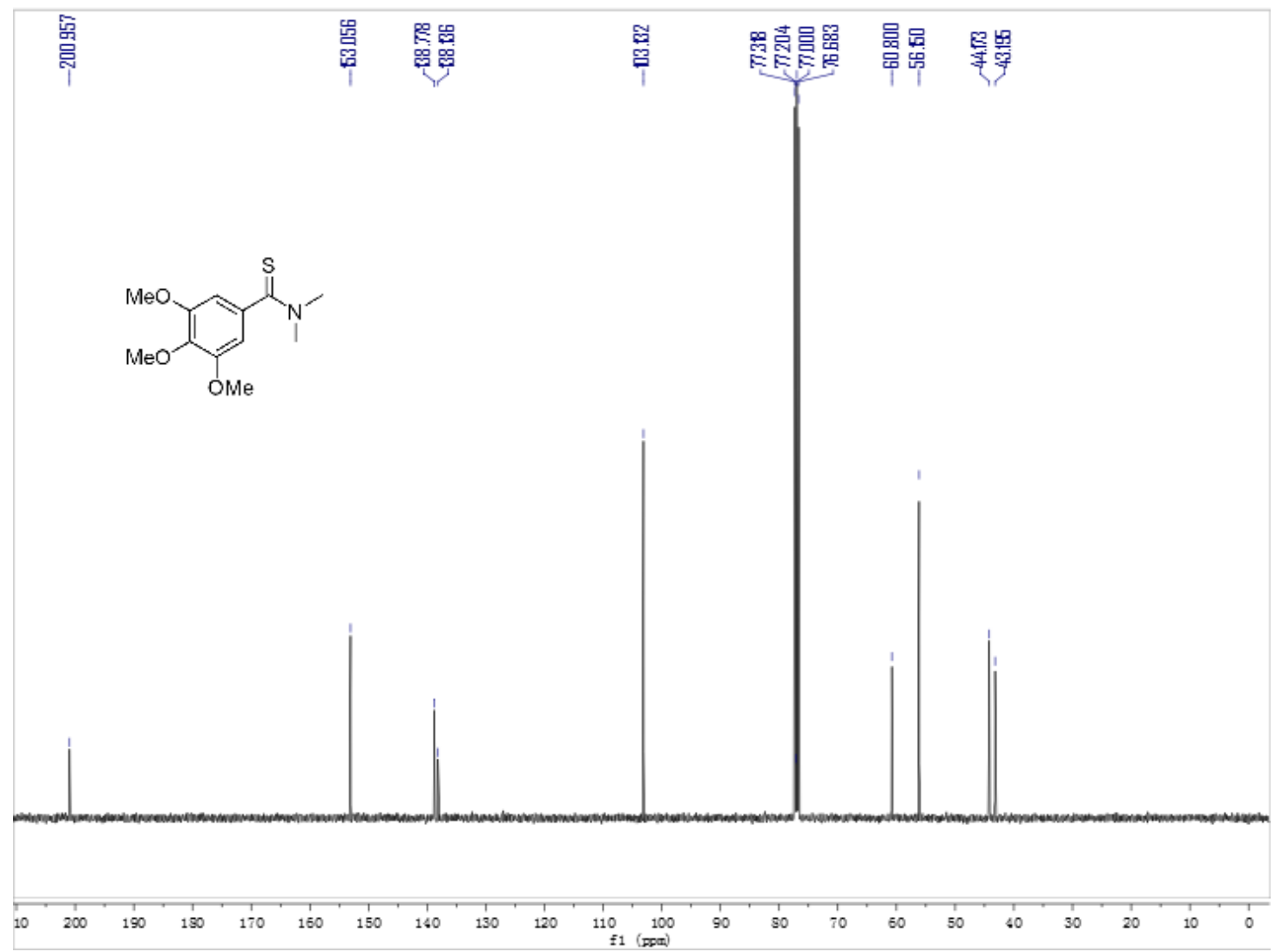

3s:<smiles>CN(C)C(=S)c1cccc(C(F)(F)F)c1</smiles>

嵓 㖕

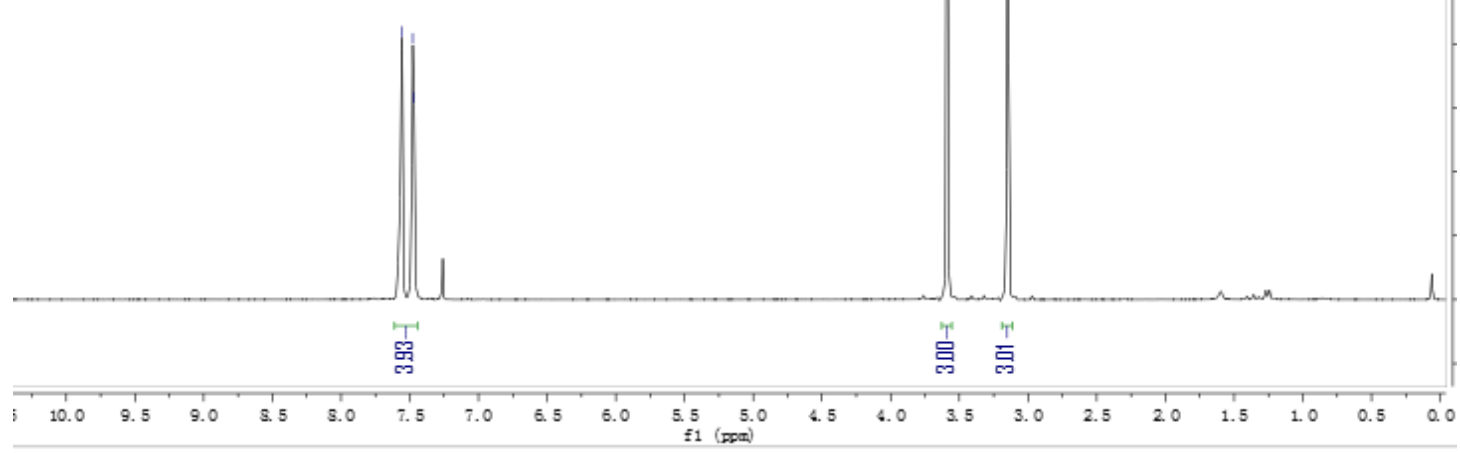



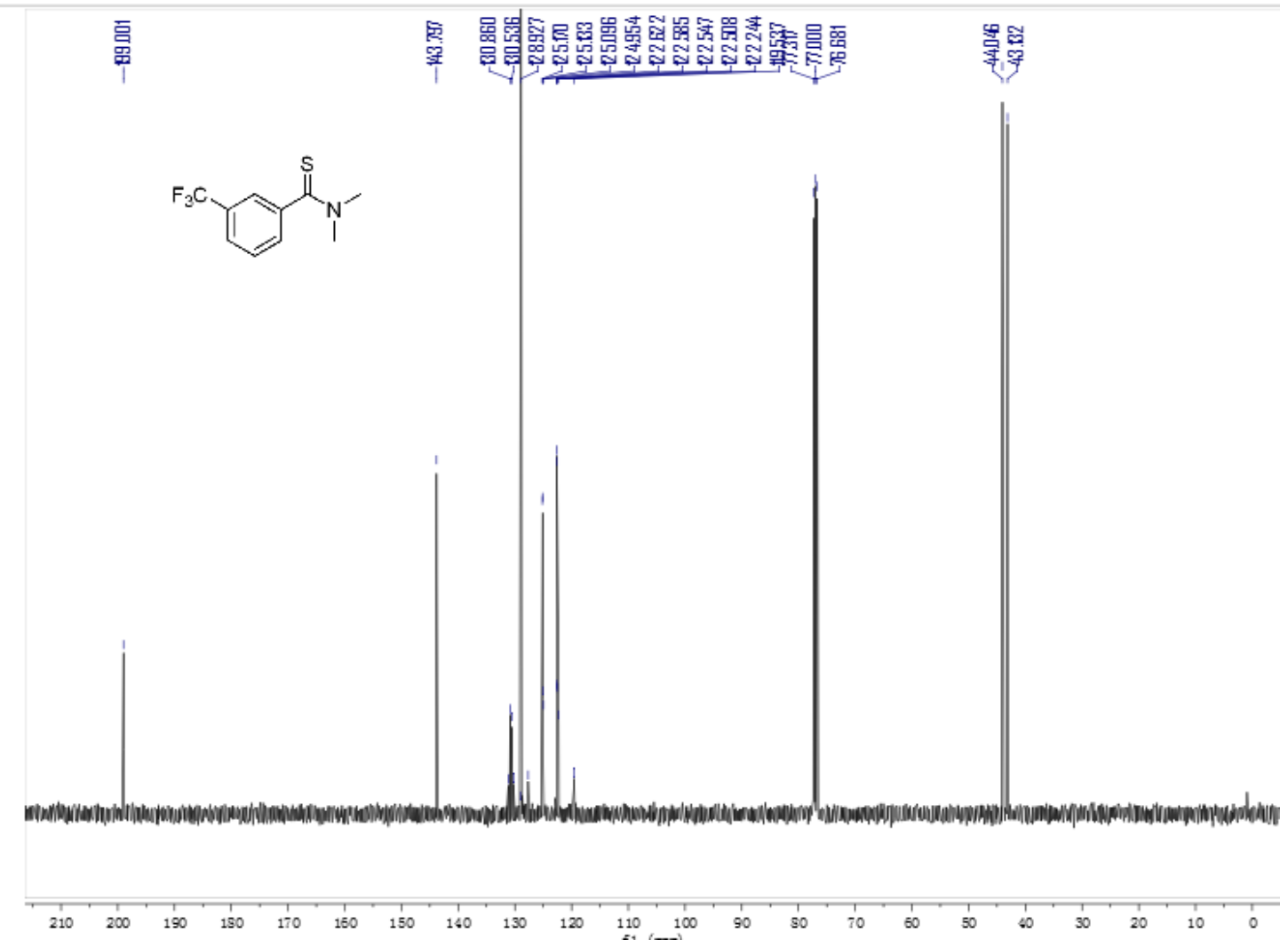

薄

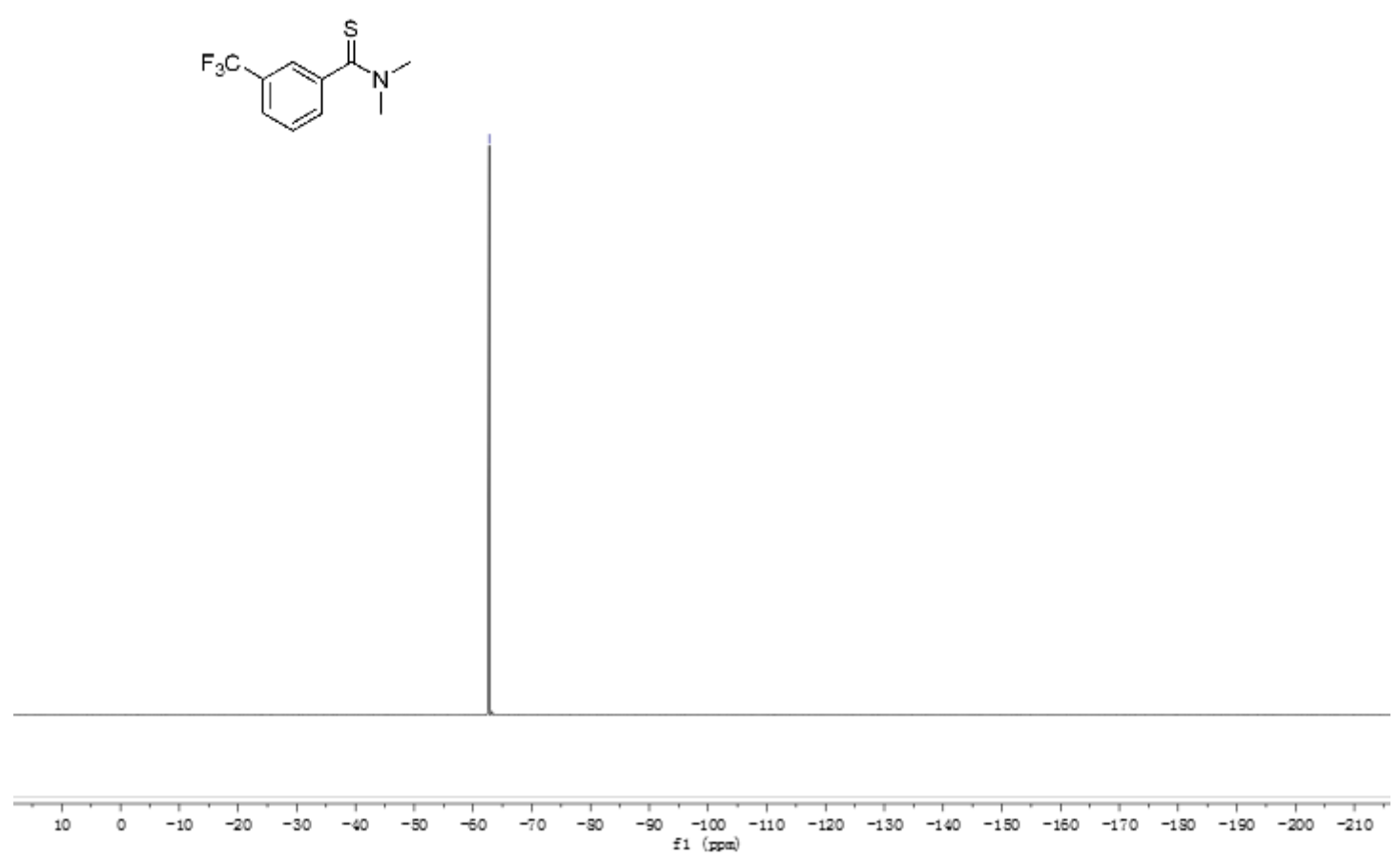


3t:

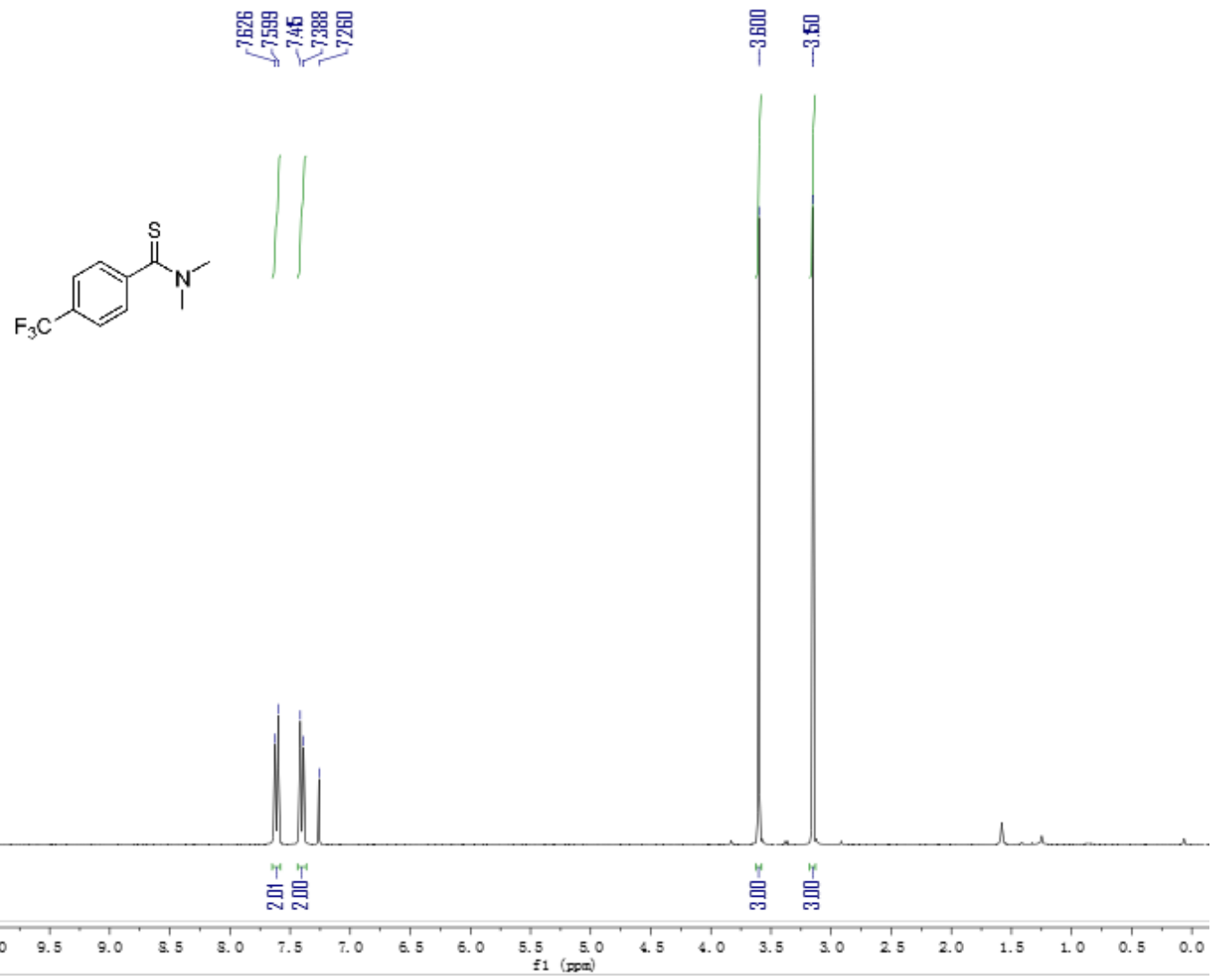

囬

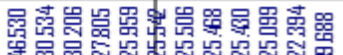

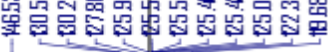
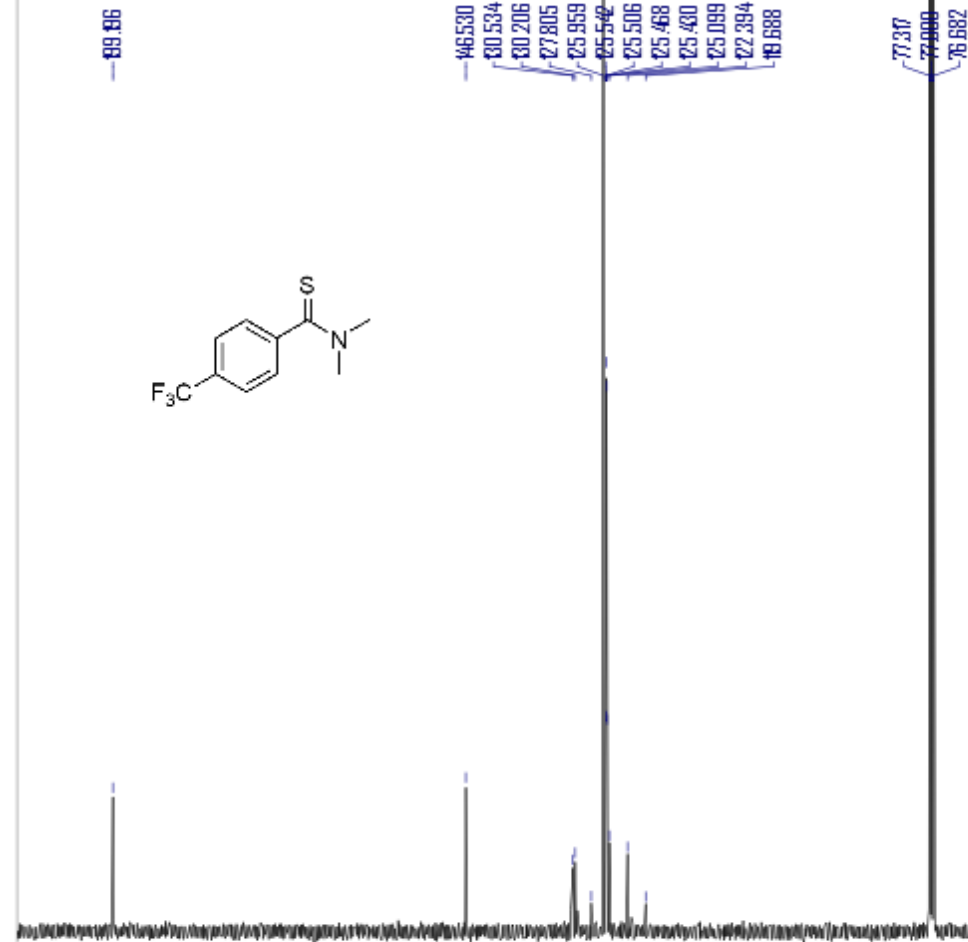

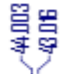

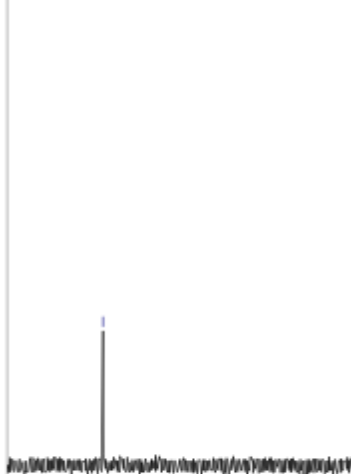

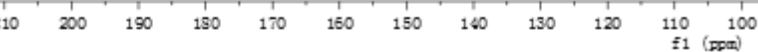

so

60

$40 \quad 30 \quad 20 \quad 10 \quad 0$ 
器
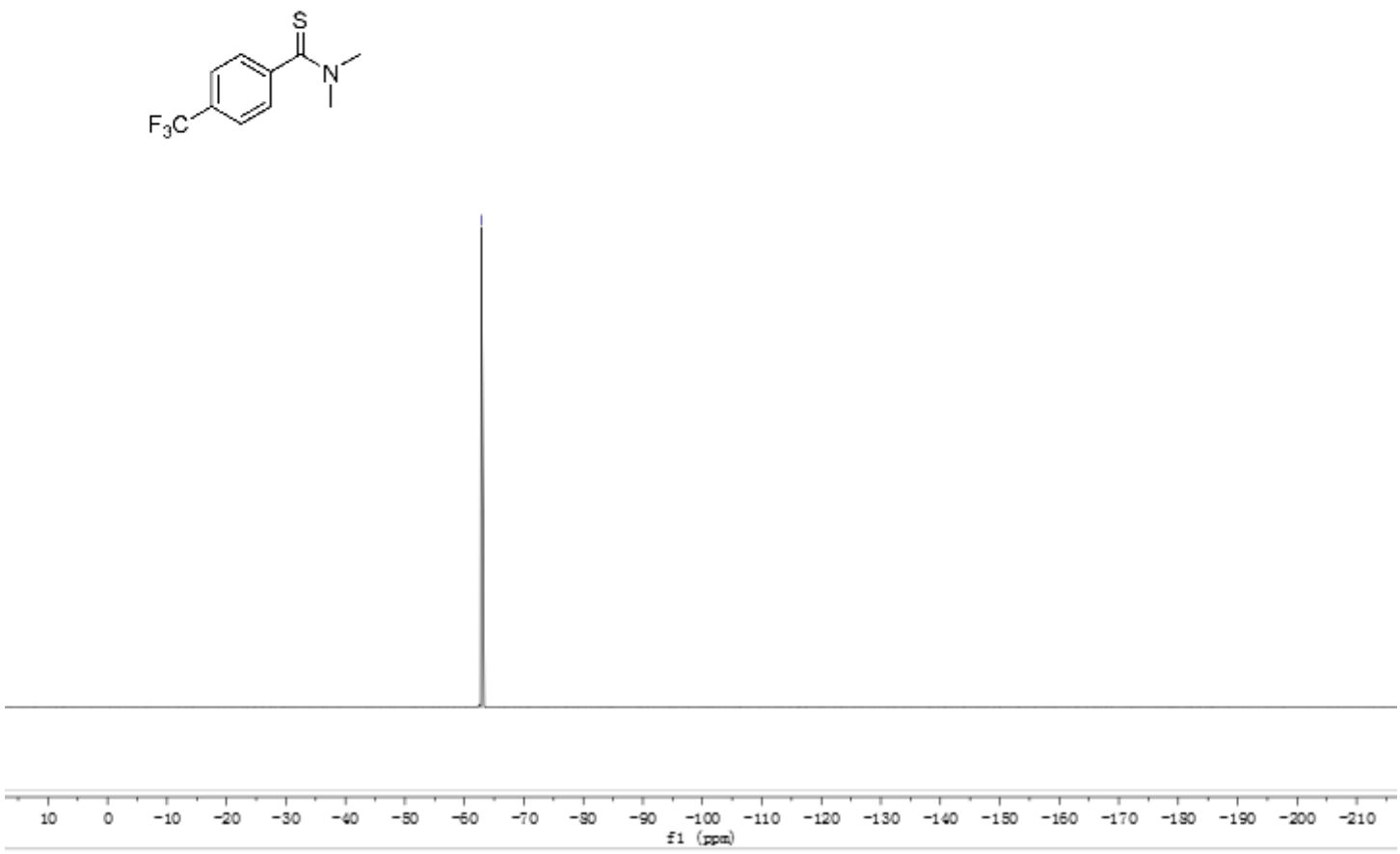

3u:

운론

嵒留
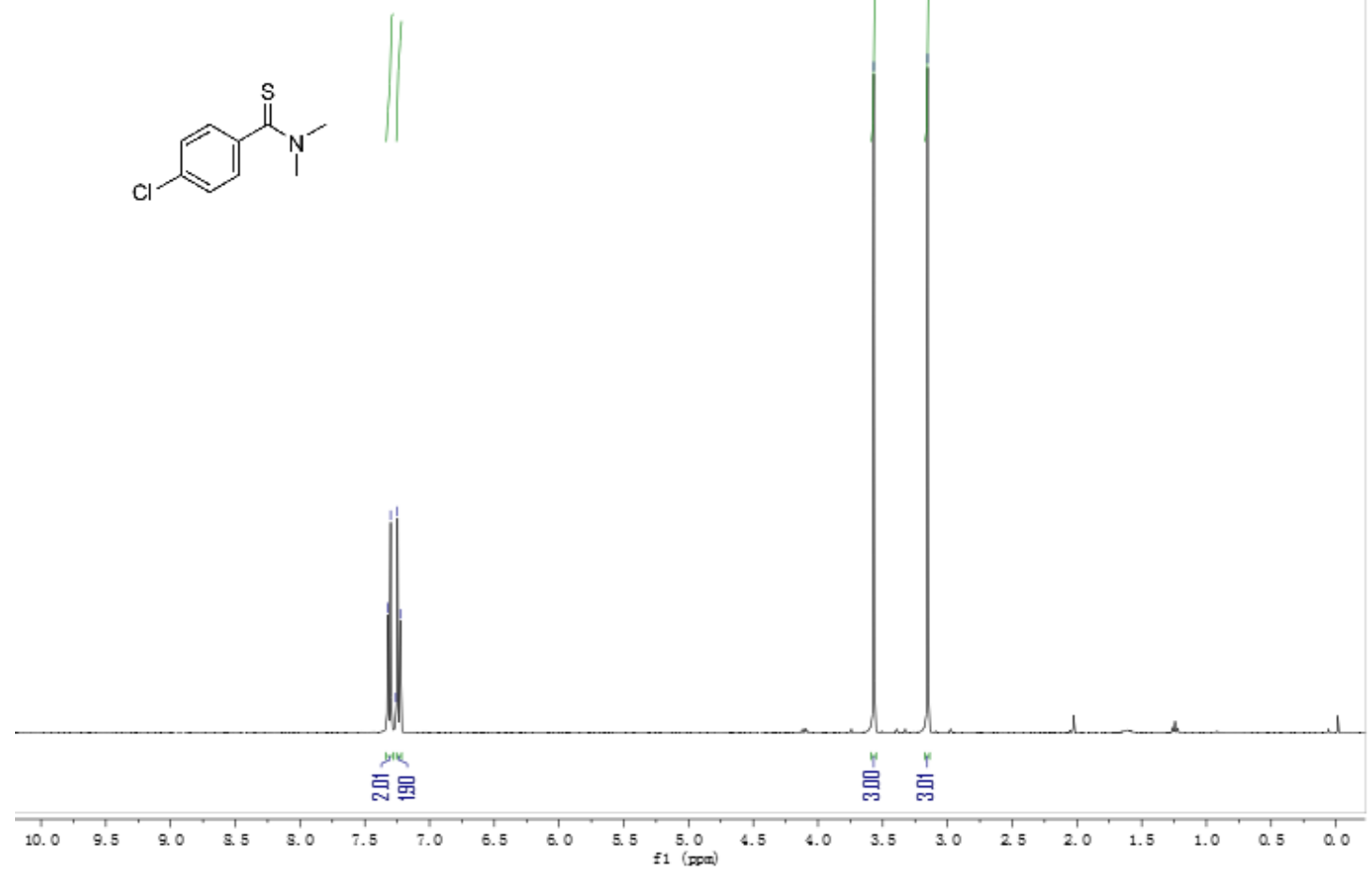

66 


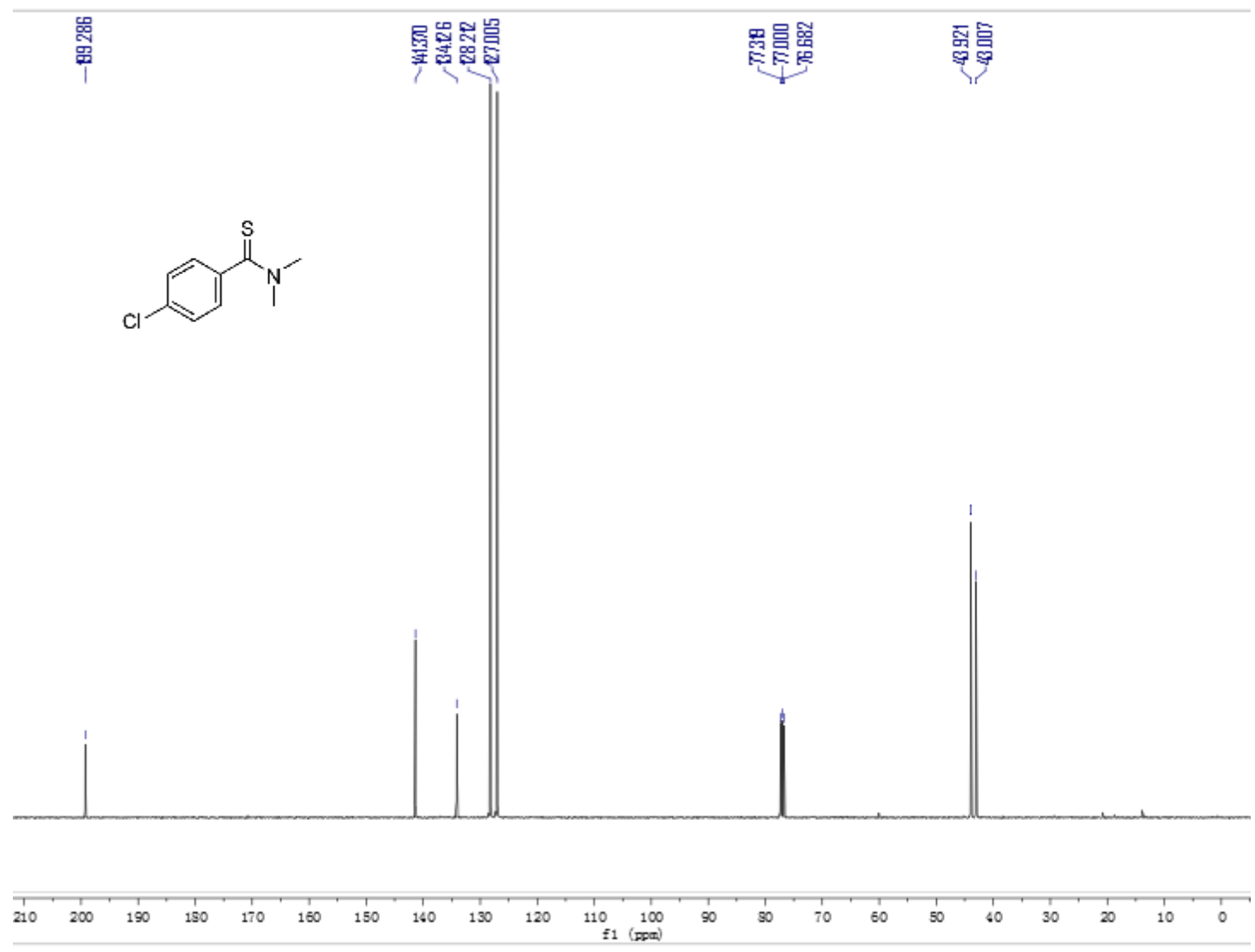

3v:

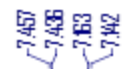

嶨<smiles>CN(C)C(=S)c1ccc(Br)cc1</smiles>
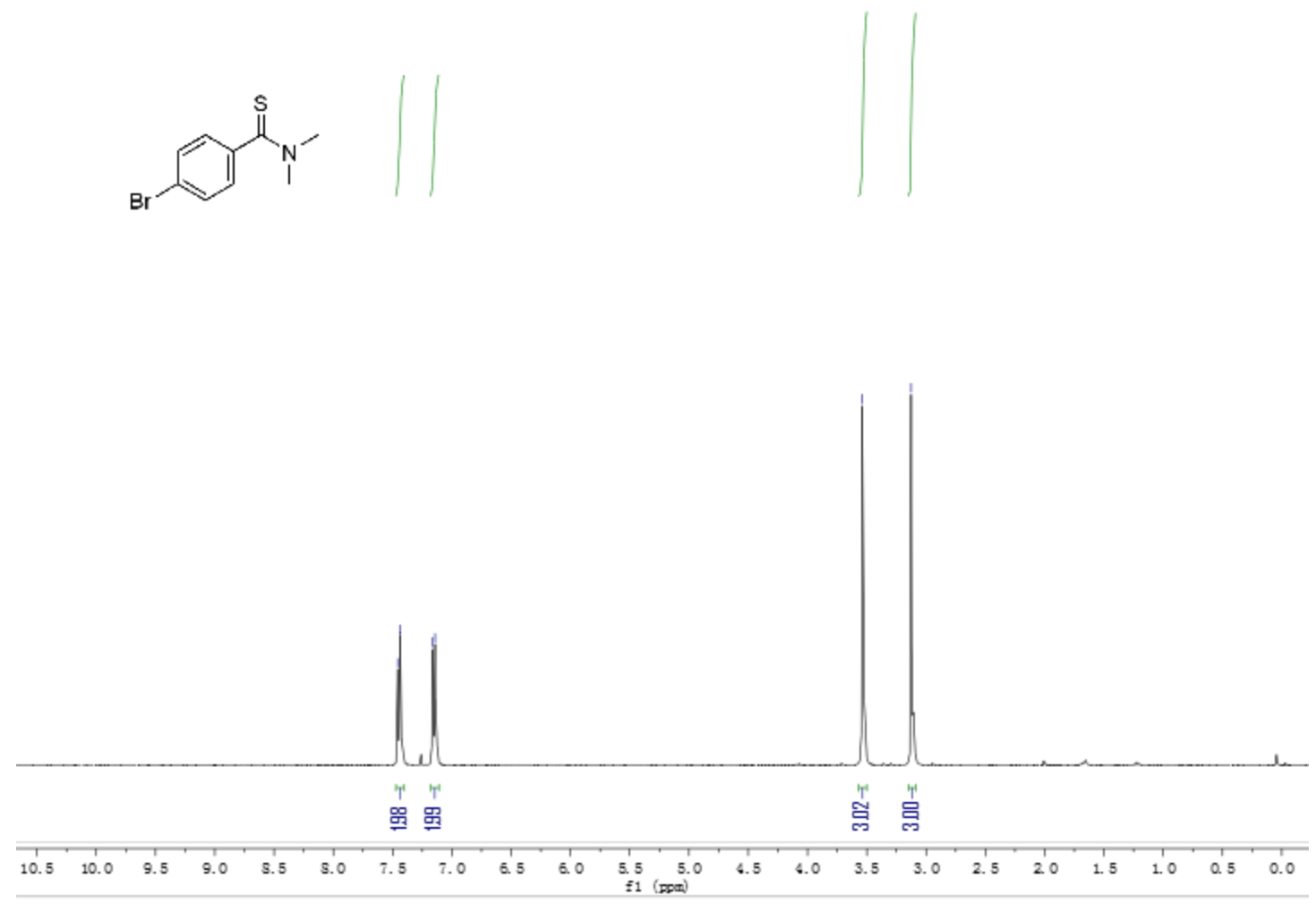


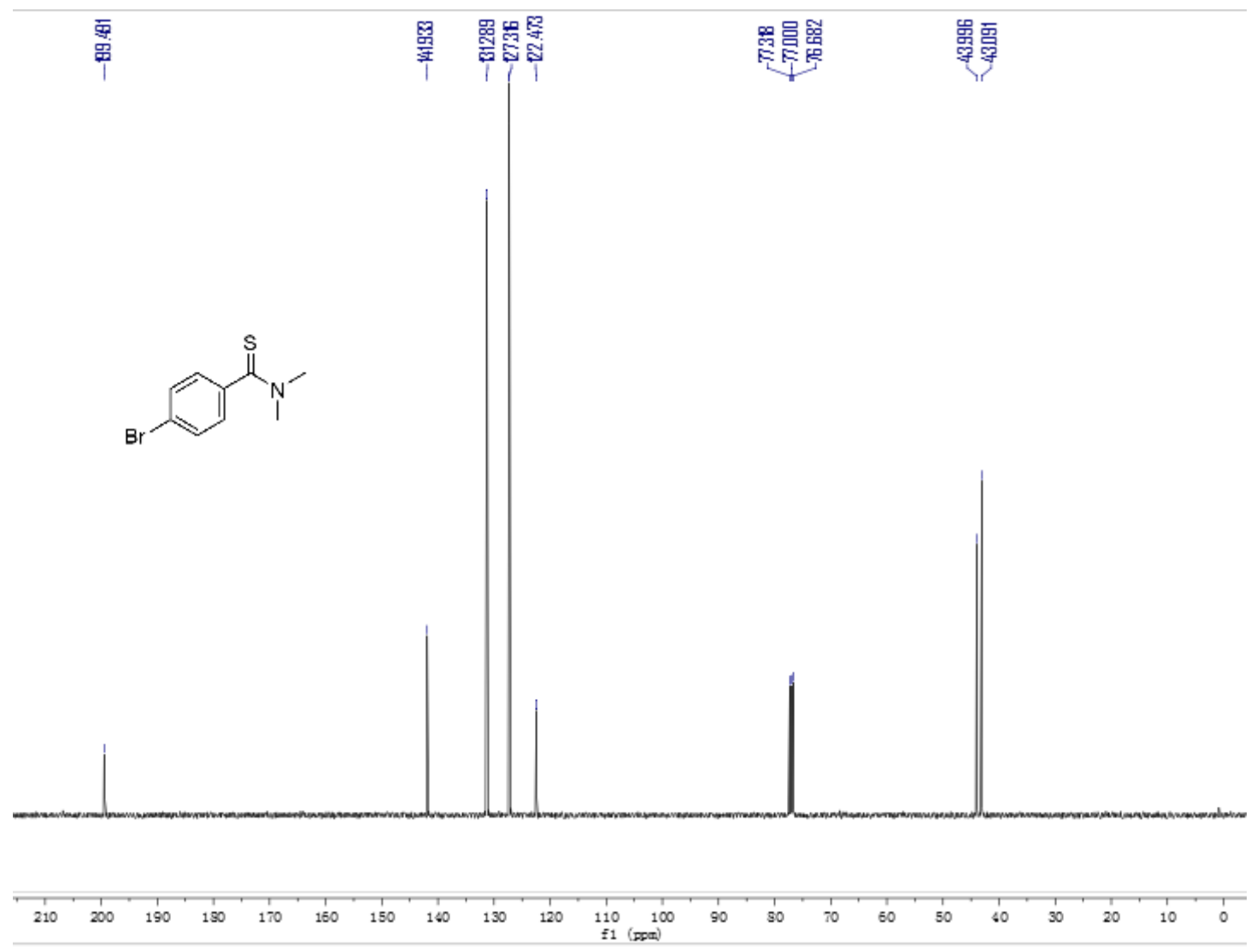

3w:

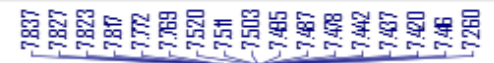

䔽 总

$\mathbb{N}^{1}$

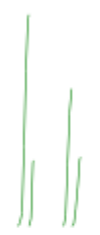

$\mid$

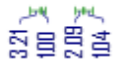

芦曾

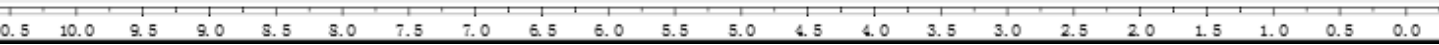



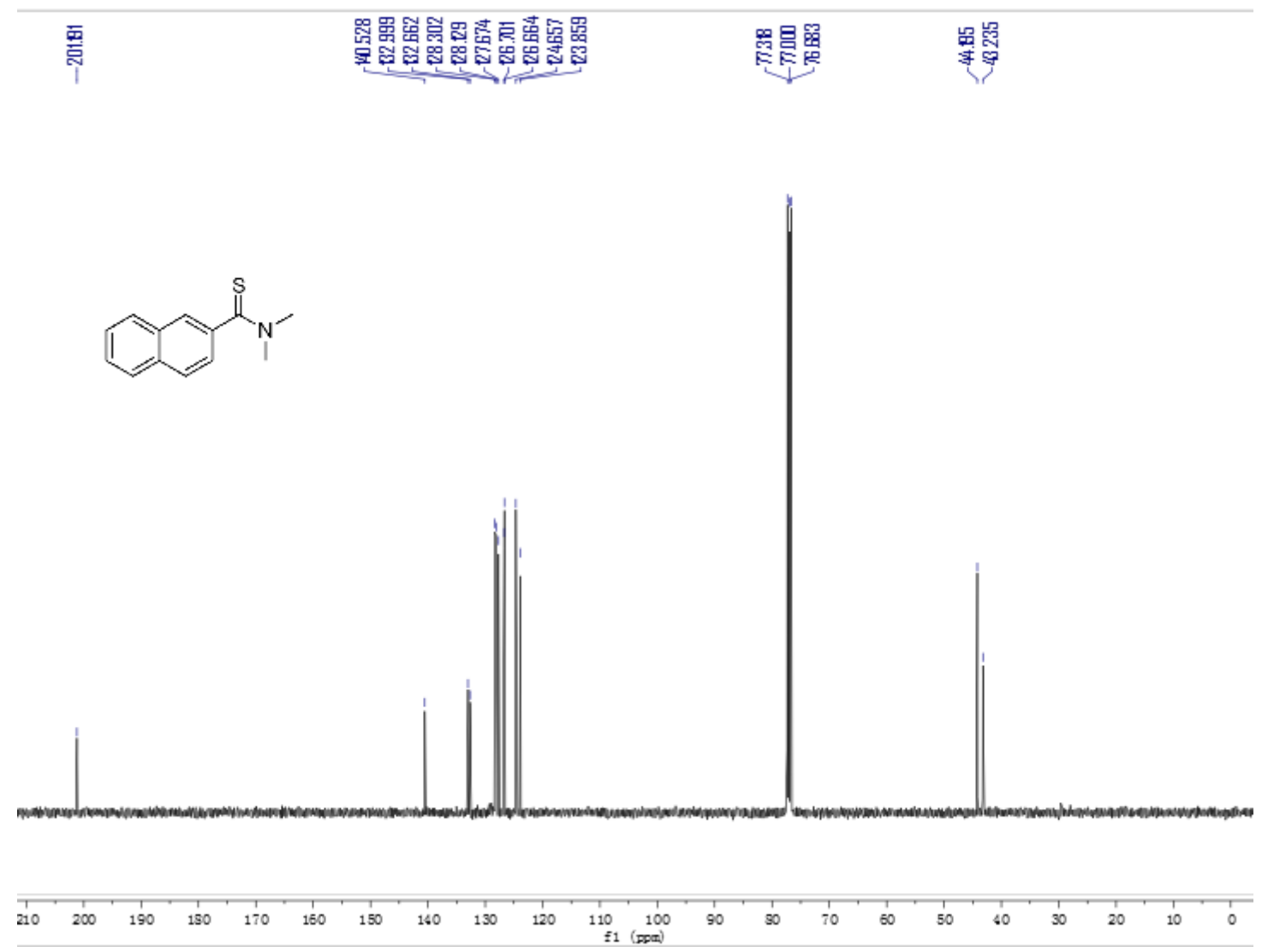

3x:
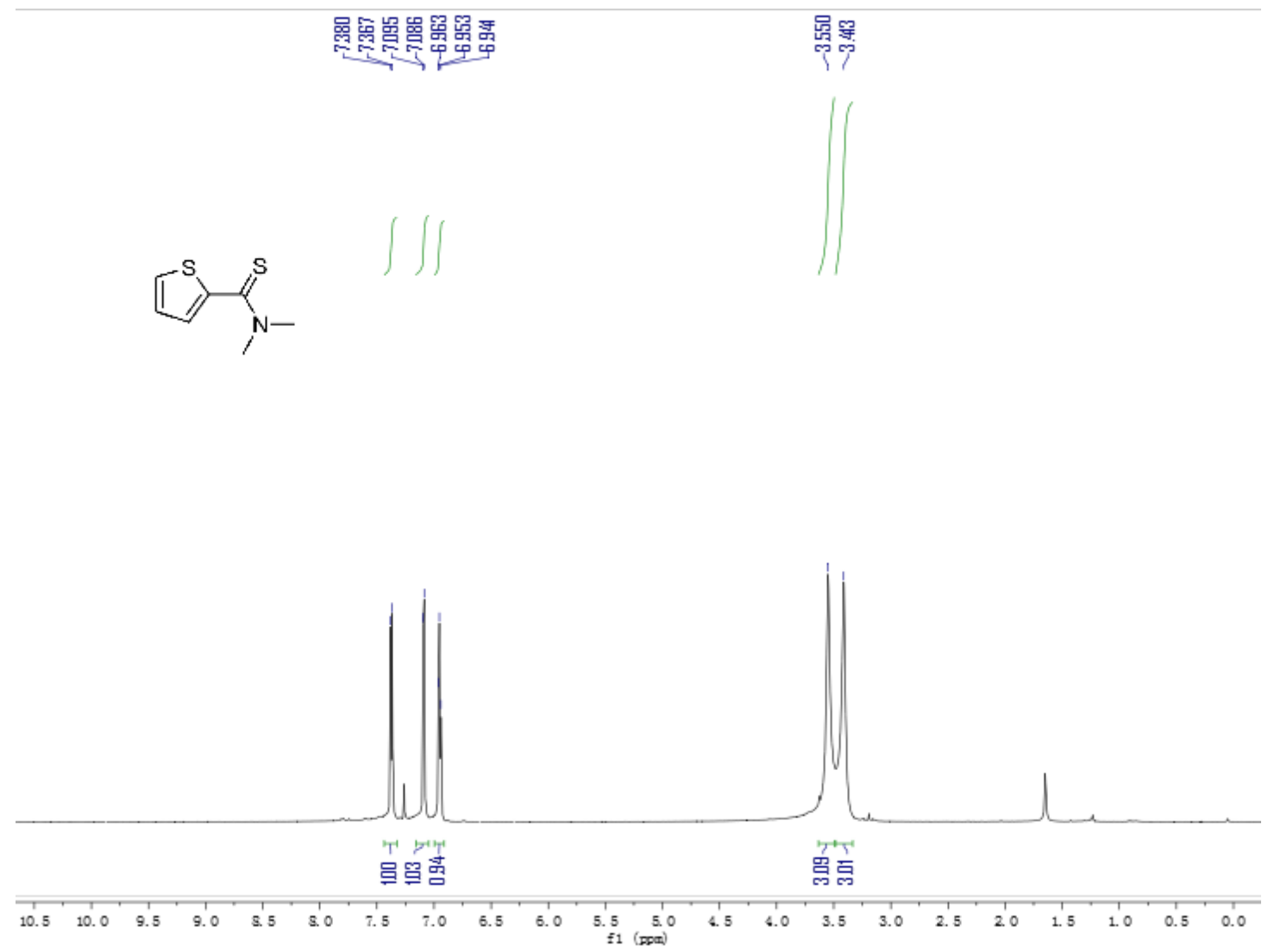


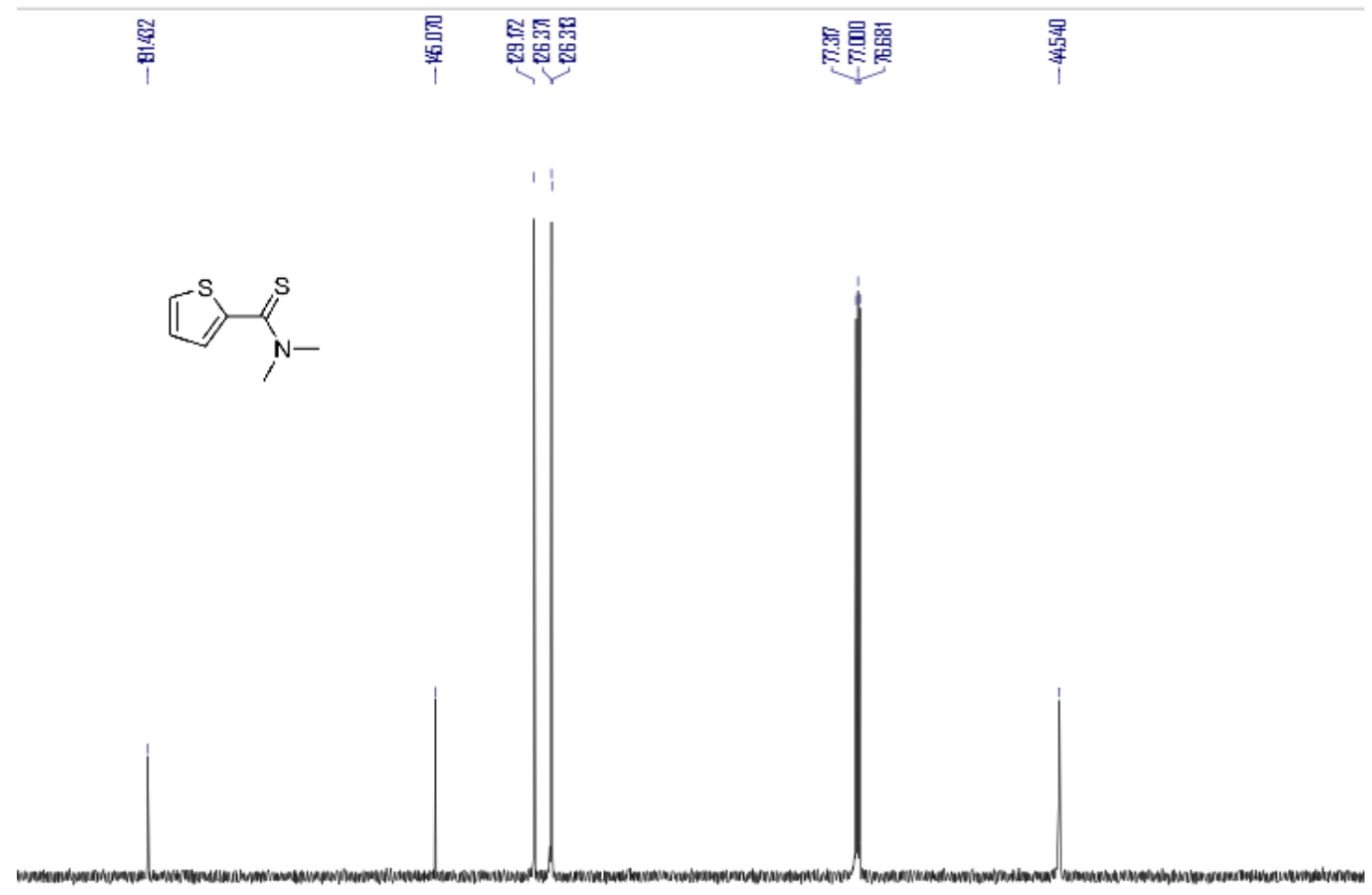

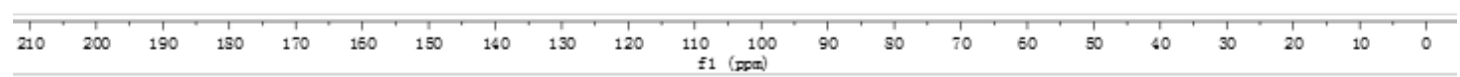

3y:

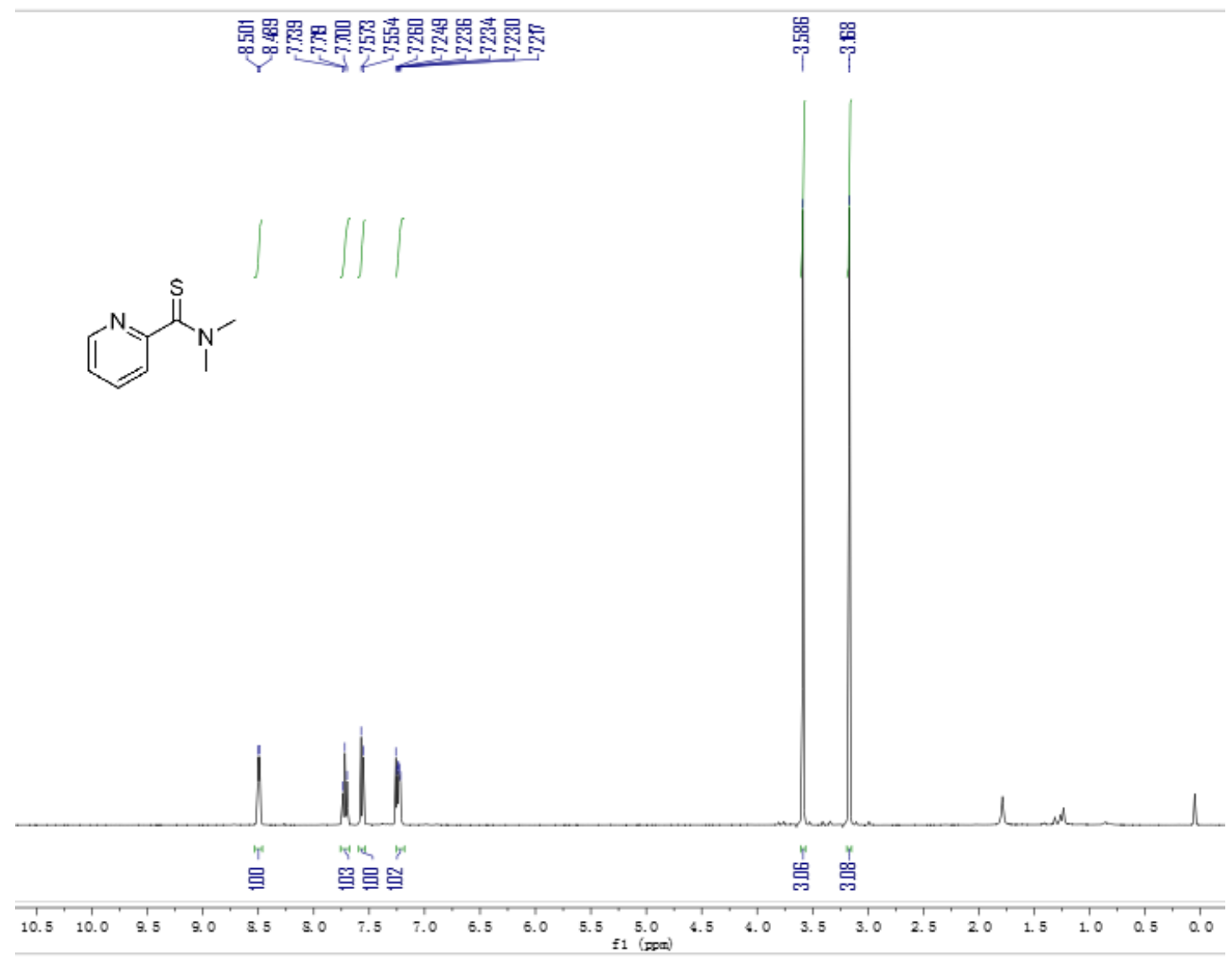




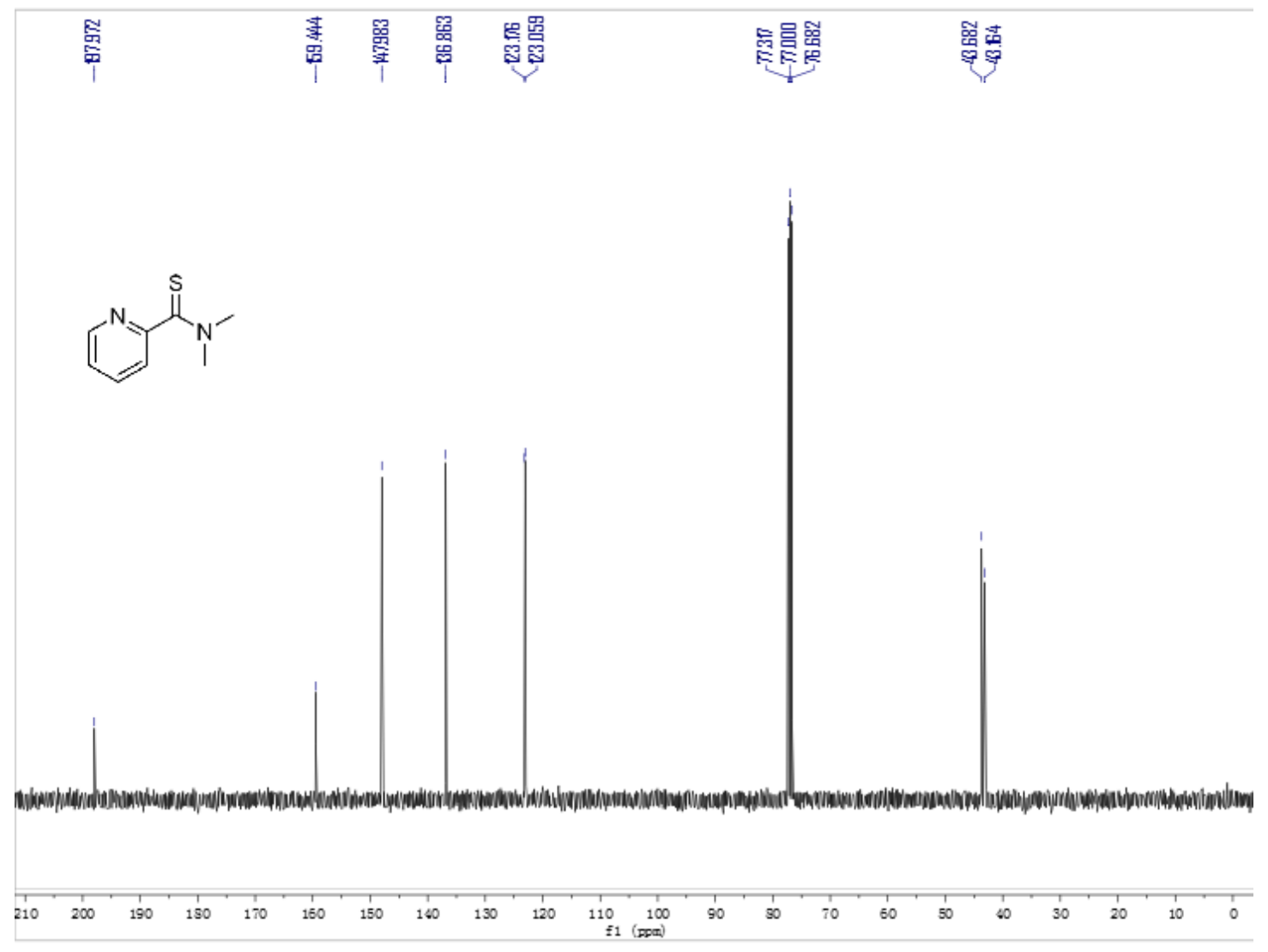

3z:

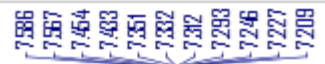

㩕帮

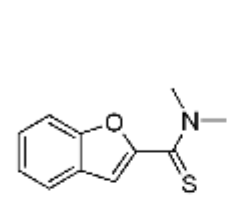

\|\|$\|$
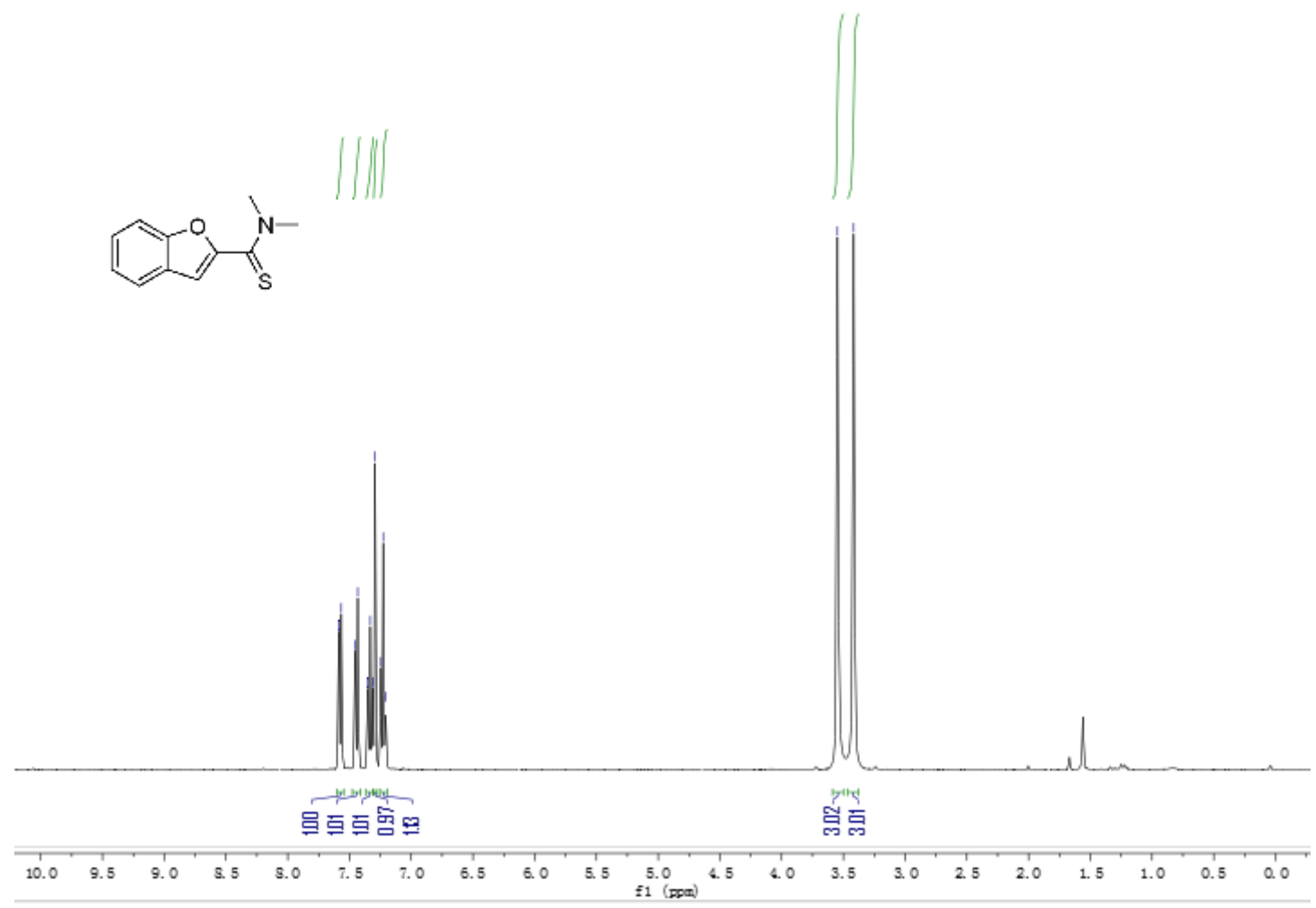

71 
声

品監

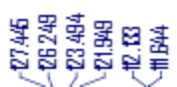

器袁吡

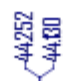
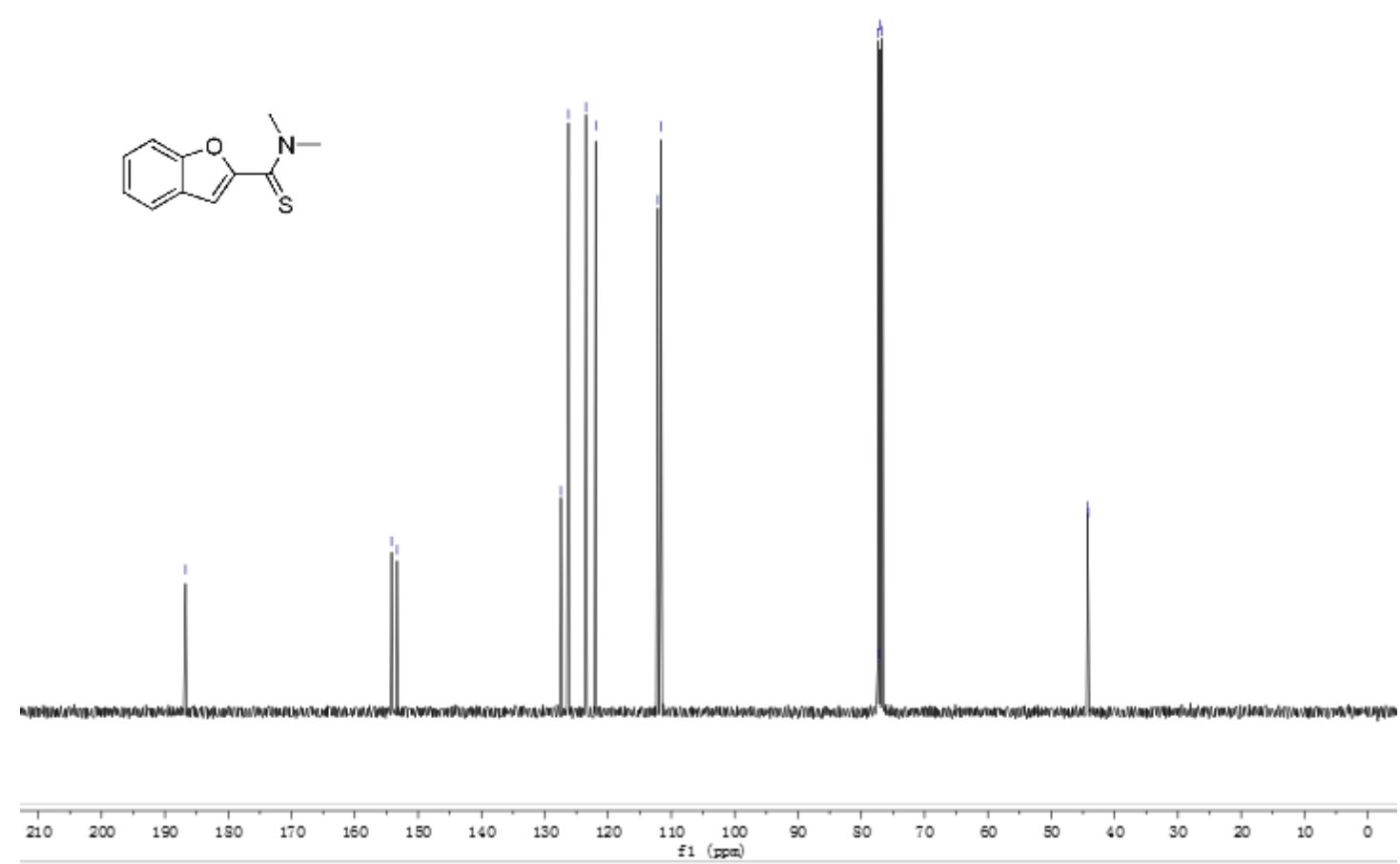

3aa:

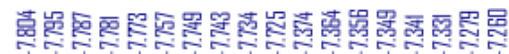

哭郘
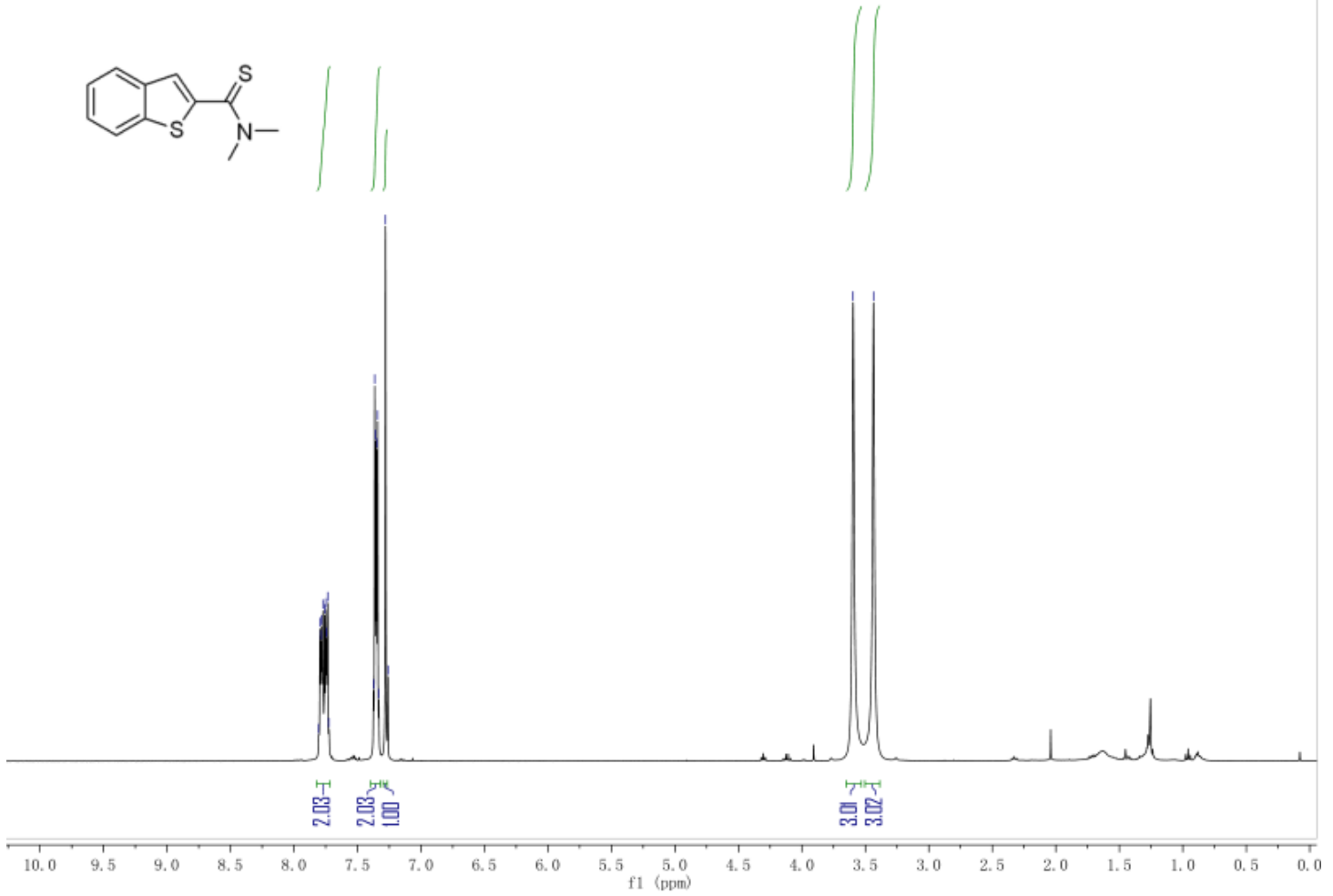

72 

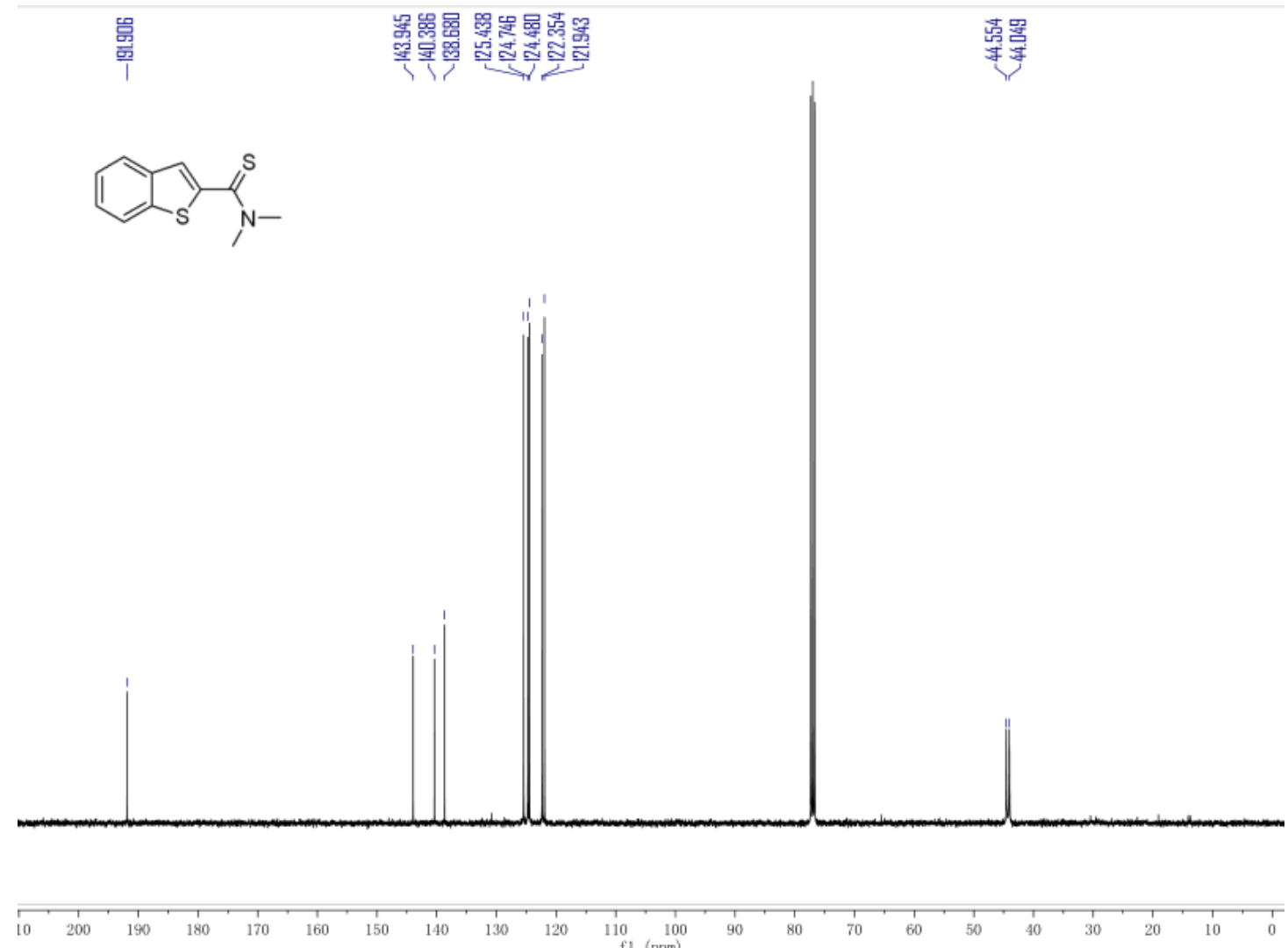

3ab:

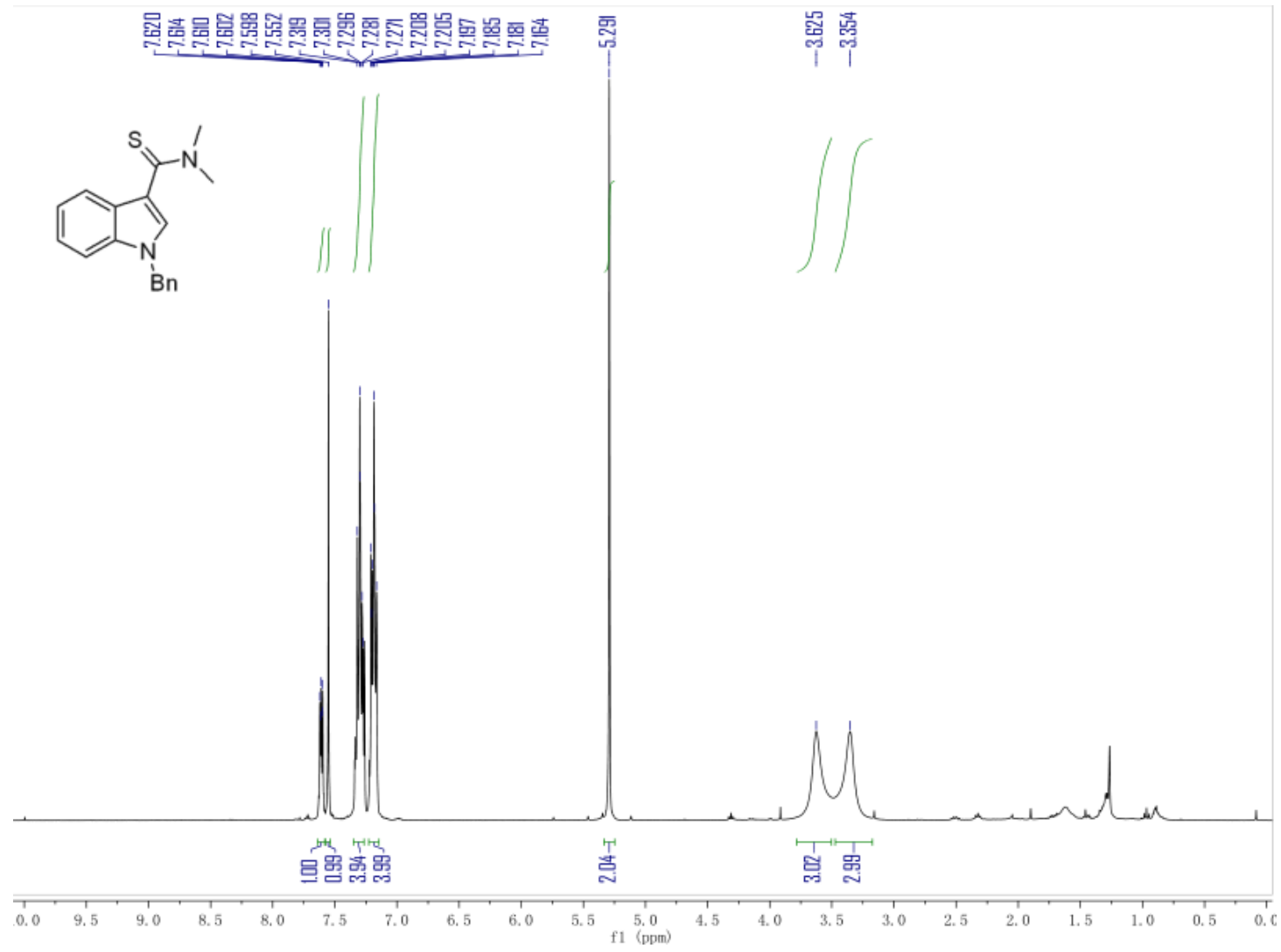




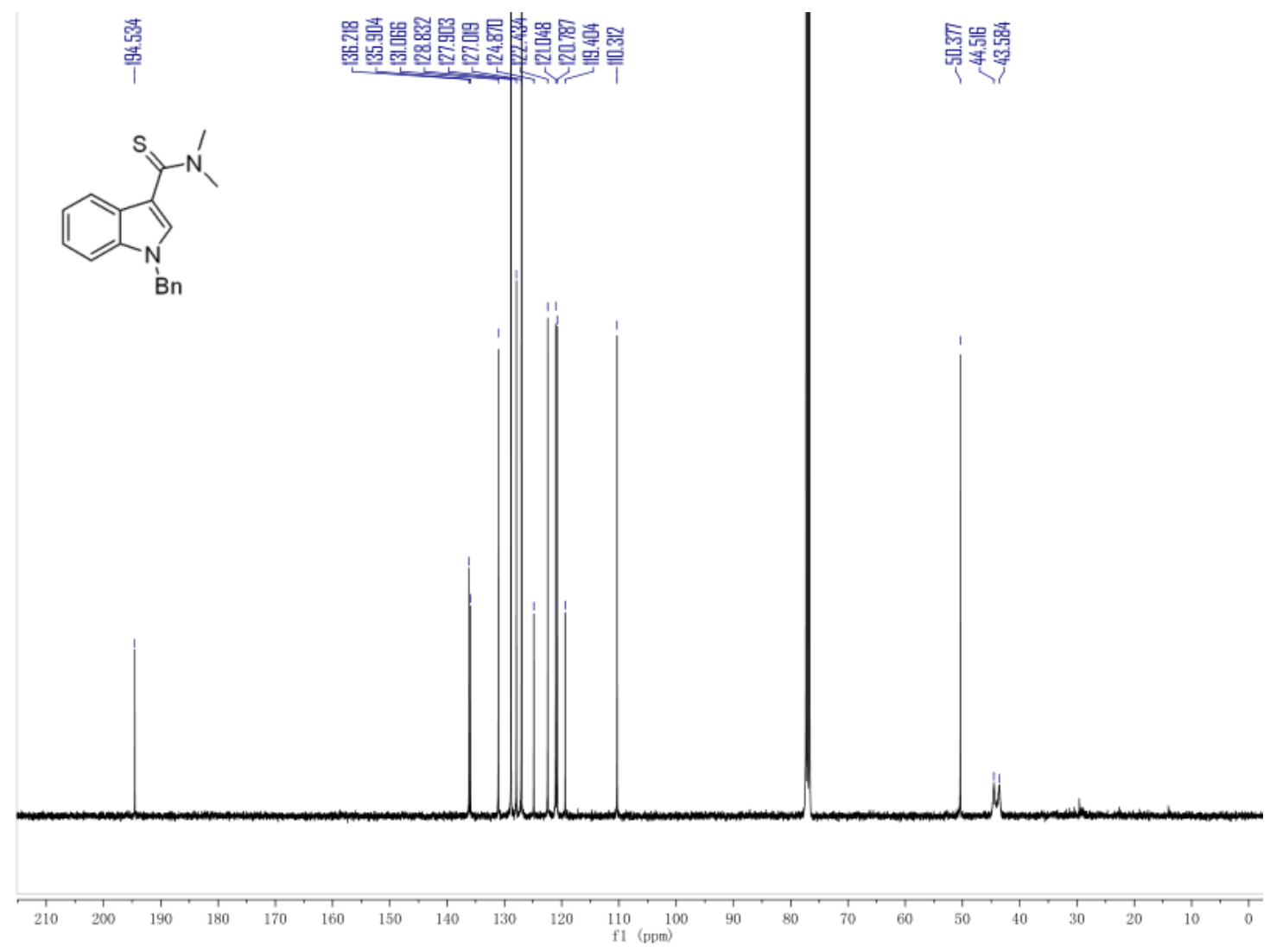

3ac:

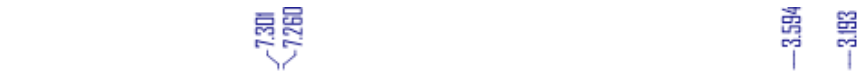<smiles>CN(C)C(=S)c1ccc(C(=S)N(C)C)cc1</smiles>

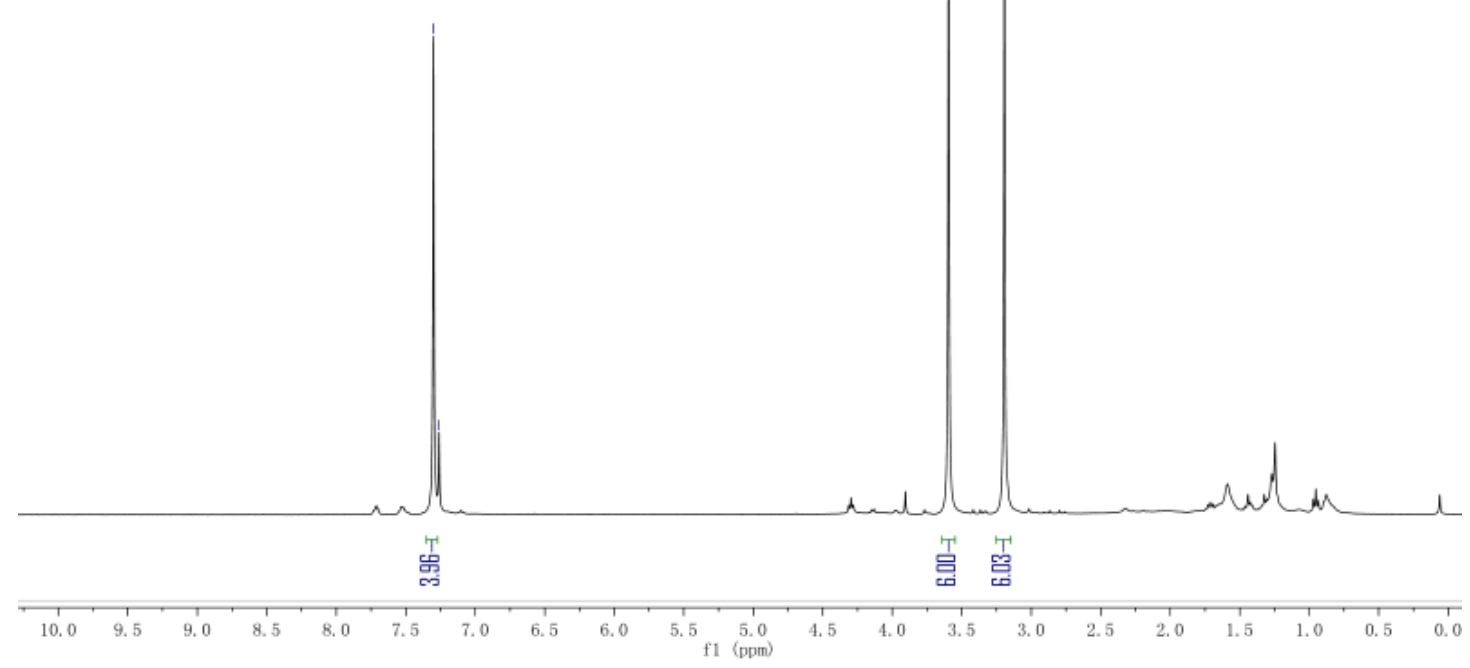




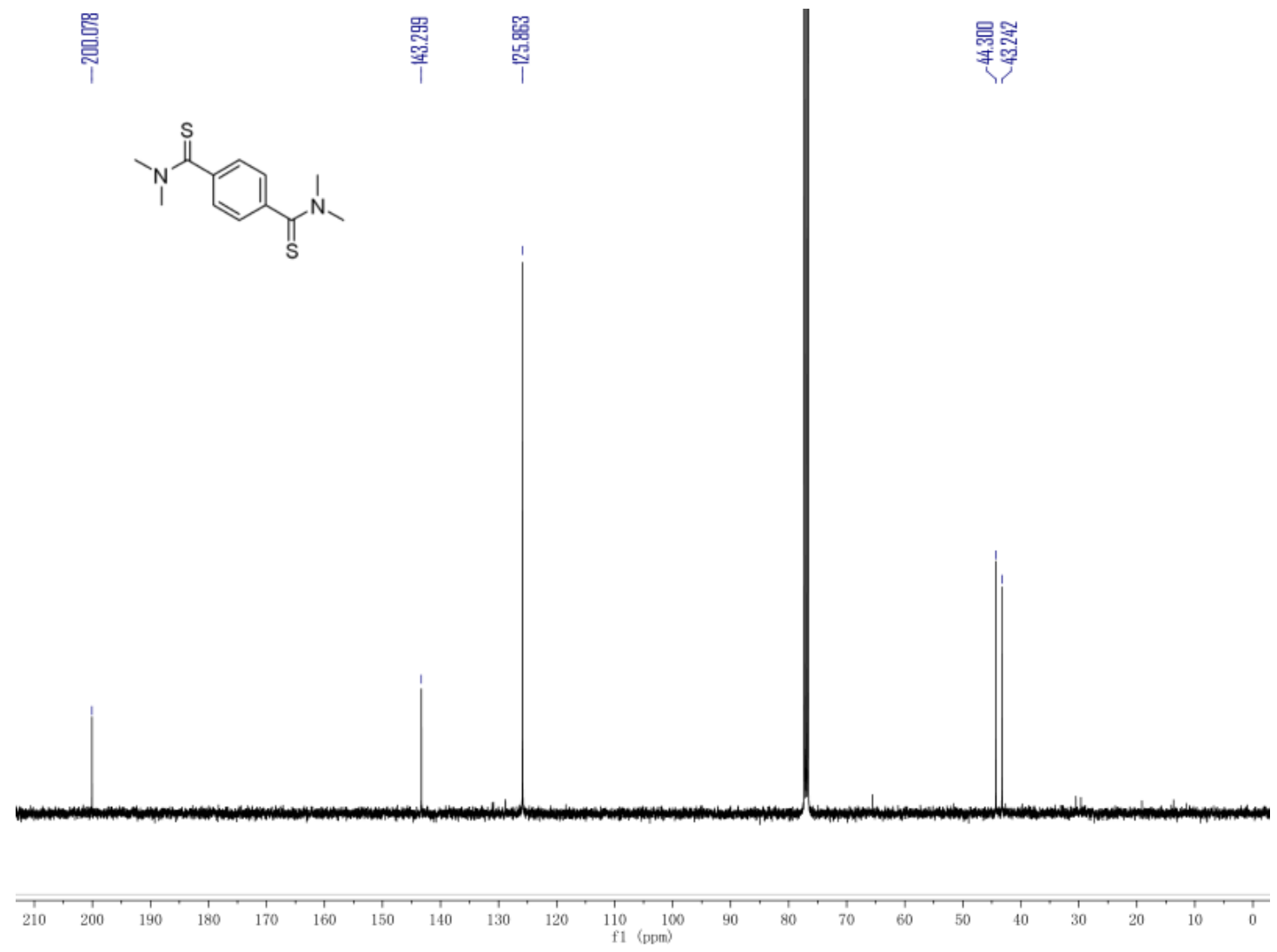

3ad:

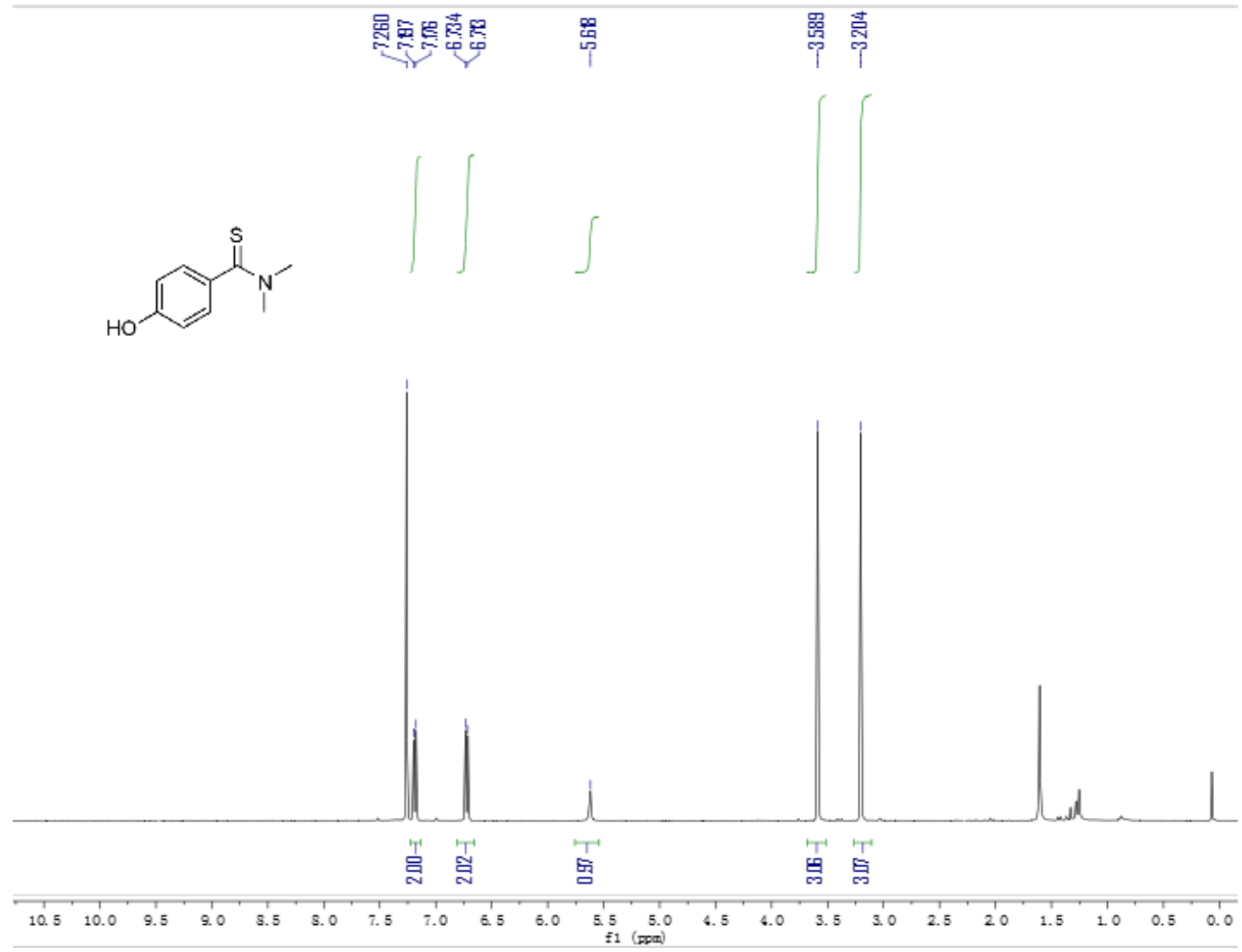



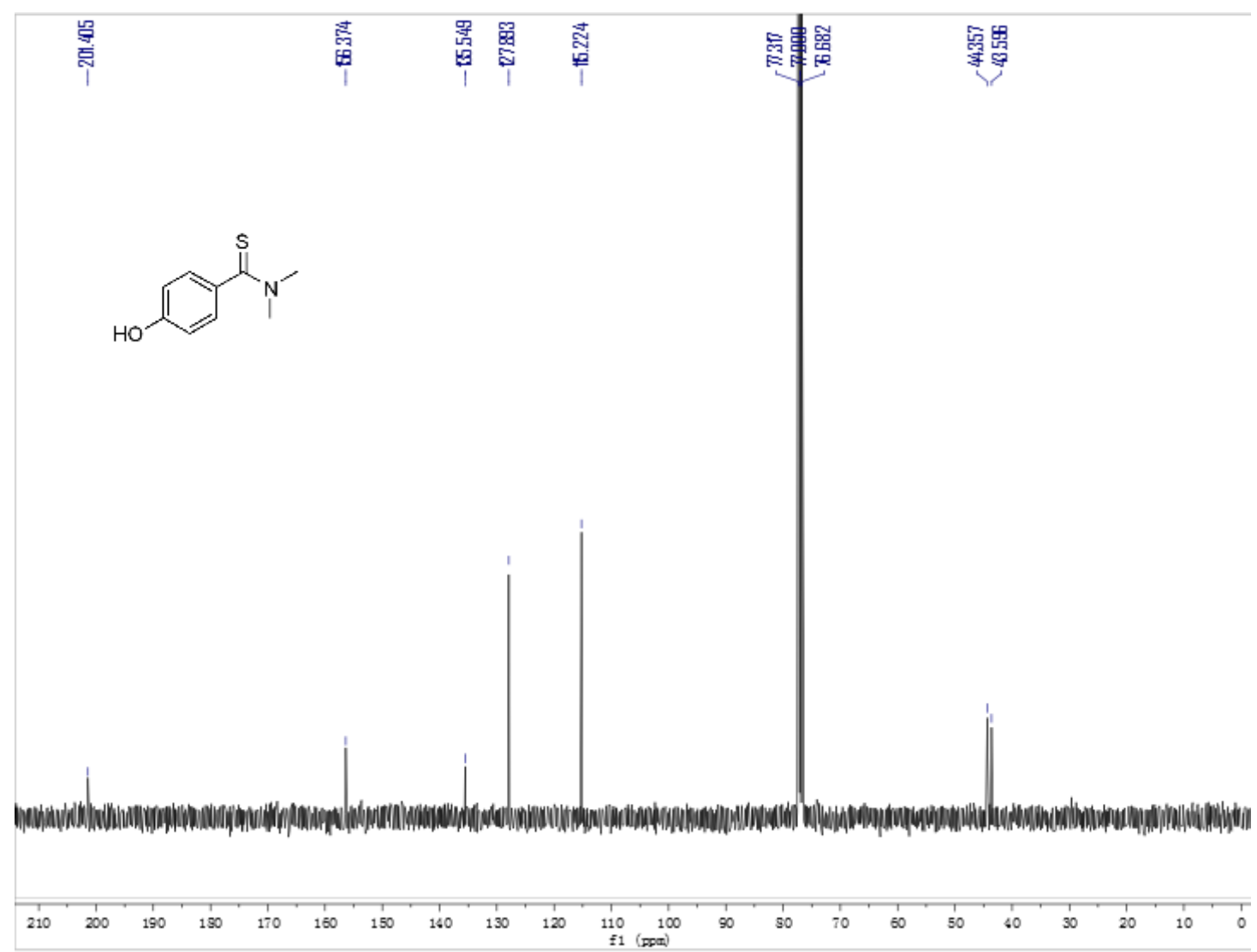

3ae:

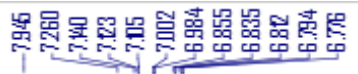
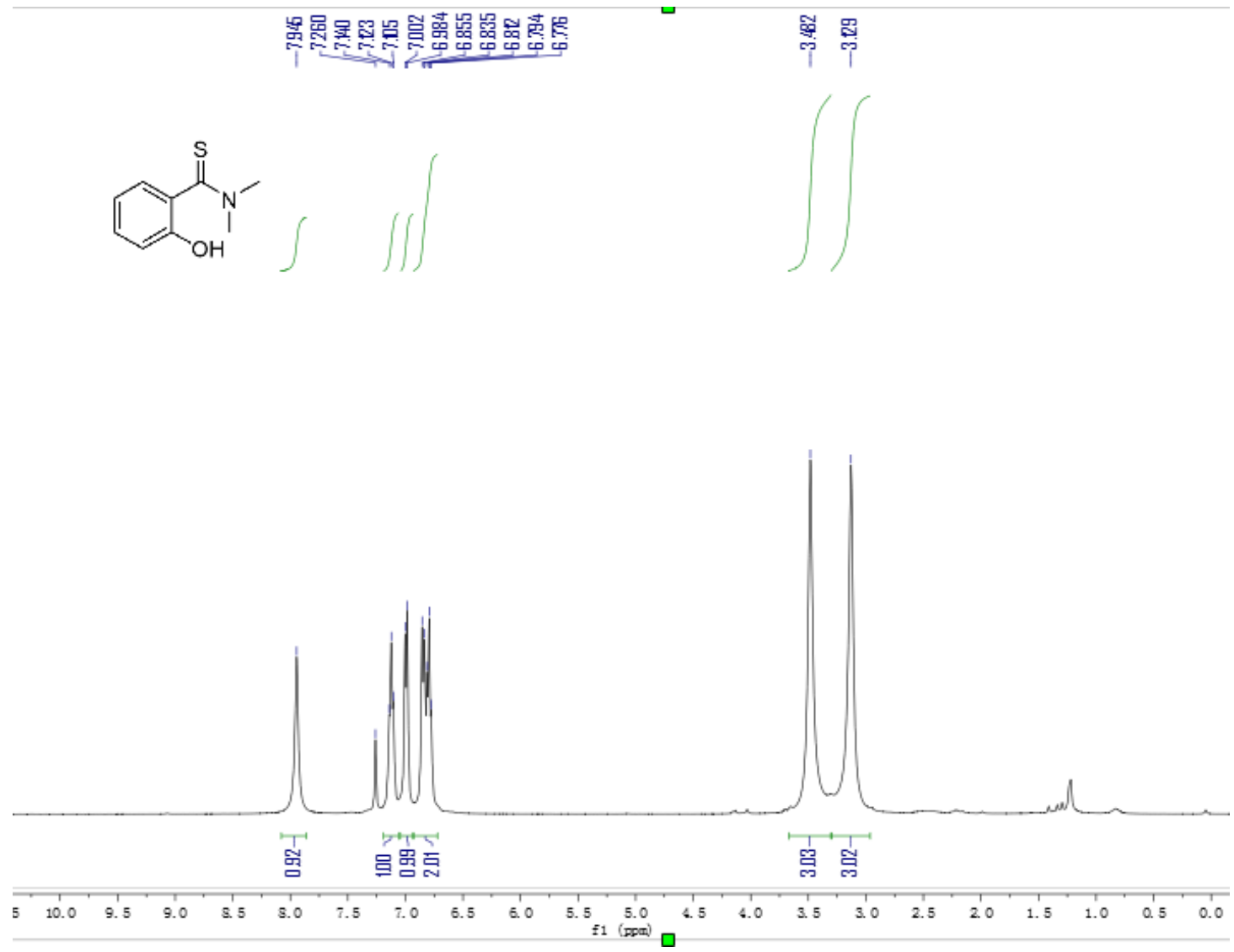

76 


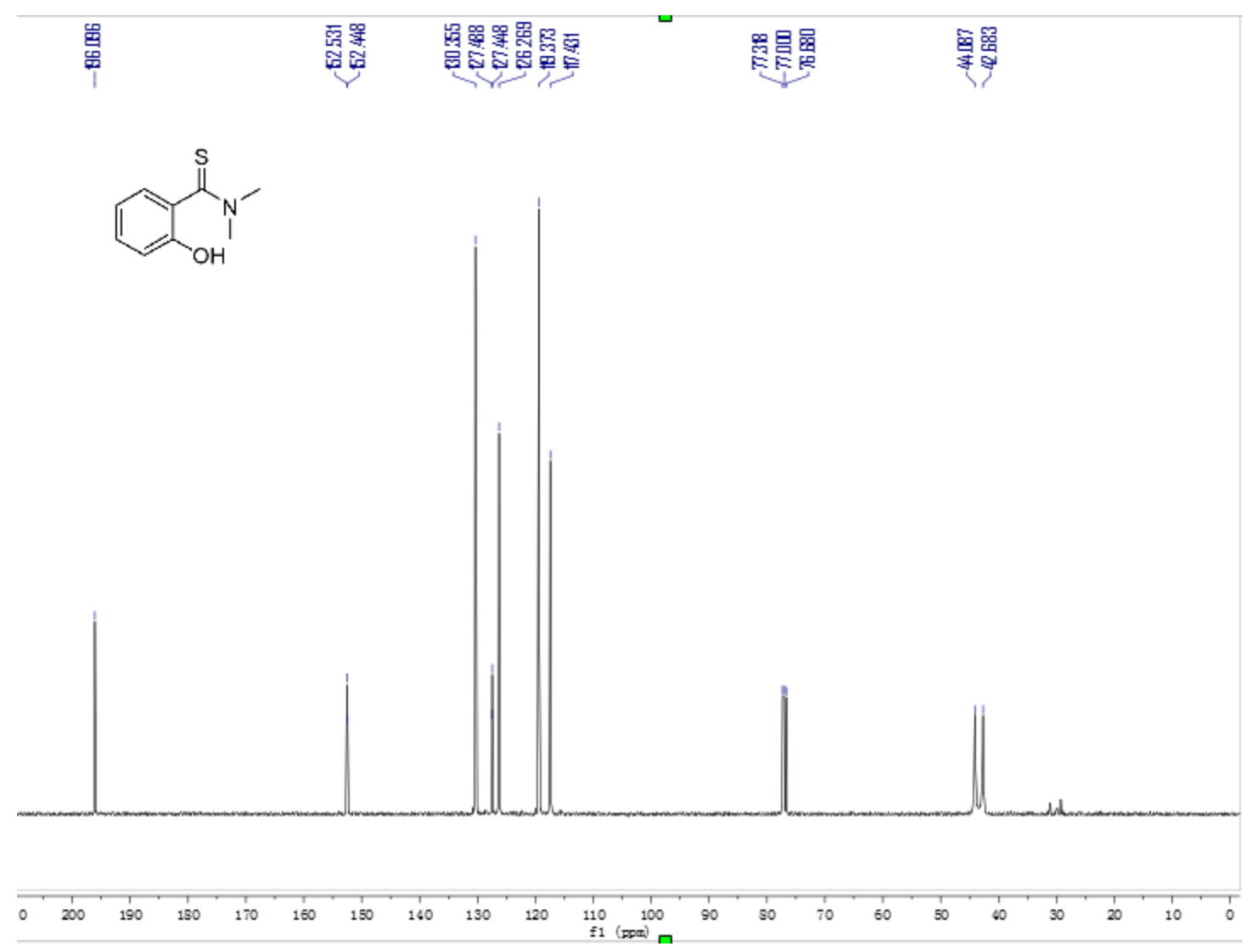

3af:

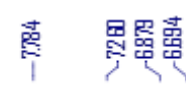

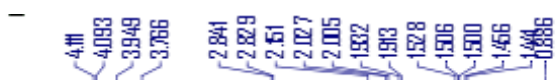
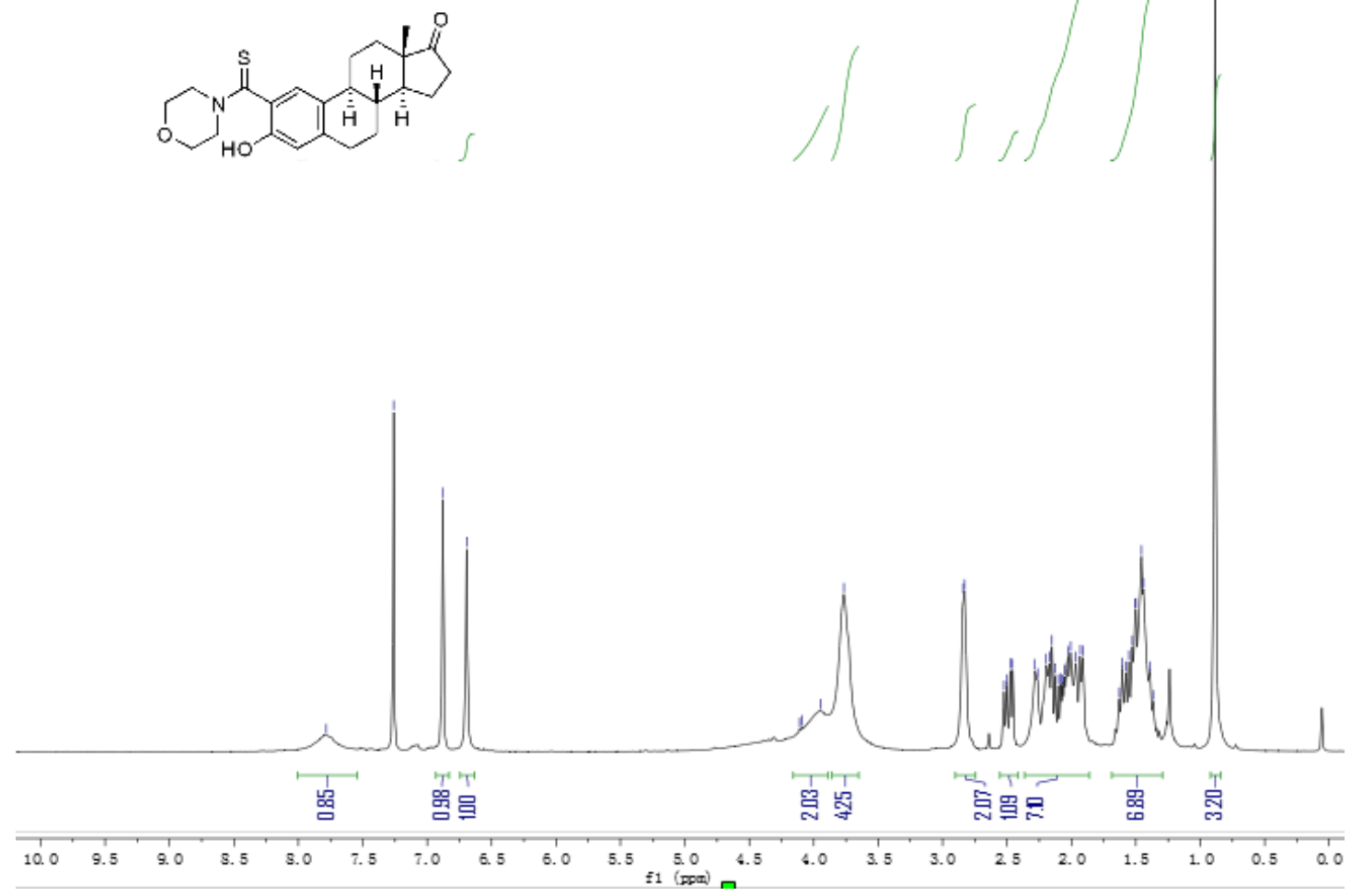
苞毒
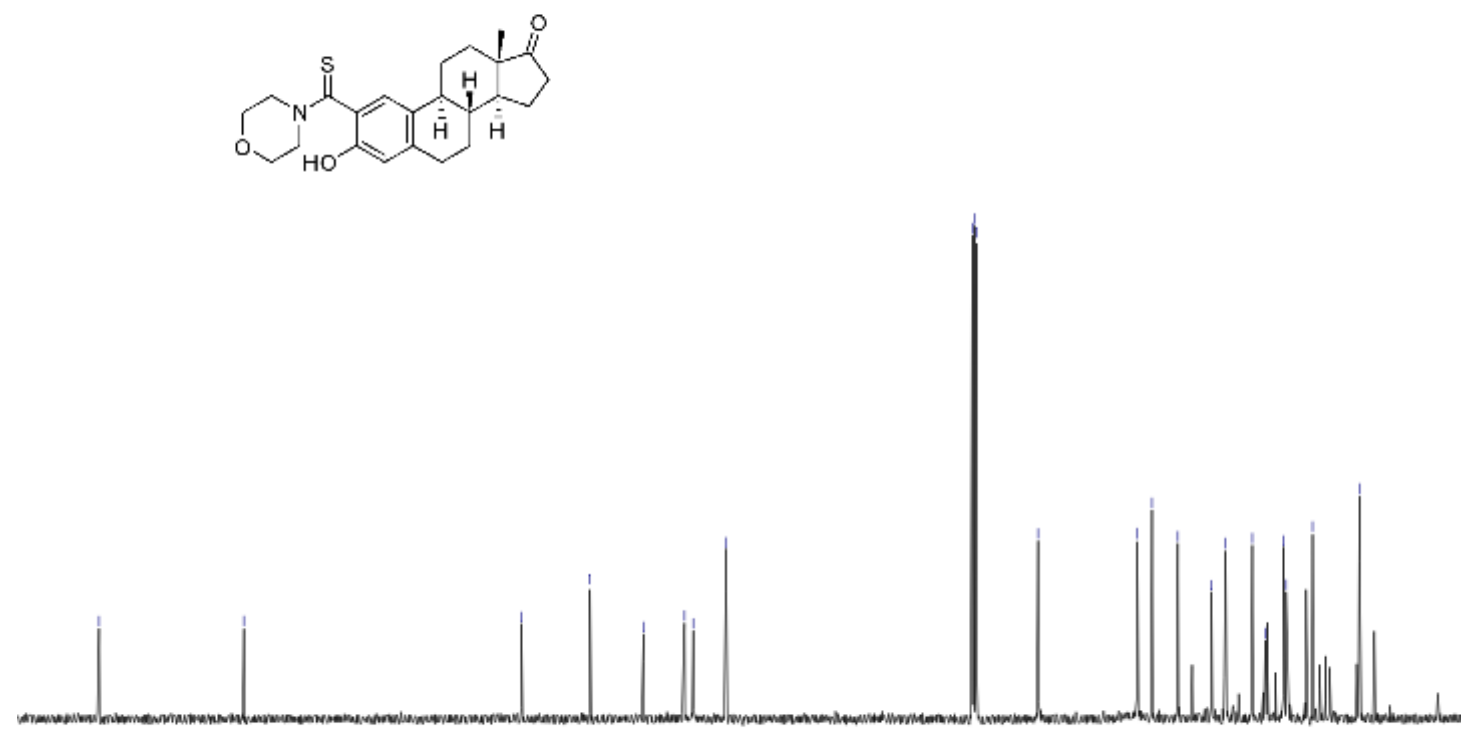

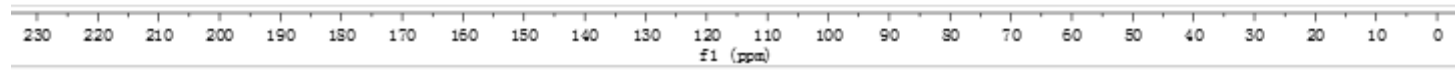

4aa:

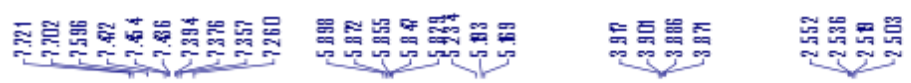
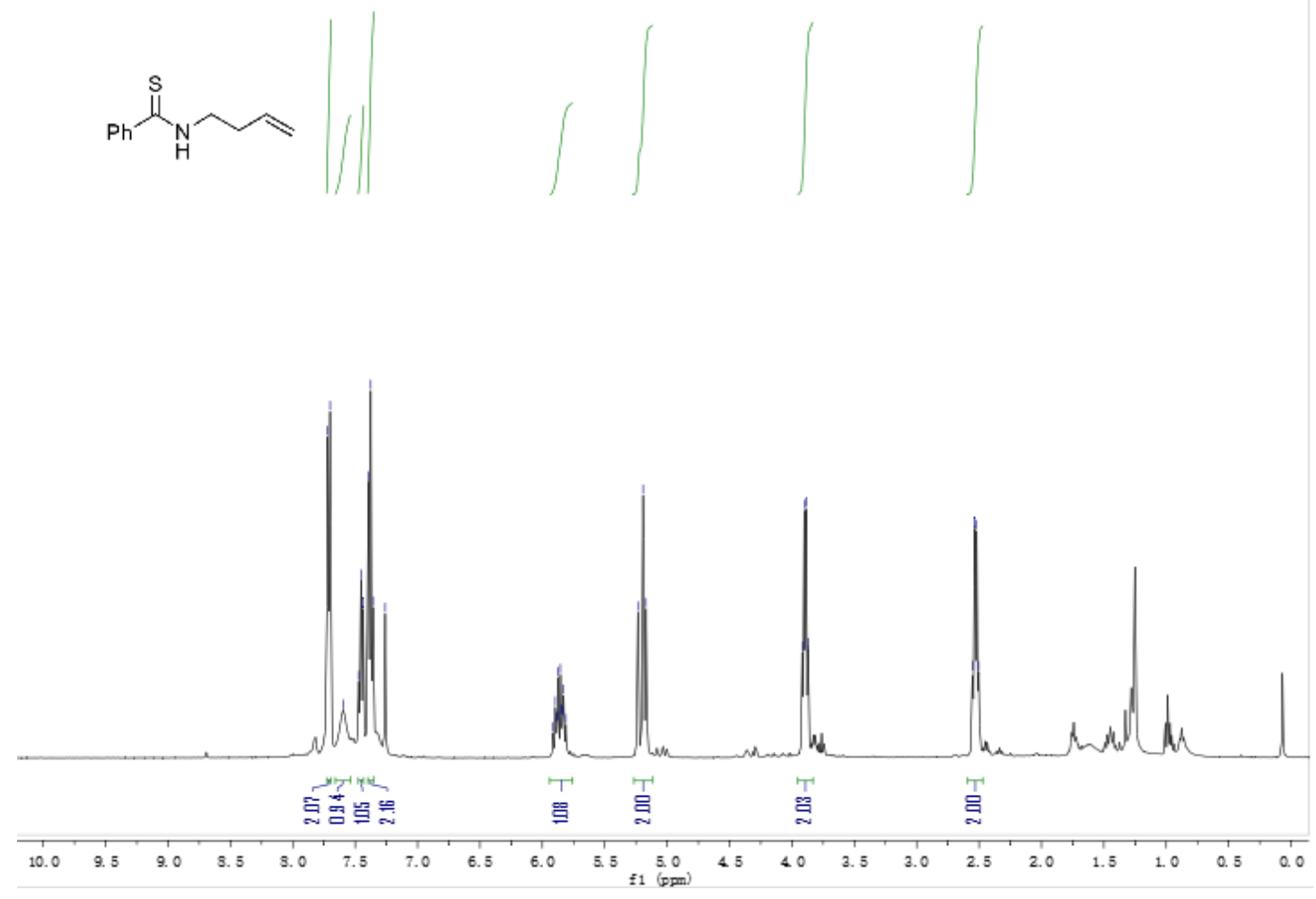


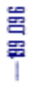<smiles>C/C=C/CCNC(=S)c1ccccc1</smiles>
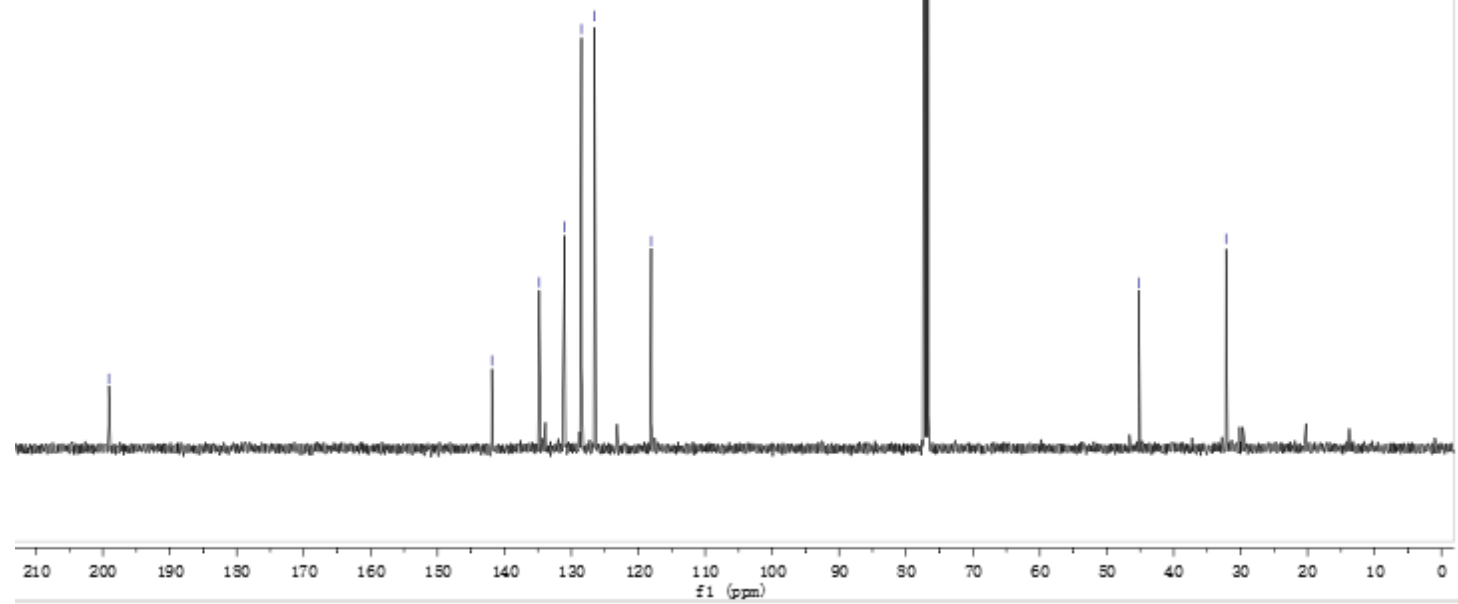

4bb:

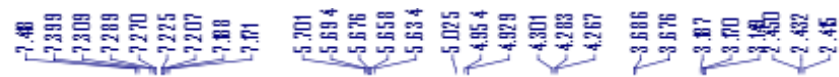<smiles>C=CCC(CCNC(=S)Nc1ccccc1)Pc1ccccc1</smiles>
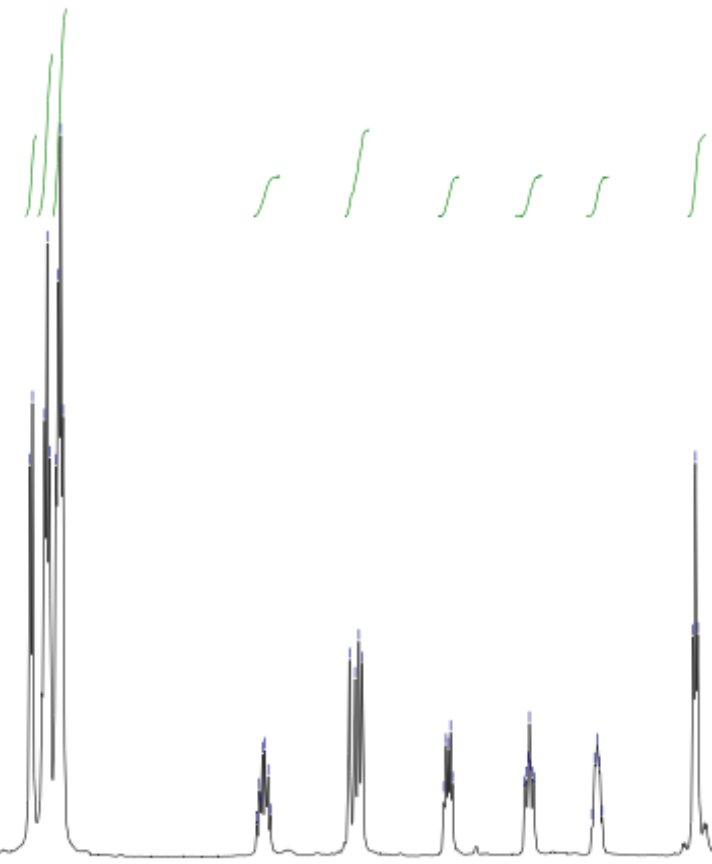

g

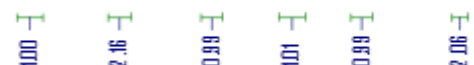

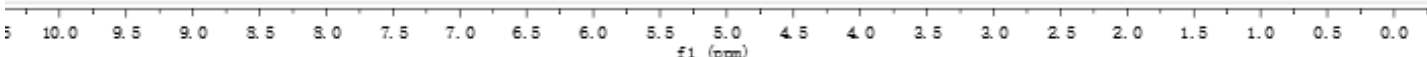




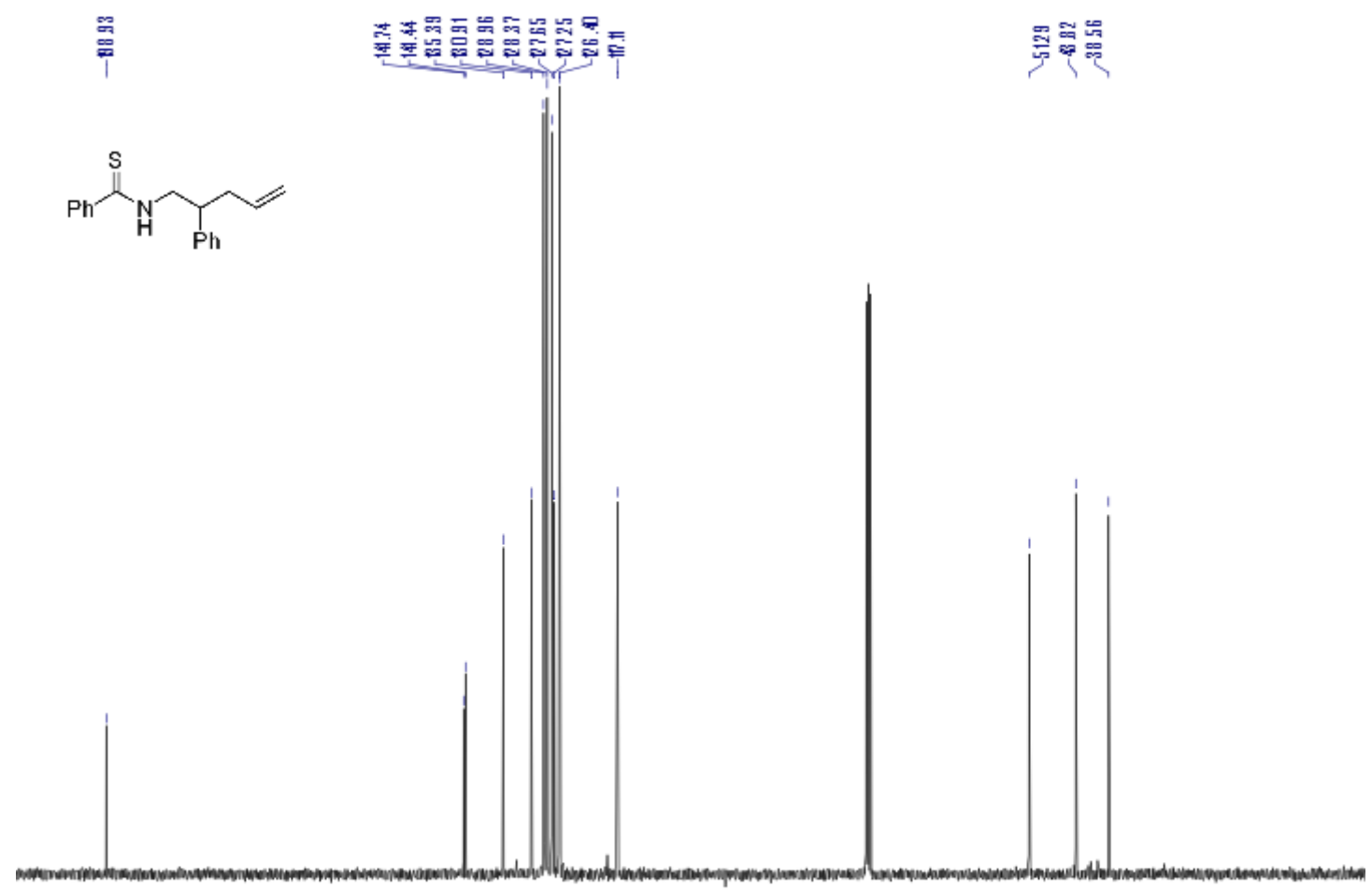

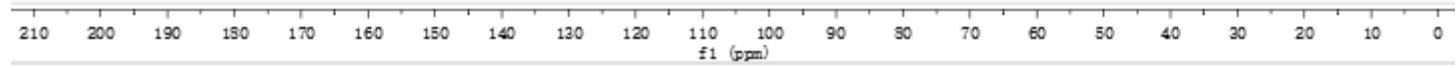

4c:

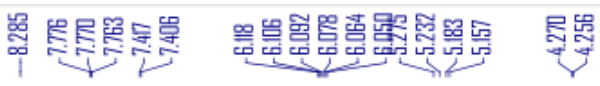
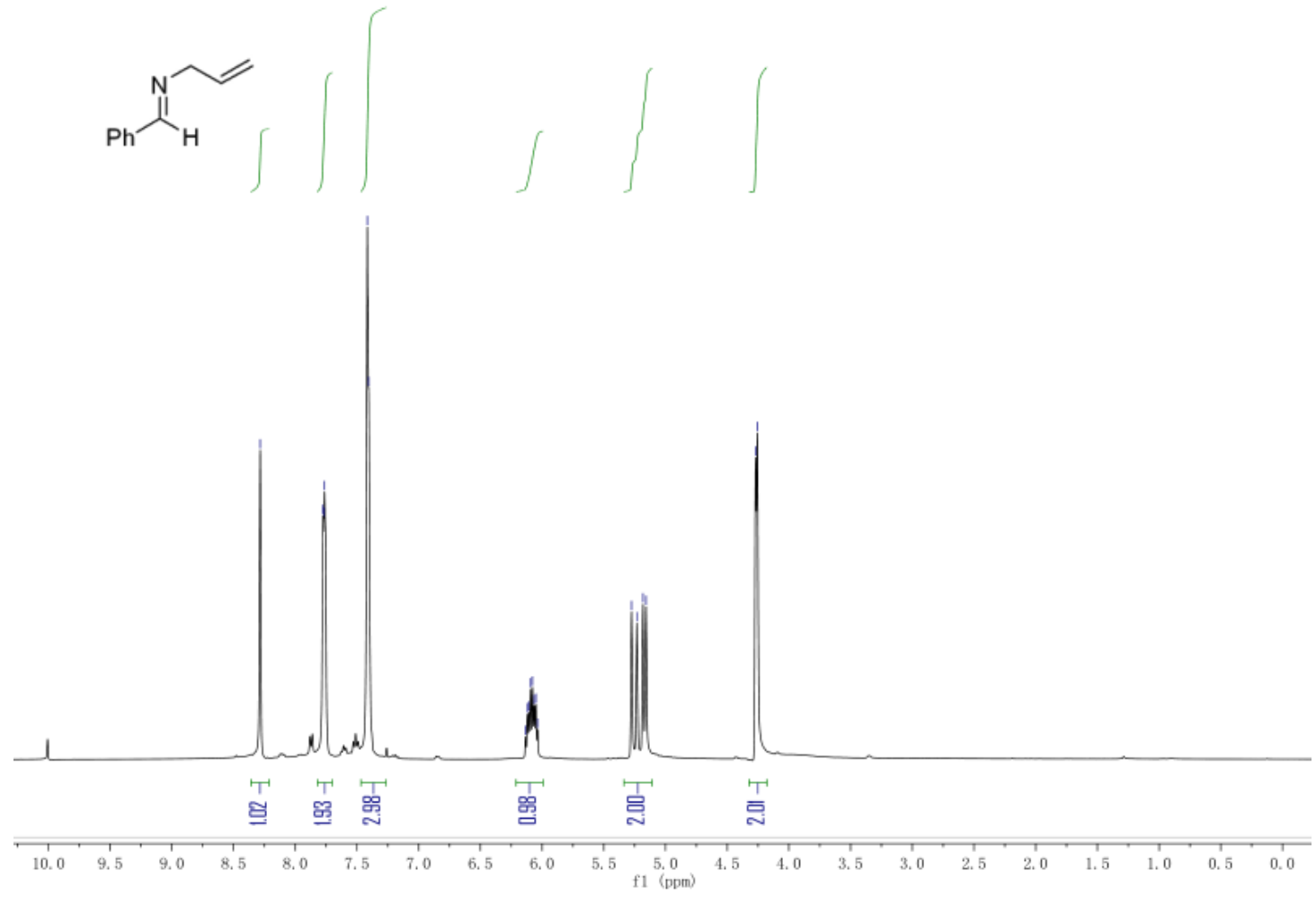


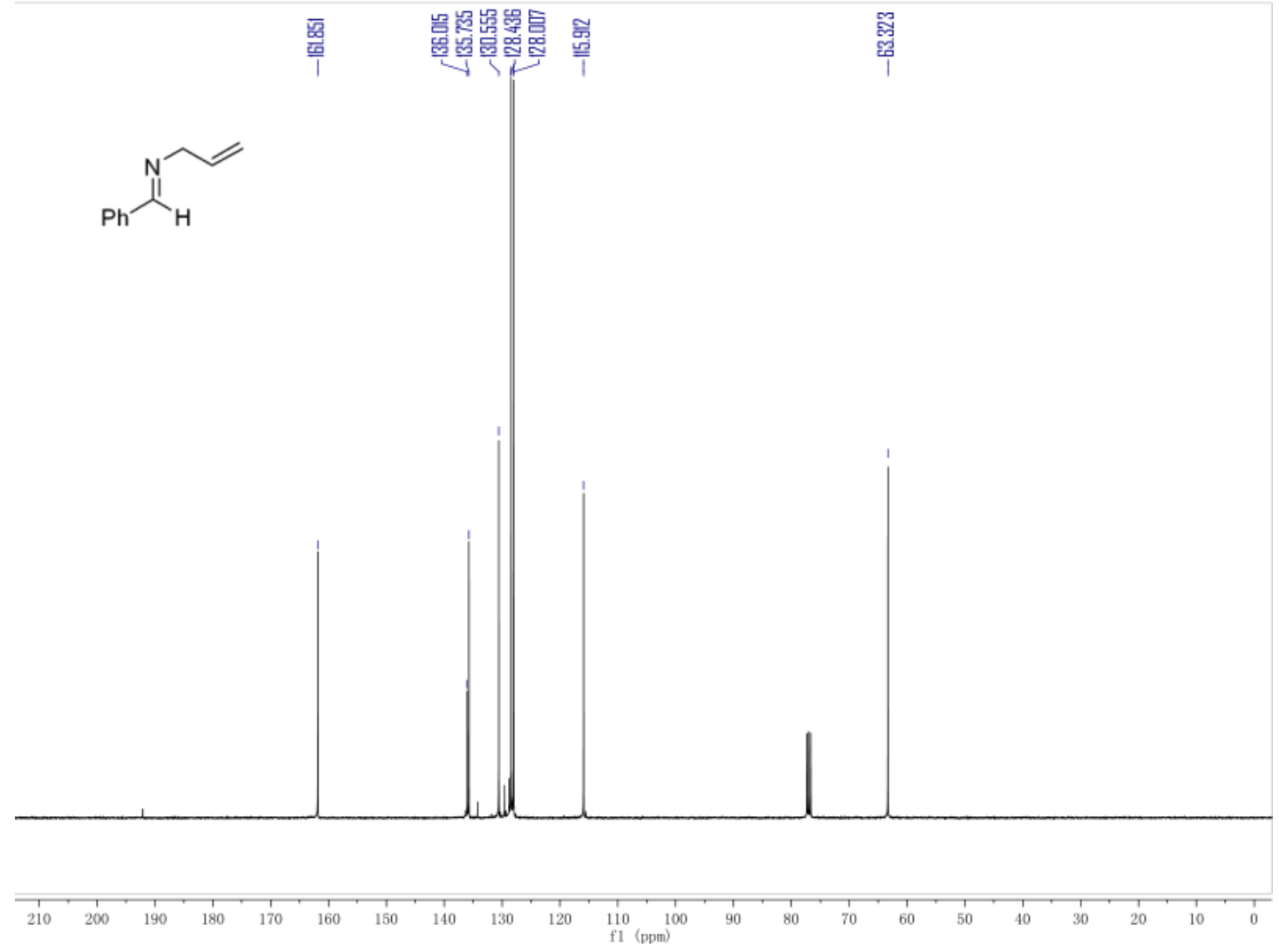

